NBSIR 88-3096

\title{
MEASUREMENT OF ADAPTER LOSS, MISMATCH, AND EFFICIENCY USING THE DUAL SIX-PORT
}

George J. Counas Bill C. Yates

National Bureau of Standards U.S. Department of Commerce Boulder, Colorado 80303-3328

July 1988

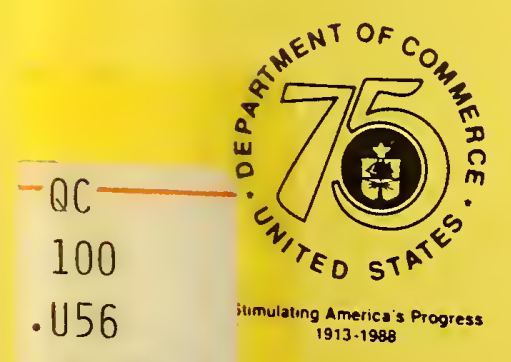





\section{MEASUREMENT OF ADAPTER LOSS, MISMATCH, AND EFFICIENCY USING THE DUAL SIX-PORT}

\section{George J. Counas Bill C. Yates}

Electromagnetic Fields Division Center for Electronics and Electrical Engineering National Engineering Laboratory National Bureau of Standards

Boulder, Colorado 80303-3328

July 1988

Supported in part by

Newark Air Force Station, Ohio 43057

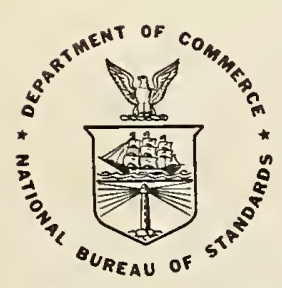

U.S. DEPARTMENT OF COMMERCE, C. William Verity, Secretary

NATIONAL BUREAU OF STANDARDS, Ernest Ambler, Director 

CONTENTS

\section{Page}

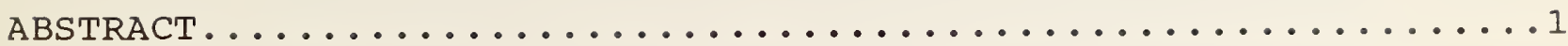

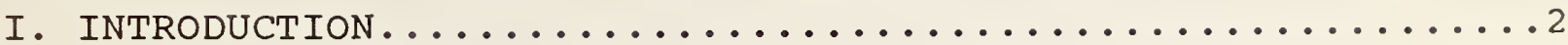

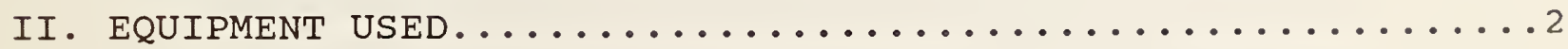

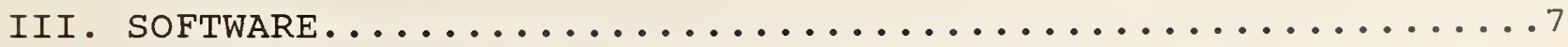

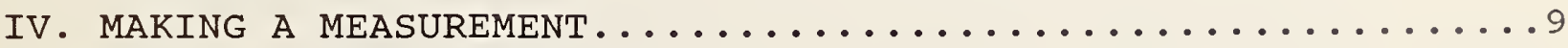

A. GENERAL OVERVIEW OF THE MEASUREMENT PROCESS..........9

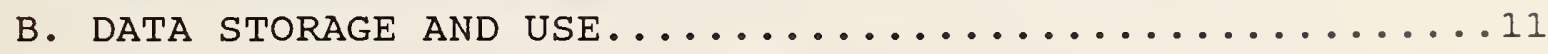

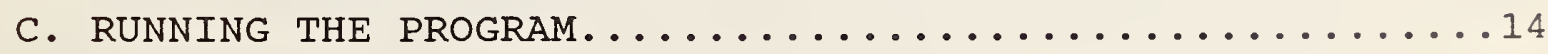

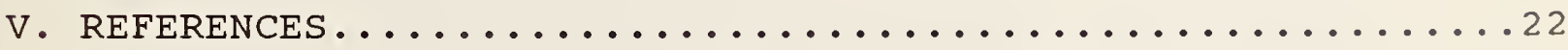

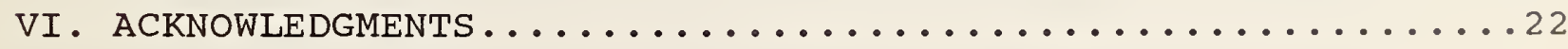

APPENDIX I LISTING OF ADAPTER PARAMETER CALCULATING

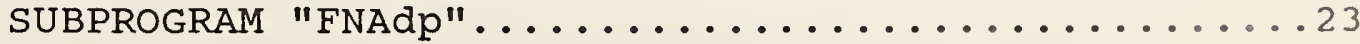

APPENDIX II LISTING OF ADAPTER PARAMETER CALCULATING SUBPROGRAM "Gamma".................... 40

APPENDIX III COMPLETE LISTING OF MEASUREMENT PROGRAM

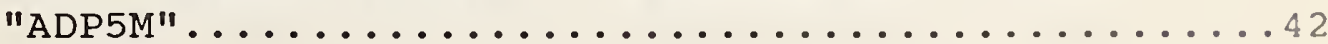




\section{LIST OF ILLUSTRATIONS AND TABLES}

FIGURE 1 SIMPLIFIED BLOCK DIAGRAM X-BAND FRONT END AND

PAGE

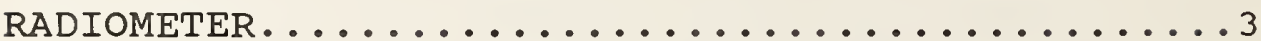

FIGURE 2 MEASUREMENT SETUP USING WAVEGUIDE TO COAX ADPT...5 FIGURE 3 MEASUREMENT SETUP FOR WAVEGUIDE TO COAX ADAPTER..6 FIGURE 4 FUNCTIONAL FLOW CHART FOR ADAPTER MEASUREMENT

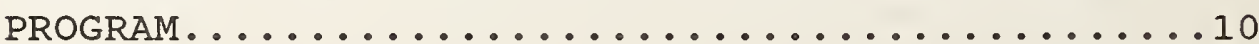

FIGURE 5 SAMPLE DATA LOG FOR USE WITH ADAPTER PROGRAM....12 FIGURE 6 SAMPLE PRINTOUT OF ADAPTER PARAMETERS SIX-

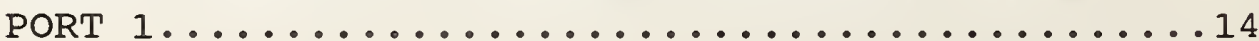

TABLE 1 IDEAL LENGTH FOR AIRLINES TO CALIBRATE DUAL SIXPORT WITH WAVEGUIDE INSERTION POINT.......17

TABLE 2 CONTENTS OF S MATRIX USED TO STORE ADAPTER

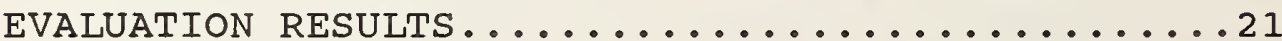


MEASUREMENT OF ADAPTER LOSS, MISMATCH, AND EFFICIENCY USING THE DUAL SIX-PORT

George J. Counas and Bill C. Yates

National Bureau of Standards

Boulder, Colorado 80303-3328

A noise measurement system is being developed for the U. S. Air force which uses coaxial cryogenic and ambient noise temperature standards to determine the noise temperature of the device under test. When the device under test has a different connector than those on the noise standards, an adapter has to be used. Adapter loss and complex reflection coefficients must be compensated for or noise measurement accuracy is affected. A technique has been developed which uses a dual six-port measurment system to determine the mismatch, loss, and ultimately the efficiency of the adapter used. This enables correction of measurement results and allows measurements to be made with an adapter with no degradation of accuracy.

This report describes this method of evaluating adapters and provides instructions for its use.

Key words: adapter parameters, dual six-port measurement system, efficiency, loss, measurement technique, mismatch. 
A noise measurement system is being developed at the Boulder Laboratories of the National Bureau of Standards for use by the Metrology Engineering section at Newark Air Force station. This system has accented the need for a reliable method of evaluating adapter input and output reflection coefficient, loss, and efficiency. A technique has been developed and tested which uses the dual six-port measurement system to ascertain these parameters. This technique requires calibrating the dual sixport system without adapters and then calibrating it again with adapters attached to the two measurement ports. Reflection coefficients and losses of the adapters on six-ports 1 and 2 are then calculated using the calibration results. After one further measurement which involves connecting the device under test to the adapter and determining the input reflection coefficient of this device, the efficiency of the combination can be calculated. If the device under test and adapter combination are left intact after this determination, an accurate correction for the the adapter can be made. In the following discussion, the adapter evaluation technique using the dual six-port measurement system is explained in simple terms. A detailed mathematical analysis of this technique is beyond the scope of this report. The main purpose of this report is to explain its function and use.

II. EQUIPMENT USED

The radiometer under developement is a complex multiband system. For simplicity, only the $\mathrm{x}$-band front of this system is shown in Figure 1 which is a block diagram of this portion of the 


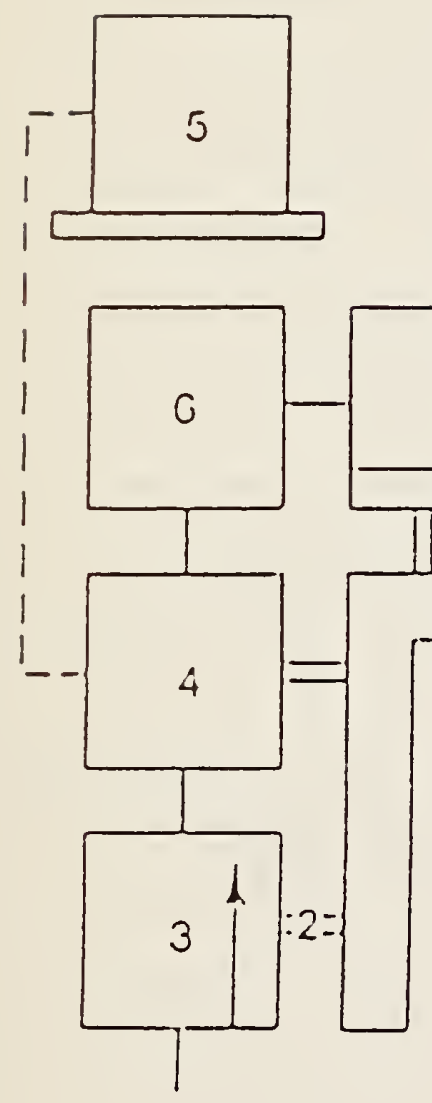

1. SUPPORT HOLDER

2. SUPPORT (5 PLACES)

3. COAXIAI ISOLATOR

4. FNPLIFIER

5. POWER SUPPIY

6. WAVEGUIDE TO COAY ADAPTER

7. ISOLATOR

8. HARMONIC FILTER

9. MIXER

10. IOCAL OSCILIATOR

11. AMPIIFIER

12. RFDIOMETER

SIMPLIFIED BLOCK DIAGRAM

$X$ BAND FRONT END AND RADIOMETER

FIGURE 1 
radiometer. The insertion point of this portion of the system is coaxial, but by using the evaluation technique described above, the loss, reflection coefficient, and efficiency of an X-band waveguide to coax adapter could be determined and the system then used to calibrate waveguide noise sources. See figure 2 for a typical noise measurement setup. Note that the two standards are coaxial and that the device under test is waveguide. Since the complex reflection coefficients and loss of the waveguide to coax adapter can be determined, the measurement can be made with very little degradation in accuracy. In a similar fashion, correction can be made for coaxial adapters used to connect a device under test with a different connector type than the one on the standards.

Figure 3 shows a typical setup for evaluating waveguide to coaxial adapters. The top portion of the figure shows the calibration system with no adapters while the bottom portion details the setup for measuring the loss and reflection coefficient of a waveguide to coax adapter. The two bends are used to eliminate any moding problems encountered when calibration standards are connected directly to the adapters. Short straight sections would do just as well but the bends were used to keep the insertion distance as short as possible and compensate for the right angle connections on the adapters.

The standards used to calibrate waveguide are the same as those used to calibrate coax except that in place of the open circuit calibration standards, quarter wave short circuits are used. These quarter wave short circuits need not be cut for the precise frequency of measurement. They should, however, be 


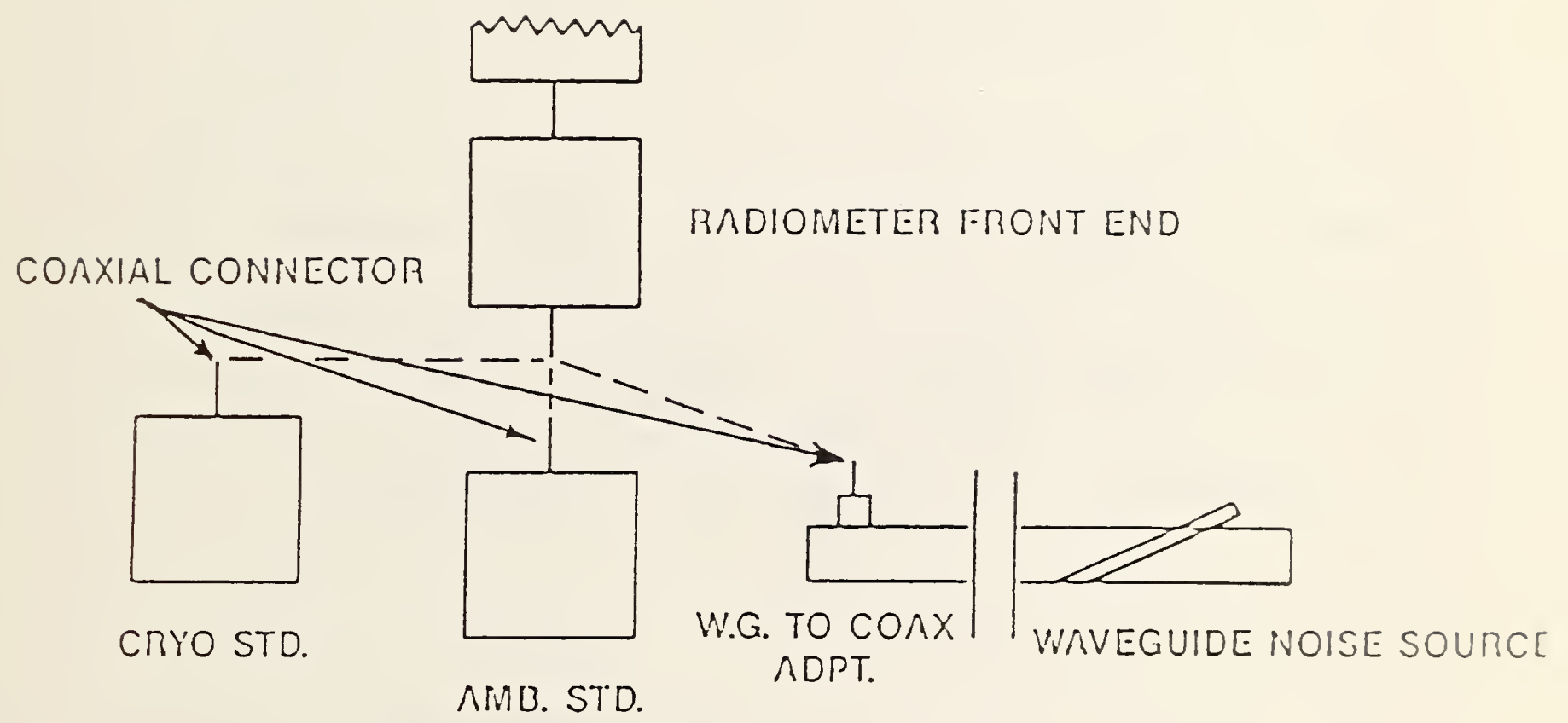

MEASUREMENT SETUP USING WAVEGUIDE TO COAX ADAPT.

FIGURE 2 

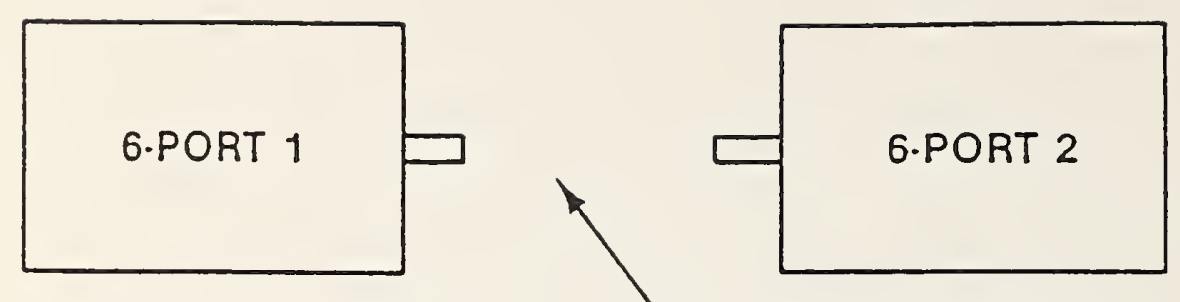

CALIBRATION POINT

USE COAXIAL STANDARDS

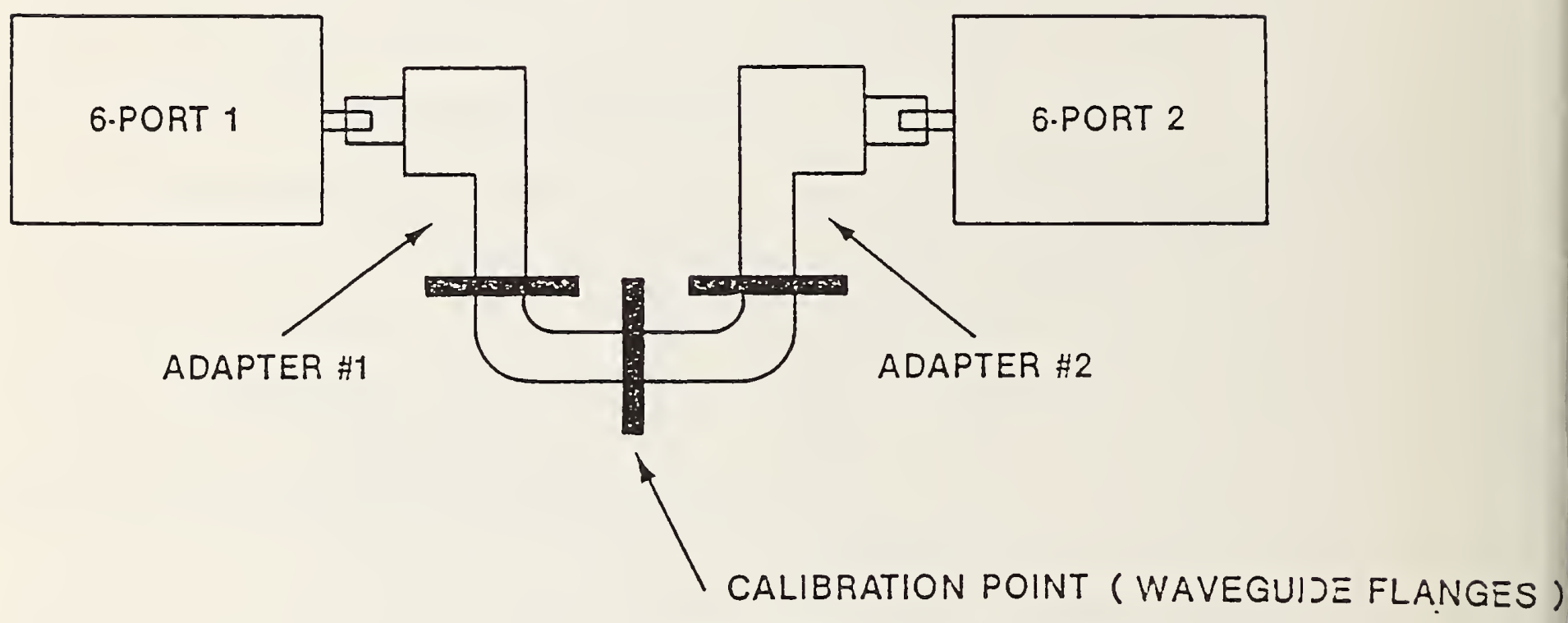

USE WAVEGUIDE STANDARDS

MEASUREMENT SETUP FOR WAVEGUIDE TO COAX ADAPTER

FIGURE 3 
bolted in place when in use. Also the calibration airline is replaced by a waveguide straight section which ideally is of the length to cause a 90 degree phase shift. Table 1 (page 17) shows the ideal length for airlines at frequencies in the WR-284, WR187, WR-137, and WR-90 waveguide sizes. The 90 degree column is the optimum length for a 90 degree airline. However, especially at higher frequencies, this length is shorter than practical. A workable airline can be achieved by adding to the 90 degree length, a length equivalent to 180 degrees until the desired length is obtained. The 180 degree column shows the length to add.

\section{SOFTWARE}

The software developed for adapter evaluation is modeled from a technique described by Glenn F. Engen in 1974 [1] and implemented by Ramon Jesch using an automatic network analyzer and power measurement system in 1977 [2].

The technique uses the following:

$$
\begin{aligned}
& S 11=\left(b^{\prime}-b\right) /\left(a-b^{\prime} c\right) \\
& S 22=\left(a^{\prime} c-a c^{\prime}\right) /\left(a-b^{\prime} c\right) \\
& S 12^{\wedge} 2=\left(a^{\prime}-b c^{\prime}\right) /\left(a-b^{\prime} c\right)+s 11 s 22
\end{aligned}
$$

where S11 and 522 are the complex reflection coefficients at each end of the adapter being measured and 512 is the transmission coefficient of the adapter from port 1 to port 2 . The complex parameters $\mathrm{a}, \mathrm{b}$, and $\mathrm{c}$ are obtained by calibrating the six-ports without the adapters and $\mathbf{a}^{\prime}, \mathrm{b}^{\prime}$, and c'are obtained by calibrating the six-ports with the adapters in place. Figure 3 is a block diagram showing the measurement of a waveguide to coax adapter pair. The loss and reflection coefficient of each 
adapter is obtained. $a^{\prime}, b^{\prime}$, and $c^{\prime}$ are not independent of $a, b$, and c. Some variables are obtained and stored during the calibration without the adapters and used to compute $\mathrm{a}^{\prime}, \mathrm{b}^{\prime}$, and c' with the adapters. These variables are computed in the "Iparab" and "Fparab" subprograms and are designated Zeta, Rho, W1, Re(W2), and IM(W2) in lines 5293 through 5298 of the adapter measurement program. The adapter measurement program is a modified version of the calibration program "CALBR". The modifications are mainly changes in program flow and include the addition of Subprograms "Gamma" and "FNAdpl1". These subprograms provide for the calculation of scattering coefficients and efficiency of the adapters on the two six-ports by using the following in addition to (1), (2), and (3):

Alpha $120=\operatorname{S} 12 m^{\wedge} 2 /\left(1-\operatorname{S} 11 m^{\wedge} 2\right)$

Alpha $210=S 21 \mathrm{~m}^{\wedge} 2 /\left(1-S 22 \mathrm{~m}^{\wedge} 2\right)$

where sllm, S22m, S12m, and S21m are scattering coefficient magnitudes for the adapter determined by using (1), (2), and (3). Alpha 120 and Alpha 210 are loss coefficients for the adapter from port 1 to 2 and port 2 to 1 respectively. Input power times the loss coefficient equals the output power.

$$
\mathrm{Gl}=\mathrm{S} 11+(\mathrm{S} 12 * \mathrm{~S} 21 * \mathrm{GX}) /(1-\mathrm{S} 22 * \mathrm{GX})
$$

where Gl is the calculated complex reflection coefficient of the device under test and adapter combination, and $\mathrm{Gx}$ is the complex input reflection coefficient of the device under test as measured from the adapter.

$$
\text { Alpha12 } \left.=\left(1-G^{\wedge} m^{\wedge} 2\right) * \operatorname{S12} m^{\wedge} 2\right) /\left(\left(1-G 1 m^{\wedge} 2\right) *[1-S 22 G x]^{\wedge} 2\right)
$$

where Alpha 12 represents the efficiency of the device under test and adapter combination and Gxm, S12m, Glm, and [1-S22Gx] are 
magnitudes of previously determined quantities.

The quantity Alpha 12 is the correction factor for the adapter. Divide the measured noise temperature by this factor if the adapter is on the device under test; Multiply by this factor if the adapter is on the standard.

For convenience, listings of these subprograms are included in APPENDIX I of this report. The current version of the complete adapter measurement program has been named "ADPM5"

IV. MAKING A MEASUREMENT

A. GENERAL OVERVIEW OF THE MEASUREMENT PROCESS

The operator will find little difference between using this program and the software (PROGRAM "CALBR") furnished with the six-port measurement system. Program flow has been modified so that two calibrations are performed instead of one and a measurement of the device under test attached to the selected adapter is made. An additional store and print segment has been added to print adapter parameters and save them for use later.

A flow diagram describing the operation of program "ADPM5" is shown in Figure 4. As can be seen in the figure, calibration frequencies and other calibration parameters are input. Then, calibration of the dual six-port measurement system is performed first without the adapters under test, and then with the adapters. Finally, after the two calibrations, the device under test is attached to the desired adapter and measured. The power data obtained from the three operations is processed and the losses, reflection coefficients, and efficiencies of the adapters on both six ports are calculated and stored.

A log of data collected from calibration of the six-ports 


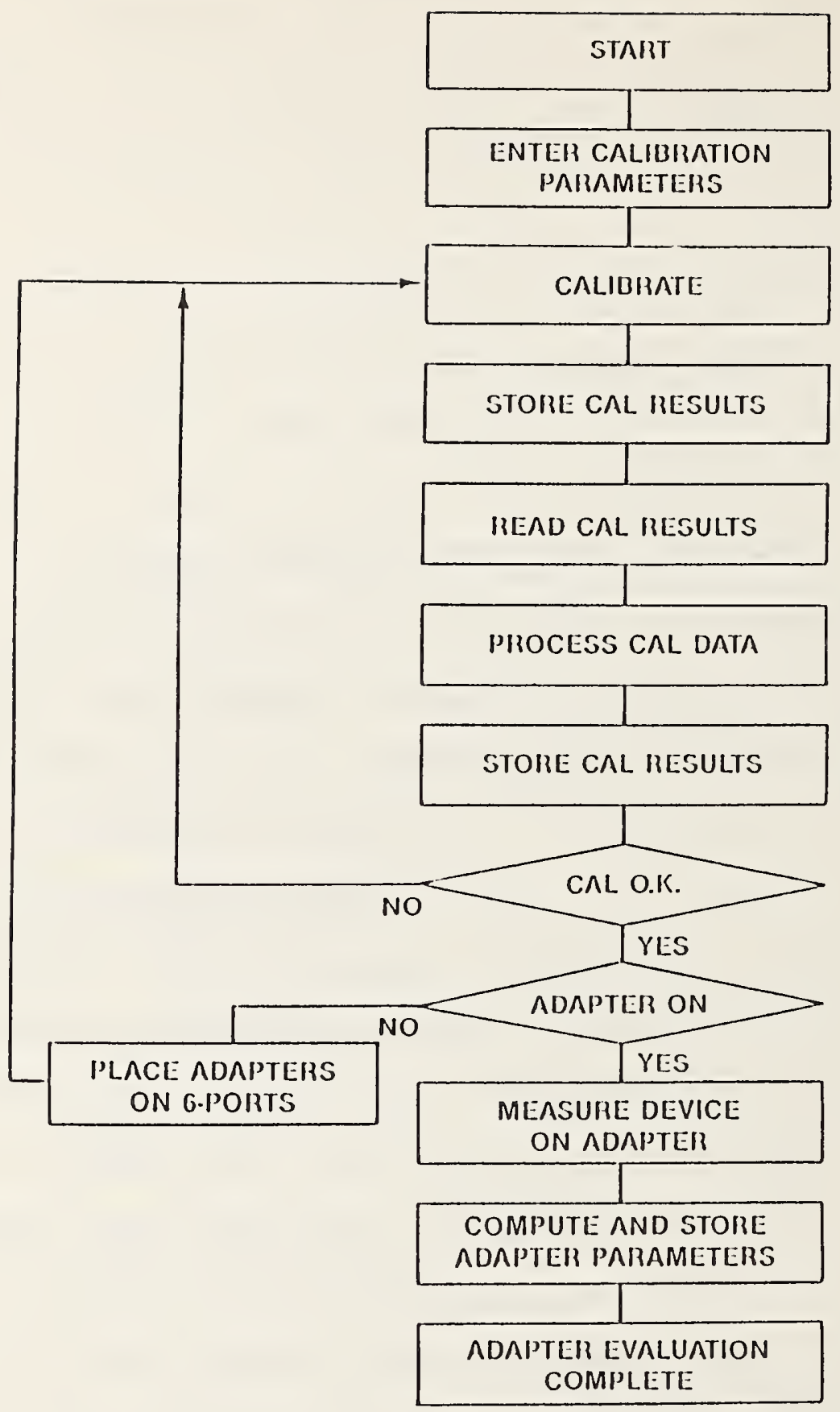

FUNCTIONAL FLOW CIIAIT

FOII

ADAPTER MEASUREMENT

PHOGIAM

FIGURE 4 
hould be maintained. Figure 5 is a sample data log showing the ata stored in one adapter evaluation. The program storage is rranged so that the files shown are overwritten if a new valuation is made. Permanent storage of calibration results is rovided in user named files if this is desired. The prompt: "DO OU WANT TO SAVE SYSTEM PARAMETER DATA ON MASS STORAGE--Y/N" is isplayed at the end of each calibration sequence. A yes answer s followed by prompts asking for file names desired and for a lisc to be placed in the correct disc drive. A no answer exits :he routine and program execution continues.

3. DATA STORAGE AND USE

The first functions performed during program execution are alibrations of the six-ports with and without adapter. The cesults of the calibrations are stored in the power matrix files lesignated with a prefix of PWRH and PWRD. A number is added to this prefix to provide identification for the file. PWRHI zontains header information and describes the data file PWRDI which contains the power data from the first calibration (without the adapters). After calibration, the power data is processed and file CRSTl is created. This file contains the results of the first calibration. The second calibration (with adapters) is now made and files PWRH2, PWRD2, and CRST2 are created and filled. After the calibrations, a device under test (usually a noise source with one output port is connected to the appropriate adapter and measured. Power data from this measurement is stored in file 1PRD3 which is described by header file 1PRH3. After the measurement of the device under test, subprogram "Gamma" automatically accesses the correct files and computes, displays, 


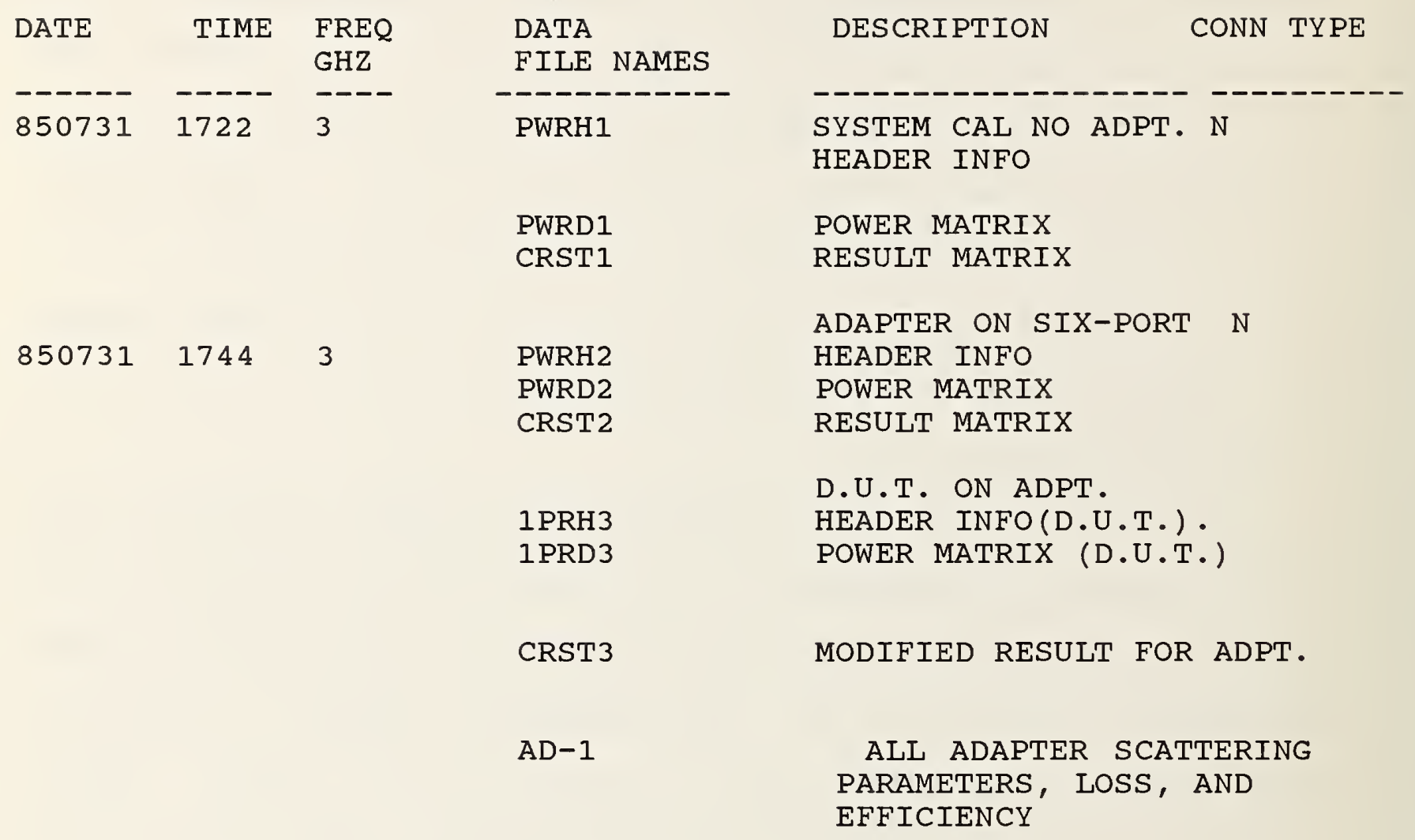

SAMPLE DATA LOG FOR USE WITH ADAPTER PROGRAM FIGURE 5 
and stores the reflection coefficients for the device under test--adapter combination. subprogram "FNAdpt" then automatically retrieves data and calculates scattering coefficients, loss, and efficiency of the adapter. This data is stored in file $\mathrm{AD}-1$ in the S matrix. A list of the contents of this matrix is found in Table 2 which is intended to be an aid in data retrieval. This data will be used to correct the noise temperature of the device under test for adapter error.

Figure 6 is a sample printout from the final phase of the program and details the loss and reflection coefficient of the adapters on both six-ports. The adapters used here were Type $N$ male to female adapters.

C. RUNNING THE PROGRAM

Load "ADPM5" and press run.

As the program is run, prompts displayed on the screen lead the operator through required operations step by step.

1. The first prompt asks for the input option.

1) Start, stop, and step list

2) Type in list

5) Exit

Answer with the appropriate response (usually 1)

2. The second prompt asks:

Input six-port option

1) $<=2 \mathrm{GHZ}$ 6-port

2) >=2ghz 6-port

Answer with the appropriate response.

3. The third prompt asks for frequency input as specified by options 1 or 2 . 
FILES PROCESSED=CRST5 AND CRST6

FREQUENCY $=7 \mathrm{GHZ}$

ADAPTOR LOSS AT 6PORT NUMBER 1

SYSTEM CONSTANTS

$\mathrm{A} 8, \mathrm{~A} 9, \mathrm{~B} 8, \mathrm{~B} 9, \mathrm{C} 8, \mathrm{C} 9$

$\mathrm{A} 1, \mathrm{~A} 2, \mathrm{B1}, \mathrm{B} 2, \mathrm{C1}, \mathrm{C} 2 \quad .36931454192$

.20671621467

.525670491353

$-1.758488244 \mathrm{E}-02-.250158691953$

$7.2367883 E-02$

ADAPTER CONSTANTS

$\mathrm{A} 8, \mathrm{~A} 9, \mathrm{~B} 8, \mathrm{~B} 9, \mathrm{C} 8, \mathrm{C} 9$

$-6.40256 \mathrm{E}-02$

$-.418296616$

.53012308

$4.721305 \mathrm{E}-02$

$-3.36437 \mathrm{E}-02$

$-.259555044$

$\mathrm{A} 1, \mathrm{~A} 2, \mathrm{~B} 1, \mathrm{~B} 2, \mathrm{C1}, \mathrm{C} 2-6.402565 \mathrm{E}-02$

$-4.721805 \mathrm{E}-02$

$-3.364376 \mathrm{E}-02$

$-.41825661$

$-.25955504$

.53012307

s12m,s12a,s12r,s12a

.98546034012

$-68.400123$

.36277018

$-.9162586$

IMPEDANCE DATA FOR ADAPTER PM SIX-PORT \#1

S11 MAGNITUDE

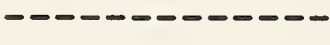

.1030

S22 MAGNITUDE

- - - - - - - - -

.1017
SII ANGLE

$-119.84$

S2 2 ANGLE

$--------$

162.6897
S11 REAL

-ーーーーーーー

$-.0513$

S22 REAL

--------

$-.0971$
S11 IMAGINARY

$-.0893$

S22 IMAGINARY

.0303

LOSS DATA FOR ADAPTER ON SIX-PORT 1

$\begin{array}{lc}\text { ALPHA } 12 & \text { LOSS DB } \\ -0---0 & -0 .-- \\ .9815 & .0809\end{array}$

ALPHA 21

LOSS DB

.9813

.0820

SAMPLE PRINTOUT OF ADAPTER PARAMETERS SIX-PORT 1

FIGURE 6 
4. After the frequecies desired are entered, the fourth prompt asks :

Enter options for next operation

1) Input frequency

2) Determine system parameters without power standard

3) Measure 1-port on adapter

4) Evaluate Adapters

5) Exit

A response of 1 returns to the frequency input section of the program. A response of 2 is the normal input for a calibration sequence. A response of 3 is given only after both calibration have been performed. A response of 4 is given after both calibrations and the measurement of the one port device.

(This segment of the program is returned to after each task is accomplished and the program proceeds in response to the answers to the prompts).

5. The fifth prompt:

Turn on amplifiers Press continue when ready

6. The sixth prompt:

Enter connector type option

1) $14 \mathrm{~mm}, 7 \mathrm{~mm}$, or waveguide

2) Type $\mathrm{N}$ male adapter on 6-port 1

3) Type $\mathrm{N}$ Female adapter on 6-port 1

4) Other, not available at present

Enter the desired response.

7. The seventh prompt asks for connection of shorts, opens, a measurement with system ports connected, a 2-port, and 
airline with measurements being made on each device

and the powers stored at the end of the sequence in a PWRDI or PWRD2 file. After the first calibration, prompt 4 above is again displayed, and the program execution is repeated from that point until the two calibrations are completed.

When the second calibration is completed, prompt 4 is again displayed. This time answer with 3. This causes the program to access the measurement routines. Place the device under test on the appropriate adapter and continue program execution.

After the device under test is measured, prompt 4 is again displayed. This time answer the prompt with 4. This causes the program to automatically process the stored data and print and store the adapter evaluation data. When this has been accomplished, prompt 4 is again displayed. This time the correct response is 5. The beginning of the program is now accessed and measurements are complete. Press stop if no further measurements are desired.

The accuracy of the results obtained by this technique depends on a variety of factors. One way to evaluate systematic error is to perform the above tasks without inserting an adaptor. The varience of efficiency from unity is a measure of this error. This varience is usually less than .002. 
TABLE 1

IDEAL LENGTH FOR AIRLINES TO CALIBRATE DUAL SIXPORT WITH WAVEGUIDE INSERTION POINT

WR-284 WAVEGUIDE

FREQUENCY GHZ

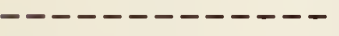

2.6

2.7

2.8

2.9

3.0

3.1

3.2

3.3

3.4

3.5

3.6

3.7

3.8

3.9

3.95

\section{GUIDE WAVELENGTH INCHES}

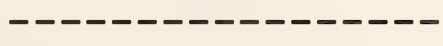

7.55

6.85

6.29

5.83

5.45

5.13

4.85

4.60

4.39

4.19

4.02

3.85

3.71

3.57

3.51
IDEAL AIRLINE INCHES

90 DEG 180 DEG

-----------------

$1.89 \quad 3.78$

1.713 .43

$1.57 \quad 3.15$

$1.46 \quad 2.92$

$1.36 \quad 2.73$

$1.28 \quad 2.57$

$1.21 \quad 2.43$

1.152 .30

$1.10 \quad 2.20$

$1.05 \quad 2.10$

$1.00 \quad 2.01$

0.961 .93

0.931 .86

$0.89 \quad 1.79$

$0.88 \quad 1.76$

WR187 WAVEGUIDE

FREQUENCY GHZ

4.0

4. 1

4.2

4.3

$4 \cdot 4$

4.5

4.6

4.7

4.8

4.9
GUIDE WAVELENGTH INCHES

IDEAL AIRLINE INCHES

90 DEG 180 DEG

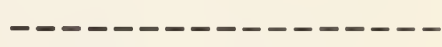

4.79

1.20

2.40

4.50

1.13

2.25

4.25

1.06

2.13

4.04

1.01

2.02

3.84

0.96

1.92

3.68

0.92

1.84

3.52

0.88

1.76

3.39

0.85

1.70

3.26

0.82

1.63

3.15

1. 58 
TABLE 1 (CONTINUED)

WR187 WAVEGUIDE (CONT)

FREQUENCY GHZ GUIDE WAVELENGTH
INCHES
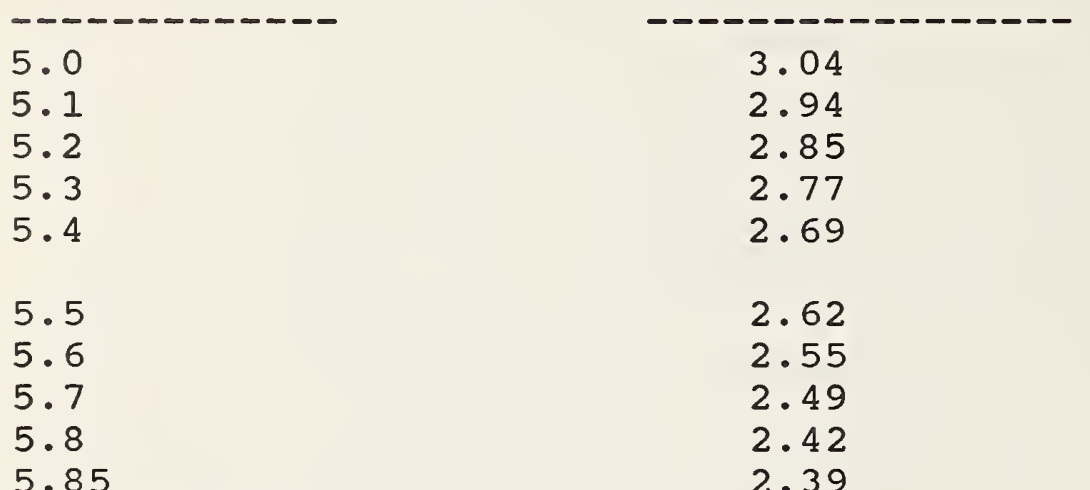

2.62

2.55

2.49

2.42

2.39

\section{GUIDE WAVELENGTH \\ INCHES}

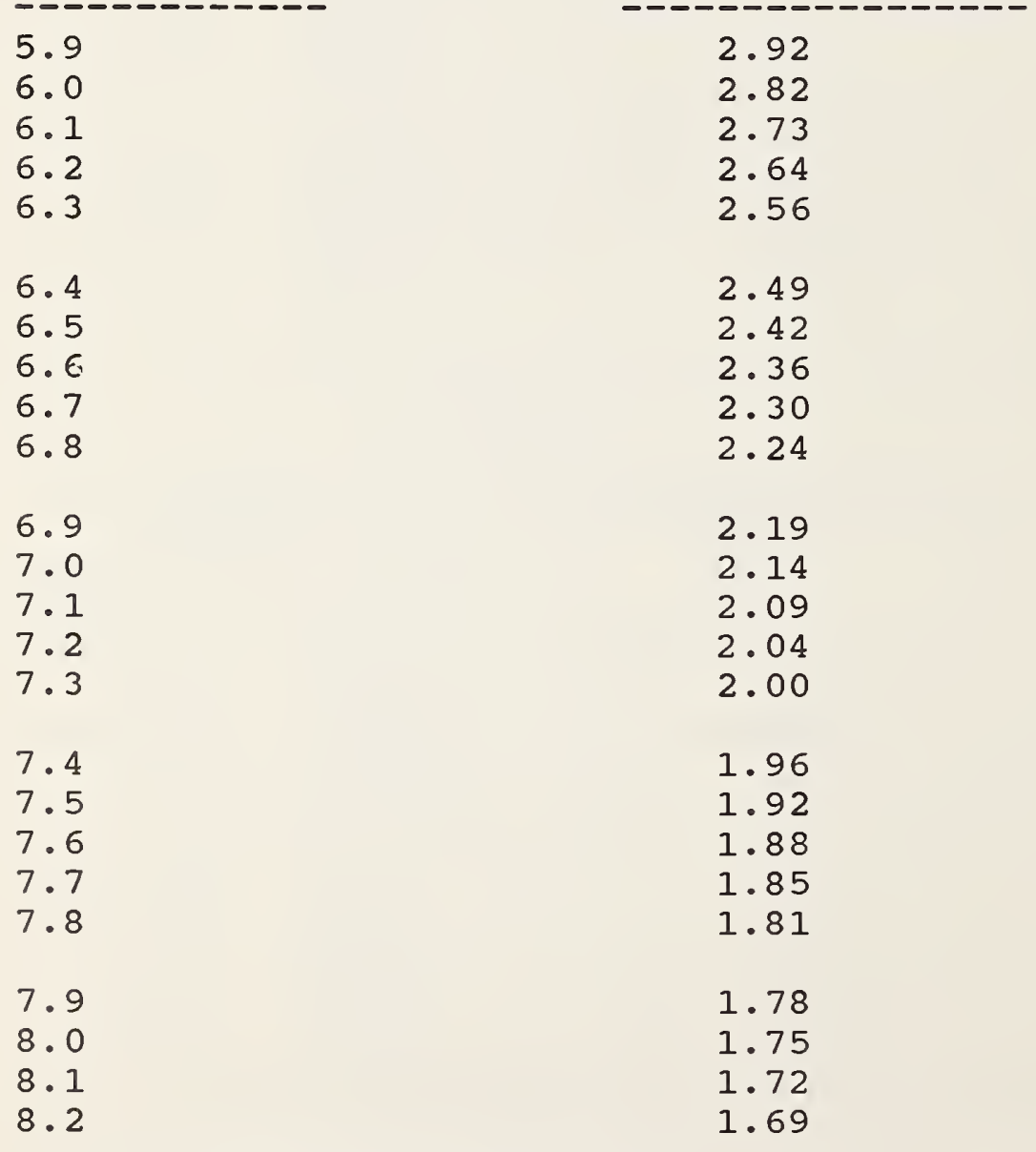

FREQUENCY GHZ

WR137 WAVEGUIDE

IDEAL AIRLINE INCHES

90 DEG 180 DEG

$\begin{array}{ll}0.76 & 1.52 \\ 0.74 & 1.47 \\ 0.71 & 1.43 \\ 0.69 & 1.39 \\ 0.67 & 1.35\end{array}$

$0.65 \quad 1.31$

$0.64 \quad 1.28$

$0.62 \quad 1.25$

$0.61 \quad 1.21$

$0.60 \quad 1.20$ 
TABLE 1 (CONTINUED)

WR90 WAVEGUIDE

FREQUENCY GHZ

8.2

$8 \cdot 3$

8.4

8.5

$8 \cdot 6$

8.7

8.8

8.9

9.0

9.1

9.2

9.3

9.4

9.5

9.6

9.7

9.8

9.9

10.0

10.1

10.2

10.3

10.4

10.5

10.6

10.7

10.8

10.9

11.0

11.1

11.2

11.3

11.4

11.5

11.6

11.7

11.8
GUIDE WAVELENGTH

INCHES

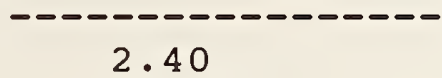

2.32

2.25

2.18

2.12

2.06

2.01

1.96

1.91

1.87

1.83

1.79

1.75

1.72

1.68

1.65

1.62

1.59

1.56

1.54

1.51

1.49

1.46

1.44

1.42

1.40

1.38

1.36

1.34

1.32

1.30

1.28

1.27

1.25

1.23

1.22

1.20
IDEAL AIRLINE INCHES

90 DEG 180 DEG

$\begin{array}{ll}0.60 & 1.20 \\ 0.58 & 1.16 \\ 0.56 & 1.12 \\ 0.55 & 1.09 \\ 0.53 & 1.06\end{array}$

$0.52 \quad 1.03$

$0.50 \quad 1.01$

$0.49 \quad 0.98$

$0.48 \quad 0.96$

$0.47 \quad 0.94$

$\begin{array}{ll}0.46 & 0.91\end{array}$

$0.45 \quad 0.89$

0.440 .88

$0.43 \quad 0.86$

0.420 .84

$0.41 \quad 0.83$

$0.41 \quad 0.81$

$0.40 \quad 0.80$

$0.39 \quad 0.78$

$0.38 \quad 0.77$

$0.38 \quad 0.76$

$0.37 \quad 0.74$

$0.37 \quad 0.73$

$\begin{array}{lll}0.36 & 0.72\end{array}$

$0.35 \quad 0.71$

$0.35 \quad 0.70$

$0.34 \quad 0.69$

0.340 .68

$0.33 \quad 0.67$

$0.33 \quad 0.66$

$0.32 \quad 0.65$

$0.32 \quad 0.64$

0.320 .63

$\begin{array}{lll}0.31 & 0.62\end{array}$

$\begin{array}{lll}0.31 & 0.62\end{array}$

$0.30 \quad 0.61$

$0.30 \quad 0.60$ 
TABLE 1 (CONTINUED)

\section{WR9O WAVEGUIDE (CONT)}

\section{FREQUENCY GHZ}

11.9

12.0

12.1

12.2

12.3

12.4
GUIDE WAVELENGTH INCHES

1.19

1.17

1.16

1.15

1.13

1.12
IDEAL AIRLINE INCHES

90 DEG 180 DEG

$0.30 \quad 0.59$

0.290 .59

0.290 .58

$0.29 \quad 0.57$

$0.28 \quad 0.57$

$0.28 \quad 0.56$ 
TABLE 2

CONTENTS OF S MATRIX USED TO STORE ADAPTER EVALUATION RESULTS

MATRIX ELEMENT

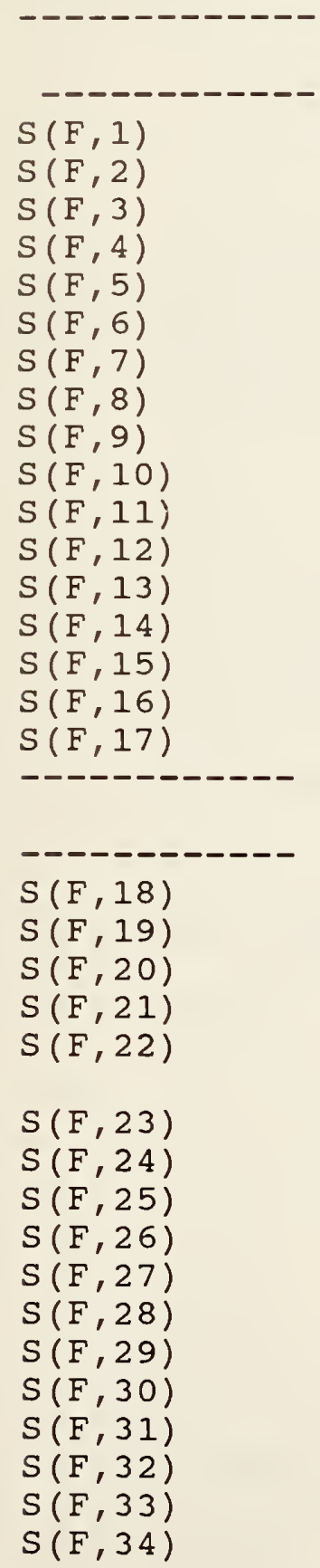

DESCRIPTION

ADAPTER ON SIX-PORT ONE

S11 MAGNITUDE

S11 PHASE

SII REAL PART

S11 IMAGINARY PART

ALPHA 12

S22 MAGNITUDE

S22 PHASE

S22 REAL PART

S22 IMAGINARY PART

ALPHA 21

S12 MAGNITUDE

S21 MAGNITUDE

REFL. COEFF. OF D.U.T. AND ADAPT.

REFL. COEFF. ANGLE OF D.U.T. AND ADPT.

REAL PART OF $S(F, 14)$

IMAGINARY PART OF $S(F, 14)$

EFFICIENCY OF ADAPTER

ADAPTER ON SIX-PORT TWO

SII MAGNITUDE

S11 PHASE

S11 REAL PART

S11 IMAGINARY PART

ALPHA 12

S22 MAGNITUDE

S2 2 PHASE

S22 REAL PART

S22 IMAGINARY PART

ALPHA 21

S12 MAGNITUDE

S21 MAGNITUDE

REFL. COEF. OF D.U.T. AND ADPT.

REFL. COEF. PHASE OF D.U.T. AND ADPT.

REAL PART OF $S(F, 30)$

IMAGINARY PART OF $S(F, 30)$

EFFICIENCY OF ADAPTER

NOTE: THE $F$ IN THE MATRIX IDENTIFIER REFERS TO THE FREQUENCY NUMBER. 
V. REFERENCES

[1] Engen, Glenn F. Calibration Techniques for Automatic Network Analyzers with Application to Adapter Evaluation; IEEE Transactions on Microwave Theory and Techniques; Vol MT-22, No. 12; December 1974.

[2] Jesch Ramon C. Evaluation of Low-Loss/Low Reflection Two-Port Devices or Adapters by Automated Measurement Techniques; NBSIR 78-870; 1977 .

\section{ACKNOWLEDGMENTS}

The authors wish to acknowledge the technical assistance and support by Bill Daywitt and John Juroshek and the financial support rendered by the Metrology Engineering section of Newark Air Force station. 
LISTING OF ADAPTER PARAMETER CALCULATING SUBPROGRAM "FNAdP"

6470 DEF FNAdp (Nfreqs, Repeats,M1, P1, M2, P2, CA\$)

6480 !Calc Parameters for Adapter from 6-Port Measurements 850805

6490 OPTION BASE 1

6500 Msus $\$=": F 8,1 "$

6510 DIM Result(Nfreqs, 60), Freqs (Nfreqs), Id_content $\$[80]$, S (Nfreqs, 34)

$6521 \mathrm{~K}=0$

6570 ! IINPUT "ENTER FILE NAME FOR DATA WITHOUT ADAPTER";NI\$

6580 FIXED 0

6590 ! LINPUT"ENTER FILE NAME FOR DATA WITH ADAPTER";N2\$

$6620 \mathrm{~N} 1 \$=" C R S T 1 "$

$6630 \mathrm{~N} 2 \$=" \mathrm{CRST} 3$

6640 CALL Redhed(N1\$,Msus\$, Date\$,Time\$,Typ\$,Nfregs, Freqs(*), Repeats, Sp, std_mnt, Id_contents\$,Cnnct\$)

6650 MASS STORAGE IS ":F8, I"

6660 PRINTER IS 16

6670 PRINT "START OF FNADPT"

6680 Nfreg=N=Nfregs

6690 PRINT

6700 PRINT

$6710 \$$ Sport $=1$

6720 PRINTER MS 0

6721 PRINT

6724 PRINT "ADAPTER EVALUATION"

6725 PRINT"-_-_-_-_- 
6727 PRINT

6730 PRINT "FILES PROCESSED=";N1\$; " AND " i N2\$

6740 PRINT

$6750 \quad N 1=I N T(N 1)$

6760 N2=INT $($ N2)

6770 PRINTER IS 16

6780 FOR $\mathrm{P}=1$ to Nfreqs

6790 FOR Prt=1 to 2

6800 PRINTER IS 16

6810 ON Prt GoTO 6820,6850

6820 CALL Redwrt(N2\$,":F8, 1"," ",Result(*), Nfreqs)

$6830 \quad$ Nereq $=1$

6840 GOTO 6870

6850 CALL Redwrt(N2\$,":F8, 1"," ".Result(*),Nfreqs)

6860 NORMAL

6870 ON Sport GOTO 6880,6900

$6880 \mathrm{MC}=0$

6890 GOTO 6910

$6900 \mathrm{MC}=12$

6910 A8 $=$ Result $(P, 6+M C)$

6920 A9=Result $(P, 7+M C)$

$6930 \mathrm{~B} 8=\operatorname{Result}(\mathrm{P}, 8+\mathrm{MC})$

6940 B9=Result $(P, 9+M C)$

$6950 \mathrm{C} 8=$ Result $(P, 10+M C)$

$6960 \mathrm{C} 9=$ Result $(\mathrm{P}, 11+\mathrm{MC})$

6980 GOTO 6990

$6990 \mathrm{~A} 11$ (Prt) $=\mathrm{A} 8$

7000 A22 (Prt) $=$ A9 
$7010 \mathrm{~B} 11$ (Prt) $=\mathrm{B} 8$

7020 B22 (Prt) $=B 9$

$7030 \operatorname{cl} 1(\operatorname{Prt})=\mathrm{C} 8$

$7040 C 22($ Prt $)=C 9$

7050 ON Prt GOTO 7060,7140

7060 FIXED 4

7061 PRINTER IS 16

7070 PRINT "FREQUENCY = "iFreqs (P) :" GHZ"

7080 PRINT

7090 PRINT "ADAPTER LOSS AT 6PORT NUMBER ";sport

7100 PRINT

7110 PRINT "SYSTEM CONSTANTS"

7120 PRINT

7130 GO TO 7180

7140 PRINT

7150 PRINTER IS 16

7160 PRINT "ADAPTER CONSTANTS"

7170 PRINT

7180 FIXED 4

7190 PRINT "A8, A9, B8, B9, C8, C9"

7200 PRINT A8, A9, B8, B9, C8, C9

7210 PRINT

7220 PRINT "A1,A2,B1, B2,C1,C1" ; A11 (Prt), A22 (Prt), B11 (Prt), B22 (Prt), C11 (Prt), C22 (Prt)

7230 PRINT

7240 NEXT Prt

$7250 !$ 
$7260 ! S 11=\left(b^{\prime}-b\right) /\left(a-b^{\prime} c\right)$

$7270 !$

7280 !

$7290 \quad \mathrm{Z} 1=\mathrm{B} 11$ (2)

$7300 \mathrm{Z2}=\mathrm{B} 22(2)$

$7310 \mathrm{Z3}=\mathrm{B} 11(1)$

$7320 \quad \mathrm{Z} 4=\mathrm{B} 22(1)$

7330 CALL Csua $(z 1, z 2, z 3, z 4, z 9, z 0)$

$7340 \mathrm{Nr}=\mathrm{Z9}$

$7350 \mathrm{Ni}=\mathrm{Z} 0$

$7360 \mathrm{Z3}=\operatorname{Cl1}(1)$

$7370 \quad \mathrm{Z} 4=\mathrm{C} 22(1)$

7380 CALL Cmua $(\mathrm{Z} 1, \mathrm{Z2}, \mathrm{Z3}, \mathrm{Z} 4, \mathrm{Z9}, \mathrm{z} 0)$

$7390 \quad \mathrm{Z} 3=\mathrm{Z} 9$

$7400 \quad \mathrm{Z} 4=\mathrm{Z} 0$

7410 Z1=A11 (1)

$7420 \mathrm{Z2}=\mathrm{A} 22(1)$

7430 CALL Csua $(\mathrm{z} 1, \mathrm{z} 2, \mathrm{z} 3, \mathrm{z} 4, \mathrm{z} 9, \mathrm{z} 0)$

$7440 \quad \mathrm{Z} 1=\mathrm{Nr}$

$7450 \quad \mathrm{Z} 2=\mathrm{Ni}$

$7460 \quad \mathrm{Z} 3=\mathrm{Z} 9$

$7470 \quad \mathrm{Z} 4=\mathrm{Z} 0$

7480 CALL Cdia(z1,Z2,Z3,Z4,Z9,Z0)

7490 S11r=Z9

7500 S $11 i=Z 0$

$7510 \mathrm{X}=\mathrm{Z9}$

$7520 \quad \mathrm{Y}=\mathrm{ZO}$

7530 CALL CRPA $(X, Y, R, T)$ 


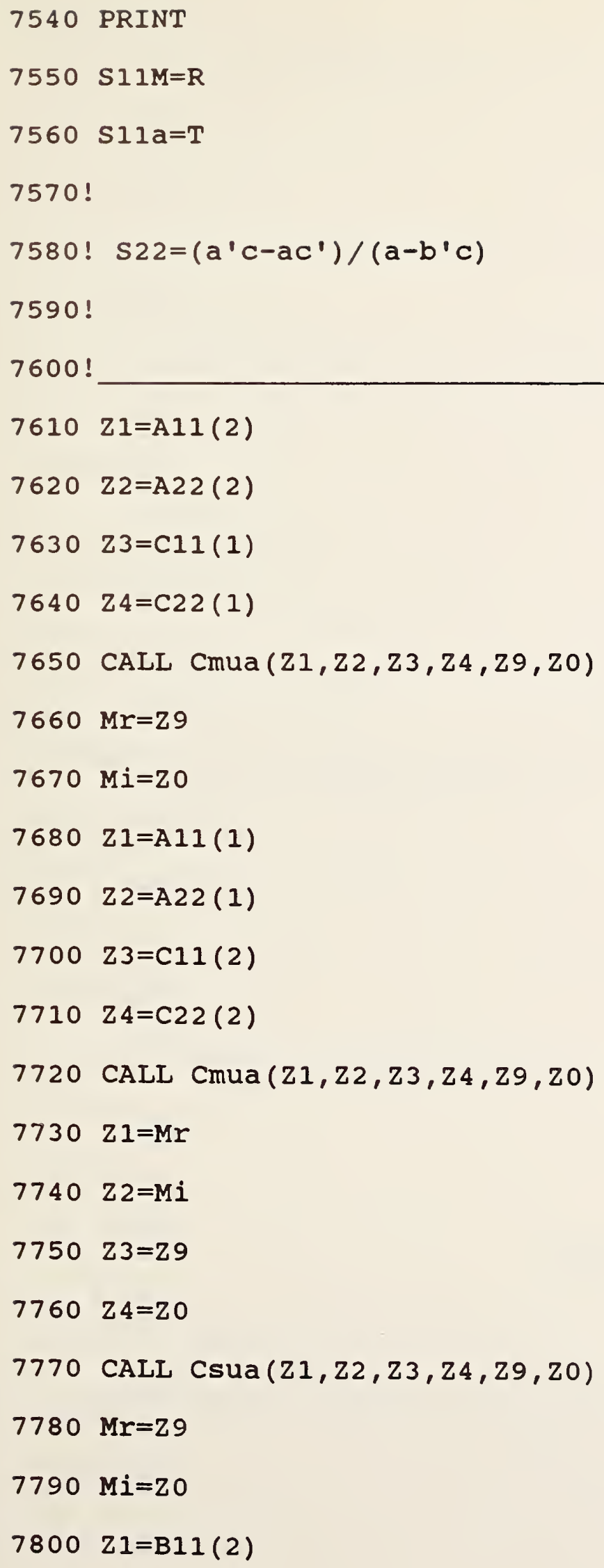


$7810 \quad \mathrm{Z} 2=\mathrm{B} 22(2)$

$7820 \quad 23=\operatorname{Cl1}(1)$

$7830 \quad 24=C 22(1)$

7840 CALL Cmua $(Z 1, Z 2, Z 3, Z 4, Z 9, Z 0)$

$7850 \quad \mathrm{Z} 3=\mathrm{Z} 9$

$7860 \quad \mathrm{Z} 4=\mathrm{Z} 0$

$7870 \quad \mathrm{Z} 1=\mathrm{A} 11$ (1)

$7880 \quad \mathrm{Z} 2=\mathrm{A} 22(1)$

7890 CALL Csua $(\mathrm{Z} 1, \mathrm{Z2}, \mathrm{Z3}, \mathrm{Z4}, \mathrm{Z9}, \mathrm{Z} 0)$

$7900 \quad \mathrm{Z} 3=\mathrm{Z} 9$

$7910 \quad \mathrm{Z} 4=\mathrm{Z} 0$

$7920 \mathrm{Zl}=\mathrm{Mr}$

$7930 \mathrm{Z2}=\mathrm{Mi}$

7940 CALL Cdia(z1,Z2,Z3,Z4,Z9,Z0)

$7950 \mathrm{~S} 22 \mathrm{r}=\mathrm{Z} 9$

7960 S22 $i=Z 0$

$7970 \mathrm{X}=\mathrm{Z} 9$

$7980 \mathrm{Y}=\mathrm{ZO}$

7990 CALL Crpa $(X, Y, R, T)$

$8000 \mathrm{~S} 22 \mathrm{M}=\mathrm{R}$

8010 S22a $=T$

8020 !

$8030 ! S 12^{\wedge} 2=\left(a^{\prime}-b c^{\prime}\right) /\left(a-b^{\prime} c\right)+S 11 * S 22$

8040 !

8050 !

$8060 \mathrm{Z3}=\mathrm{C} 11(2)$

$8070 \mathrm{Z} 4=\mathrm{C} 22(2)$

$8080 \mathrm{Z} 1=\mathrm{B} 11(1)$ 


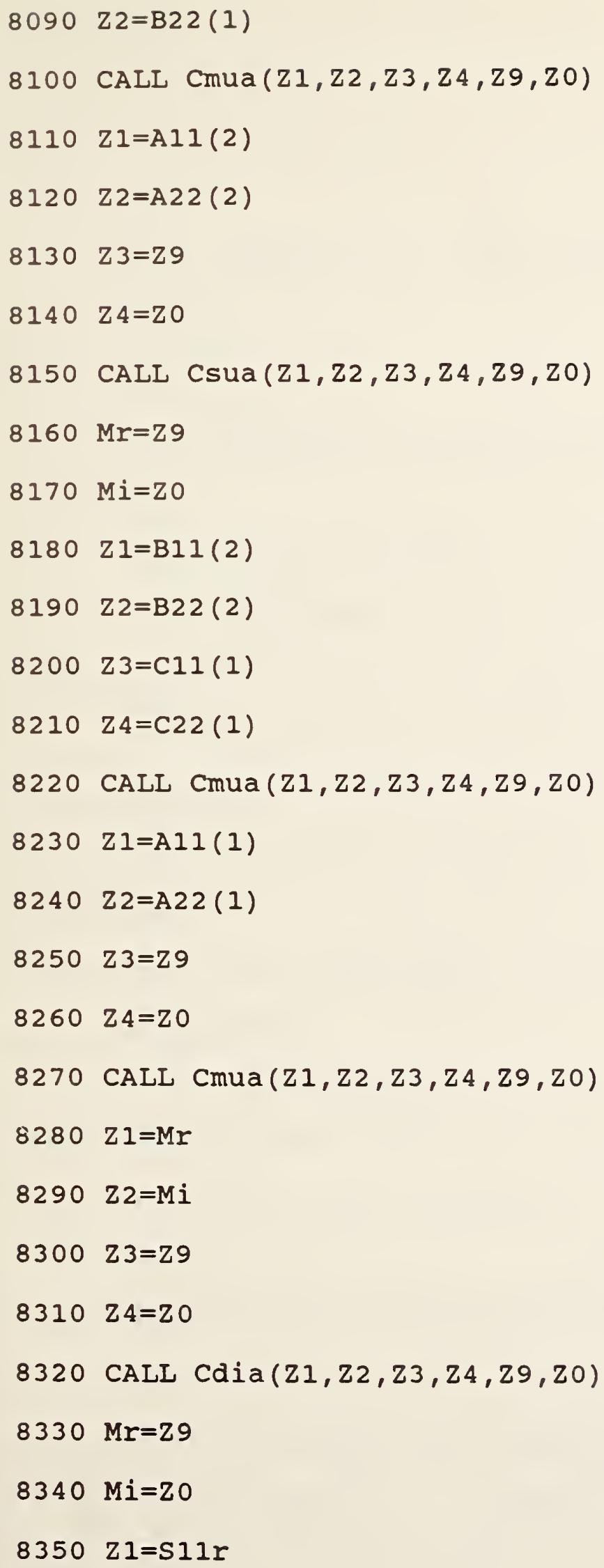


$8360 \mathrm{Z2}=\mathrm{S} 11 \mathrm{i}$

$8370 \mathrm{Z3}=\mathrm{S} 22 \mathrm{r}$

$8380 \quad Z 4=S 22 i$

8390 CALL Cmua $(\mathrm{Z} 1, \mathrm{Z2}, \mathrm{Z3}, \mathrm{Z4}, \mathrm{Z9}, \mathrm{Z0})$

$8400 \quad \mathrm{Z} 1=\mathrm{Z} 9$

$8410 \quad \mathrm{Z} 2=\mathrm{Z} 0$

$8420 \quad \mathrm{Z} 3=\mathrm{Mr}$

$8430 \quad Z 4=M i$

8440 CALL Cada $(Z 1, Z 2, Z 3, Z 4, Z 9, Z 0)$

$8450 \quad Z 1=Z 9$

$8460 \quad \mathrm{Z} 2=\mathrm{Z} 0$

8470 CALL Csqra (Z1,Z2,Z9,Z0)

8480 S $12 r=Z 9$

$8490 \mathrm{~S} 12 \mathrm{i}=\mathrm{ZO}$

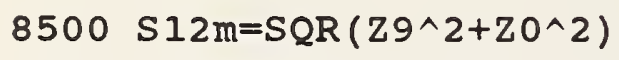

$8510 \mathrm{~S} 12 \mathrm{a}=\mathrm{ATN}(\mathrm{S} 12 \mathrm{i} / \mathrm{S} 12 \mathrm{r}) * 180 / \mathrm{PI}$

8520 PRINT "S12m,S12a,S12r,s12i"

8530 PRINT S12m,S12a,S12r,S12i

$8540 !$

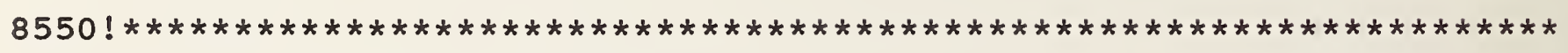

8560 ! Alpha $210=(S 21 \mathrm{~m}) \wedge 2 /\left(1-S 22 \mathrm{~m}^{\wedge} 2\right)$

8570 ! Alpha $120=(\mathrm{S} 21 \mathrm{~m}) \wedge 2 /\left(1-\mathrm{S} 11 \mathrm{~m}^{\wedge} 2\right)$

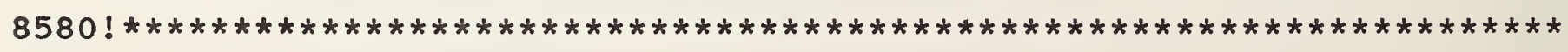

$8590 \mathrm{~A} 120=\mathrm{S} 12 \mathrm{~m}^{\wedge} 2 /\left(1-\operatorname{S} 11 \mathrm{~m}^{\wedge} 2\right)$

$8600 \mathrm{~A} 210=S 12 \mathrm{~m}^{\wedge} 2 /\left(1-S 22 \mathrm{~m}^{\wedge} 2\right)$

$8610 \mathrm{Db} 120=10 * \operatorname{LGT}(\mathrm{A} 120)$

$8620 \mathrm{Db} 210=10 * \operatorname{LGT}($ A2 10)

8630 PRINTER IS 16 
8640 PRINT

8650 FIXED 0

8660 PRINTER IS 0

8680 PRINT

8690 PRINT "REFLECTION COEFFICIENTS FOR ADAPTER ON SIX-PORT \#";SPOI

8691 FIXED 2

8700 PRINT

8701 PRINT"FREQUENCY" ;Freqs (P) ; "GHZ"

8702 PRINT

8710 PRINT "SII MAGNITUDE

S11 ANGLE

SII REAL

S11

IMAGINARY"

8720 PRINT

$-------n^{\prime \prime}$

8730 FIXED 4

8740 PRINT SIIm, SIIa, S11r,S11i

8750 PRINT

8760 PRINT "S22 MAGNITUDE

S22 ANGLE

S22 REAL

522

IMAGINARY"

8770 PRINT

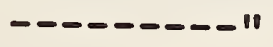

8780 PRINT S22m,s22a,s22r,s22i

8790 PRINT

8791 PRINT

8800 FIXED 0

8810 PRINT "LOSS DATA FOR ADAPTER ON SIX-PORT";SPORT

8820 PRINT "Alpha 12

LOSS DB

Alpha 21

LOSS DB"

8830 PRINT"-- 
8840 FIXED 4

8860 PRINT A120, Db120, A210, Db210

$8870 \mathrm{~K}=0$

8880 PRINT

8900 NORMAL

8920 IF sport $=2$ THEN $\mathrm{K}=17$

8921 IF sport=1 THEN $\mathrm{K}=0$

$8930 S(P, K+1)=S 11 m$

$8940 S(p, K+2)=S 11 a$

$8950 \mathrm{~S}(\mathrm{P}, \mathrm{K}+3)=\mathrm{S} 11 \mathrm{r}$

$8960 S(P, K+4)=S 11 i$

$8970 S(P, K+5)=A 120$

$8980 S(P, K+6)=S 22 m$

$8990 \mathrm{~S}(\mathrm{P}, \mathrm{K}+7)=\mathrm{S} 22 \mathrm{a}$

$9000 S(P, K+8)=S 22 r$

$9010 S(P, K+9)=S 22 i$

$9020 \mathrm{~S}(\mathrm{P}, \mathrm{K}+10)=\mathrm{A} 120$

$9030 S(P, K+11)=S 12 m$

$9040 S(P, K+12)=S 21 m$

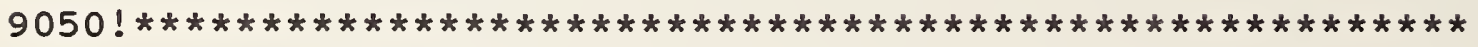

9060 PRINTER IS 16

9070! THE FOLLOWING CODE EXISTS TO CALCULATE EFFICIENCY OF ADAPTER AND D.U.T.

$9080 !$

$9090 !$

$9100 ! \mathrm{G} 1=\mathrm{S} 11+(\mathrm{S} 12 * \mathrm{~S} 21 * \mathrm{GX}) /(1-\mathrm{S} 22 * \mathrm{GX})$

$9110 !$

9111 ON Sport GOTO 91209132 
$9120 \mathrm{R}=\mathrm{Gxm}($ Sport $)=\mathrm{M} 1$

$9130 \mathrm{~T}=\mathrm{Gxa}($ sport $)=\mathrm{P} 1$

9140 CALL Cpra $(R, T, X, Y)$

$9150 \operatorname{Gxr}($ Sport $)=\mathrm{X}$

$9160 \mathrm{Gxi}($ Sport $)=Y$

$9210 \mathrm{ZI}=\mathrm{Z} 3=\mathrm{S} 12 \mathrm{r}$

$9220 \mathrm{Z2}=\mathrm{Z} 4=\mathrm{S} 12 \mathrm{i}$

9230 CALL Cmua $(Z 1, Z 2, Z 3, Z 4, Z 9, Z 0)$

$9240 \quad Z 1=Z .9$

$9250 \quad \mathrm{Z} 2=\mathrm{ZO}$

$9260 \mathrm{Z3}=\mathrm{Gxr}$

$9270 \quad Z 4=G \times i$

9280 CALL Cmua $(Z 1, Z 2, Z 3, Z 4, Z 9, Z 0)$

9290 Nur $=Z 9$

$9300 \mathrm{Nui}=\mathrm{ZO}$

9310 PRINT Nur, Nui, Z9, Zo

$9320 \mathrm{Z} 1=\mathrm{S} 22 \mathrm{r}$

$9330 \mathrm{Z2}=\mathrm{S} 22 \mathrm{i}$

9340 Z3=GXm (Sport)

$9350 \quad Z 4=0$

9360 CALL Cmua $(Z 1, Z 2, Z 3, Z 4, Z 9, Z 0)$

9370 PRINT "S22GX"

9380 PRINT Z9,ZO

$9390 \quad Z 1=1$

$9400 \quad \mathrm{Z} 2=0$

$9410 \quad Z 3=Z 9$

$9420 \quad Z 4=Z 0$ 
9430 CALL Csua (Z1, Z2, Z3, Z4, Z9, Z0)

9440 PRINT "1-S22GX",Z9,Z0

$9450 \quad \mathrm{Zl}=$ Nur

$9460 \mathrm{Z2}=\mathrm{NuI}$

$9470 Z 3=Z 9$

$9480 \quad \mathrm{Z} 4=\mathrm{Z} 0$

$9490 \mathrm{Mag} 1=\mathrm{SQR}\left(\mathrm{Z9}{ }^{\wedge} 2+\mathrm{Z} 0 \wedge 2\right) \wedge 2$

9500 CALL Cdia $(\mathrm{Z1}, \mathrm{Z2}, \mathrm{Z3}, \mathrm{Z4}, \mathrm{Z9}, \mathrm{Z0})$

$9510 \mathrm{Z1}=\mathrm{S} 21 \mathrm{r}$

$9520 \quad \mathrm{Z} 2=521 i$

9530 PRINT "SIIR, S11I"; S11r,S11i

$9550 \quad Z 3=Z 9$

$9560 \quad Z 4=Z 0$

9570 CALL Cada $(\mathrm{Z} 1, \mathrm{Z2}, \mathrm{Z3}, \mathrm{Z4}, \mathrm{Z9}, \mathrm{Z0})$

9580 GIr=Z9

$9590 \mathrm{G} 1 \mathrm{i}=\mathrm{ZO}$

$9600 !$

$96 \$ 0 ! !$

$9620 !$ Alpha12 $=\left[\left(1-G \times m^{\wedge} 2\right) * S 12 m^{\wedge} 2\right] /\left[1-G 1 m^{\wedge} 2 *(1-S 22 G x) m^{\wedge} 2\right]$

9630 !

$\left.9660 \mathrm{Magn}=\left(1-\mathrm{GXm}^{\wedge} 2\right) * \mathrm{~S} 12 \mathrm{~m}^{\wedge} 2\right)$

$9670 \mathrm{GIm}=\operatorname{SQR}\left(\mathrm{GIr}^{\wedge} 2+\mathrm{GIi \wedge} 2\right)^{\wedge} 2$

$9680 \mathrm{X}=\mathrm{G} 1 \mathrm{r}$

$9690 \quad Y=G 1 i$

9700 CALI Crpa $(X, Y, R, T)$

9710 Gla=T

9720 Magd $=(1-G 1 \mathrm{~m}) *$ Magl

9730 Alpha12=Magn/Magd 
9740 A12=Alpha12

9750 PRINTER IS 16

9760 PRINT "GIm, GIa, GIr, GIi"

9770 Print SQR(GIm), GIa,GIr,GIi

9780 PRINT "Gxm, Gxa, Gxr, Gxi"

9790 PRINT Gxm(Sport), Gxa(Sport), Gxr(Sport), Gxi (sport)

9801 PRINTER IS 0

9805 PRINT "EFFICIENCY OF ADAPTER AND DEVICE COMBINATION";A12

9806 PRINT

9807 PRINT

9808 PRINTER IS 16

9810 IF sport=1 then GOTO 9830

9920 GO TO 9900

$9830 \mathrm{~S}(\mathrm{P}, \mathrm{K}+13)=\mathrm{GxM}(\mathrm{Sport})$

$9840 \mathrm{~S}(\mathrm{P}, \mathrm{K}+14)=\mathrm{G} \times \mathrm{a}($ Sport $)$

$9850 S(P, K+15)=G \times r($ Sport $)$

$9860 \mathrm{~S}(\mathrm{P}, \mathrm{K}+16)=\mathrm{Gxi}(\mathrm{Sport})$

$9861 \mathrm{~S}(\mathrm{P}, \mathrm{K}+17)=\mathrm{A} 12$

9890 GOTO 9940

$9900 \mathrm{~S}(\mathrm{P}, \mathrm{K}+13)=\mathrm{Gxm}$ (Sport)

$9910 \mathrm{~S}(\mathrm{P}, \mathrm{K}+14)=\mathrm{G} \times \mathrm{a}$ (Sport)

$9920 \mathrm{~S}(\mathrm{P}, \mathrm{K}+15)=\mathrm{Gxi}$ (Sport)

$9930 \mathrm{~S}(\mathrm{P}, \mathrm{K}+16)=\mathrm{G} \times \mathrm{r}(\mathrm{Sport})$

9940 sport=sport +1

9950 if sport=2 THEN GOTO 6790

9951 PRINT PAGE

9952 sport $=1$ 


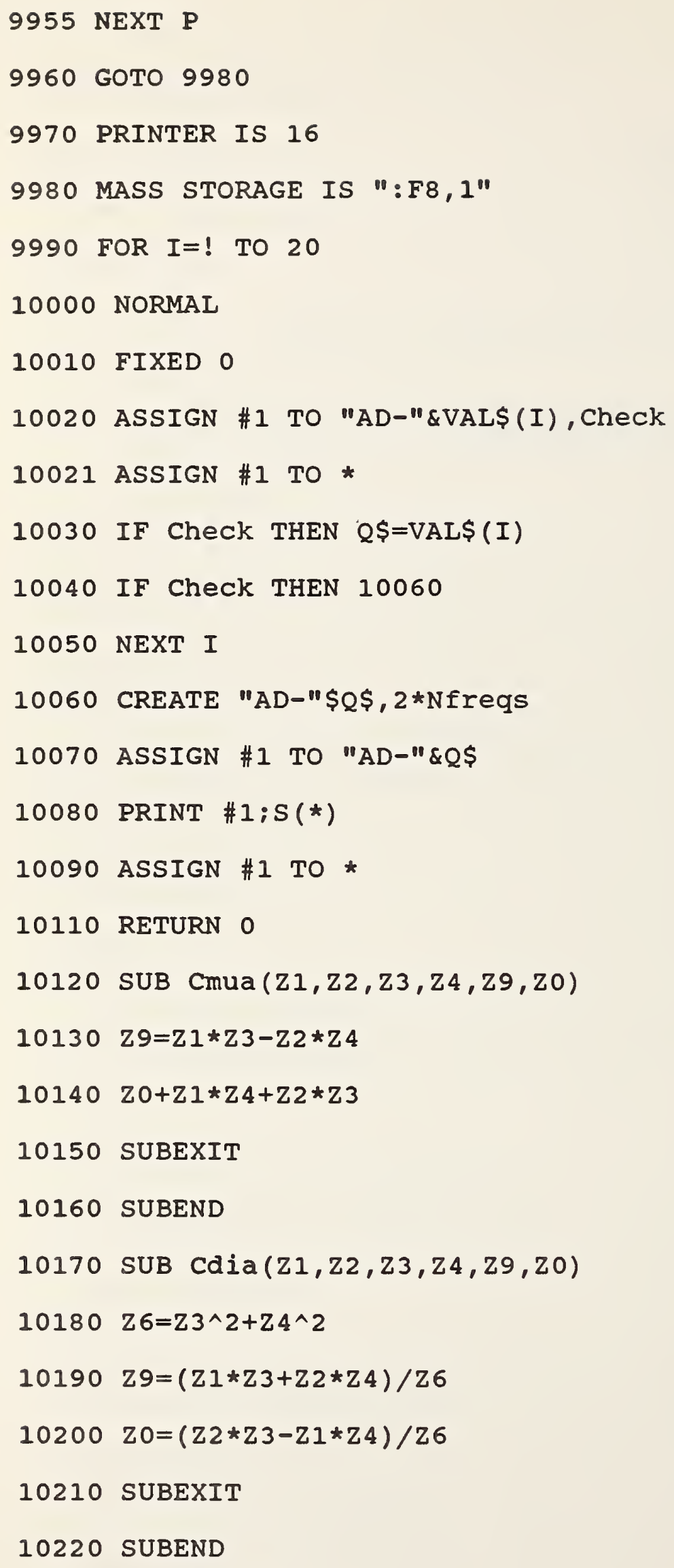




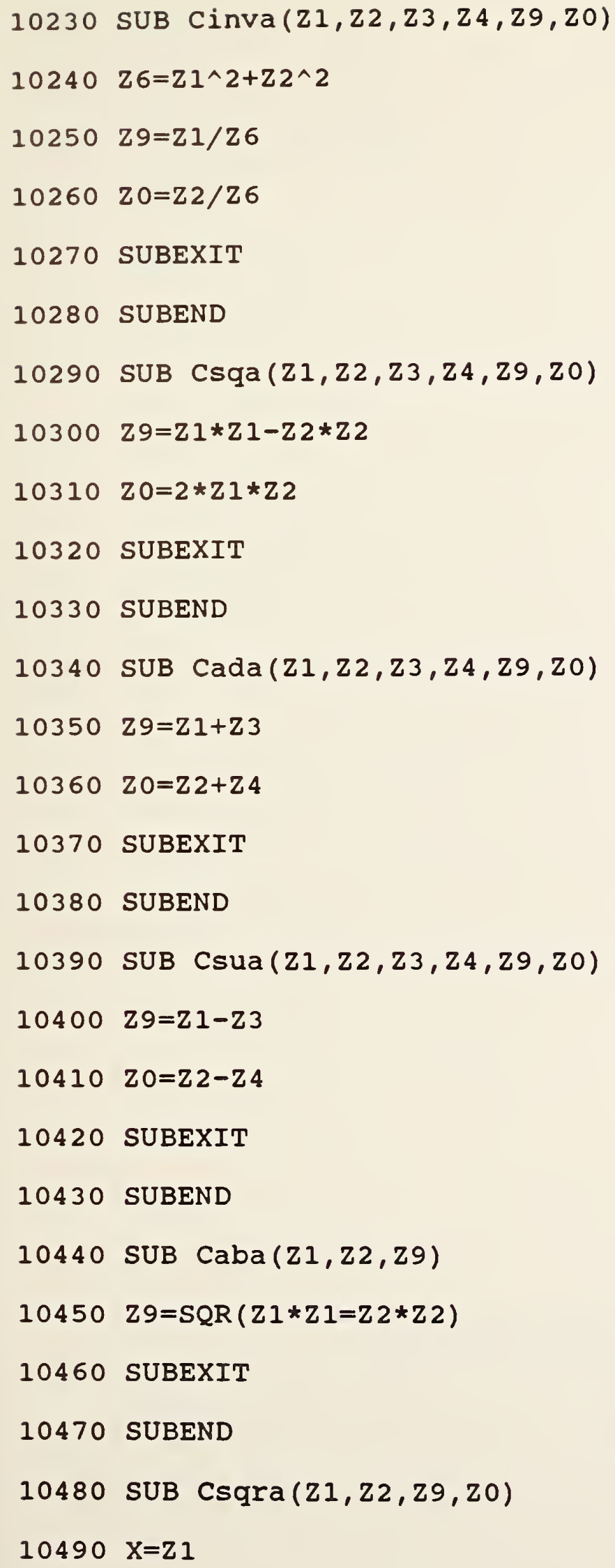


$10500 \quad \mathrm{Y}=\mathrm{Z} 2$

$10510 \operatorname{CALL} \operatorname{Crpa}(\mathrm{X}, \mathrm{Y}, \mathrm{R}, \mathrm{T})$

$10520 R=S Q R(R)$

$10530 \mathrm{~T}=\mathrm{T} / 2$

$10540 \mathrm{Z} 9=\mathrm{R} * \operatorname{COS}(\mathrm{PI} * \mathrm{~T} / 180)$

$10550 \mathrm{ZO}=\mathrm{R} * \mathrm{SIN}(\mathrm{PI} * \mathrm{~T} / 180)$

10560 GOTO 12570

10570 SUBEXIT

10580 SUBEND

10590 SUB Crpa $(X, Y, R, T)$

10600 $R=S Q R\left(X^{\wedge} 2+Y^{\wedge}\right)$

10610 IF $X=0$ THEN GO TO 10640

$10620 \mathrm{~T}=\mathrm{ATN}(\mathrm{Y} / \mathrm{X})$

10630 GO TO 10650

$10640 \mathrm{~T}=\mathrm{PI}$ *SGN $(\mathrm{Y}) / 2$

$10650 \mathrm{~T}=\mathrm{T} * 180 / \mathrm{PI}$

10660 IF $X>=0$ THEN 10710

10670 IF $Y>=0$ THEN 10700

$10680 \mathrm{~T}=\mathrm{T}-180$

10690 GO TO 10710

$10700 \mathrm{~T}=\mathrm{T}+180$

10710 SUBEXIT

10720 SUBEND

10730 SUB cmuada $(\mathrm{z} 1, \mathrm{z} 2, \mathrm{z} 3, \mathrm{z} 4, \mathrm{z} 5, \mathrm{z} 6, \mathrm{z} 7, \mathrm{z} 8, \mathrm{z} 9, \mathrm{z} 0)$

$10740 \mathrm{Z9}=\mathrm{Z} 1 * \mathrm{Z} 3-\mathrm{Z} 2 * \mathrm{Z} 4+\mathrm{Z} 5 * \mathrm{Z} 7-\mathrm{Z} 6 * \mathrm{Z} 8$ )

$10750 \mathrm{z} 0=\mathrm{Z} 1 * \mathrm{Z} 4-\mathrm{z} 2 * \mathrm{Z} 3+\mathrm{Z} 5 * \mathrm{Z} 8+\mathrm{Z} 6 * \mathrm{Z} 7)$

10760 SUBEXIT

10770 SUBEND 
10780 SUB Cpra $(R, T, X, Y)$

$10790 \mathrm{X}=\mathrm{R} 8 \cos (\mathrm{PI} \star \mathrm{T} / 180)$

$10800 \mathrm{Y}=\mathrm{R} \star \mathrm{SIN}(\mathrm{PI} * \mathrm{~T} / 180)$

10810 SUBEXIT

10820 SUBEND 


\section{APPENDIX II}

LISTING OF ADAPTER PARAMETER CALCULATING SUBPROGRAM "Gamma"

11300 SUB Gamma (Nfreqs, Repeats,Ca\$)

11310 OPTION BASE 1

11320 DIM Power(Nfreqs, Repeats, 16,10), Po (1,8), Result (Nfreqs, 60) Wrspt $(2,12)$

11330 FREAD "1PRD\&"3"\&":F8, 1",Power(*)

$11340 \mathrm{G}=1$

11350 PRINTER IS 16

11360 File\$="CRST" \&"2"

11370 FOR F=1 TO Nfreqs

11380 CALL Redwrt(File\$,"F8, 1"," ",Result(*), Nfreqs)

11390 FOR $J=1$ TO 2

11400 FOR N=1 TO 12

11410 PRINTER IS 0

$11420 \operatorname{Wrspt}(J, N)=\operatorname{Result}(F, N+(J-1) * 12)$

11430 NEXT N

$11440 \mathrm{NEXT} J$

11480 FOR Mnt $=1$ to 8

11490 Po $(1$, Mnt+Inc $)=\operatorname{Power}(F, 1,1$, Mnt+Inc $)$

11500 NEXT Mnt

$11520 \operatorname{CALL} \operatorname{Mg}(\mathrm{Po}(*), 1,1, \operatorname{Wrspt}(*), " \mathrm{P} ", \mathrm{M1}, \mathrm{P1}, \mathrm{R} 1)$

11530 CALL Mg(Po(*), 1, 1, Wrspt (*), "P" , M1, P1, R2)

11540 PRINTER IS 0

11550 PRINT "FREQ\#", F

11552 PRINT 
11554 PRINT "DEVICE PLUS ADAPTER ON 6-PORT 1"

\section{PRINT}

11560 PRINT "GAMMA MAGNITUDE", "GAMMA PHASE"

11561 PRINT "----

11562 PRINT USING $11585 ; \mathrm{MI}, \mathrm{PI}$

\section{PRINT}

11564 PRINT "DEVICE PLUS ADAPTER ON 6-PORT 2"

11565 PRINT

11567 PRINT "GAMMA MAGNITUDE","GAMMA PHASE"

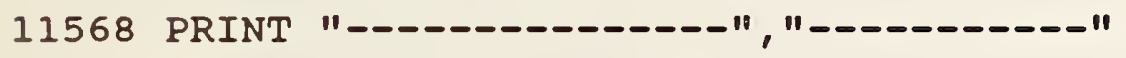

11570 PRINT USING $11585 ; \mathrm{M} 2, \mathrm{P} 2$

11585 IMAGE MD. DDDDD, 12X, MDDD. DDDD

11590 NEXT F

11591 NORMAL

11592 PRINT

11593 PRINT

11594 PRINT

11595 PRINTER IS 16

11600 Q=FNAdp (Nfreqs, Repeats, M1, P1 , M2 , P2 , Ca\$)

11610 SUBEXIT

11620 SUBEND 


\section{APPENDIX III}

\section{COMPLETE LISTING OF MEASUREMENT PROGRAM "ADP5M"}

\section{9}

\section{ADP5M}

$3 ! * * * * * *$ ADP5M $* * * * * *$

4 ! RE-STORE "ADP5M:F8" !AUGUST 28, 19851020

6 ! FILE NAME: ADPTR5 !850828 0804 BCY-GJC ORIG VS IS CALBR40205-1000---BCY

7 ! ADPII INSERTED AT LINE 9000 THIS VERSION

9 OPTION BASE 1

12 DIM F list(11),Path(11),Amps (5), Line(11),Fstop a (11),Famps(6)

13 DIM Tȳp\$[6], Fil nam\$[6], Linput\$[160], Mntno(10)

14 ! CALL DOC

22 PRINTER IS 16

23 DISP " "

24 Max pts $=11$

27 Nireqs $=0$

$28 \mathrm{Gl}=0$

30 Typ=1

$31 \mathrm{Cal}=1$

33 ON ERROR GOTO Off

36 Tape $\$=" 14$ "

39 MASS STORAGE IS ":T"\&Tape\$

40 MASS STORAGE IS ":F8, 1 "

42 Print $\$=" 0 "$

45 OFF ERROR

48 GOTO Freq

51 Off: OFF ERROR

54 Print $\$=17,1 "$

57 GOTO Freq

60 Task: PRINT CHR\$(12); LIN(5);TAB(10);CHR\$(7);"ENTER OPTION NUMBER FOR NEXT OPERATION:"

61 IF PIOC<O THEN INPUT "NUMBER OF FREQUENCIES TO PROCESS AND NUMBER OF Repeats", Nfreqs, Repeats

63 GOSUB Menu

66 Opt: LINPUT "OPTION NUMBER ? ", Linput\$

69 IF NOT FNNumi(Linput\$) THEN Opt

72 Typ=VAL (Linput\$)

IF $($ TYP $<0)$ OR $($ TYP>5) THEN Opt

IF Typ $>2$ THEN Typ=Typ+1

IF TYP $>5$ THEN End

IF PrOC $=-1$ THEN PrOC $=0$

IF TYp=5 THEN CALL Gamma(Nfreqs, Repeats, Ca\$)

IF TYP=5 THEN GOTO 22

81

83 Freq:IF Typ=1 THEN CALL Freqin(Max pts, Nfreqs, Hi_lo_port, F_list (*), Path (*), Amps (*), Liñe(*), Lines, Fstop_a (*), Famps (*), Proc) 
GOTO Task

IF TYP>1 THEN TYP

90

93

TYP: PRINT CHR\$(7); IIN(5) ;TAB(10);"TURN ON AMPLIFIER(S):" FOR $I=1$ TO 5

96 IF Amps(I) THEN PRINT TAB(15) ;Famps(I);"-";Famps (I+1) NEXT I

PRINT LIN (2) :TAB (10);"PRESS 'CONT' WHEN READY"

PAUSE

108

Dim=16* $($ Typ $<3)+4 *($ TYp $=3)+($ Typ $>3)+($ TYp $=5)$

IF TYP=1 THEN TYP\$="FREQ"

IF TYP=2 THEN TYP\$="PRTCAL"

114

115

IF TYP=4 THEN TYP\$="1_PORT"

117

cnnct: PRINT CHR\$(7); $\operatorname{CHR} \$(12) ; \operatorname{LIN}(5) ; T A B(10) ; " E N T E R$ CONNECTOR TYPE OPTION:"

118

119

120

121

122

123

124

126

129

132

135

138

141

144

147

150

151

153

156

159

162

163

164

165

168

169

IF $\mathrm{Cal}=4$ THEN $\mathrm{Cal}=\mathrm{VAL}$ ( $\mathrm{Ca} \$$ )

\section{PRINT}

IF ( $\mathrm{Cal}=2$ ) AND (TYP=4) THEN PRINT "

ADAPTER AND 1 PORT DEVICE"; CHR\$ (7)

IF $(\mathrm{Cal}=2)$ AND $($ TYp $<4)$ THEN PRINT " iCHR\$ (7)

\section{PRINT}

GOSUB MenuI

LINPUT "ENTER OPTION NUMBER ", Linput\$

IF NOT FNNumi(Linput\$) THEN cnnct

Cnnct $=$ VAL ( Linput $\$$ )

IF (Cnnct<1) OR (Cnnct>3) THEN Cnnct

IF Cnnct=1 THEN Cnnct $\$=" N O T$ N"

IF Cnnct $=2$ THEN Cnnct $\$=" T N$ 2L"

IF cnnct=3 THEN Cnnct $\$=" T N-2 R "$

IF Cnnct $=4$ THEN cnnct $\$=" W G \bar{N} "$

Rep2: PRINT CHR\$(12);CHR\$ (7)

LINPUT "ENTER \# OF REPEAT MEASUREMENTS (SIDE-ARM SCANS) -- $(1-10)$ ", Linput\$

\section{Linput $\$=" 1 "$}

Tst: IF NOT FNNumi(Linput\$) THEN Rep2

Repeats $=$ VAL (Linput $\$$ )

IF (Repeats $<1$ ) OR (Repeats $>10$ ) THEN Rep2

IF (Repeats $>3$ ) AND (TYP<3) THEN Rep2

!IF (PrOC=1) AND (TYP $=4$ ) THEN GOTO Gammal

IF PrOC>0 THEN GOTO 171

Cal: DISP

CALL Caldat (Max pts, Nfregs, Repeats, Dim,Hi_lo_port, F_list (*), Typ\$,S_port,std_mnt,Print\$,PaEh (*), Line(*), Lines, Fstop a (*), Cnncts, Mounts, Mntno (*), Cal)

171 CALL Calres (Max_pts, Nfreqs, Repeats, Dim, Typ\$, S_port, Std_mnt,Hi_lo_port, Print\$, Cal,Gl, Ca\$)

174 DISP " "

177 DISP "DO YOU WANT TO SAVE SYSTEM PARAMETER DATA ON

178 Flag=FNYOrn MASS STORAGE ?"; CH\$(7)

180 IF NOT Flag THEN GOTO Jump225

183 DISP " "

186 DISP "INSERT USER DISC INTO MASS STORAGE F8, 0 --- PRESS 'CONT' WHEN READY";CHR\$(7) 
File:

DISP " ";CHR\$ (7)

195

LINPUT

"ENTER USER FILE CHARACTERS", Fil_nam\$

198 IF LEN(Fil_nam\$)>6 THEN DISP "FILE NAME IS TOO LONG" ; CH R $\$(7)$;

201 IF LEN(Fil_nam\$) $>6$ THEN File

204 ASSIGN \# I TO Fil_nam\$\&":F8,0", Check

207 ASSIGN * TO \#1

210 IF Check THEN Disc

213 DISP " "

216

219

DISP "FILE NAME ";Fil nam\$;" IS IN USE. CONTINUE -- $(\mathrm{Y} / \overline{\mathrm{N}})$ ?"; $\operatorname{CHR} \$(7)$;

219 FNYOI THEN Flag=1

220 IF Flag THEN PURGE Fil_nam $\$$ \&":F8, 0"

221

222

223

224

225

226

228

230

231

234

235

236

237

238

239

240

243

244

245

246

249

252

255

IF Flag THEN Disc

GOTO File

IF $G 1=1$ THEN $C a \$=" 3 "$

Disc: CALL Disc_copy(Fil_nam\$,Nfreqs, Typ\$,Ca\$)

Ca\$=VAL $\$(\mathrm{Cal})$

Jump2 25: IF Cal=4 THEN GOTO cnnct

IF Cal=1 THEN GOTO 237 ! ! ! ! ! ! ! ! ! ! ! ! ! ! ! ! ! ! ! ! ! ! ! ! ! ! ! !

IF $\mathrm{Cal}=3$ THEN Task

! Gamma (Nfreqs, Repeats, Ca\$)

GOTO 22

GOTO Task

$\mathrm{Cal}=1$

GOTO Task

$\mathrm{Cal}=2$

GOTO Cnnct

Menu: IF Proc $<0$ THEN CALL Gamma (Nfreqs, Repeats, Ca\$)

IF PrOC $<0$ THEN GOTO 48

PRINT USING Form 1

PRINT USING FOrm2

RETURN

Menul: PRINT USING Form3

PRINT USING FOIm 4

RETURN

FOIM1: IMAGE /10X"1) INPUT FREQUENCIES"/10X"2)

DETERMINE SYSTEM PARAMETERS WITHOUT POWER STANDARD"

258 FOrm2: IMAGE 10X"3) MEASURE 1-PORT ON ADAPTER"/10X"4) EVALUATE ADAPTERS"/10X"5) EXIT"

261 Form3: IMAGE / 10X"1) $14 \mathrm{~mm}, 7 \mathrm{~mm}$, OR WAVEGUIDE"/10X"2)

TYPE N, MALE ADAPTER ON 6-PORT \#1"/10X"3) TYPE N, MALE ADAPTER ON 6-PORT \#2"

264 Form4: IMAGE 1OX"4) OTHER, NOT AVAILABLE AT PRESENT"

267 End: DISP "MEASUREMENT SESSION DONE"

268 PRINT CHR\$(12)

270 Gammal:PRINT "THIS IS Gamma"! THIS SEGMENT MUST CALL DATA FILES NEEDED

271 PAUSE

272 CALL Caldat (Max_pts, Nfreqs, Repeats, Dim, Hi lo_port, F_list (*), Typ\$,s_port, std_mnt, Print\$,Path (*), Line (*) , Lines, Fstop_a $(*)$, CnncEs, Mounts, Mntno $(*), \mathrm{Cal})$

273 CALL Gamma(Nfregs) 
274 Q=FNAdp (Nfreqs)

275 GOTO 235

276 END

279 ! FILE NAME: FREQIN

! 800610-1615---BCY

MAIN

Amps (

12

83

96

$\mathrm{Ca} \$$

80

118

171

223

224

225

239

Ca\$ :

$0 \quad 223$

Cal

$31 \quad 118$

$225 \quad 226$

118

120

121

168

237

171

$230 \quad 235$

272

Cal:

165

Check

$204 \quad 210$

Cnnct

$129 \quad 132$

132

135

138

141

144

cnnct\$

$0 \quad 135$

138

141

144

168

272

Cnnct\$:

$0 \quad 135$

138

141

144

Cnnct:

$117 \quad 126$

132

226

238

Dim

108

168

171

272

Disc:

$224 \quad 210 \quad 221$

End:

$267 \quad 78$

F_list(

12

83

168

272

Famps (

12

83

96

96

Fil_nam\$

$\begin{array}{llll}13 & 195 & 198 & 201\end{array}$

224

$192 \quad 201 \quad 222$

178

180

219

220

221

Form1:

255

Form2:

258

Form3:

261

Form4:

264

Freq:

83

48

57 


\begin{tabular}{|c|c|c|c|c|c|c|c|}
\hline Fstop_al & 12 & 83 & 168 & 272 & & & \\
\hline G1 & 28 & 171 & 223 & & & & \\
\hline Gamma 1: & 270 & & & & & & \\
\hline Hi_10_port & 83 & 168 & 171 & 272 & & & \\
\hline I & 93 & 96 & 96 & 96 & 99 & & \\
\hline Jump225: & 226 & 180 & & & & & \\
\hline Linel & 12 & 83 & 168 & 272 & & & \\
\hline Lines & 83 & 168 & 272 & & & & \\
\hline Linput\$ & $\begin{array}{l}13 \\
151\end{array}$ & $\begin{array}{l}66 \\
153\end{array}$ & $\begin{array}{l}69 \\
156\end{array}$ & 72 & 124 & 126 & 129 \\
\hline Max_pts & 24 & 83 & 168 & 171 & 272 & & \\
\hline Menul: & 246 & 123 & & & & & \\
\hline Menu: & 239 & 63 & & & & & \\
\hline Mntnol & 13 & 168 & 272 & & & & \\
\hline Mounts & 168 & 272 & & & & & \\
\hline Nfreqs & $\begin{array}{r}27 \\
239\end{array}$ & $\begin{array}{r}61 \\
272\end{array}$ & $\begin{array}{r}80 \\
273\end{array}$ & $\begin{array}{r}83 \\
274\end{array}$ & 168 & 171 & 224 \\
\hline Off: & 51 & 33 & & & & & \\
\hline Opt: & 66 & 69 & 75 & & & & \\
\hline Path ( & 12 & 83 & 168 & 272 & & & \\
\hline Print\$ & 42 & 54 & 168 & 171 & 272 & & \\
\hline Proc & 61 & 79 & 79 & 83 & 164 & 239 & 240 \\
\hline $\mathbf{Q}$ & 274 & & & & & & \\
\hline Rep2: & 147 & 153 & 159 & 162 & & & \\
\hline Repeats & $\begin{array}{r}61 \\
171\end{array}$ & $\begin{array}{r}80 \\
239\end{array}$ & $\begin{array}{l}156 \\
272\end{array}$ & 159 & 159 & 162 & 168 \\
\hline s_port & 168 & 171 & 272 & & & & \\
\hline std_mnt & 168 & 171 & 272 & & & & \\
\hline Tape\$ & 36 & 39 & & & & & \\
\hline
\end{tabular}


Task:

Tst:

Typ

Typ\$

Typ\$ :

Typ:
60

$87 \quad 230$

234

236

153

$\begin{array}{rr}30 & 72 \\ 78 & 80 \\ 108 & 108 \\ 162 & 169\end{array}$

13
272

0

111

114

115

90

84 
282 SuB Freqin(Max_pts, Nfreqs,Hi_lo_port, F_list(*), Path(*),

285 OPTION BASE 1

288 PRINTER IS 16

291 PRINT CHR\$(12)

294 DISP " "

297 Famps $(1)=.25$

300 Famps $(2)=1$

303 Famps $(3)=2$

306 Famps $(4)=4$

309 Famps $(5)=8$

312 Famps $(6)=18$

315 CALL Freq(Max_pts,Nfreqs, Hi_lo_port,F_list(*),Path(*),Amps(*), Line (*), Iines, Fstop_a( $\left.{ }^{(}\right), \bar{B}$ ails, Proc)

318 PRINT CHR\$(12)

321 DISP " "

324 SUBEND

327 ! $* * * \star * \star * * * * * * * * *$ SUBPROGRAM FREQ

330 ! FILE NAME: FREQ

! 811216-1715---BCY

s Freqin(

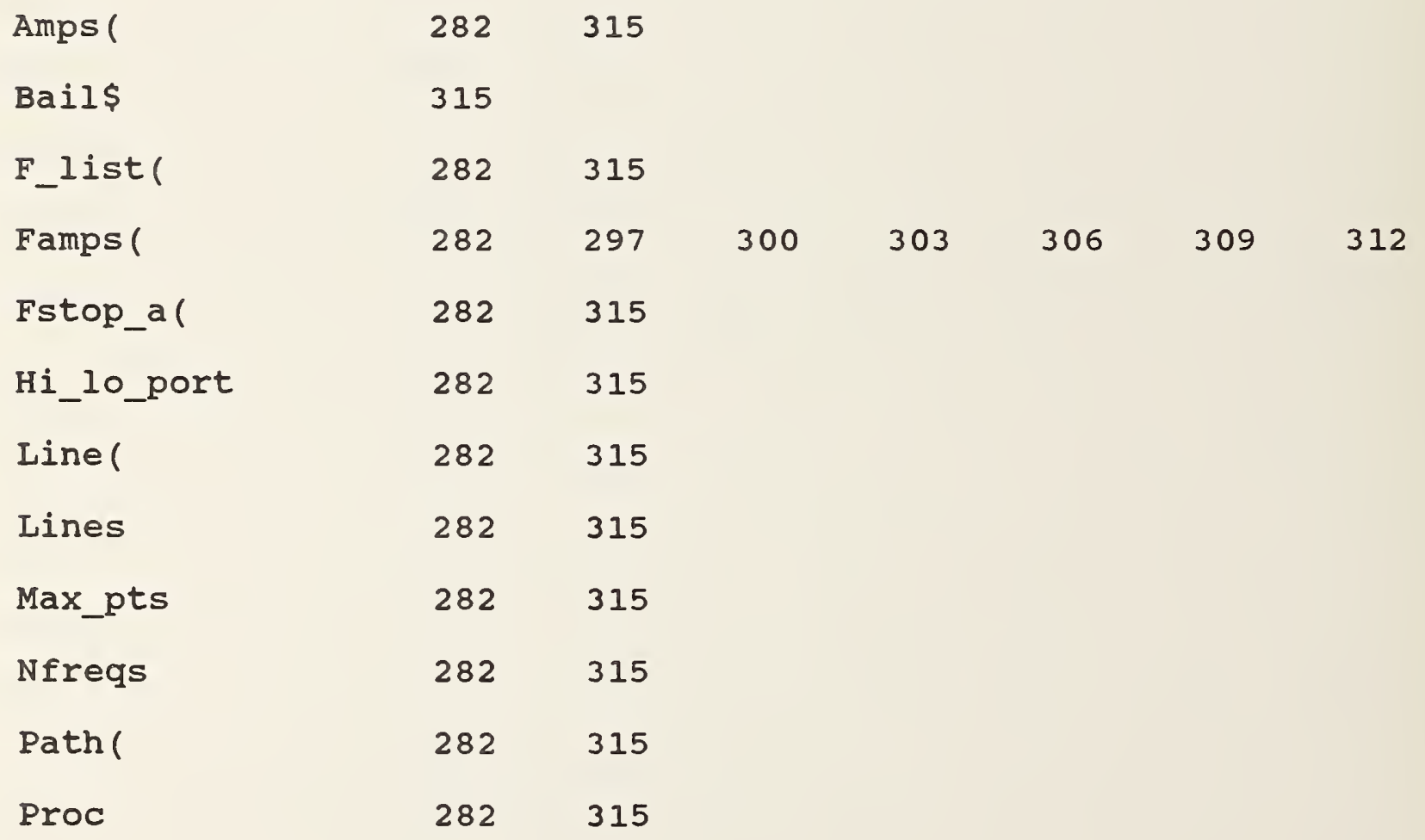


SUB Freq(Max pts, Nfreqs, Hi_10_port, F_list(*),Path(*),Amps(*), Line (*), Lines, Fstop_a( $\left.{ }^{(}\right), \bar{B}$ ail\$, Proc)

336

339

342

345

348

351

354

357

360

361

363

366

367

368

369

370

371

373

375

378

381

384

387

390

393

396

399

402

405

408

411

414

417

420

423

426

427

428

429

431

432

435

438

441

444

447

450

453

456

OPTION BASE 1

DIM A $[32]$, Linput $\$ 160]$

A $\$="$ FREQUENCY INPUT/OUTPUT SECTION"

Center $=(80-\operatorname{LEN}(\operatorname{TRIM} \$(A))) / 2$

PRINT TAB (Center), A\$, IIN (2);CHR\$ (7)

PRINT "MAXIMUM \# OF FREQUENCIES =" ; Max_pts

P_opt1: PRINT USING FOrm I;CHR\$ $(7)$

LINPUT "INPUT OPTION \# ", Linput\$

IF Linput $\$=" 5 "$ THEN GOTO 428

IF NOT FNNumi(Linput\$) THEN P_optI

Opt $=V A L$ ( Linput $\$$ )

IF VAL(Iinput $\$$ ) $=3$ THEN Proc $=1$

IF VAL (Linput $\$$ ) $=4$ THEN PrOC $=-1$

IF PrOC<O THEN GOTO 431

Opt $=1$

IF (Opt<=0) OR (Opt>2) THEN P_opt 1

P_opt2: PRINT USING FOrm2; CHR\$ (7)

LINPUT "INPUT 6-PORT OPTION \# ",Linput\$

IF NOT FNNumi(Linput\$) THEN P_opt2

S_opt $=$ VAL (Linput\$)

IF $\left(\bar{S}_{-}\right.$opt $\left.<=0\right)$ OR $\left(S_{-}\right.$opt $\left.>2\right)$ THEN P_opt2

Hillo_port=S_opt $=1$

Opt: $\quad$ Bail $\$=" N "$ PRINT CHR $(1 \overline{2})$

Flag=0

ON Opt GOSUB SSS, TYpe

IF Bail\$="Y" THEN GOSUB Restart

IF Flag THEN Opt

IF NOT Flag AND (Bail\$="Y") THEN Exit

! - - - - PROCESS DATA, WHICH AIR LINE, AMPLIFIER --.--

Round: $\quad I=I+1$

F_list $(I)=$ PROUND $\left(F \_\right.$ist $\left.(I),-6\right)$

$I \bar{F}$ I $<$ N freqs THEN Round

CALL Aline(Hi_lo_port, Nfregs, F_list(*), Line(*), Lines, Fstop_a( $\overline{(})$ )

GOTO 431

CALL Path $(\overline{\mathrm{H}}$ i_lo_port,Nfreqs, F_list $(*), \operatorname{Amps}(*), \operatorname{Path}(*))$

PRINT "END OF SESSION"

STOP

EXit: SUBEXIT

! -

Sss: CALL F_step(Opt,Hi_lo_port,Max_pts,Nfreqs, F_list(*), Báils, Proc)

RETURN

! -

Type: CALL F_type(opt,Hi_lo_port, Max_pts, Nfregs, F_list( $\left.{ }^{*}\right)$, Bāil\$, Proc)

RETURN

!

Restart: DISP "RESTART SAME OPTION ?-- $(\mathrm{Y} / \mathrm{N})$ "; CHR $(7) ;$ ! retry same option ?

IF FNYOrn THEN Flag=1 
459 RETURN

$462 !-\ldots-0 .-1 M A G E$ FORMATS -

465 FOIM1: IMAGE /"OPTIONS:"//"1. START,STOP,STEP LIST"/" 2. TYPE IN LIST", K!/"3. PROCESS DATA FOR ADAPT LOSS"/" 4. CALCULATE ADAPTER LOSS"/"5. EXIT" $/, \mathrm{K}$

468 FOrm2: IMAGE /"INPUT 6-PORT OPTION:"/"1) $<=2 \mathrm{GHz}$ 6-PORT"/"2) $>=2 \mathrm{GHz}$ 6-PORT"/, K

471 SUBEND

$474 ! * * * * * * * * * * * * * *$ SUBPROGRAM ALINE

477 ! FILE NAME: ALINE

! 811216-1600---BCY

S Freq(

\begin{tabular}{|c|c|c|c|c|c|c|c|}
\hline$A \$$ & 339 & 342 & 345 & 348 & & & \\
\hline Amps ( & 333 & 426 & & & & & \\
\hline Bails & 333 & 393 & 402 & 408 & 435 & 444 & \\
\hline Center & 345 & 348 & & & & & \\
\hline Exit: & 431 & 408 & & & & & \\
\hline F_list & 333 & 417 & 417 & 423 & 426 & 435 & 444 \\
\hline Flag & 396 & 405 & 408 & 456 & & & \\
\hline Forml: & 465 & & & & & & \\
\hline Form2: & 468 & & & & & & \\
\hline Fstop_al & 333 & 423 & & & & & \\
\hline Hi_10_port & 333 & 387 & 423 & 426 & 435 & 444 & \\
\hline$I$ & 414 & 414 & 417 & 417 & 420 & & \\
\hline Linel & 333 & 423 & & & & & \\
\hline Lines & 333 & 423 & & & & & \\
\hline Linput\$ & $\begin{array}{l}339 \\
375\end{array}$ & $\begin{array}{l}360 \\
378\end{array}$ & $\begin{array}{l}361 \\
381\end{array}$ & 363 & 366 & 367 & 368 \\
\hline Max_pts & 333 & 351 & 435 & 444 & & & \\
\hline Nfregs & 333 & 420 & 423 & 426 & 435 & 444 & \\
\hline Opt & 366 & 370 & 371 & 371 & 399 & 435 & 444 \\
\hline opt: & 393 & 405 & & & & & \\
\hline P_opt1: & 357 & 363 & 371 & & & & \\
\hline
\end{tabular}




$\begin{array}{lllllll}\text { P_opt2: } & 373 & 378 & 384 & & & \\ \text { Path( } & 333 & 426 & & & & \\ \text { Proc } & 333 & 367 & 368 & 369 & 45 & 44 \\ \text { Restart: } & 453 & 402 & & & & \\ \text { Round: } & 414 & 420 & & & & \\ \text { S_opt } & 381 & 384 & 384 & 387 \\ \text { Sss: } & 435 & 399 & & & \\ \text { Type: } & 444 & 399 & & \end{array}$


480 SUB Aline(Hi_lo_port,Nfreqs, F_list(*), Line(*), Lines, Fstop_a(*)) 483 OPTION BASE $\overline{1}$

486 DIM F ( 7 , Nfreqs)

489 Aline $(1)=15$

492 Aline (2) $=5$

495 Aline $(3)=3$

!. $25--<.75 \mathrm{GHz}$

! $.75--2 \mathrm{GHz}$

! $2--3.75,6.25--8.75,11.25--13.75$,

$16.25--18 \mathrm{GHz}$

Aline (4) $=5$

! >3.75--5.25,9.75--<13.75 GHz

Aline (5) $=6$

! $5.625--<6.25,>13.75--14.375$,

501

504

507

Aline $(6)=7.5 \quad !>8.75--9.375,14.5--15.5 \mathrm{GHz}$

$15.625--<16.25 \mathrm{GHz}$

Aline $(7)=10$

! >8.75--9.375, $14.5--15.5 \mathrm{GHz}$

$!>5.25--<5.625,>9.375--<9.75,>15.5$

$--<15.625 \mathrm{GHz}$

510

513

516

519

522

525

528

531

534

537

540

543

546

549

552

555

558

561

564

567

570

573

576

579

582

585

588

591

594

597

600

603

606

609

612

615

618

621

GOTO Inc 1

start $=\mathrm{J}=(\mathrm{Hi}$ 10_port $=1) * 2$

Stop $=2+($ Hi_IO_port $=1) * 5$

Hi_lo=Hi_lo_port+1

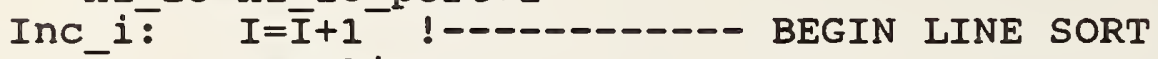

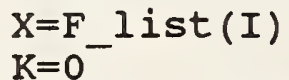

ON Hi 10 GOSUB LO, Hi

$\operatorname{Cntr}(\bar{K})=\operatorname{Cntr}(K)+1$

$\mathrm{F}(\mathrm{K}, \mathrm{I})=\mathrm{X}$

Inc_j: $\quad J=J+1$

IF J>Stop THEN SUBEXIT

IF $\operatorname{cntr}(J)=0$ THEN Inc_j

Inc_1: $\quad \begin{aligned} & \mathrm{L}=0 \\ & \mathrm{~L}=\mathrm{L}+1\end{aligned}$

IF L $>$ Nfregs THEN Inc_j

IF $F(J, L)=0$ THEN Inc_l

$\mathrm{N}=\mathrm{N}+1$

F_list $(\mathrm{N})=\mathrm{F}(\mathrm{J}, \mathrm{L})$

Fstop_a $(\mathrm{N})=\operatorname{Cntr}(\mathrm{J})$

Cnt=Aline $(\mathrm{J})$

Line $(\mathrm{N})=$ Cnt

IF cnt<>Last_cnt THEN Lines=Lines+1

Lo: $\quad \mathrm{K}=\overline{\mathrm{I}}$

RETURN

IF $\mathrm{X}>=.75$ THEN $\mathrm{K}=2$

$\mathrm{Hi}:$ IF $(\mathrm{X}>=2)$ AND $(\mathrm{X}<=3.75)$ OR $(\mathrm{X}>=6.25)$ AND $(\mathrm{X}<=8.75)$ OR $(X>=11.25)$ AND $(X<=13.75)$ OR $(X>=16.25)$ AND $(X<=18)$ THEN $\mathrm{K}=3$

IF K THEN RETURN

IF $(X>3.75)$ AND $(X<=5.25) \quad$ OR $(X>=9.75) \quad$ AND $(X<13.75)$ THEN $K=4$

IF $K$ THEN RETURN

IF $(X>=5.625)$ AND $(X<6.25)$ OR $(X>13.75)$ AND $(X<=14.375)$

OR $(X>=15.625)$ AND $(X<16.25)$ THEN $K=5$

IF $K$ THEN RETURN

IF $(X>8.75)$ AND $(X<=9.375) \quad$ OR $(X>=14.5) \quad$ AND $(X<=15.5)$

THEN $\mathrm{K}=6$

IF $K$ THEN RETURN

$\mathrm{K}=7$

RETURN 
624 SUBEND

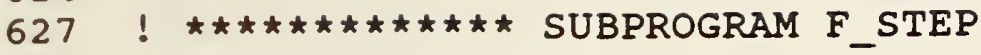

630 ! FILE NAME: F_STEP

! 811216-0830---BCY

S Aline(

\section{Alinel}

cnt

Cntr(

F (

F_list

Fstop_al

$\mathrm{Hi}$ :

Hi_lo

Hi_10_port

I

Inc_i:

Inc_j:

Inc_1:

$\mathrm{J}$

K

L

Last_cnt

Line(

Lines

Lo:

N

Nfreqs
489

570

570

531

486

480

480

$594 \quad 528$

$516 \quad 528$

$480 \quad 510$

513

516

519

519

522

534

537

$519 \quad 537$

$540 \quad 546 \quad 555$

$552 \quad 558 \quad 582$

$510 \quad 540 \quad 540$

$567 \quad 570$

$525 \quad 531$

$597 \quad 600$

$615 \quad 618$

549

552

552

555

558

564

$576 \quad 579$

$480 \quad 573$

480

576

576

585

528

561

480

561

564

567

573

486

537

555 
start

stop

$\mathrm{x}$
510

$513 \quad 543$

$522 \quad 534$

$594 \quad 594$

$600 \quad 606$

$612 \quad 612$
588

594

606

612
$594 \quad 594$

594

606

612
$594 \quad 594$

600

606

606
594

600

606 
633 SUB F step(Opt,Hi_lo_port,Max_pts,Nfreqs, F_Iist(*),Bail\$,Proc)

636 OPTION BASE 1

639 Bail\$="N"

642 MAT F list=ZER

645 Start: DIS̄P "INPUT START,STOP,STEP(GHz) e.g., $2, .1$ ";CHR\$(7);

648

651

INPUT F1, F2, F3

PRINT LIN(2), "FREQUENCY INPUT IS FROM";F1;"TO";F2;"STEP"

654

657

660

663

666

669

672

675

678

681

684

687

690

693

696

699

702

SUBEND ;F3;"GHz" ; CHR\$ (7)

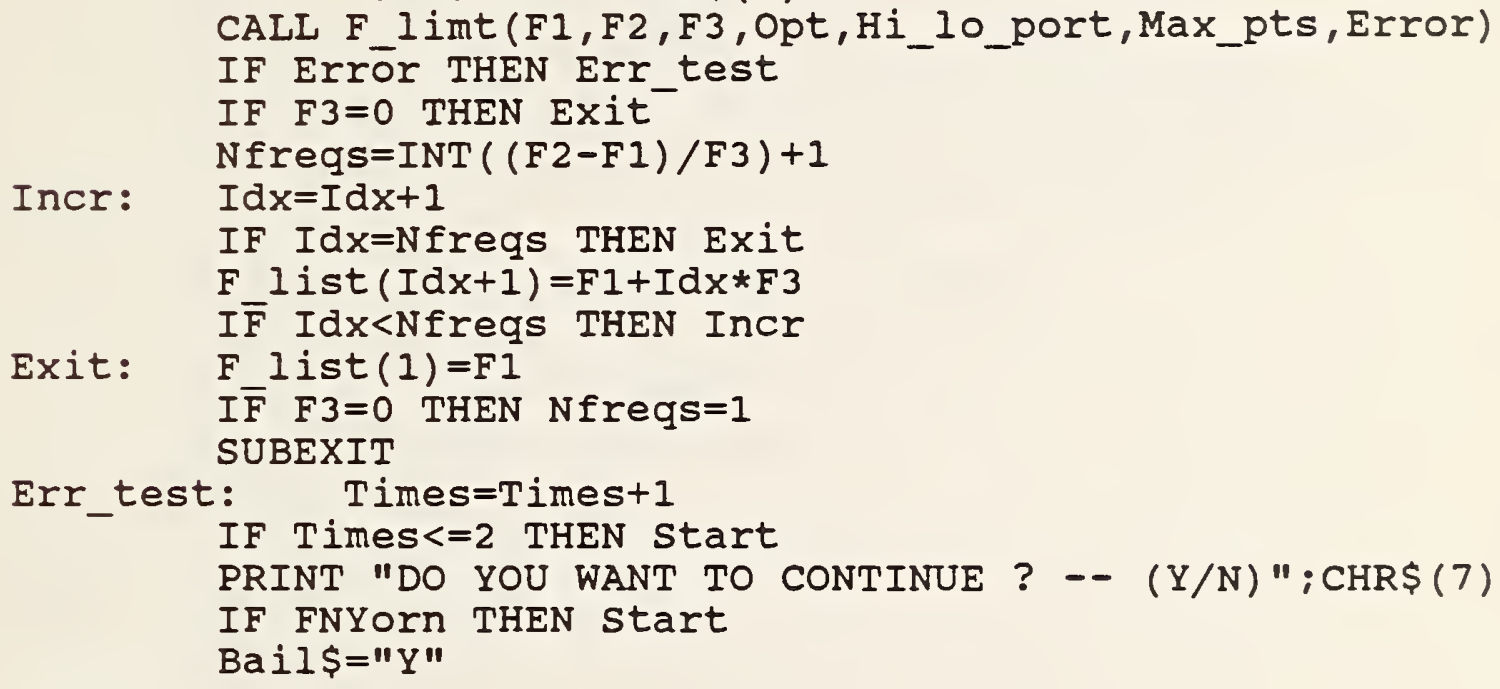

705 ! $* * * * * * * * * * * * * * *$ SUBPROGRAM F_TYPE

708 ! FILE NAME: F_TYPE

! 811216-0900---BCY

S F_step(

\begin{tabular}{|c|c|c|c|c|c|c|c|}
\hline Bail\$ & 633 & 639 & 699 & & & & \\
\hline Err_test: & 687 & 657 & & & & & \\
\hline Error & 654 & 657 & & & & & \\
\hline Exit: & 678 & 660 & 669 & & & & \\
\hline F1 & 648 & 651 & 654 & 663 & 672 & 678 & \\
\hline F2 & 648 & 651 & 654 & 663 & & & \\
\hline F3 & 648 & 651 & 654 & 660 & 663 & 672 & 681 \\
\hline F_list & 633 & 642 & 672 & 678 & & & \\
\hline Hi_lo_port & 633 & 654 & & & & - & \\
\hline$I d x$ & 666 & 666 & 669 & 672 & 672 & 675 & \\
\hline Incr: & 666 & 675 & & & & & \\
\hline Max_pts & 633 & 654 & & & & & \\
\hline
\end{tabular}


Nereqs

opt

Proc

start:

Times
633

663

669

675

654

633

645

687

690

696

687

690 
711 SUB F_type(Opt,Hi_lo_port,Max_pts,Nfreqs,F(*),Bail\$)

714 OPTION BASE 1

717 DIM Linput\$[160]

720 Bail\$="N"

723 MAT F=ZER

726 start: $I=0$

729

732

735

738

741

744

Incre: $\quad I=I+1$

PRINT CHR\$(12)

DISP " "

PRINT "INPUT FREQUENCY LIST -- ANY ORDER"

PRINT LIN(1);"TERMINATE FREQUENCY LIST WITH 0

IF LESS THAN ";Max_pts;" FREQUENCIES";CHR\$(7)

747

750

753

756

759

762

765

768

771

774

777

780

783

786

789

792

795

798

801

804

807

810

813

816

819

822

825

828

831

834

837

840

843

846

849

852

855

858

SUBEXIT

PRINT LIN(1);"LIST TERMINATAES WITH " Max_pts;"

FREQUENCIES INPUT"; $\operatorname{CHR\$ (10);CHR\$ (7)}$

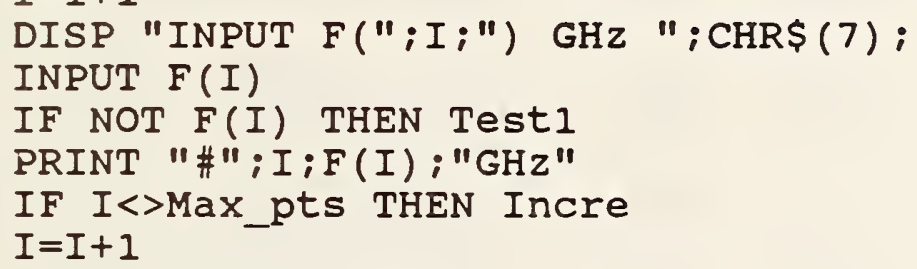

Changes:

DISP "CHANGES ?--(Y/N)";CHRS ( 7);

IF NOT FNYOIn THEN RETURN

PRINT CHR\$(12)

Inc: $\quad I=0$

PRINT "\#"; I;F(I);" GHZ"

List: $\quad$ DISP "FREQUENCY LIST \# ?" ; CHR\$ (7);

LINPUT " " Linput\$

IF NOT FNNumi(Linput\$) THEN List

Digit=VAL (Linput\$)

IF (Digit<=0) OR (Digit $>$ Nfreqs) THEN List

DISP "CORRECTED \# "iDigit;" ";CHRS(7);

INPUT F(Digit)

PRINT LIN(1); "CORRECTED \#:"

GOTO Changes

PRINT SPA (5);"\#";Digit;SPA(2);F(Digit);" GHz";CHR\$(7)

Err_test: Times=Times+1

IF Times $<=2$ THEN RETURN

861

864

SUBEND

PRINT LIN (2);"DO YOU WANT TO CONTINUE ? -- (Y/N)";CHR\$ (7)

IF FNYOIn THEN RETURN

Bail\$="Y"

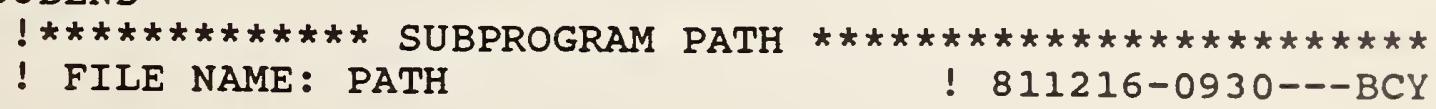


s F_typer

\begin{tabular}{|c|c|c|c|c|c|c|c|}
\hline Bail\$ & 711 & 720 & 855 & & & & \\
\hline Changes: & 792 & 771 & 840 & & & & \\
\hline Chng: & 771 & 786 & & & & & \\
\hline Digit & 822 & 825 & 825 & 828 & 831 & 837 & 837 \\
\hline Err_test: & 843 & 783 & & & & & \\
\hline Error & 780 & 783 & 786 & & & & \\
\hline F( & $\begin{array}{l}711 \\
780\end{array}$ & $\begin{array}{l}723 \\
807\end{array}$ & $\begin{array}{l}750 \\
831\end{array}$ & $\begin{array}{l}753 \\
837\end{array}$ & 756 & 777 & 780 \\
\hline Hi_10_port & 711 & 780 & & & & & \\
\hline$I$ & $\begin{array}{l}726 \\
756 \\
804\end{array}$ & $\begin{array}{l}744 \\
759 \\
804\end{array}$ & $\begin{array}{l}744 \\
762 \\
807\end{array}$ & $\begin{array}{l}747 \\
762 \\
807\end{array}$ & $\begin{array}{l}750 \\
765 \\
810\end{array}$ & $\begin{array}{l}753 \\
768\end{array}$ & $\begin{array}{l}756 \\
801\end{array}$ \\
\hline Inc: & 804 & 810 & & & & & \\
\hline Incre: & 744 & 759 & & & & & \\
\hline Linput\$ & 717 & 816 & 819 & 822 & & & \\
\hline List: & 813 & 819 & 825 & & & & \\
\hline Max_pts & 711 & 738 & 741 & 759 & 780 & & \\
\hline Nfreqs & 711 & 768 & 777 & 780 & 810 & 825 & \\
\hline Opt & 711 & 780 & & & & & \\
\hline start: & 726 & 765 & & & & & \\
\hline Test1: & 765 & 753 & & & & & \\
\hline Times & 843 & 843 & 846 & & & & \\
\hline
\end{tabular}


867 SUB Path(Hi_lo_port, Nfregs, F_list(*), Amps(*), Path(*))

870 OPTION BASE 1

873 Hi_lo=Hi_lo_port+1

876 Inc_í $\bar{I}=I \overline{+}$

879 IF I>Nfregs THEN SUBEXIT

$882 \quad \mathrm{X}=\mathrm{F}$ Iist (I)

885

888

891

894

897

900

903

906

SUBEXIT

ON $\overline{\mathrm{H}} \mathrm{i}$ lo GOSUB LO, $\mathrm{Hi}$

ON Amp GOSUB Amp1, Amp2 , Amp3, Amp4, Amp5

$\operatorname{Path}(I)=$ Path

IF Amp<=Last_amp THEN Inc_i

Amps (Amp) $=A m \bar{p}$

Last_amp=Amp

IF I<Nfregs THEN Inc $i$

909 LO: $\quad A m p=1$

912 IF $X>1$ THEN AMP=2

915 RETURN

918 Hi: $\quad A m p=3$

921

924

927

RETURN

IF $X>4$ THEN $A m p=4$

930

Ampl:

IF $X>7.8$ THEN Amp $=5$

933

936

939

942

945

948

951

954

957

960

963

966

969

972

975

978

Path $=1$

IF $\mathrm{X}>.35$ THEN Path=2

IF $X>.5$ THEN Path=3

RETURN

IF $\mathrm{X}>.7$ THEN Path $=4$

Amp2: $\quad$ Path $=5$

RETURN

IF $\mathrm{X}>1.3$ THEN Path $=6$

Amp3:

Path $=7$

IF $\mathrm{X}>2.5$ THEN $\mathrm{Path}=8$

RETURN

Amp4: $\quad$ Path $=9$

RETURN

IF $\mathrm{X}>5.4$ THEN Path $=10$

Amp5:

Path $=11$

IF $\mathrm{X}>10.5$ THEN Path $=12$

981 SUBEND

984 ! $* * * * * * * * * * * * * *$ SUBPROGRAM F_LIMT
987 ! FILE NAME: F_LIMT

! 811208-1600---BCY

S Path (

Amp

888

918

894

897

924

Amp1:

930

888

Amp2 :

945

888

Amp3 :

954

888

Amp4:

963

888 


\begin{tabular}{|c|c|c|c|c|c|c|c|}
\hline Amp5: & 972 & 888 & & & & & \\
\hline Amps ( & 867 & 897 & & & & & \\
\hline F_list & 867 & 882 & & & & & \\
\hline $\mathrm{Hi}:$ & 918 & 885 & & & & & \\
\hline $\mathrm{Hi}$ & 873 & 885 & & & & & \\
\hline Hi_lo_port & 867 & 873 & & & & & \\
\hline I & 876 & 876 & 879 & 882 & 891 & 903 & \\
\hline Inc_i: & 876 & 894 & 903 & & & & \\
\hline Last_amp & 894 & 900 & & & & & \\
\hline Lo: & 909 & 885 & & & & & \\
\hline Nfreqs & 867 & 879 & 903 & & & & \\
\hline Path & $\begin{array}{l}891 \\
954\end{array}$ & $\begin{array}{l}930 \\
957\end{array}$ & $\begin{array}{l}933 \\
963\end{array}$ & $\begin{array}{l}936 \\
966\end{array}$ & $\begin{array}{l}939 \\
972\end{array}$ & $\begin{array}{l}945 \\
975\end{array}$ & 948 \\
\hline Path ( & 867 & 891 & & & & & \\
\hline $\mathrm{X}$ & $\begin{array}{l}882 \\
948\end{array}$ & $\begin{array}{l}912 \\
957\end{array}$ & $\begin{array}{l}921 \\
966\end{array}$ & $\begin{array}{l}924 \\
975\end{array}$ & 933 & 936 & 939 \\
\hline
\end{tabular}


990 SUB F_limt(F1,F2,F3,Opt,Hi_lo_port,Max_pts,Error)

993 OPTION BASE 1

996 Error $=0$

997 PRINT "HILOPORT";Hi_lo_port

999 IF Opt>1 THEN Test

1002 IF F1>F2 THEN Msg1

1005 IF $F 1+F 3>$ F2 THEN Msg 2

1008 IF $(F 1<>F 2)$ EXOR (F3<>0) THEN Msg6

101 Min_step $=(F 2-F 1) /($ Max_pts-1)

1014 IF $\bar{M}$ in_step>F3 THEN Msg3

1017 Test: -IF Hi_lo_port AND (F2>18) OR NOT Hi_lo_port AND $(\mathrm{F} 2>2 \overline{)} \mathrm{T} \overline{\mathrm{H}} \mathrm{N}$ Msg 4

1020 IF Hi_lo_port AND (FI<2) OR NOT Hi_lo_port AND

\section{SUBEXIT} $(\mathrm{F} 1<. \overline{2} 5)$ THEN MSg5

1026 MSg1: PRINT "START>STOP";CHR\$(7)

1029 GOTO Exit

1032 MSg2: PRINT "STEP TOO LARGE", CHR\$(7)

1035 GOTO Exit

1038 Msg3: IMAGE "MINIMUM STEP TO COVER RANGE REQUESTED IS ", Z.6D," GHZ", K

1041 PRINT USING Msg3;Min_step;CHR\$ (7)

1044 GOTO Exit

1047 Msg4: PRINT "HIGH FREQUENCY OUT OF BOUNDS", CHR\$(7)

1050 GOTO Exit

1053 MSg5: PRINT "LOW FREQUENCY OUT OF BOUNDS", CHR\$(7)

1056 GOTO Exit

1059 MSg6: PRINT "START, STOP, STEP FREQUENCIES ARE INCONSISTENT",

1062 Exit: 'Error=1

1065 SUBEND

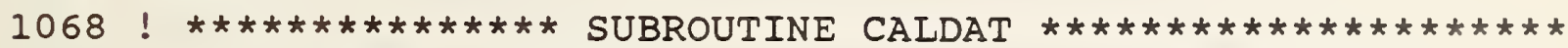

1071 ! FILE NAME : CALDAT

! 810916-2120-- BCY

1074

S F_limt(

\begin{tabular}{|c|c|c|c|c|c|c|c|}
\hline Error & 990 & 996 & 1062 & & & & \\
\hline Exit: & 1062 & 1029 & 1035 & 1044 & 1050 & 1056 & \\
\hline F1 & 990 & 1002 & 1005 & 1008 & 1011 & 1020 & 1020 \\
\hline F2 & 990 & 1002 & 1005 & 1008 & 1011 & 1017 & 1017 \\
\hline F3 & 990 & 1005 & 1008 & 1014 & & & \\
\hline Hi_lo_port & 990 & 997 & 1017 & 1017 & 1020 & 1020 & \\
\hline Max_pts & 990 & 1011 & & & & & \\
\hline Min_step & 1011 & 1014 & 1041 & & & & \\
\hline Msg1: & 1026 & 1002 & & & & & \\
\hline
\end{tabular}


Msg2:

Msg 3 :

Msg4:

Msg5:

Msg6:

opt

Test:
$1032 \quad 1005$

$1038 \quad 1014$

$1047 \quad 1017$

$1053 \quad 1020$

$1059 \quad 1008$

$990 \quad 999$

1017999 
1077 SUB Caldat (Max_pts, Nfreqs, Repeats, Dim,Hi_lo_port, Freq_a(*), Typs, sp, st $\bar{d}$ mnt, Print\$, Path_a (*) ,Line $(* \overline{)}$, Lines, Fstop_a (*) , Cnnct $\$$, Mounts, sn (*), Cal)

1080 OPTION BASE 1

1083 DIM Freq_o(Nfreqs), Path_o(Nfreqs), Fstop_o(1), Type\$[7], Id_content $\$[80]$

1086 DIM Map (Nfreqs), Power(Nfreqs, Repeats, Dim, 10), Rvgl(2), Rvg2 (2) , Pi (Nfreqs, 2 , Repeats, Dim), Pmnt (Nfreqs, Repeats, Dim, 2)

1089 REDIM Freq_a(Nfreqs), Path_a (Nfreqs), Fstop_a (Nfreqs)

1092 PRINTER IS 16

1093 Cal $\$=$ VAL $\$$ (Cal)

1095 CALL Fdata (Nfreqs, Freq_a(*), Freq_o(*), Path_a(*), Path_o(*), Fstop_o(*), Map (*))

1098 TYp flag=POS (TȲp\$, "P")

1101 IF TYp_flag>1 THEN Meas_x! ! ! ! ! ! ! ! ! ! ! ! ! ! ! ! ! ! ! ! ! !

1104

1107

1110

1119 ! - CALIBRATE ALL BUT LINES

End $=1$

Devices $=6$

Type $\$=$ "ORDERED"

CALL Measre (Type\$, Repeats, End, Freq_o(*), Path_o(*), Line (*), Fstop_o(*), Hi_lo_port, $\bar{N}$ freqs, Devices, Rvgl(*), $\operatorname{Rvg} 2(\overline{)})$, Power $(*), \operatorname{Map}(*), \operatorname{Typ} \$, \mathrm{Sp}, \mathrm{Pi}(*), \operatorname{Pmnt}(*)$, $\operatorname{Sn}(*))$

1122

1125

1128

1131

End $=4$

Devices=Lines

Type\$="AIRLINE"

CALL Measre (Type\$, Repeats, End, Freq_a (*), Path_a (*), Line (*), Fstop a (*), Hi 10 port, $\bar{N}$ freqs, Devíces, Rvgl (*), $\operatorname{Rvg} 2(\bar{*})$, Power $(*), \operatorname{Map}(*), \operatorname{Typ}, \operatorname{Sp}, \mathrm{Pi}(*), \operatorname{Pmnt}(*)$, $\operatorname{Sn}(*))$

1134 GOTO Index

1137

! -

1140 Meas_x: End=1+3* (Typ_flag=2) ! ! ! ! ! ! ! ! ! ! ! ! ! ! ! ! ! ! ! !

1143

1146

1149

TYpe\$="ORDERED"

CALL Measre (Type\$, Repeats, End, Freq_o(*), Path_o(*), Line(*), Fstop_o(*), Hi_lo_port, $\bar{N}$ freqs, Devices, Rvgl (*), $\operatorname{Rvg} 2(\bar{*})$, Power $(*), \operatorname{Map}(*), \operatorname{Typ} \$, \operatorname{Sp}, \operatorname{Pi}(*), \operatorname{Pmnt}(*)$, $\operatorname{Sn}(*))$

1152 ! IF TYP_flag=4 THEN DisC

1155 ! IF TYP_flag<>3 THEN Index

1158

1159

IF (TYp_flag=3) AND $(\mathrm{Sp}=3)$ THEN Index

1161

1164

1167

1170

1173

1176

1179

1182

1185

1188 GOTO Index !!!!!!!!!!!!!!!!!!!!!

1191 Incl: $\quad \mathrm{L}=\mathrm{L}+1$ 
Index: $\quad I=0$

$$
\begin{gathered}
\text { Stopk }=16 *(\text { Typ_flag<2) }+4 *(\text { Typ_flag=2) }+(\text { Typ_flag }>2) \\
+(\text { Typ_flag=4)+Mounts }
\end{gathered}
$$

1212 !

1215

1218

1221

1224

1227

1230

1233

1236

1239

1242

1245

1248

1251

1254

1257

1260

1261

1262

1263

1264

1265

1266

1267

1269

1270

1271

1272

1274

1276

1277

1278

1279

1280

1281

1284

1287

1290

1293

1296 SUBEXIT

1299 Typ01: Fileo\$="PWR" \& "H"\&Ca

1302

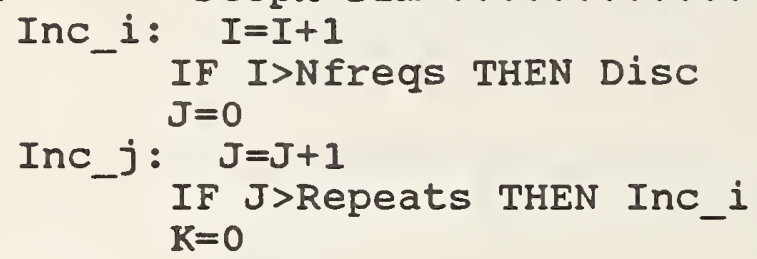




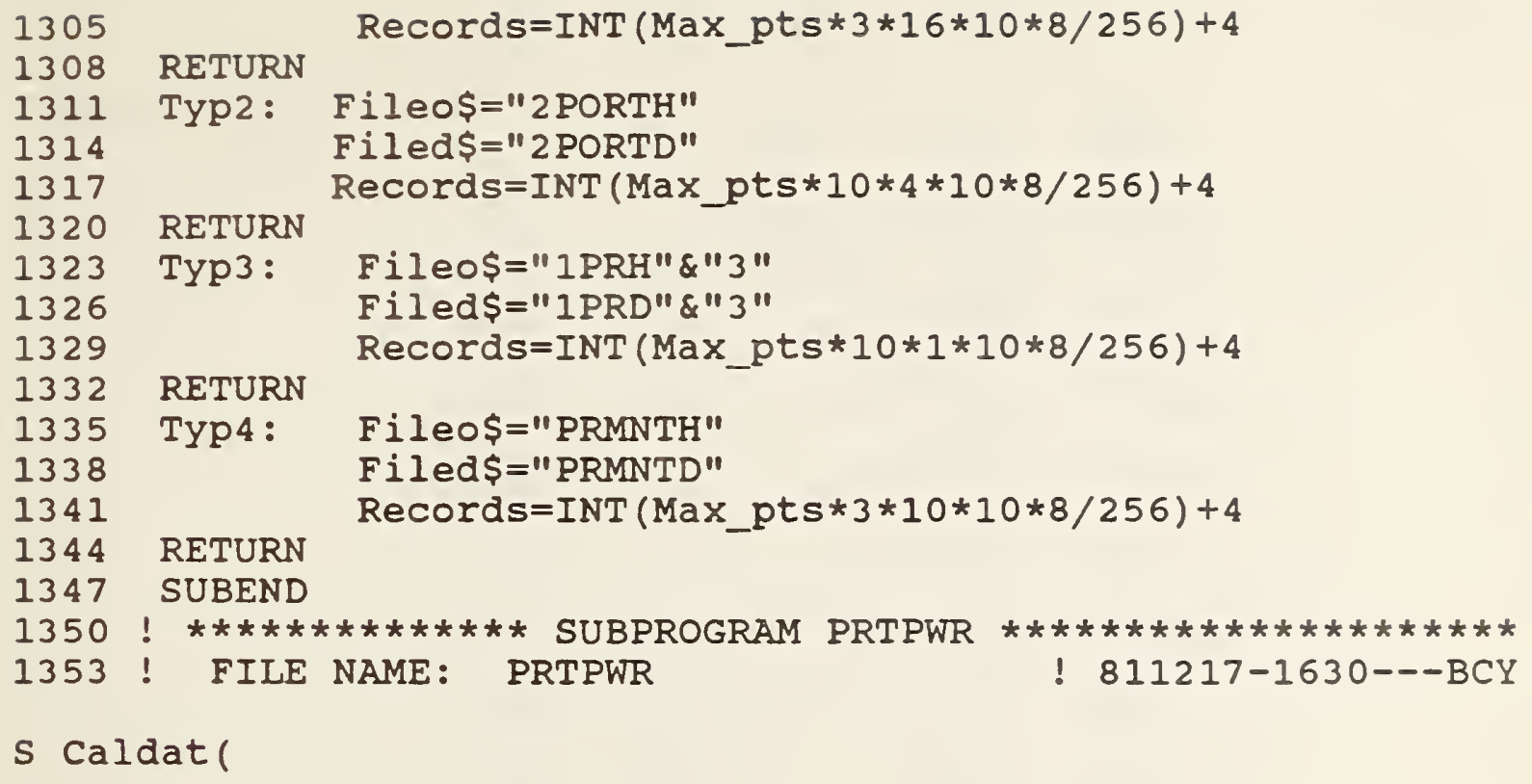

\begin{tabular}{|c|c|c|c|c|c|c|}
\hline Cas & 1263 & 1299 & 1302 & & & \\
\hline $\mathrm{Cal}$ & 1077 & 1093 & 1263 & & & \\
\hline Cal\$ & 1093 & & & & & \\
\hline cnncts & 1077 & 1279 & & & & \\
\hline $\mathrm{Col}$ & 1164 & 1170 & 1197 & & & \\
\hline Date\$ & 1260 & 1279 & 1293 & & & \\
\hline Devices & 1110 & 1116 & 1125 & 1131 & 1143 & 1149 \\
\hline Dim & 1077 & 1086 & 1086 & 1086 & 1293 & \\
\hline Disc: & 1257 & 1218 & & & & \\
\hline Dtg $\$$ & 1257 & 1260 & 1261 & & & \\
\hline End & 1107 & 1116 & 1122 & 1131 & 1140 & 1149 \\
\hline Filed\$ & 1280 & 1302 & 1314 & 1326 & 1338 & \\
\hline Fileo\$ & 1279 & 1299 & 1311 & 1323 & 1335 & \\
\hline Freq_al & 1077 & 1089 & 1095 & 1131 & & \\
\hline Freq_ol & 1083 & 1095 & 1116 & 1149 & 1279 & 1293 \\
\hline Fstop_al & 1077 & 1089 & 1131 & & & \\
\hline Fstop_ol & 1083 & 1095 & 1116 & 1149 & & \\
\hline
\end{tabular}




\begin{tabular}{|c|c|c|c|c|c|c|c|}
\hline Hi_lo_port & 1077 & 1116 & 1131 & 1149 & & & \\
\hline I & $\begin{array}{l}1173 \\
1218\end{array}$ & $\begin{array}{l}1173 \\
1248\end{array}$ & $\begin{array}{l}1176 \\
1248\end{array}$ & 1197 & 1206 & 1215 & 1215 \\
\hline Id_content\$ & 1083 & 1278 & 1279 & & & & \\
\hline Inc_i: & 1215 & 1227 & & & & & \\
\hline Inc_j: & 1224 & 1236 & & & & & \\
\hline Inc_k: & 1233 & 1245 & & & & & \\
\hline Inc_I: & 1242 & 1251 & & & & & \\
\hline Inci: & 1173 & 1185 & & & & & \\
\hline Incj: & 1182 & 1194 & & & & & \\
\hline Incl: & 1191 & 1200 & & & & & \\
\hline Index: & 1206 & 1134 & 1159 & 1176 & & & \\
\hline $\mathrm{J}$ & $\begin{array}{l}1179 \\
1224\end{array}$ & $\begin{array}{l}1182 \\
1227\end{array}$ & $\begin{array}{l}1182 \\
1248\end{array}$ & $\begin{array}{l}1185 \\
1248\end{array}$ & 1197 & 1221 & 1224 \\
\hline $\mathrm{K}$ & $\begin{array}{l}1167 \\
1248\end{array}$ & 1197 & 1230 & 1233 & 1233 & 1236 & 1248 \\
\hline I & $\begin{array}{l}1188 \\
1242\end{array}$ & $\begin{array}{l}1191 \\
1245\end{array}$ & $\begin{array}{l}1191 \\
1248\end{array}$ & $\begin{array}{l}1194 \\
1248\end{array}$ & 1197 & 1239 & 1242 \\
\hline Line( & 1077 & 1116 & 1131 & 1149 & & & \\
\hline Lines & 1077 & 1125 & & & & & \\
\hline $\operatorname{Map}($ & 1086 & 1095 & 1116 & 1131 & 1149 & & \\
\hline Max_pts & 1077 & 1305 & 1317 & 1329 & 1341 & & \\
\hline Meas_x: & 1140 & 1101 & & & & & \\
\hline Mounts & 1077 & 1143 & 1209 & 1293 & & & \\
\hline Nfreqs & $\begin{array}{l}1077 \\
1089 \\
1176\end{array}$ & $\begin{array}{c}1083 \\
1089 \\
1218\end{array}$ & $\begin{array}{c}1083 \\
1089 \\
1279\end{array}$ & $\begin{array}{c}1086 \\
1095 \\
1293\end{array}$ & $\begin{array}{l}1086 \\
1116\end{array}$ & $\begin{array}{l}1086 \\
1131\end{array}$ & $\begin{array}{l}1086 \\
1149\end{array}$ \\
\hline Path_al & 1077 & 1089 & 1095 & 1131 & & & \\
\hline Path_ol & 1083 & 1095 & 1116 & 1149 & & & \\
\hline Pil & 1086 & 1116 & 1131 & 1149 & 1293 & & \\
\hline Pint ( & 1086 & 1116 & 1131 & 1149 & & & \\
\hline
\end{tabular}




\begin{tabular}{|c|c|c|c|c|c|c|c|}
\hline Power( & $\begin{array}{l}1086 \\
1280\end{array}$ & $\begin{array}{l}1116 \\
1293\end{array}$ & 1131 & 1149 & 1197 & 1248 & 1248 \\
\hline Prints & 1077 & 1293 & & & & & \\
\hline Records & 1280 & 1305 & 1317 & 1329 & 1341 & & \\
\hline Repeats & $\begin{array}{l}077 \\
1185\end{array}$ & $\begin{array}{c}1086 \\
1227\end{array}$ & $\begin{array}{r}1086 \\
1279\end{array}$ & $\begin{array}{l}1086 \\
1293\end{array}$ & 1116 & 1131 & 1149 \\
\hline Rvgl( & 1086 & 1116 & 1131 & 1149 & 1293 & & \\
\hline Rvg2 ( & 1086 & 1116 & 1131 & 1149 & 1293 & & \\
\hline $\sin ($ & 1077 & 1116 & 1131 & 1149 & & & \\
\hline $\mathrm{sp}$ & 1077 & 1116 & 1131 & 1149 & 1170 & 1279 & \\
\hline std_mnt & 1077 & 1279 & & & & & \\
\hline stopk & 1209 & 1236 & & & & & \\
\hline Time\$ & 1261 & 1279 & 1293 & & & & \\
\hline Typ\$ & 1077 & 1098 & 1116 & 1131 & 1149 & 1279 & \\
\hline Typ01: & 1299 & 1276 & 1276 & & & & \\
\hline Typ2 : & 1311 & 1276 & & & & & \\
\hline TYp3 : & 1323 & 1276 & & & & & \\
\hline TYp4 : & 1335 & 1276 & & & & & \\
\hline Typ_flag & $\begin{array}{l}1098 \\
1276\end{array}$ & $\begin{array}{l}1101 \\
1293\end{array}$ & 1140 & 1209 & 1209 & 1209 & 1209 \\
\hline Type\$ & 1083 & 1113 & 1116 & 1128 & 1131 & 1146 & 1149 \\
\hline
\end{tabular}


1356 SUB Prtpwr(Print\$,Nfreqs, Repeats, Typ_flag,Rvgavl(*), Rvgav2 (*), Freq_o(*), Power $(*)$, Date\$, Time\$, Pi(*), Mounts, Dim)

1359 OPTION BASE 1

1362 DISP " "

1365

IF POS(Print\$,",") THEN PRINTER IS 7,1

1368

IF POS(Print\$,"O") THEN PRINTER IS 0

1371

PRINT TAB (10); "RVG1 (VOLTS)"; TAB(44);"RVG2 (VOLTS) ";TAB(67); Date\$\&" . "\&Time\$

1374 PRINT USING Headero;Rvgav1 (1) ;Rvgav1 (2) iRvgav2 (1) ;Rvgav2 (2)

1375 PRINT LIN(2) :TAB(29); "SIDE-ARM POWER DATA"

1380 PRINT TAB(33); "MILLIWATTS"

1383 stopk=Dim

1386 Inci: $I=I+1$

1389

1392

1395

1398

1401

1404

1407

1410

1413

1416

1419

1422

1425

1428

1431

1434

1437

1440

1443

1446

1449

1452

1455

1458

1461

1464

1467

1470

1473

1476

1479

1482

1485

1488

1491

1494

1497

IF I>Nfreqs THEN Exit

PRINT USING Headerl;"FREQUENCY" ;Freq_o(I);" GHz" ;Repeats; " REPEAT (S)"

PRINT LIN(1); TAB(19);"6-PORT \#1";TAB(43);CHR\$(124); TAB (59); "6-PORT \#2"; LIN (1)

PRINT USING Header2;"MNT1 ";"MNT2 ";"MNT3 ";"MNT4 "; "MNT1 "; "MNT2 ";"MNT3 ";"MNT4 "

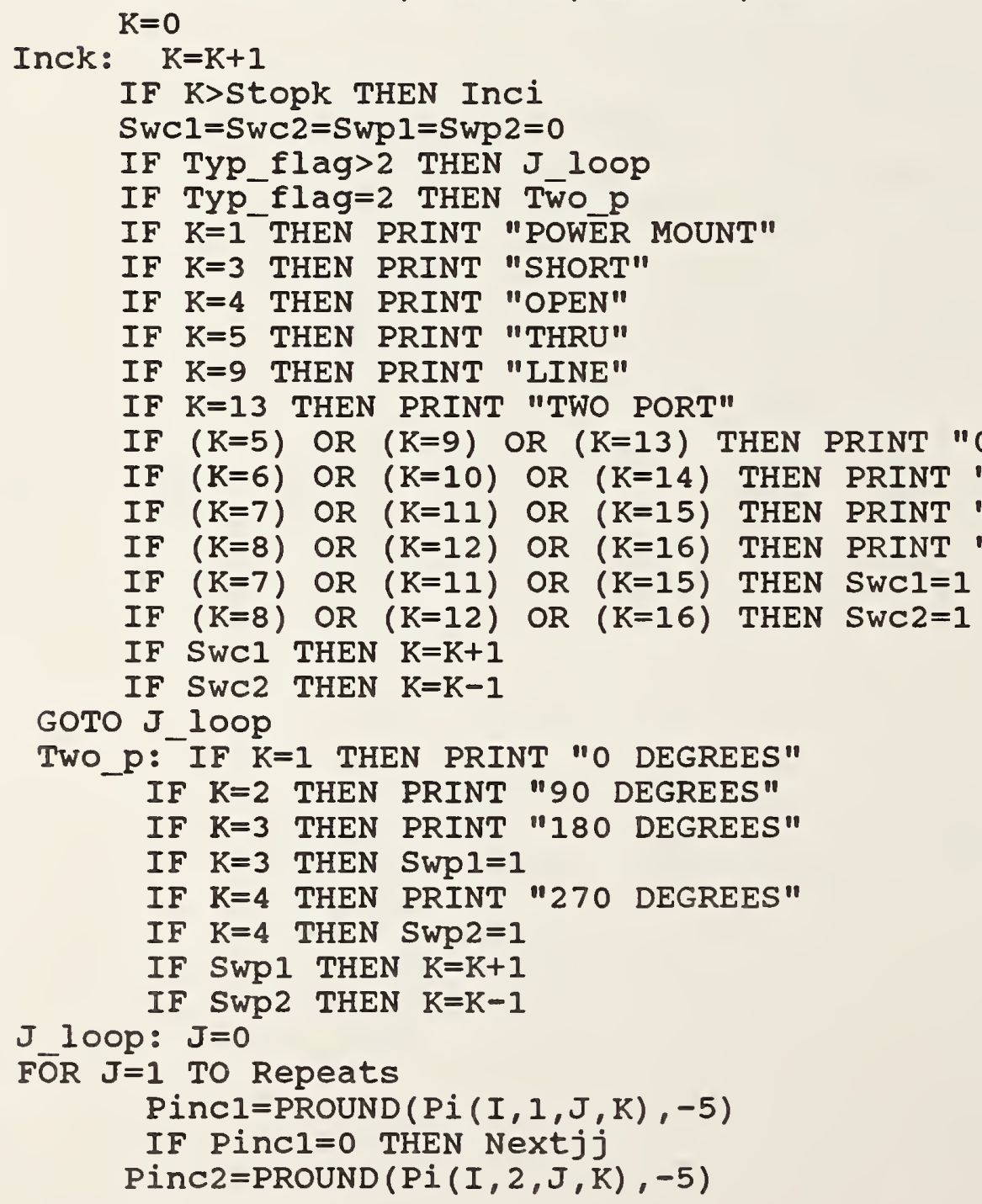


1503 PRINT "Pinc1= "; Pinc1, "Pinc2= "; Pinc2, "Pratio=";

1506 Nextjj: NEXT J PROUND (Pinc2/Pinc1, -5)

$1509 \mathrm{~J}=0$

1512 Incj: $\mathrm{J}=\mathrm{J}+1$

1515

1518

1521

1524

1527

1530

1533

1536

1539

1542

1545

1548

1551

1554

1557

1560

1563

1566

GOTO Inck

IF $J<=$ Repeats THEN I_loop

PRINT

IF SWCl OR SWP1 THEN $\mathrm{K}=\mathrm{K}-1$

IF SWC2 OR SWp2 THEN $\mathrm{K}=\mathrm{K}+1$

L_loop: $\mathrm{L}=0$

Iñcl: $\mathrm{L}=\mathrm{L}+1$

IF L $>8$ THEN PRINT

IF $\mathrm{L}>8$ THEN InCj

GOTO Incl

Header0: IMAGE \#, 2X,D.7D,6X

Header1: IMAGE //9A, 3D.6D, 4A, 5X, 2D, 10A/

Header2: IMAGE $8(5 \mathrm{X}, 5 \mathrm{~A}) /$

Form 1: IMAGE \#, $2 X, 2 \mathrm{D} .5 \mathrm{D}$

Exit: PRINTER IS 16

DISP " "

SUBEND

1569 ! $* * \star \star * * * * * \star *$ SUBPROGRAM FDATA

1572

FILE NAME:

FDATA

! 811217-1400---BCY

S Prtpwr(

Date\$

$1356 \quad 1371$

Dim

$1356 \quad 1383$

Exit:

$1560 \quad 1389$

Form1:

1557

Freq_o(

$1356 \quad 1392$

Headero:

1548

Header 1:

1551

Header2:

1554

I

1386

1386

1389

1392

$1494 \quad 1500$

1542

Inci:

$1386 \quad 1407$

Incj :

$1512 \quad 1539$

Inck:

$1404 \quad 1527$

Incl:

$1533 \quad 1545$ 
$\begin{array}{lllllll}1488 & 1491 & 1494 & 1500 & 1506 & 1509 & 1512\end{array}$

J_100p:

$\begin{array}{lll}1512 & 1515 & 1542\end{array}$

K

$1488 \quad 1413 \quad 1461$

$\begin{array}{lllllll}1401 & 1404 & 1404 & 1407 & 1419 & 1422 & 1425\end{array}$

$\begin{array}{lllllll}1428 & 1431 & 1434 & 1437 & 1437 & 1437 & 1440\end{array}$

$\begin{array}{lllllll}1440 & 1440 & 1443 & 1443 & 1443 & 1446 & 1446\end{array}$

$\begin{array}{lllllll}1446 & 1449 & 1449 & 1449 & 1452 & 1452 & 1452\end{array}$

$\begin{array}{lllllll}1455 & 1455 & 1458 & 1458 & 1464 & 1467 & 1470\end{array}$

$\begin{array}{lllllll}1473 & 1476 & 1479 & 1482 & 1482 & 1485 & 1485\end{array}$

$\begin{array}{lllllll}1494 & 1500 & 1521 & 1521 & 1524 & 1524 & 1542\end{array}$

L

$\begin{array}{llllll}1530 & 1533 & 1533 & 1536 & 1539 & 1542\end{array}$

L_loop:

$1530 \quad 1515$

Mounts

1356

Nextjj:

$1506 \quad 1497$

Nfreqs

$1356 \quad 1389$

Pil

$1356 \quad 1494 \quad 1500$

Pincl

$\begin{array}{llll}1494 & 1497 & 1503 & 1503\end{array}$

Pinc2

$\begin{array}{lll}1500 & 1503 \quad 1503\end{array}$

Power (

$1356 \quad 1542$

Print\$

$\begin{array}{lll}1356 & 1365 \quad 1368\end{array}$

Repeats

$\begin{array}{llll}1356 & 1392 & 1491 & 1515\end{array}$

Rvgavi(

$1356 \quad 1374 \quad 1374$

Rvgav2 (

$1356 \quad 1374 \quad 1374$

stopk

$1383 \quad 1407$

Swc1

$\begin{array}{llll}1410 & 1449 & 1455 & 1521\end{array}$

Swc2

$\begin{array}{llll}1410 & 1452 \quad 1458 \quad 1524\end{array}$

Swpl

$\begin{array}{llll}1410 & 1473 \quad 1482 & 1521\end{array}$

Swp2

$\begin{array}{llll}1410 & 1479 \quad 1485 & 1524\end{array}$

Time\$

$1356 \quad 1371$

Two_p:

1464. 1416

Typ_flag

$1356 \quad 1413 \quad 1416$ 
1575 SUB Fdata(Nfreqs,Freq_a(*), Freq_o(*), Path_a(*), Path_o(*), Fstop_o(*), Map (*))

1578 OPTION BĀSE 1

1581 MAT Fstop_o=(Nfreqs)

1584 MAT Freq $\overline{0}=$ Freq_a

1587 MAT Path_o=Path_a

1590 CALL Ordēer (Freq-o(*), Nfreqs)

1593 CALL Order (Path_o(*),Nfreqs)

1596 Inc_i: $I=I+1$

1599 IF I $>$ Nfregs THEN SUBEXIT

1602

1605

1608

1611

1614

1617

1620

1623 SUBEND InC 1

1623 SUBEND

$1626 ! * \star \star \star \star * \star * \star \star \star * \star$ SUBPROGRAM MEASURE

1629 ! FILE NAME : MEASRE

! 810916-2120---BCY

S Fdatal

$\begin{array}{lllllll}\text { Freq_a } & 1575 & 1584 & 1614 & & \\ \text { Freq_o( } & 1575 & 1584 & 1590 & 1602 & \\ \text { Fstop_o( } & 1575 & 1581 & & & & \\ \text { I } & 1596 & 1596 & 1599 & 1602 & 1617 & \\ \text { Inc_i: } & 1596 & 1611 & 1620 & & & \\ \text { Inc_j: } & 1608 & 1614 & & & & \\ \text { J } & 1605 & 1608 & 1608 & 1611 & 1614 \\ \text { Map( } & 1575 & 1617 & & & & \\ \text { Nfreqs } & 1575 & 1581 & 1590 & 1593 & 1599 \\ \text { Path_a( } & 1575 & 1587 & & & & \\ \text { Path_o( } & 1575 & 1587 & 1593 & & & \\ \text { Temp } & 1602 & 1614 & & & & \end{array}$


1632 SUB Measre(Type\$, Repeats, End, Freq(*), Path(*), Aline(*), Fstop (*), Hi lo port, Nfreqs, Devices, Rvgl (*), Rvg2 (*), Po_out (*), Map (*), Typ\$,Sp, Pi (*), Pmnt (*), $\operatorname{sn}(*))$

1635 OPTION BASE 1

1638

1641

1644

1647

1650

1653

1656

1659

1662

1665

1668

1671

1674

1677

1680

1683

1686

1689

1692

1695

1698

1701

1702

1704

1707

1710

1713

1716

1719

1722

1725

1728

1731

1734

1737

1740

1743

1746

1749

1752

1755

1758

1761

1764

1765

DIM Von(Repeats, 10), Vinc(Repeats, 10)

DIM Cont\$[41], clear\$[12]

$\operatorname{DIM} \operatorname{Voff1}(1,10), \operatorname{Voff2}(1,10), \operatorname{Vioff1}(1,10), \operatorname{Vioff2}(1,10)$, Clir $\$ 12]$

DIM Wait $\$[24], \operatorname{Clr} \$[18], \operatorname{Edit} \$[20], \operatorname{Cler} \$[12], \operatorname{Ec} \$[1], \operatorname{Clar} \$[12]$, Cnt $\$[41]$

$\operatorname{EC} \$=\operatorname{CHR} \$(27)$

Wait $\$="$ - WAIT"

Cont\$=Ec\$\&"\&a3r34C"\&" - PRESS 'CONTINUE' WHEN READY"

Cnt\$=Ec\$\&"\&a3r26C"\&" - PRESS 'CONTINUE' WHEN READY"

Clear\$=Ec\$\&"m"\&Ec\$\&"\&a3r34C"\&Ec\$\&"J"

$C l r \$=E c \$ \& " m " \& E c \$ \& " \& a 3 r 0 C " \& E c \$ \& " J " \& E c \$ \& " \& a 3 r 9 C "$

Cler\$=Ec\$\&"m"\&Ec\$\&"\&a3r34C" \&Ec\$\&"K"

Edit $\$=E c \$ \& " \& a 3 r 16 C " \& E c \$ \& " Q " \& " E D " \& E c \$ \& " R " \& E c \$ \& " B " \& E c \$ \& " B "$ $\& \operatorname{Ec} \& " 1 "$

Clar $\$=E c \$ \& " m " \& E c \$ \& " \& a 3 r 26 C " \& E c \$ \& " J "$

Clir\$=Ec\$\&"m"\&Ec\$\&"\&a3r28C"\&Ec\$\&"J"

Typ_flag=PoS (Typ\$, "P")

Rvg reads $=10$

Vof $\bar{f} \_r d s=$ Pwr_reads $=$ Dvm_rds $=2$

Nphase=End

T_device=Aline (1)

Móde $=0$

CALL Fase_shft ( $4, \mathrm{Hi}$ lo_port)

IF (Type\$<>"ORDERED") ĀND (Nfreqs<>1) THEN Rfout

IF TYPe\$<> "ORDERED" THEN Pause

IF Typ flag=1 THEN GOSUB Device 3

IF Typ flag=2 THEN GOSUB Device6

IF TYp flag=3 THEN GOSUB Device8

IF Typ flag=4 THEN GOSUB Device 9

IF Hi Io port AND (TYp_flag<>4) THEN OUTPUT 710;"5354"

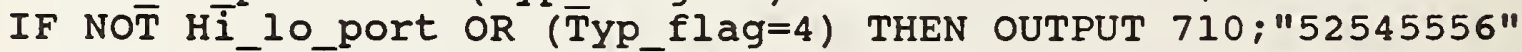

Rfout: CĀLL-Rfout("INIT",Path(*),Freq(*), 1, 1, Hi_lo_port)

IF Type\$<>"ORDERED" THEN Pause

IF (TYp_flag=4) OR NOT Hi_lo_port THEN CALL Rvgs(Rvg_reads, Hi_lo_port, Typ_flag, Rvgl( (*) , $\overline{\operatorname{Rvg} 2(*))}$

$I^{-}$(TYp_flag $<>\overline{4}$ ) AND Hi_lo_port THEN CALL Rvgs(Rvg_reads, Hi_lo_port, Typ_flag, Rvg $\bar{I}(* \overline{)}, \operatorname{Rvg} 2(*))$

PRINT USING "K";EC\$\&"V"

WAIT 3000

IF Typ_flag<>4 THEN PRINT Clear\$;Cont\$;CHR\$(7)

IF Typ_flag=4 THEN PRINT Clear\$;Cnt\$;CHR\$(7)

DISP "-"

Pause: PAUSE

IF TYPE\$="ORDERED" THEN PRINT Clar\$;Edit\$

IF TYpe\$="AIRLINE" THEN PRINT Clear\$;Edit\$

Stop $=0$

Freqs $=1$

IF Type\$<> "AIRLINE" THEN OUTPUT 717, 718; "F3VR1S4T0," 
1766

1767

1770

1773

1776

1779

1782

1785

1788

1791

1794

1797

1800

1803

1806

1809

1812

1815

1818

1821

1824

1827

1830

1833

1836

1839

1842

1845

1848

1851

1854

1857

1860

1863

1866

1869

1872

1875

1878

1881

1884

1887

1890

1893

1896

1902

CALL Fase_shft(1, Hi_lo_port)

FOR Device $=1$ TO Devíces

IF Device $>4$ THEN Nphase $=4$

IF Typ flag=2 THEN Nphase $=4$

IF TYpe $<>$ "AIRLINE" THEN Order

start $=s$ top +1

GOTO Begin

order: start $=1$

Stop=Nfreqs

Begin: Path=Path (start)

OUTPUT 710 USING "4(K/) "; "71"; "41"; "4";"7"

CALL Preads(Voff_rds,Hi_lo_port, 1,Voffl(*), Vioffl(*))

FOR Phase=1 TO Nphase

Cntphi=Cntphi+1

IF (Cntphi=1) AND (Typ_flag=1) THEN DeV

IF (Cntphi=2) AND (Typ_flag=0) THEN cnnct

IF (Cntphi=2) AND (TYp_flag<=1) THEN Dev

IF Typ flag>l THEN Freqs

IF Cntphi<>9 THEN Freqs

IF TYpe\$<> "AIRLINE" THEN Cntphi=13

\section{-}

Freqs: FOR Freqs=start TO stop

Voff: $\quad$ OUTPUT 710 USING "4(K/) ";"71";"41";"4";"7"

CALL Preads(Voff_rds,Hi_lo_port, 1,Voffl(*), Vioffl(*))

IF Typ_flag=4 THEN CALL Pwrmnt(Rvḡ1(*), Rvg2(*), Repeats, Freqs, Device, Pwr reads, Pmnt (*))

IF Type flag $<>4$ THEN OUTPUT 710 USING "4(K/) ";"71";"40"; "4";"7"

Repeat: $\quad$ FOR Repeat=1 TO Repeats

CALL Preads (Dvm_rds,Hi_lo_port, Repeat,Von(*),Vinc(*))

NEXT Repeat

OUTPUT 710 USING "4(K/) "; "71"; $41 " ; " 4 " ; " 7 "$

CALL Preads(Voff_rds,Hi_lo_port, 1,Voff2(*), Vioff2(*))

IF Nfreqs $=1$ THEN PcaIc

IF Freqs<>stop THEN CALL Rfout("NEXT", Path(*), Freq (*), Freqs, start, Hi_lo_port)

If Freqs $<>$ Stop THEN Pcalc

IF (Freqs=Nfreqs) OR (Phase<>4) THEN CALL Rfout

("FIRST" , Path(*), Freq(*), Freqs, Start,Hi_lo_port)

IF (Freqs=Nfreqs) OR (Phase<>4) THEN Pcalc

CALL Rfout("NEXT", Path(*),Freq(*), Freqs, start,Hi_lo_port)

Pcalc: !

CALL Pcalc(Type\$, Typ\$,Hi lo_port, Repeats, Cntphi, Freqs, Rvgl(*), $\operatorname{Rvg} 2(*), \operatorname{Voff1}(*), \operatorname{Vof} 2 \bar{C}^{(*)}, \operatorname{Von}(*), \operatorname{Vinc}(*), \operatorname{Po} \operatorname{out}(*), \operatorname{Map}(*)$, $\operatorname{Pi}(*), \operatorname{Vioffl}(*), \operatorname{Vioff} 2(*))$

\section{NEXT Fregs}

IF (Device>4) OR (TYpe\$="AIRLINE") OR (TYP\$="2PORT")

AND (Fregs-1=Stop) THEN CALL Fase shft (Phase MOD $4+1, \mathrm{Hi}$ lo port)

1899 ! FOR I=start TO stop 
1932

1935

1938

1941

1944

1947

1950

1953

1956

1959

1962

1965

1968

1971

1974

1977

1980

1983

1986

1989

1992

1995

1998

2001

2004

2007

2010

2013

2016

2019

2022

2025

2028

2031

2034

2037

2040

2043

2046

2049

2052

2055

2058
FOR $\mathrm{J}=1$ TO Repeats

IF Type\$<>"ORDERED" THEN Aline

PRINT "Pincl = "; PROUND (Pi ( I, 1, J, Cntph $i),-5)$, "Pinc2= "; PROUND (Pi ( I, 2, J, Cntphi), -5) , "Pratio= "; PROUND (Pi ( I, $2, J$, GOTO Nxxtj Cntphi)/Pi(I, 1,J, Cntphi), -5)

Aline: Pincl=Pi (Map (I) , I,J, Cntphi)

Pinc2 $=P i(\operatorname{Map}(I), 2, J$, Cntph $i)$

PRINT "Pinc1 = "; PROUND (Pinc1, -5), "Pinc2= "; PROUND $($ Pinc2, -5$)$,

NXXtj: NEXT J

"Pratio= "; PROUND (Pinc2/Pinc1, -5)

GOTO Nxtk

FOR $\mathrm{K}=1$ TO 10

IF TYpe\$<>"ORDERED" THEN NXtprt

PRINT USING Form;Po_out( $I, J$, Cntphi, K)

Nxtprt: PRINT USING Form;Po_out(Map(I), J,Cntphi,K)

NXtk: NEXT K

NEXT J

PRINT

PRINT

NEXT I

NEXT Phase

IF TYP_flag=1 THEN Test

IF (Typ_flag<>4) AND (Device=1) THEN Test

IF Device=Devices THEN Dev

GOSUB Device2

PAUSE

PRINT Clir\$;Edit\$

Test: IF Typ_flag>1 THEN Dev

IF NOT TYP flag AND (Device=1) THEN DeV

cnnct:IF Type $<>$ "AIRLINE" THEN GOSUB Connect

PRINT

IF TYpe\$<> AIRLINE" THEN GOTO Paws

IF stop $=$ Nfregs THEN Paws

Nxt device $=$ Aline $($ Stop+1)

IF $\overline{\mathrm{T}}$ device<>Nxt_device THEN GOSUB Connect

PRINT

T_device=Nxt_device

Paws: I $\bar{F}$ Device $=$ Devices THEN Dev

IF TYp flag<>4 THEN PRINT Clear\$;Cont $\$$; CHR $\$$ (7)

IF Typ_flag=4 THEN PRINT Clear\$;Cnt\$;CHR\$(7)

PAUSE

IF TYP_flag<>4 THEN PRINT cler\$;Edit\$

Dev:NEXT Device

IF TYP flag=4 THEN PRINT Clar\$iEdit\$

(- - - - - - - - END MEASUREMENT LOOP

IF TYPE\$<> "ORDERED" THEN Exit

IF NOT ((TYP_flag=0) OR (TYP_flag=4)) THEN what

FOR Freqs $=1$ TO Nfreqs

FOR Reps $=1$ TO Repeats

FOR Device=1 TO Devices

FOR Mnt=1 TO 2

Po_out (Freqs, Reps, Device, $4+$ Mnt $)=$ Pmnt (Freqs, Reps, Device, Mnt ) 
2148 STOP

2151

2154

2157 RETURN

2160 Which:

Rep:
NEXT Mnt

GOTO Rep

Sp2 :

NEXT I

FOR $I=7$ TO 10

Po out (Freqs, Reps, Device, I) $=0$

\section{NEXT Freqs}

Po_out (Freqs, Reps, 1, 1) =Po_out (Freqs, Reps, 1, 6)

Po out (Freqs, Reps, 1, 2) =Pmnt (Freqs, Reps)

FOR $\bar{I}=3$ TO 5

NEXT $\bar{I}$

Po out (Freqs, Reps, 1, I) $=0$

What: IF TYp flag>l THEN Exit

Exit: PRINT EC\$\&"m"; CHR\$ (12)

LOCAL 717,718

RESET 717,718

LOCAL 728

OUTPUT $710 ; " \mathrm{Cl}$

PRINT CHR\$(12)

DISP " "

SUBEXIT

\section{- - CONNECT DEVICES SUBROUTINES \\ Connect: DISP "CONNECT NEXT DEVICE ? -- (Y/N)";CHR\$(7) DO=FNYOIn \\ IF DO THEN $T$ airlin \\ IF NOT DO THEN PRINT EC\$\&"m";CHR\$(12); $\operatorname{LIN}(10) ; \operatorname{TAB}(10) ; "$ PROGRAM HALT -- PRESS RUN TO RESTART"}

T_airlin: IF Type\$ $\bigcirc$ "AIRLINE" THEN Which PRINT USING "\#, $\mathrm{K}^{n} ; \mathrm{Cl}$ r\$;"CONNECT ";Aline(Stop+1);

" CM AIR LINE"; Cont\$; CHR\$ (7)

ON Device+1 GOTO Devicel,Device2, Device3, Device4, Device5,Device6,Device72163 Devicel: PRINT "NOTHING"

2166 Device2:PRINT CHR\$(12);C1r\$;CHR\$(7); "CONNECT MOUNT";

Sn(Device+1); "TO B1 AND 6-PORT 1";CHRS(13)

$\& \operatorname{CHR} \$(10)$; TAB (10); "MOUNT"; Sn(Device+2);"TO B2"

2169

PRINT LIN(1);TAB(10); "IF MOUNT = 0, USE ANY MOUNT";

$\operatorname{LIN}(2) ; \operatorname{TAB}(10) ; "$

PRESS 'CONT' WHEN READY";CHRS(7);

2172 RETURN

2175 Device3: IF Typ_flag THEN PRINT CHR\$(12);Clr\$;CHR\$(7); "CONNECT SHORTS";Wait\$

2178 IF NOT Typ_flag THEN PRINT CHR\$(12);CIr\$;CHR\$(7); "CONNECT SḦORTS"; Cont\$

2181 RETURN

2184 Device4: PRINT USING "\#,K";C1r\$;"CONNECT OPENS";Cont\$;CHR\$(7)

2187

RETURN

2190 Device5: PRINT USING "\#,K";Clr\$;"CONNECT THRU";Cont\$;CHR\$(7)

2193 RETURN

2196 Device6: PRINT USING "\#,K";CIr\$;CHR\$(7) 
2205 RETURN

2208 Device7: PRINT USING "\#,K";Clr\$;"CONNECT ";Aline(1);" CM AIR LINE"; Cont\$;CHR\$(7)

2211 RETURN

2214 Device8 PRINT USING "\#,K";Clr\$;"CONNECT 1-PORT(S)";Wait\$; $\operatorname{HRS}(7)$

2217 RETURN

2220 Device9:PRINT CHR\$(12);C1r\$;CHR\$(7); "CONNECT STD MOUNT";Sn(1); "TO B1 AND 6-PORT 1"; $\operatorname{CHR} \$(13) \& C H R \$(10) ; \operatorname{TAB}(10)$;

2223 RETURN "MOUNT WITH SERIAL NUMBER"; $\operatorname{Sn}(2) ; " T O \quad B 2 "$;Wait\$

2226 Form: IMAGE \#,10(D.5D,X)

2229 SUBEND

2232 ! *************** SUBPROGRAM RVGS $* * * * * * * * * * * * * * * * * * * * * *$ 2235 ! FILE NAME: RVGS ! 810526-1455---BCY

S Measre(

\begin{tabular}{|c|c|c|c|c|c|c|c|}
\hline Aline( & 1632 & 1692 & 2004 & 2154 & 2208 & & \\
\hline Aline: & 1917 & 1908 & & & & & \\
\hline Begin: & 1797 & 1788 & & & & & \\
\hline Clar\$ & 1647 & 1674 & 1752 & 2031 & & & \\
\hline Clear\$ & 1641 & 1662 & 1740 & 1743 & 1755 & 2019 & 2022 \\
\hline Cler\$ & 1647 & 1668 & 2028 & & & & \\
\hline Clir\$ & 1644 & 1677 & 1983 & & & & \\
\hline C1r\$ & $\begin{array}{l}1647 \\
2190\end{array}$ & $\begin{array}{l}1665 \\
2196\end{array}$ & $\begin{array}{l}2154 \\
2208\end{array}$ & $\begin{array}{l}2166 \\
2214\end{array}$ & $\begin{array}{l}2175 \\
2220\end{array}$ & 2178 & 2184 \\
\hline Cnnct: & 1992 & 1818 & & & & & \\
\hline CntS & 1647 & 1659 & 1743 & 2022 & & & \\
\hline Cntphi & $\begin{array}{l}1785 \\
1830 \\
1920\end{array}$ & $\begin{array}{l}1812 \\
1890 \\
1938\end{array}$ & $\begin{array}{l}1812 \\
1911 \\
1944\end{array}$ & $\begin{array}{l}1815 \\
1911\end{array}$ & $\begin{array}{l}1818 \\
1911\end{array}$ & $\begin{array}{l}1821 \\
1911\end{array}$ & $\begin{array}{l}1827 \\
1917\end{array}$ \\
\hline Connect: & 2136 & 1992 & 2007 & & & & \\
\hline Cont\$ & $\begin{array}{l}1641 \\
2190\end{array}$ & $\begin{array}{l}1656 \\
2199\end{array}$ & $\begin{array}{l}1740 \\
2208\end{array}$ & 2019 & 2154 & 2178 & 2184 \\
\hline DO & 2139 & 2142 & 2145 & & & & \\
\hline Dev: & 2034 & 1815 & 1821 & 1974 & 1986 & 1989 & 2016 \\
\hline
\end{tabular}




\begin{tabular}{|c|c|c|c|c|c|c|c|}
\hline Device & $\begin{array}{l}1767 \\
2016 \\
2160\end{array}$ & $\begin{array}{l}1770 \\
2034 \\
2166\end{array}$ & $\begin{array}{l}1845 \\
2052 \\
2166\end{array}$ & $\begin{array}{l}1896 \\
2058\end{array}$ & $\begin{array}{l}1971 \\
2058\end{array}$ & $\begin{array}{l}1974 \\
2067\end{array}$ & $\begin{array}{l}1989 \\
2073\end{array}$ \\
\hline Devicel: & 2163 & 2160 & & & & & \\
\hline Device2: & 2166 & 1977 & 2160 & & & & \\
\hline Device 3: & 2175 & 1704 & 2160 & & & & \\
\hline Device4: & 2184 & 2160 & & & & & \\
\hline Device5: & 2190 & 2160 & & & & & \\
\hline Device6: & 2196 & 1707 & 2160 & & & & \\
\hline Device7: & 2208 & 2160 & & & & & \\
\hline Device 8: & 2214 & 1710 & & & & & \\
\hline Device9: & 2220 & 1713 & & & & & \\
\hline Devices & 1632 & 1767 & 1974 & 2016 & 2052 & & \\
\hline Dvm_rds & 1686 & 1854 & & & & & \\
\hline $\operatorname{Ec} \$$ & $\begin{array}{l}1647 \\
1665 \\
1671 \\
1674 \\
2145\end{array}$ & $\begin{array}{l}1650 \\
1665 \\
1671 \\
1674\end{array}$ & $\begin{array}{l}1656 \\
1665 \\
1671 \\
1677\end{array}$ & $\begin{array}{l}1659 \\
1665 \\
1671 \\
1677\end{array}$ & $\begin{array}{l}1662 \\
1668 \\
1671 \\
1677\end{array}$ & $\begin{array}{l}1662 \\
1668 \\
1671 \\
1734\end{array}$ & $\begin{array}{l}1662 \\
1668 \\
1674 \\
2106\end{array}$ \\
\hline Edit\$ & 1647 & 1671 & 1752 & 1755 & 1983 & 2028 & 2031 \\
\hline End & 1632 & 1689 & & & & & \\
\hline Exit: & 2106 & 2040 & 2100 & & & & \\
\hline Form: & 2226 & & & & & & \\
\hline Freq( & 1632 & 1722 & 1869 & 1875 & 1881 & & \\
\hline Freqs & $\begin{array}{l}1761 \\
1875 \\
2046 \\
2082\end{array}$ & $\begin{array}{l}1782 \\
1875 \\
2058 \\
2088\end{array}$ & $\begin{array}{l}1836 \\
1878 \\
2058 \\
2097\end{array}$ & $\begin{array}{l}1845 \\
1881 \\
2067\end{array}$ & $\begin{array}{l}1869 \\
1890 \\
2079\end{array}$ & $\begin{array}{l}1869 \\
1893 \\
2079\end{array}$ & $\begin{array}{l}1872 \\
1896 \\
2082\end{array}$ \\
\hline Freqs: & 1836 & 1824 & 1827 & & & & \\
\hline Fstop ( & 1632 & 1782 & & & & & \\
\hline Hi_10_port & $\begin{array}{l}1632 \\
1731 \\
1875\end{array}$ & $\begin{array}{l}1698 \\
1731 \\
1881\end{array}$ & $\begin{array}{l}1716 \\
1766 \\
1890\end{array}$ & $\begin{array}{l}1719 \\
1842 \\
1896\end{array}$ & $\begin{array}{l}1722 \\
1854\end{array}$ & $\begin{array}{l}1728 \\
1863\end{array}$ & $\begin{array}{l}1728 \\
1869\end{array}$ \\
\hline
\end{tabular}


$\begin{array}{lllllll}1902 & 1911 & 1911 & 1911 & 1911 & 1917 & 1920\end{array}$

$\begin{array}{lllllll}1938 & 1944 & 1959 & 2064 & 2067 & 2070 & 2085\end{array}$

$2088 \quad 2091$

$\begin{array}{lllllll}1905 & 1911 & 1911 & 1911 & 1911 & 1917 & 1920\end{array}$

$\begin{array}{lllll}1926 & 1929 & 1938 & 1944 & 1950\end{array}$

K

$\begin{array}{llll}1932 & 1938 & 1944 & 1947\end{array}$

$\operatorname{Map}($

$\begin{array}{lllll}1632 & 1890 & 1917 & 1920 & 1944\end{array}$

Mnt

$2055 \quad 2058 \quad 2058 \quad 2061$

Mode

1695

Nereqs

1632

1701

1794

1866

1875

$1878 \quad 2001$

2046

$\begin{array}{llll}1689 & 1770 & 1773 & 1809\end{array}$

Nxt_device

$20042007 \quad 2013$

Nxtk:

$1947 \quad 1941$

Nxtprt:

$1944 \quad 1935$

Nxxtj:

$1926 \quad 1914$

Order:

$1791 \quad 1776$

Path

1797

Pathe

1632

1722

1797

1869

$1875 \quad 1881$

Pause:

$\begin{array}{lll}1749 & 1702 \quad 1725\end{array}$

Paws :

$2016 \quad 1998 \quad 2001$

Pcalc:

$\begin{array}{llll}1887 & 1866 & 1872 & 1878\end{array}$

Phase

$\begin{array}{lllll}1809 & 1875 \quad 1878 & 1896 & 1962\end{array}$

Pil

$\begin{array}{lllllll}1632 & 1890 & 1911 & 1911 & 1911 & 1911 & 1917\end{array}$

1920

Pinc 1

$1917 \quad 1923 \quad 1923$

Pinc2

$1920 \quad 1923 \quad 1923$

Pmnt(

$\begin{array}{llll}1632 & 1845 \quad 2058 \quad 2082\end{array}$

Po_out(

$\begin{array}{lllllll}1632 & 1890 & 1938 & 1944 & 2058 & 2067 & 2079\end{array}$

$20792082 \quad 2088$

Pwr_reads

$1686 \quad 1845$ 


\begin{tabular}{|c|c|c|c|c|c|c|c|}
\hline Rep: & 2094 & 2076 & & & & & \\
\hline Repeat & 1851 & 1854 & 1857 & & & & \\
\hline Repeat: & 1851 & & & & & & \\
\hline Repeats & $\begin{array}{l}1632 \\
1929 \\
2079\end{array}$ & $\begin{array}{l}1638 \\
2049 \\
2082\end{array}$ & $\begin{array}{l}1638 \\
2049 \\
2082\end{array}$ & $\begin{array}{l}1845 \\
2058 \\
2088\end{array}$ & $\begin{array}{l}1851 \\
2058 \\
2094\end{array}$ & $\begin{array}{l}1890 \\
2067\end{array}$ & $\begin{array}{l}1905 \\
2079\end{array}$ \\
\hline Rfout: & 1722 & 1701 & & & & & \\
\hline Rvg1( & 1632 & 1728 & 1731 & 1845 & 1890 & & \\
\hline Rvg2( & 1632 & 1728 & 1731 & 1845 & 1890 & & \\
\hline Rvg_reads & 1683 & 1728 & 1731 & & & & \\
\hline $\sin ($ & 1632 & 2166 & 2166 & 2220 & 2220 & & \\
\hline $\mathrm{Sp}$ & 1632 & & & & & & \\
\hline Sp2: & 2079 & & & & & & \\
\hline Start & $\begin{array}{l}1779 \\
1881\end{array}$ & $\begin{array}{l}1782 \\
1902\end{array}$ & 1791 & 1797 & 1836 & 1869 & 1875 \\
\hline Stop & $\begin{array}{l}1758 \\
1896\end{array}$ & $\begin{array}{l}1779 \\
1902\end{array}$ & $\begin{array}{l}1782 \\
2001\end{array}$ & $\begin{array}{l}1794 \\
2004\end{array}$ & $\begin{array}{l}1836 \\
2154\end{array}$ & 1869 & 1872 \\
\hline T_airlin: & 2151 & 2142 & & & & & \\
\hline T_device & 1692 & 2007 & 2013 & & & & \\
\hline Test: & 1986 & 1968 & 1971 & & & & \\
\hline Typ\$ & 1632 & 1680 & 1890 & 1896 & & & \\
\hline Typ_flag & $\begin{array}{l}1680 \\
1728 \\
1815 \\
1986 \\
2100\end{array}$ & $\begin{array}{l}1704 \\
1728 \\
1818 \\
1989 \\
2175\end{array}$ & $\begin{array}{l}1707 \\
1731 \\
1821 \\
2019 \\
2178\end{array}$ & $\begin{array}{l}1710 \\
1731 \\
1824 \\
2022 \\
2199\end{array}$ & $\begin{array}{l}1713 \\
1740 \\
1845 \\
2028 \\
2202\end{array}$ & $\begin{array}{l}1716 \\
1743 \\
1968 \\
2031\end{array}$ & $\begin{array}{l}1719 \\
1773 \\
1971 \\
2043\end{array}$ \\
\hline Types & $\begin{array}{l}1632 \\
1776 \\
1998\end{array}$ & $\begin{array}{l}1701 \\
1830 \\
2040\end{array}$ & $\begin{array}{l}1702 \\
1890 \\
2103\end{array}$ & $\begin{array}{l}1725 \\
1896 \\
2151\end{array}$ & $\begin{array}{l}1752 \\
1908\end{array}$ & $\begin{array}{l}1755 \\
1935\end{array}$ & $\begin{array}{l}1765 \\
1992\end{array}$ \\
\hline Type_flag & 1848 & & & & & & \\
\hline Vince & 1638 & 1854 & 1890 & & & & \\
\hline Vioffl( & 1644 & 1842 & 1890 & & & & \\
\hline
\end{tabular}




$\begin{array}{lllllll}\text { Vioff2( } & 1644 & 1863 & 1890 & & \\ \text { Voffl( } & 1644 & 1842 & 1890 & \\ \text { Voff2( } & 1644 & 1863 & 1890 & \\ \text { Voff: } & 1839 & & & & & \\ \text { Voff_rds } & 1686 & 1842 & 1863 & & & \\ \text { Von( } & 1638 & 1854 & 1890 & & \\ \text { Wait\$ } & 1647 & 1653 & 2175 & 2202 & 2214 & 220 \\ \text { What: } & 2100 & 2043 & & & & \\ \text { Which: } & 2160 & 2151 & & & \end{array}$


2238 SUB Rvgs(Reads,Hi_lo_port, Typ_flag,Rvgav1(*), Rvgav2(*))

2241 OPTION BASE 1

2244 DIM Rvg1\$(Reads+1), Rvg2\$(Reads+1), Temp1 (Reads), Temp2(Reads)

2247 Start: Dvm $1=717$

$2250 \quad$ Dvm2 $=718$

2253 Stopj $=1$

2256 IF NOT Hi_lo_port OR (Typ_flag=4) THEN OUTPUT 710;"5556"

2259 IF NOT Hi_lo_port OR (Typ_flag=4) THEN J=1

2262 IF NOT Hi_lo_port OR (Typ_flag=4) THEN Stopj=2

2265 IF NOT Hi_lo_port OR (Typ_flag=4) THEN Inc=1

2268 OUTPUT Dvm1, Dvm2 USING Initial

2271 OUTPUT 709;"0919"

2274 Incj: $\mathrm{J}=\mathrm{J}+1$

2277 IF J>S topj THEN Exit

2280 CALL Dvms (Reads, Rvg1\$(*), Rvg2\$(*))

$2283 \quad I=0$

2286 Inci: $I=I+1$

2289 IF I>Reads THEN Test

GOTO Inci

$\operatorname{Temp} 2(I)=-\operatorname{VAL}(\operatorname{Rvg} 2 \$(I+1))$

2301 Test: Rvgtest=Rvgav1(J)=SUM(Temp1)/Reads

2304 GOSUB Swtch

Maxs $=$ Maxt $=0$

IF NOT Hi_lo_port THEN GOSUB Swtch

2316 Mins $=$ Mint $=9$

$2319 \quad I=0$

2322 Inc i: $I=I+1$

2325

2328

2331

2334

2337

2340

2343

2346

2349

2352

2355
2358

2361

2364

IF I>Reads-I THEN Var

$\operatorname{Max} 1=\operatorname{MAX}(\operatorname{Temp} 1(I), \operatorname{Temp} 1(I+1)$ )

Minl=MIN (Templ (I), Templ (I+1))

$\operatorname{Max} 2=\operatorname{MAX}(\operatorname{Temp} 2(I), \operatorname{Temp} 2(I+1))$

$\operatorname{Min} 2=\operatorname{MIN}(\operatorname{Temp} 2(I), \operatorname{Temp} 2(I+1))$

IF Max $1>$ Maxs THEN Maxs=Max 1

IF Minl<Mins THEN Mins=Minl

IF Max2>Maxt THEN Maxt $=\operatorname{Max} 2$

IF Min2<Mint THEN Mint=Min2

GOTO Inc $i$

Var: Var $1=\bar{M}$ axs-Mins

Var2=Maxt-Mint

PRINT USING "K";CHR\$ (27)\&"\&a15R"

Secline: PRINT "MAXIMUM VARIATION WITH ";Reads;

"READINGS ON RVG(";J+Inc;");"=" $\operatorname{Var} 1 ; \operatorname{LIN}(1)$;

$\operatorname{TAB}(41) ; " R V G(" ; \mathrm{J}+\mathrm{Inc}+1 ; ")=$ ";Var $2 ; "$ VOLTS"

2370 ! IF NOT Hi_1o_port OR (Hi_1o_port=2) THEN OUTPUT 710;"5556"

2373 GOTO Incj

2376 Swtch: IF Rvgtest<2 THEN DISP "RVG MECHANICAL SWITCH(ES)

ARE IN THE WRONG POSITION OR SCANNER IS OFF - - PAUSE";

CHR\$ (7)

IF Rvgtest>2 THEN RETURN 
2397 Exit: OUTPUT 709; "C"

2400 LOCAL Dvm1, Dvm2

2403 RESET Dvm1, Dvm2

2406 IF Hi_lo_port AND (Typ_flag 44) THEN OUTPUT 710;"5,5354" 2409 Initial:IMAGE "F3VR1S7T0,"

2412 SUBEND

2415 ! $* * * * * * * * * * * * * * * *$ SUBPROGRAM DVMS $* * * * * * * * * * * * * * * * * * * * *$ $2418 !$ FILE NAME: DVMS ! 810514-1805-.-BCY

S Rvgs (

\begin{tabular}{|c|c|c|c|c|c|c|c|}
\hline Dvm1 & 2247 & 2268 & 2385 & 2388 & 2400 & 2403 & \\
\hline Dvm2 & 2250 & 2268 & 2385 & 2388 & 2400 & 2403 & \\
\hline Exit: & 2397 & 2277 & & & & & \\
\hline Hi_10_port & 2238 & 2256 & 2259 & 2262 & 2265 & 2310 & 2406 \\
\hline$I$ & $\begin{array}{l}2283 \\
2295 \\
2331\end{array}$ & $\begin{array}{l}2286 \\
2319 \\
2331\end{array}$ & $\begin{array}{l}2286 \\
2322 \\
2334\end{array}$ & $\begin{array}{l}2289 \\
2322 \\
2334\end{array}$ & $\begin{array}{l}2292 \\
2325 \\
2337\end{array}$ & $\begin{array}{l}2292 \\
2328 \\
2337\end{array}$ & $\begin{array}{l}2295 \\
2328\end{array}$ \\
\hline Inc & 2265 & 2364 & 2364 & 2367 & 2367 & & \\
\hline Inc_i: & 2322 & 2352 & & & & & \\
\hline Inci: & 2286 & 2298 & & & & & \\
\hline Incj: & 2274 & 2373 & & & & & \\
\hline Initial: & 2409 & & & & & & \\
\hline $\mathrm{J}$ & $\begin{array}{l}2259 \\
2364\end{array}$ & 2274 & 2274 & 2277 & 2301 & 2307 & 2364 \\
\hline $\operatorname{Max} 1$ & 2328 & 2340 & 2340 & & & & \\
\hline $\operatorname{Max} 2$ & 2334 & 2346 & 2346 & & & & \\
\hline Maxs & 2313 & 2340 & 2340 & 2355 & & & \\
\hline Maxt & 2313 & 2346 & 2346 & 2358 & & & \\
\hline Minl & 2331 & 2343 & 2343 & & & & \\
\hline Min2 & 2337 & 2349 & 2349 & & & & \\
\hline Mins & 2316 & 2343 & 2343 & 2355 & & & \\
\hline Mint & 2316 & 2349 & 2349 & 2358 & & & \\
\hline
\end{tabular}




\begin{tabular}{|c|c|c|c|c|c|c|c|}
\hline Reads & $\begin{array}{l}2238 \\
2301\end{array}$ & $\begin{array}{l}2244 \\
2307\end{array}$ & $\begin{array}{l}2244 \\
2325\end{array}$ & $\begin{array}{l}2244 \\
2364\end{array}$ & 2244 & 2280 & 2289 \\
\hline Rvgl\$( & 2244 & 2280 & 2292 & & & & \\
\hline $\operatorname{Rvg} 2 \$($ & 2244 & 2280 & 2295 & & & & \\
\hline Rvgavl( & 2238 & 2301 & & & & & \\
\hline Rvgav2( & 2238 & 2307 & & & & & \\
\hline Rvgtest & 2301 & 2307 & 2376 & 2379 & & & \\
\hline Secline: & 2364 & & & & & & \\
\hline Start: & 2247 & 2394 & & & & & \\
\hline Stopj & 2253 & 2262 & 2277 & & & & \\
\hline Swtch: & 2376 & 2304 & 2310 & & & & \\
\hline Temp1( & 2244 & 2292 & 2301 & 2328 & 2328 & 2331 & 2331 \\
\hline Temp2( & 2244 & 2295 & 2307 & 2334 & 2334 & 2337 & 2337 \\
\hline Test: & 2301 & 2289 & & & & & \\
\hline Typ_flag & 2238 & 2256 & 2259 & 2262 & 2265 & 2406 & \\
\hline Varl & 2355 & 2364 & & & & & \\
\hline $\operatorname{Var} 2$ & 2358 & 2364 & & & & & \\
\hline Var: & 2355 & 2325 & & & & & \\
\hline
\end{tabular}


2421 SUB Dvms(Reads, V1\$(*), V2\$(*))

2424 OPTION BASE 1

2427 Dvm1 $=717$

2430 Dvm2 $=718$

2433 ON INT \#7,2 GOSUB Srq

2436 CONTROL MASK $7 ; 128$

2439 CARD ENABLE 7

2442 OUTPUT Dvm1, Dvm2; "@"

$2445 \quad I=1$

2448 Reads1=Reads+1

2451 Idle: !

2454 GOTO Idle

2457 Srq: $\quad$ ENTER Dvm1 BINT NOFORMAT;V1\$(I)

2460 IF I=Reads1 THEN Dvm2

2463 OUTPUT Dvm1; "Q"

2466 Dvm2: ENTER Dvm2 BINT NOFORMAT;V2\$(I)

2469 IF I=Reads1 THEN Exit

2472

2475

2478 RETURN

2481 Exit: OUTPUT Dvm1,Dvm2;"\&"

2484 SUBEND

2487 ! $* * * * * * * * * * * * * * * *$ READ POWER $* * * * * * * * * * * * * * * * * * * * * * * * * * * * *$

2490 ! FILE NAME: PREADS

! 810608-1110-.-BCY

S Dvms (

$\begin{array}{llllllll}\text { Dvm1 } & 2427 & 2442 & 2457 & 2463 & 2481 & & \\ \text { Dvm2 } & 2430 & 2442 & 2466 & 2472 & 2481 & & \\ \text { Dvm2: } & 2466 & 2460 & & & & & \\ \text { Exit: } & 2481 & 2469 & & & & & \\ \text { I } & 2445 & 2457 & 2460 & 2466 & 2469 & 2475 & 2475 \\ \text { Id1e: } & 2451 & 2454 & & & & & \\ \text { Reads } & 2421 & 2448 & & & & & \\ \text { Reads1 } & 2448 & 2460 & 2469 & & & \\ \text { Srq: } & 2457 & 2433 & & & & \\ \text { v1\$( } & 2421 & 2457 & & & & \\ \text { v2\$( } & 2421 & 2466 & & & & \end{array}$


2493 SUB Preads(Reads,Hi_lo_port, Repeat,Volts(*), Vinc(*))

2496 OPTION BASE 1

2499 DIM Decade\$ [4],V1\$(Reads+1),V2\$(Reads+1), Temp1(Reads), Temp2(Reads)

2502 ! IF Repeat=1 THEN OUTPUT 717,718 USING Initial

$2505 \quad$ Mnt=1

2508 Incr $=1$

2511 Incp\$="00"

2514 Port\$="3" ! port \#1

2517 ! IF NOT Hi_10_port THEN OUTPUT 710;"5556"

2520 Incmnt: Mnt=Mnt+1

2523

2526

IF Mnt>9 THEN Exit

Mnt $\$=V A L \$$ (Incr)

2529

OUTPUT 709; Incp\$\&Port\$\&Mnt\$

2532

IF Mnt>5 THEN Mnt789

2535

2538

CALL Dvms (Reads,V1\$(*), V2\$(*))

2541

FOR I=1 TO Reads

Temp1(I) $=\operatorname{VAL}(\operatorname{VI} \$(I+1))$ ! Mnt1

2544

2547

2550

GOSUB Ave

Temp2(I) $=\operatorname{VAL}(\operatorname{V} 2 \$(I+1)) \quad$ Mnt234

2553 GOTO Incr

2556 Mnt789: CALL Dvms(Reads,V1\$(*), V2\$(*))

2559

2562

2565

2568

2571

2574

2577

2580

2583

2586

2589

2592

2595

2598 Exit: OUTPUT $709 ; " \mathrm{Cl}$

2601 ! IF NOT Hi_1o_port THEN OUTPUT 710;"5,5254"

2604 SUBEXIT

2607 Ave: Vinc (Repeat, Mnt) $=$ SUM (Temp1)/Reads

2610 Volts (Repeat, Mnt) $=$ SUM (Temp2) $/$ Reads

2613 RETURN

2616 Initial: IMAGE "F3VR1S4T0,"

2619 SUBEND

2622 ! $* * * * * * * * * * * * * * *$ SUBPROGRAM PCALC $* * * * * * * * * * * * * * * * * * * * * * * *$

$2625 !$ FILE NAME: PCALC ! 810807-1800_-.BCY

$S$ Preads (

Ave:

$2607 \quad 2550 \quad 2571$

Decade\$

2499

Exit:

$2598 \quad 2523$ 


\begin{tabular}{|c|c|c|c|c|c|c|c|}
\hline Hi_lo_port & 2493 & & & & & & \\
\hline I & $\begin{array}{l}2538 \\
2562\end{array}$ & $\begin{array}{l}2541 \\
2562\end{array}$ & $\begin{array}{l}2541 \\
2565\end{array}$ & $\begin{array}{l}2544 \\
2565\end{array}$ & $\begin{array}{l}2544 \\
2568\end{array}$ & 2547 & 2559 \\
\hline Incmnt: & 2520 & 2577 & 2595 & & & & \\
\hline Incp\$ & 2511 & 2529 & 2586 & & & & \\
\hline Incr & 2508 & 2526 & 2574 & 2574 & 2589 & & \\
\hline Incr: & 2574 & 2553 & & & & & \\
\hline Initial: & 2616 & & & & & & \\
\hline Mnt & $\begin{array}{l}2505 \\
2580\end{array}$ & $\begin{array}{l}2520 \\
2580\end{array}$ & $\begin{array}{l}2520 \\
2607\end{array}$ & $\begin{array}{l}2523 \\
2610\end{array}$ & 2532 & 2577 & 2577 \\
\hline Mnt\$ & 2526 & 2529 & & & & & \\
\hline Mnt789: & 2556 & 2532 & & & & & \\
\hline Port\$ & 2514 & 2529 & 2583 & & & & \\
\hline Reads & $\begin{array}{l}2493 \\
2556\end{array}$ & $\begin{array}{l}2499 \\
2559\end{array}$ & $\begin{array}{l}2499 \\
2607\end{array}$ & $\begin{array}{l}2499 \\
2610\end{array}$ & 2499 & 2535 & 2538 \\
\hline Repeat & 2493 & 2607 & 2610 & & & & \\
\hline Templ( & 2499 & 2541 & 2562 & 2607 & & & \\
\hline Temp2( & 2499 & 2544 & 2565 & 2610 & & & \\
\hline V1\$( & 2499 & 2535 & 2541 & 2556 & 2565 & & \\
\hline V2\$( & 2499 & 2535 & 2544 & 2556 & 2562 & & \\
\hline Vinc( & 2493 & 2607 & & & & & \\
\hline Volts ( & 2493 & 2610 & & & & & \\
\hline
\end{tabular}


2628 SUB Pcalc(Type\$, Typ\$,Hi_lo_port, Repeats, Count, Freqs, Rvgl(*), $\operatorname{Rvg} 2(*), \operatorname{Voff1}(*), \operatorname{Voff2}(*), \operatorname{Von}(*), \operatorname{Vinc}(*), P_{-}$out $(*)$, $\operatorname{Map}(*), \operatorname{Pinc}(*), \operatorname{Vioff1}(*), \operatorname{Vioff} 2(*))$

2631 OPTION BASE 1

2634 DIM Off_sum $(1,10)$,Voff_ave $(1,10)$

2637 DIM Off_sumi $(1,10)$, Voff_avei $(1,10)$

2640 DIM On(Repeats, 10), $\operatorname{Trvg} \overline{1}(2), \operatorname{Trvg} 2(2)$

2643 Typ=POS (Typ\$, "P")

2646 MAT off_sum $=$ Voffl+Voff2

2649 MAT off sumi=Vioffl+Vioff2

2652 MAT Voff__ave=off_sum/(2)

2655 MAT Voff_avei=of $\bar{f} \_s u m i /(2)$

2658 MAT On=Von

2661 GOTO Skprt1

2664 PRINTER IS 7,1 ! FOR PRINTOUT OF

2667 MAT PRINT Voffl ! VOFFS AND VONS

2670 MAT PRINT Vioff1

2673 MAT PRINT Voff2

2676 MAT PRINT Vioff2

2679 MAT PRINT Von

2682 MAT PRINT Vinc

2685 PRINTER IS 16

2688 Skprtl: !

2691 MAT Trvgl=Rvg1*(2)

2694 MAT Trvg2=Rvg2*(2)

2697 F-Freqs

2700 Rep: Rep=Rep+1

2703 IF Rep>Repeats THEN SUBEXIT

2706 Mnt=1

2709 Mnt: Mnt $=M n t+1$

2712 IF Mnt>4 THEN Rep

2715 Inc=NOT Hi_lo_port OR (Typ=4)

2718 Sum1=On $(\operatorname{Rep}, \operatorname{Mn} t)+\operatorname{Voff}$ ave $(1, \operatorname{Mn} t)+\operatorname{Trvg} 2(1+\operatorname{Inc})$

2721

2724

2727

2730

2733

2736

2739

2742

2745

2748

2751

2754

2757

2760

2763

2766

2769

2772

2775

2778

Sumli=Vinc (Rep, Mnt)+Voff_avei $(1$, Mnt $)+\operatorname{Trvg} 1(1+\operatorname{Inc})$

Sum2 $=$ On $(\operatorname{Rep}$, Mnt +5$)+\operatorname{Voff}$ ave $(1$, Mnt +5$)+\operatorname{Trvgl}(1+\operatorname{Inc})$

Sum2 $i=V$ inc $($ Rep, Mnt +5$)+$ Voff_avei $(1$, Mnt +5$)+\operatorname{Trvg} 2(1+\operatorname{Inc})$

Dell $=$ Voff_ave $(1$, Mnt $)-$ On $(\operatorname{Re} \bar{p}$, Mnt $)$

Delli=Vof $\bar{f}_{\text {_ave }}(1$, Mnt) -Vinc (Rep, Mnt)

Del2 $=$ Voff_ave $(1, M n t+5)-$ On (Rep, Mnt +5)

$\operatorname{De} 12 i=\operatorname{Vof} \bar{f}$ avei $(1, \mathrm{Mnt}+5)-\operatorname{Vinc}(\operatorname{Rep}, \mathrm{Mnt}+5)$

IF Type $\$=$ "AIRLINE" THEN A_line

IF Mnt>2 THEN Skip

Pinc $(F, 1$, Rep, Count $)=5 *$ Sumli $*$ Delli ! for display

P_out (F, Rep, Count, 2$)=5 * \operatorname{Sum} 1 * \operatorname{De} 11 /(5 * \operatorname{Sum} 1 i *$ Delli)

Pinc $(F, 2, \operatorname{Rep}$, Count $)=5 * \operatorname{Sum} 2 i * \operatorname{De} 12 i$ !for display

GoTo Mnt

P_out (F, Rep, Count , 7) $=5 * \operatorname{Sum} 2 * \operatorname{De} 12 /(5 * \operatorname{Sum} 2 i * \operatorname{De} 12 i)$

P_out (F, Rep, Count , 1) -P_out (F, Rep, Count , 6) $=1$

Skip: Pincl $=5 *$ Suml $i *$ Delli

P_out (F, Rep, Count, Mnt) $=5 *$ Sum $1 *$ De11/Pinc1

Pinc2 $=5 *$ Sum $2 i * \operatorname{De} 12 i$

GOTO Mnt

P_out (F, Rep, Count , Mnt +5$)=5 *$ Sum $2 *$ De $12 /$ Pinc2

2781 A_line: IF Mnt $>2$ THEN Skp

2784

$\operatorname{Pinc}(\operatorname{Map}(F), 1, \operatorname{Rep}$, Count $)=5 * \operatorname{Sum} 1 i * \operatorname{Dell} i$ 
$2787 \operatorname{Pinc}(\operatorname{Map}(F), 2, \operatorname{Rep}$, Count $)=5 * \operatorname{Sum} 2 i * \operatorname{De} 12 i$

$2790 \quad$ P_out $(\operatorname{Map}(F), \operatorname{Rep}$, Count , 1) $=P$ out $(\operatorname{Map}(F), \operatorname{Rep}$, Count , 6) $=1$

2793 P_out (Map (F), Rep, Count, 2) $=5 * \operatorname{Sum} 1 * \operatorname{De} 11 /(5 * \operatorname{Sum} 1 \mathrm{D} * \operatorname{De} 11 i)$

2796 P_out (Map (F), Rep, Count , 7) $=5 * \operatorname{Sum} 2 * \operatorname{De} 12 /(5 * \operatorname{Sum} 2 i * \operatorname{De} 12 i)$

2799 GoTo Mnt

2802 Skp: Pincl=5*Sumli*Delli

$2805 \quad$ Pinc $2=5 *$ Sum $2 i *$ De12i

2808 P out (Map (F), Rep, Count, Mnt $)=5 *$ Suml $1 * \operatorname{De} 11 /$ Pincl

2811 P_out (Map (F), Rep, Count, Mnt +5) $=5 *$ Sum $2 * \operatorname{De} 12 /$ Pinc 2

2814 GOTO Mnt

2817 SUBEND

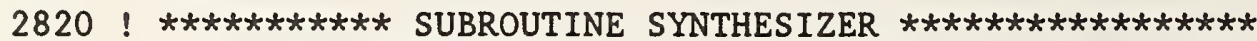

2823 ! FILE NAME: SYNTH ! 811208-1700-.-DHR \& BCY

S Pcalc(

\begin{tabular}{|c|c|c|c|c|c|c|c|}
\hline A_1ine: & 2781 & 2742 & & & & & \\
\hline \multirow[t]{3}{*}{ Count } & 2628 & 2748 & 2751 & 2754 & 2757 & 2760 & 2760 \\
\hline & 2769 & 2775 & 2784 & 2787 & 2790 & 2790 & 2793 \\
\hline & 2796 & 2808 & 2811 & & & & \\
\hline De11 & 2730 & 2751 & 2769 & 2793 & 2808 & & \\
\hline De11i & 2733 & 2748 & 2751 & 2766 & 2784 & 2793 & 2802 \\
\hline De12 & 2736 & 2757 & 2775 & 2796 & 2811 & & \\
\hline $\operatorname{De} 12 i$ & 2739 & 2754 & 2757 & 2772 & 2787 & 2796 & 2.805 \\
\hline \multirow[t]{3}{*}{$F$} & 2697 & 2748 & 2751 & 2754 & 2757 & 2760 & 2760 \\
\hline & 2769 & 2775 & 2784 & 2787 & 2790 & 2790 & 2793 \\
\hline & 2796 & 2808 & 2811 & & & & \\
\hline Freqs & 2628 & 2697 & & & & & \\
\hline Hi_1o_port & 2628 & 2715 & & & & & \\
\hline Inc & 2715 & 2718 & 2721 & 2724 & 2727 & & \\
\hline \multirow[t]{2}{*}{$\operatorname{Map}($} & 2628 & 2784 & 2787 & 2790 & 2790 & 2793 & 2796 \\
\hline & 2000 & 2011 & & & & & \\
\hline \multirow[t]{4}{*}{ Mnt } & 2706 & 2709 & 2709 & 2712 & 2718 & 2718 & 2721 \\
\hline & 2721 & 2724 & 2724 & 2727 & 2727 & 2730 & 2730 \\
\hline & 2733 & 2733 & 2736 & 2736 & 2739 & 2739 & 2745 \\
\hline & 2769 & 2775 & 2781 & 2808 & 2811 & & \\
\hline Mnt: & 2709 & 2763 & 2778 & 2799 & 2814 & & \\
\hline Off_sum( & 2634 & 2646 & 2652 & & & & \\
\hline off_sumi( & 2637 & 2649 & 2655 & & & & \\
\hline
\end{tabular}




\begin{tabular}{|c|c|c|c|c|c|c|c|}
\hline On & 2640 & 2658 & 2718 & 2724 & 2730 & 2736 & \\
\hline P_out ( & $\begin{array}{l}2628 \\
2790\end{array}$ & $\begin{array}{l}2751 \\
2790\end{array}$ & $\begin{array}{l}2757 \\
2793\end{array}$ & $\begin{array}{l}2760 \\
2796\end{array}$ & $\begin{array}{l}2760 \\
2808\end{array}$ & $\begin{array}{l}2769 \\
2811\end{array}$ & 2775 \\
\hline Pincl & 2628 & 2748 & 2754 & 2784 & 2787 & & \\
\hline Pincl & 2766 & 2769 & 2802 & 2808 & & & \\
\hline Pinc2 & 2772 & 2775 & 2805 & 2811 & & & \\
\hline Rep & $\begin{array}{l}2700 \\
2730 \\
2757 \\
2790\end{array}$ & $\begin{array}{l}2700 \\
2733 \\
2760 \\
2790\end{array}$ & $\begin{array}{l}2703 \\
2736 \\
2760 \\
2793\end{array}$ & $\begin{array}{l}2718 \\
2739 \\
2769 \\
2796\end{array}$ & $\begin{array}{l}2721 \\
2748 \\
2775 \\
2808\end{array}$ & $\begin{array}{l}2724 \\
2751 \\
2784 \\
2811\end{array}$ & $\begin{array}{l}2727 \\
2754 \\
2787\end{array}$ \\
\hline Rep: & 2700 & 2712 & & & & & \\
\hline Repeats & 2628 & 2640 & 2703 & & & & \\
\hline Rvg1( & 2628 & 2691 & & & & & \\
\hline Rvg2( & 2628 & 2694 & & & & & \\
\hline Skip: & 2766 & 2745 & & & & & \\
\hline Skp : & 2802 & 2781 & & & & & \\
\hline Skprt1: & 2688 & 2661 & & & & & \\
\hline Sum1 & 2718 & 2751 & 2769 & 2793 & 2808 & & \\
\hline Sum1i & 2721 & 2748 & 2751 & 2766 & 2784 & 2793 & 2802 \\
\hline Sum2 & 2724 & 2757 & 2775 & 2796 & 2811 & & \\
\hline Sum2i & 2727 & 2754 & 2757 & 2772 & 2787 & 2796 & 2805 \\
\hline Trvg1( & 2640 & 2691 & 2721 & 2724 & & & \\
\hline Trvg2( & 2640 & 2694 & 2718 & 2727 & & & \\
\hline Typ & 2643 & 2715 & & & & & \\
\hline Typ\$ & 2628 & 2643 & & & & & \\
\hline Types & 2628 & 2742 & & & & & \\
\hline Vince & 2628 & 2682 & 2721 & 2727 & 2733 & 2739 & \\
\hline Vioffl( & 2628 & 2649 & 2670 & & & & \\
\hline Vioff2( & 2628 & 2649 & 2676 & & & & \\
\hline Voffl( & 2628 & 2646 & 2667 & & & & \\
\hline
\end{tabular}




$\begin{array}{lllllll}\text { Voff2( } & 2628 & 2646 & 2673 & & & \\ \text { Voff_ave( } & 2634 & 2652 & 2718 & 2724 & 2730 & 2736 \\ \text { Voff_avei( } & 2637 & 2655 & 2721 & 2727 & 2733 & 2739 \\ \text { Von( } & 2628 & 2658 & 2679 & & & \end{array}$


2826 SUB Synth(Mode, Freq, Last_freq, Level)

2827 ! INSERTED GO TO AND CALL STATEMENT AT 2830 AND 2928

RESP. 8507241443 GJC

2829 Addr $=728$

2832 IF Leve1>6 THEN DISP "TOO MUCH SYNTHESIZER LEVEL CONTROL - - PROGRAM HALT"

2835 IF Leve1>6 THEN STOP

2838 Lv1=Leve1

2841 Freq mhz-Freq*1E6

2844 IF (Freq $>-2)$ AND (Last_freq $<2)$ OR (Freq $<2$ ) AND (Last_freq $>$

-2) THEN Attn flag=1

2847 IF Attn flag THEN Attn $=6$

2850 IF Attn flag THEN Lo lvl

2853 Attn=INT (ABS $(\operatorname{Lvl} / 10))+2$

2856 Lo lv1: Lo $1 \mathrm{vl}=350$

2859 OUTPिUT Addr USING Lvlout;Attn, Lo_1v1

2862

OUTPUT Addr USING Mode;Mode

OUTPUT Addr USING Freq; Freq mhz

IF Attn_flag THEN Attn-INT(ABS(Lv1/10)) +2

2868

Lvlout $=\bar{A} B S(\operatorname{Lv} 1 * 10-240)-($ Attn -1$) * 100-(\operatorname{Lvl}>0)$

2871

2874

2877

2880

OUTPUT Addr USING Lvlout;Attn, Lvlout

Status $\$=\operatorname{VAL} \$(4)$

2883

ENTER Addr; Status\$

2886

Temp-VAL(Status\$)

IF NOT Temp THEN SUBEXIT

$\mathrm{Cnt}=\mathrm{Cnt}+1$

IF Cnt $<101$ THEN Status

IF Temp=1 THEN PRINT "UNLOCKED"; CHR $\$(7)$

IF Temp=2 THEN PRINT "UNLEVELED"; CHR\$(7)

IF Temp=3 THEN PRINT "UNLOCKED \& UNLEVELED";CHR\$(7)

Countr=Countr+1

IF Countr $<51$ THEN Status

DISP "DO YOU WANT TO CONTINUE ? - ( $(\mathrm{Y} / \mathrm{N}) "$; $\operatorname{CHR} \$(7)$;

Cnt=Countr $=0$

IF FNYorn THEN Status

2916

2919 STOP

2922 Mode: IMAGE "O",D

2925 Freq: IMAGE "H",8Z

2928 Lvlout: IMAGE "N",D, $3 Z$

2933 SUBEND

2934 ! ************** SUBPROGRAM FILTER $* * * * * * * * * * * * * * * * * * * * * * * * * * * * * * 4$

2937 ! FILE NAME: FILTER ! 810522-1630_-BCY

S Synth(

$\begin{array}{lllllll}\text { Addr } & 2829 & 2859 & 2862 & 2865 & 2874 & 2880 \\ \text { Attn } & 2847 & 2853 & 2859 & 2868 & 2871 & 2874 \\ \text { Attn_flag } & 2844 & 2847 & 2850 & 2868 & & \\ \text { Cnt } & 2889 & 2889 & 2892 & 2913 & & \\ \text { Countr } & 2904 & 2904 & 2907 & 2913 & \end{array}$




\begin{tabular}{|c|c|c|c|c|c|}
\hline Freq & 2826 & 2841 & 2844 & 2844 & \\
\hline Freq: & 2925 & & & & \\
\hline Freq_mhz & 2841 & 2865 & & & \\
\hline Last_freq & 2826 & 2844 & 2844 & & \\
\hline Level & 2826 & 2832 & 2835 & 2838 & \\
\hline Lo_1v1 & 2856 & 2859 & & & \\
\hline Lo_1v1: & 2856 & 2850 & & & \\
\hline Lv1 & 2838 & 2853 & 2868 & 2871 & 2871 \\
\hline Lvlout & 2871 & 2874 & & & \\
\hline Lvlout: & 2928 & & & & \\
\hline Mode & 2826 & 2862 & & & \\
\hline Mode: & 2922 & & & & \\
\hline Status\$ & 2877 & 2880 & 2883 & & \\
\hline Status: & 2880 & 2892 & 2907 & 2916 & \\
\hline Temp & 2883 & 2886 & 2895 & 2898 & 2901 \\
\hline
\end{tabular}


2940 SUB Filter(Path)

2943 DIM Table\$(12,2) [6], Data\$ [6], Pos\$[2]

2946 Scanner $=710$

2949 Table $(1,1)=$ "606162"

2952 Table $(1,2)=$ Nul1\$

2955 Table $(2,1)=" 6061 "$

2958 Table $(2,2)=" 62 "$

2961 Table $\$(3,1)=" 6063 "$

2964 Table $(3,2)=" 61 "$

2967 Table $(4,1)=" 60 "$

2970 Table $\$(4,2)=" 6163 "$

2973 Table $(5,1)=" 64$ "

2976 Table $(5,2)=" 60 "$

2979 Table $\$(6,1)=\operatorname{Nul} 1 \$$

2982 Table $\$(6,2)=" 6064$ "

2985 Table $\$(7,1)=$ "656667"

2988 Table $(7,2)=$ Nul1 $\$$

2991 Table $(8,1)=" 6566 "$

2994 Table $(8,2)=" 67 "$

2997 Table $(9,1)=" 6568 "$

3000 Table $(9,2)=" 66 "$

3003 Table $\$(10,1)=" 65 "$

3006 Table $(10,2)=" 6668 "$

3009 Table $(11,1)={ }^{n} 69$ "

3012 Table $(11,2)=" 65 "$

3015 Table $(12,1)=$ Nu11 $\$$

3018 Table $(12,2)=" 6569 "$

3021 Pos $\$=\operatorname{VAL} \$(40+($ Path $>6))$

3024 OUTPUT Scanner USING "4(K/)";"70";Pos\$;"4";"7"

3027 Pos: Pos $=$ Pos +1

3030 IF Pos $>2$ THEN SUBEXIT

3033 Data\$=Table\$(Path, Pos)

3036 IF Data\$=Nuli\$ THEN Pos

3039 Pos $\$=\operatorname{VAL} \$(40+($ Pos $=2))$

3042 OUTPUT Scanner USING "4(K/) ";Data\$;Pos\$;"4";"6"

3045 GOTO POS

3048 SUBEND

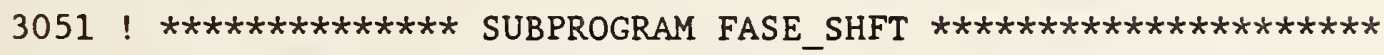
3054 ! FILE NAME: FASE

! 810522-1810--BCY

S Filter(

$\begin{array}{llllll}\text { Data\$ } & 2943 & 3033 & 3036 & 3042 & \\ \text { Nul1\$ } & 2952 & 2979 & 2988 & 3015 & 3036 \\ \text { Path } & 2940 & 3021 & 3033 & & \\ \text { Pos } & 3027 & 3027 & 3030 & 3033 & 3039 \\ \text { Pos\$ } & 2943 & 3021 & 3024 & 3039 & 3042 \\ \text { Pos: } & 3027 & 3036 & 3045 & & \end{array}$


Scanner

Table\$(
$2946 \quad 3024 \quad 3042$

$\begin{array}{lllllll}2943 & 2949 & 2952 & 2955 & 2958 & 2961 & 2964\end{array}$

$\begin{array}{lllllll}2967 & 2970 & 2973 & 2976 & 2979 & 2982 & 2985\end{array}$

$\begin{array}{lllllll}2988 & 2991 & 2994 & 2997 & 3000 & 3003 & 3006\end{array}$

$\begin{array}{lllll}3009 & 3012 & 3015 & 3018 & 3033\end{array}$ 
3057 SUB Fase_shft(I,Hi_lo_port)

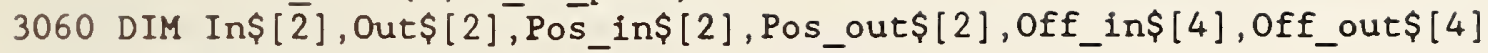

3063 Temp-4*Hi_10_port

3066 In\$-VAL\$ ( $\overline{4} 3+\bar{T}$ emp $)$

3069 Out\$-VAL\$ (44+Temp)

3072 Pos_in\$=VAL\$ $(40+((I=1)$ OR $(I=2)))$

3075 Pos_out $\$=\operatorname{VAL} \$(40+((I=1)$ OR $(I=4)))$

3078 Off_in\$=" 4, , \&In\$

3081 off_out\$="4, "\&Out\$

3084 OUTPUT 710 USING " $8(\mathrm{~K} /)^{n}$;In\$;Pos_in\$;Off_in\$; "4";Out\$; Pos_out\$; Off_out\$; 4 "

3087 SUBEND

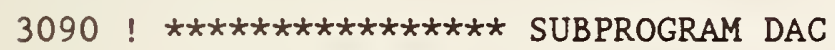

3093 ! FILE NAME: DAC

! 810203-1830---BCY

S Fase_shft(

$\begin{array}{llllll}\text { Hi_lo_port } & 3057 & 3063 & & & \\ \text { I } & 3057 & 3072 & 3072 & 3075 & 3075 \\ \text { In\$ } & 3060 & 3066 & 3078 & 3084 & \\ \text { off_in\$ } & 3060 & 3078 & 3084 & & \\ \text { off_out\$ } & 3060 & 3081 & 3084 & \\ \text { Out\$ } & 3060 & 3069 & 3081 & 3084 \\ \text { Pos_in\$ } & 3060 & 3072 & 3084 & \\ \text { Pos_out\$ } & 3060 & 3075 & 3084 & \\ \text { Temp } & 3063 & 3066 & 3069 & \end{array}$


3096 SUB Dac(Volts,Dac_err)

3099 Dac $=706$

3102 Dac_err $=0$

3105 IF (V̄olts $<0)$ OR NOT (Volts $<1$ ) THEN Dac_err=1

3108 IF Dac_err THEN SUBEXIT

3111 Data\$=VAL\$ $(1000+\operatorname{INT}(\operatorname{Volts} / .001+.5))$

3114 OUTPUT Dac USING "\#,K";Data\$

3117 SENDBUS Dac;191

3120 SUBEND

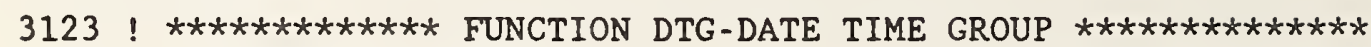

3126 ! FILE NAME: FNDTG\$ ! 80513-17300 - -BCY

S Dac(

$\begin{array}{lllll}\text { Dac } & 3099 & 3114 & 3117 & \\ \text { Dac_err } & 3096 & 3102 & 3105 & 3108 \\ \text { Data\$ } & 3111 & 3114 & & \\ \text { Volts } & 3096 & 3105 & 3105 & 3111\end{array}$


3129 DEF FNDEg\$

3130 ! !!!!!CHANGED DATE FROM 84 TO 85 850724:GJC

3132 OUTPUT 9; "Read"

3135 ENTER 9;AS

3138 Dtg\$-"85"\&A\$[1,2]\&A\$[4,5]\&" . "\&A\$ [ 7, 8]\&A\$[10,11]\&A\$[13, 14]

3141 RETURN Dtg\$

3144 FNEND

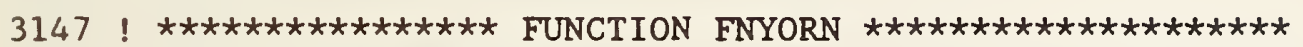

3150 ! FILE NAME: FNYORN

! 810101-1600---BCY

FNDtg\$

$\begin{array}{lllllll}\text { A } & 3135 & 3138 & 3138 & 3138 & 3138 & 3138 \\ \text { Dtg\$ } & 3138 & 3141 & & & & \end{array}$


3153 DEF FNYorn

3156 Key: Key=READBIN (0)

3159

3162

N-Answer=NOT $(($ Key=78) OR $($ Key=110))

3165

$\mathrm{Y}=$ Answer $=($ Key=89) OR $($ Key $=121)$

3168

IF NOT $Y$ AND $N$ THEN Key

3171 RETURN Answer

3174 FNEND

$3177 * * * * * * * * * * * * * * * * * * *$ FUNCTION FNNUMI ********************

3180 ! FILE NAME: FNNUMI

! 810101-1600--BCY

FNYorn

$\begin{array}{llllll}\text { Answer } & 3159 & 3162 & 3171 & & \\ \text { Key } & 3156 & 3159 & 3159 & 3162 & 3162 \\ \text { Key: } & 3156 & 3165 & & & \\ \text { N } & 3159 & 3165 & & & \\ \text { Y } & 3162 & 3165 & & & \end{array}$


3183 DEF FNNumi(Linput\$)

3186 Length-LEN (TRIMS (Linput\$))

3189 IF NOT Length THEN RETURN 0

3192 Inci: $\mathrm{I}=\mathrm{I}+1$

3195 IF I>Length THEN RETURN 1

3198 Num=NUM (Linput\$ [I])

3201 IF (Num< $<47$ ) OR (Num $>=58$ ) THEN RETURN 0

3204 GOTO Inci

3207 FNEND

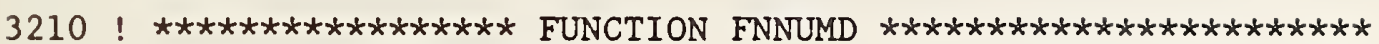
3213 ! FILE NAME: FNNUMD

! 820302-1700---BCY

FNNumi(

$\begin{array}{lllll}\text { I } & 3192 & 3192 & 3195 & 3198 \\ \text { Inc1: } & 3192 & 3204 & & \\ \text { Length } & 3186 & 3189 & 3195 & \\ \text { Linput\$ } & 3183 & 3186 & 3198 & \\ \text { Num } & 3198 & 3201 & 3201\end{array}$


3216 DEF FNNumd(Linput\$)

3219 Dec $=0$

3222 Len=LEN (TRIMS (Linput\$))

3225 IF NOT Len $=0$ THEN RETURN 0

! length

3228 Inci: $I=I+1$

3231 IF I>Len THEN RETURN 1

3234 Num=NUM (Linput\$ [I])

3237 IF Num=46 THEN Test

! non-ASCII

3240

3243

3246 Test: IF Num=46 THEN Dec=Dec+1 GOTO Inci

! passes ok

! decimal value

! test decimal point

3249

3252

IF (Dec $>1$ ) OR (LEN(Linput $\$$ ) $=1$ ) THEN RETURN 0 ! none or too many or

GOTO Inci SUBEND

3258 ! *************** SUBPROGRAM ORDER $* * * * * * * * * * * * * * * * * * * * * * * * * * * *$

3261 I FILE NAME: ORDER

! 820107-1530---BCY

FNNumd(

$\begin{array}{llllll}\text { Dec } & 3219 & 3246 & 3246 & 3249 & \\ \text { I } & 3228 & 3228 & 3231 & 3234 & \\ \text { Inci: } & 3228 & 3243 & 3252 & & \\ \text { Len } & 3222 & 3225 & 3231 & & \\ \text { Linput\$ } & 3216 & 3222 & 3234 & 3249 & \\ \text { Num } & 3234 & 3237 & 3240 & 3240 & 3246 \\ \text { Test: } & 3246 & 3237 & & & \end{array}$


3264 SUB Order(F(*), Nfreqs)

3267 OPTION BASE 1

3270 IF Nfreqs=1 THEN SUBEXIT

3273 Stop_i-INT(Nfreqs/2)

3276 Inc i : I-J-I+1

3279 - IF I>Stop_i THEN SUBEXIT

$3282 \quad \operatorname{Min}=\operatorname{Max}=\bar{F}(\mathrm{I})$

$3285 \quad \mathrm{~L}=\mathrm{K}=0$

3288

3291

3294

3297

3300

3303

3306

3309

3312

3315

3318

3321

3324

3327

3330

3333

3336

3339

3342

Inc $j: \mathrm{J}=\overline{\mathrm{J}}+1$

$$
\text { Stop_j-Nfreqs - I+1 }
$$

IF J>Stop_j THEN Swtch

Compare $=F(\mathrm{~J})$

IF Compare>Min THEN Max

$\mathrm{K}=\mathrm{J}$

Min-Compare

Max: IF Compare $<$ Max THEN Inc_j

$\mathrm{L}=\mathrm{J}$

Max=Compare

GoTO Inc_j

Swtch: IF $F(I)=$ Min THEN Skip

$F(K)=F(I)$

$F(I)=M$ in

Skip: IF $F$ (Stop_j)=Max THEN Inc_i IF NOT L THEN $L=K$ $F(L)=F($ Stop_j)

GOTO Inc i

$F($ Stop_j) $=$ Max

3345 SUBEND

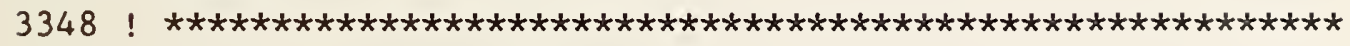

S Order (

$\begin{array}{llllllll}\text { Compare } & 3297 & 3300 & 3306 & 3309 & 3315 & & \\ \text { F( } & 3264 & 3282 & 3297 & 3321 & 3324 & 3324 & 3327 \\ & 3330 & 3336 & 3336 & 3339 & & & \\ \text { I } & 3276 & 3276 & 3279 & 3282 & 3288 & 3321 & 3324 \\ & 3327 & & & & & & \\ \text { Inc_i: } & 3276 & 3330 & 3342 & & & & \\ \text { Inc_j: } & 3291 & 3309 & 3318 & & & & \\ \text { J } & 3276 & 3291 & 3291 & 3294 & 3297 & 3303 & \\ \text { K } & 3285 & 3303 & 3324 & 3333 & & & \\ \text { L } & 3285 & 3312 & 3333 & 3333 & 3336 & & \\ \text { Max } & 3282 & 3309 & 3315 & 3330 & 3339 & & \\ \text { Max: } & 3309 & 3300 & & & & & \end{array}$




$\begin{array}{llllll}\text { Min } & 3282 & 3300 & 3306 & 3321 & 3327 \\ \text { Nfreqs } & 3264 & 3270 & 3273 & 3288 & \\ \text { Skip: } & 3330 & 3321 & & & \\ \text { Stop_1 } & 3273 & 3279 & & & \\ \text { Stop_j } & 3288 & 3294 & 3330 & 3336 & 3339 \\ \text { Swtch: } & 3321 & 3294 & & & \end{array}$


3351 SUB Avg(Ave(*), $\operatorname{Var}(*), X(*), N)$

! 811108-0900-..-BCY

3354 OPTION BASE 1

3357 MAT Var-Var* $(\mathrm{N}-2)$

3360 MAT $X=X$-Ave

3363 MAT $X=X /(N)$

3366 MAT Ave-X+Ave

3369 MAT $X-X . X$

3372 MAT $\mathrm{X}=\mathrm{X} *((\mathrm{~N}-1) * \mathrm{~N})$

3375 MAT Var-Var $+\mathrm{X}$

3378 MAT Var=Var $/(\mathrm{N}-1)$

3381 SUBEND

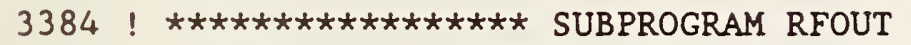

3387 ! FILE NAME: RFOUT

! 820130-1300-..BCY

$\operatorname{S~} \operatorname{Avg}($

Ave ( $\quad 3351 \quad 3360 \quad 3366 \quad 3366$

$\begin{array}{lllllll}\mathrm{N} & 3351 & 3357 & 3363 & 3372 & 3372 & 3378\end{array}$

$\begin{array}{llllllll}\operatorname{Var}( & 3351 & 3357 & 3357 & 3375 & 3375 & 3378 & 3378\end{array}$

$\begin{array}{llllllll}X( & 3351 & 3360 & 3360 & 3363 & 3363 & 3366 & 3369\end{array}$ 
3390 SUB Rfout(Kind\$, Path(*), Freq(*), Freqs, Start, Hilo)

3393 OPTION BASE 1

3396 Mode $=0$

3399 IF Kind\$="NEXT" THEN Next

3402 IF Kind\$="FIRST" THEN First

3405 N_freq=L_freq=Freq (1)

3408 PathePath(1)

3411 GOTO Call

3414 First:N_freq=Freq(Start)

3417

3420

3423 GOTO Call

L_freq=Freq (Freqs)

PathePath(Start)

3426 Next:N_freq-Freq (Freqs+1)

3429 L_freq=Freq (Freqs)

3432 Path=Path (Freqs+1)

3435 Cal1: CALL Filter(Path)

3438 CALL Pset(N_freq,Hilo,Dac_volt, Level)

3441 CALL Dac (Dac_volt,Err)

3444 CALL Synth(Möde,N_freq,L_freq, Level)

3447 SUBEND

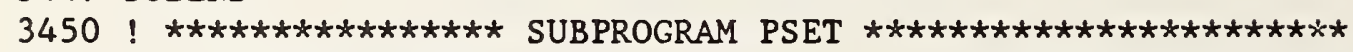

3453 ! FILE NAME: PSET ! 820000-0000---BCY

$3454 ! \star \star \star \star \star \star \star \star \star *$ RENAMED SUB PSET TO PSETDEL $850724: 1446$ :GJC

S Rfout(

\begin{tabular}{|c|c|c|c|c|c|c|}
\hline Call: & 3435 & 3411 & 3423 & & & \\
\hline Dac_volt & 3438 & 3441 & & & & \\
\hline Err & 3441 & & & & & \\
\hline First: & 3414 & 3402 & & & & \\
\hline Freq( & 3390 & 3405 & 3414 & 3417 & 3426 & 3429 \\
\hline Freqs & 3390 & 3417 & 3426 & 3429 & 3432 & \\
\hline Hilo & 3390 & 3438 & & & & \\
\hline KindS & 3390 & 3399 & 3402 & & & \\
\hline L_freq & 3405 & 3417 & 3429 & 3444 & & \\
\hline Leve1 & 3438 & 3444 & & & & \\
\hline Mode & 3396 & 3444 & & & & \\
\hline $\mathrm{N}_{\text {_freq }}$ & 3405 & 3414 & 3426 & 3438 & 3444 & \\
\hline Next: & 3426 & 3399 & & & & \\
\hline Path & 3408 & 3420 & 3432 & 3435 & & \\
\hline Path( & 3390 & 3408 & 3420 & 3432 & & \\
\hline Start & 3390 & 3414 & 3420 & & & \\
\hline
\end{tabular}


3456 SUB Pset(Frq,Hi_10,Dac_volt, Level)
IF (Frq $<=1$ ) AND NOT Hi_10 THEN Range00
IF (Frq $<=2$ ) AND NOT Hi_10 THEN Range0
IF Frq $<-4$ THEN Flag $=1$
3468
3471
3472
3477
3480
3483
3486
IF $($ Frq $>4$ ) AND (Frq $<=7.5$ ) THEN Flag=2
IF (Frq $>7.5$ ) AND (Frq $<=7.8$ ) THEN Flag=3
IF (Frq $>7.8$ ) AND (Frq $<8$ ) THEN Flag=4
IF $($ Frq $>-8)$ AND (Frq $<=10.5)$ THEN Flag $=5$
IF (Frq $>10.5)$ AND (Frq< $=10.9$ ) THEN Flag=6
IF (Frq $>10.9$ ) AND (Frq<12) THEN Flag=7
IF $($ Frq $>12)$ AND $($ Frq<14.0) THEN Flag $=8$
IF $($ Frq $>-14.0)$ AND $($ Frq $<=15.9)$ THEN Flag=9
IF (Frq $>15.9$ ) AND (Frq $<=16.9$ ) THEN Flag=10
IF Frq $>16.9$ THEN Flag=11
ON Flag GOTO Rangel, Range2, Range3, Range4, Range5,

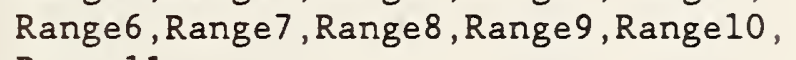

\section{Rangel1}

3498 Range00: Dac_volt $=.60$

3501

3504 SUBEXIT

3507 Range0: Dac_volt $=.55$

3510

3513

3516 Rangel: Dac_volt $=.63$

3525 Range2: Dac_volt $=.59$

3534 Range 3: Dac volt $=.59$

3543 Range4: Dac_volt $t=.59$

3546

3552 Range 5: Dac_volt $=.59$

3555

3558

3561 Range6: Dac_volt=.59

3564

3567

3570 Range 7: Dac_volt $=.63$

3573 Level $=-15$

3576 SUBEXIT

3579 Range8: Dac_volt $=.63$

3582

3585

3588 Range 9: Dac_volt $=.63$

3591

3594 SUBEXIT

3597 Range10: Dac_volt $=.63$

3601 Range11: Dac_volt $=.63$

3602

Level $=6$ 
3603 SUBEND

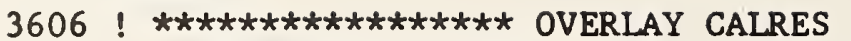

3609 ! FILE NAME: CALRES

! 810923-1330-.-BCY

S Pset(

Dac_volt

$\begin{array}{lllllll}3456 & 3498 & 3507 & 3516 & 3525 & 3534 & 3543\end{array}$

$\begin{array}{lllllll}3552 & 3561 & 3570 & 3579 & 3588 & 3597 & 3601\end{array}$

Flag

$\begin{array}{lllllll}3465 & 3468 & 3471 & 3472 & 3477 & 3480 & 3483\end{array}$

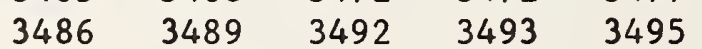

Frq

$\begin{array}{lllllll}3456 & 3459 & 3462 & 3465 & 3468 & 3468 & 3471 \\ 3471 & 3472 & 3472 & 3477 & 3477 & 3480 & 3480 \\ 3483 & 3483 & 3486 & 3486 & 3489 & 3489 & 3492 \\ 3492 & 3493 & & & & & \end{array}$

HI_10

$3456 \quad 3459 \quad 3462$

Leve1

$\begin{array}{lllllll}3456 & 3501 & 3510 & 3519 & 3528 & 3537 & 3546\end{array}$

$\begin{array}{lllllll}3555 & 3564 & 3573 & 3582 & 3591 & 3598 & 3602\end{array}$

Range 00: $\quad 3498 \quad 3459$

Range 0: $\quad 3507 \quad 3462$

Range 10: $\quad 3597 \quad 3495$

Range11: $\quad 3601 \quad 3495$

Rangel: $\quad 3516 \quad 3495$

Range2: $\quad 3525 \quad 3495$

Range 3: $\quad 3534 \quad 3495$

Range 4: $\quad 3543 \quad 3495$

Range 5: $\quad 3552 \quad 3495$

Range6: $\quad 3561 \quad 3495$

Range 7: $\quad 3570 \quad 3495$

Range 8: $\quad 3579 \quad 3495$

Range 9: $\quad 3588 \quad 3495$ 
3612 SuB Calres(Max_pts,Nfreqs, Repeats,Dim, Typ\$,Sp,Std,Hi_1o_port,

3615 OPTION BASE 1

$\operatorname{Pr} t \overline{\$}, \mathrm{Cal}, \mathrm{Gl}, \mathrm{Ca} \$$ )

3618 DIM Date $\{[6]$, Time $[6], D(16,8)$, Id_cont $\$[80]$

3621 DIM Freqs(Nfreqs), Power(Nfreqs, Repeats, Dim, 10), File\$[11], Dsk_nam\$[11]

3624 DIM Result(Nfreqs, 60), Omitf(Nfreqs), Aver(87), Var(87), Rd(Nfreqs, 2,12)

3625 Ca\$=VAL\$(Cal)

3626 Freq $=0$

3627 DISP " "

3628 From_file $=$ "PWR" \&"D"\&Ca\$

3629 F_oh_file $\$=$ "PWR " \&"H" \&Ca\$

3630 PRINTER IS 16

3636 IF Ca\$ $=$ " 3" THEN From_file $\$=" P W R D 2 "$

3637 IF Ca\$=" 3" THEN F_oh_file $\$=" P W R H 2 "$

3638 GOTO 3641

3639 ! From_file $\$=" P W R " \& " D " \& C a \$$

3640 ! F_oh_file $\$=" P W R " \& " H " \& C a \$$

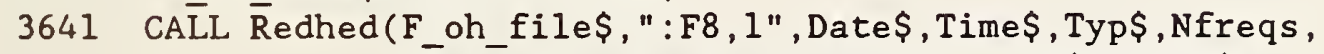
Freqs $(*)$, Repeats, Sp,Std,Id_cont\$, Cnnct\$)

3642 FREAD From_file\$\&":F8, 1",Power(*)

3643 IF GIO1 THEN GOTO 3659

3644 Fr_file $\$="$ CRST" $\& \operatorname{VAL} \$(\operatorname{VAL}(\mathrm{Ca} \$)-2)$

3645 PRINT Fr file\$

3646 CALl Redhed(Fr_file\$, ":F8, I", Date\$, Time\$, Typ\$,Nfreqs,

Frēqs (*), Repeats, Sp, Std,Id_cont\$,Cnnct $\$$ )

3647 CALL Redwrt(Fr_file\$,":F8,1"," ",Result(*), Nfreqs)

3648 FOR Fr=1 TO Nfreqs

3649 FOR $\mathrm{Sp}=1$ TO 2

3650 FOR $\mathrm{P}=1$ TO 5

3652 ON SP GOTO 3653,3655

$3653 \operatorname{Rd}(\mathrm{Fr}, \mathrm{Sp}, \mathrm{P})=\operatorname{Result}(\mathrm{Fr}, \mathrm{P})$

3654 GOTO 3656

$3655 \operatorname{Rd}(\mathrm{Fr}, \mathrm{SP}, \mathrm{P})=\operatorname{Result}(\mathrm{Fr}, \mathrm{P}+12)$

3656 NEXT $P$

3657 NEXT SP

3658 NEXT Fr

3659 Dsk_nam\$=" SCRATCH"

3660 To_f file $\$=" C R S T " \& C a \$$

3661 ON ERROR GOSUB Xerr $^{-}$

3662 ASSIGN \#9 TO "T_RSLT: F8, 1", Check

3663 ASSIGN * TO \#9

3666 IF Check THEN CALL T_disc("T_RSLT:F8, 1" , 4, 696)

3669 OFF ERROR

3672 DISP "CALCULATING SYSTEM PARAMETERS"

3675 PRINT LIN(1);"SCRATCH DATA FILE NAME: ";From_file\$

3678 PRINT LIN(1);"POWER DATA TAKEN: ";Date\$\&" "\&Time\$

3681 PRINT LIN(1);"FILE IDENTIFIER: "; Id_cont\$;LIN(1)

3684 Incfrq: Freq=Freq+1

3687

3690

IF Freq $>$ Nfreqs THEN Done

Repeat $=0$

3693 Incr: Repeat=Repeat+1 
3702 Inci: $I=I+1$

3705 IF I>16 THEN Subr

$3708 \quad$ Mnt $=0$

3711 Incm: Mnt=Mnt+1

3714 IF Mnt>8 THEN Inci

$3717 \quad \mathrm{D}(\mathrm{I}, \mathrm{Mnt})=$ Power (Freq, Repeat, I, Mnt)

3720 GOTO Incm

3723 Subr: Fghz=Freqs(Freq)

3727 CALL Coeff(Freq, Fghz, Repeat, Repeats, Date\$, Time\$, Typ, Sp, From_file\$, Dsk_nam\$, Hi_lo_port, Prt\$, Cnnct\$,

3729 GOTO Incr Errcñt, Error_fílag, D(*), Result(*), Aver(*), Var (*), Gl, $\operatorname{Rd}(*)$ )

3732

3735

Done: DISP " "

3738 IF NOT Error_flag THEN 3740

3740 IF $G 1=1$ THEN To_file $\$=" A D P " \& C a \$$

3741 IF $G 1=1$ THEN GOTO Record

3742 IF Errcnt=Nfreqs THEN Exit_flag=1

3744 IF Exit_flag THEN PRINT $\operatorname{LIN}(2)$; CHR\$(7); "NO RESULTS SAVED ... PAUSE IN EFFECT"

3747 IF Exit_flag THEN PAUSE

3750 IF Exit_flag THEN Exit

3753 ! Inck: $\mathrm{K}=\mathrm{K}+1$

3756 ! IF $K>$ Nfreqs THEN Record

3759 ! IF Omitf(K) THEN PRINT Omitf(K)

3762 ! GOTO Inck

3763 Record: IF G1=1 THEN To_file\$="CRST3"

3764 


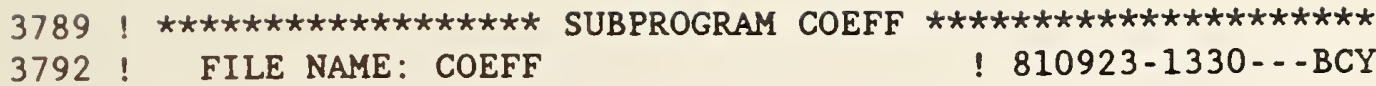

S Calres(

\begin{tabular}{|c|c|c|c|c|c|c|c|}
\hline Aver( & 3624 & 3727 & & & & & \\
\hline $\mathrm{Ca} \$$ & $\begin{array}{l}3612 \\
3660\end{array}$ & $\begin{array}{l}3625 \\
3740\end{array}$ & 3628 & 3629 & 3636 & 3637 & 3644 \\
\hline $\mathrm{Cal}$ & $\begin{array}{l}3612 \\
3774\end{array}$ & $\begin{array}{l}3625 \\
3777\end{array}$ & $\begin{array}{l}3766 \\
3778\end{array}$ & $\begin{array}{l}3767 \\
3778\end{array}$ & $\begin{array}{l}3768 \\
3779\end{array}$ & 3769 & 3773 \\
\hline Check & 3662 & 3666 & & & & & \\
\hline CnnctS & 3641 & 3646 & 3727 & 3764 & & & \\
\hline$D($ & 3618 & 3717 & 3727 & & & & \\
\hline Date\$ & 3618 & 3641 & 3646 & 3678 & 3727 & 3764 & \\
\hline Dim & 3612 & 3621 & & & & & \\
\hline Done: & 3735 & 3687 & & & & & \\
\hline Dsk_nam\$ & 3621 & 3659 & 3727 & & & & \\
\hline Errent & 3727 & 3742 & & & & & \\
\hline Error_flag & 3727 & 3738 & & & & & \\
\hline Exit: & 3781 & 3750 & & & & & \\
\hline Exit_flag & 3742 & 3744 & 3747 & 3750 & & & \\
\hline F1S & 3633 & & & & & & \\
\hline F_oh_file\$ & 3629 & 3637 & 3641 & & & & \\
\hline F_oh_files: & 0 & 3637 & & & & & \\
\hline Fghz & 3723 & 3727 & & & & & \\
\hline Files & 3621 & & & & & & \\
\hline $\mathrm{Fr}$ & 3648 & 3653 & 3653 & 3655 & 3655 & 3658 & \\
\hline Fr_files & 3644 & 3645 & 3646 & 3647 & & & \\
\hline Freq & 3626 & 3684 & 3684 & 3687 & 3717 & 3723 & 3727 \\
\hline Freqs( & 3621 & 3641 & 3646 & 3723 & 3764 & & \\
\hline From_files & 3628 & 3636 & 3642 & 3675 & 3727 & & \\
\hline
\end{tabular}


From_files:

$0 \quad 3636$

G1

$3612 \quad 3643$

$3767 \quad 3768$

$3727 \quad 3740$

$3741 \quad 3763$

3766

Hi_lo_port

$3612 \quad 3727$

I

$\begin{array}{llllll}3699 & 3702 & 3702 & 3705 & 3717 & 3717\end{array}$

Id_cont\$

$\begin{array}{lllll}3618 & 3641 & 3646 & 3681 & 3764\end{array}$

Incfrq:

$3684 \quad 3696$

Inc1:

$3702 \quad 3714$

Incm:

$3711 \quad 3720$

Incr:

$3693 \quad 3729$

Max_pts

$3612 \quad 3764$

Mnt

$3708 \quad 3711$

3711

$\begin{array}{lll}3714 & 3717 & 3717\end{array}$

Nfreqs

$\begin{array}{lllllll}3612 & 3621 & 3621 & 3624 & 3624 & 3624 & 3641\end{array}$

$\begin{array}{lllllll}3646 & 3647 & 3648 & 3687 & 3742 & 3764 & 3765\end{array}$

Omitf(

3624

P

$\begin{array}{llllll}3650 & 3653 & 3653 & 3655 & 3655 & 3656\end{array}$

Power(

$3621 \quad 3642 \quad 3717$

$\operatorname{Prt} \$$

$3612 \quad 3727$

$\operatorname{Rd}($

$\begin{array}{llll}3624 & 3653 & 3655 & 3727\end{array}$

Record:

$3763 \quad 3741$

Repeat

$3690 \quad 3693$

$\begin{array}{llll}3693 & 3696 \quad 3717 & 3727\end{array}$

Repeats

$\begin{array}{lllllll}3612 & 3621 & 3641 & 3646 & 3696 & 3727 & 3764\end{array}$

Result(

$\begin{array}{llllll}3624 & 3647 & 3653 & 3655 & 3727 & 3765\end{array}$

$\mathrm{Sp}$

$\begin{array}{lllllll}3612 & 3641 & 3646 & 3649 & 3652 & 3653 & 3655\end{array}$

$3657 \quad 3727 \quad 3764$

Std

$\begin{array}{llll}3612 & 3641 & 3646 & 3764\end{array}$

Subr :

$3723 \quad 3705$

Time\$

$\begin{array}{llllll}3618 & 3641 & 3646 & 3678 & 3727 & 3764\end{array}$

To_files

$\begin{array}{llllll}3660 & 3740 & 3740 & 3763 & 3764 & 3765\end{array}$ 


$\begin{array}{lrrrrr}\text { To_files: } & 0 & 3763 & & & \\ \text { Typ } & 3632 & 3633 & 3727 & & \\ \text { Typ\$ } & 3612 & 3632 & 3641 & 3646 & 3764 \\ \text { Var( } & 3624 & 3727 & & & \\ \text { Xerr: } & 0 & 3661 & & & \end{array}$


3795 SUB Coeff(Freq, Fghz, Repeat, Repeats, Date $\$$, Time $\$$, Typ, Sp,

From_file\$,Dsk_nam\$, Hiloport, P\$, Cnnct\$, Errcnt,

Error_flag, D(*), Result(*), Aver(*), Var(*), Gl, Rd(*))

3798 OPTION BASE 1

3801 DIM Wrspt $(2,12), \operatorname{Stds}(14,2), \operatorname{Rs}(2,12), A(87), \operatorname{Sdev}(87), S(6)$, C3 $(4,2)$, Pthru $(4,8)$

3804 DIM P1ine $(4,8), \operatorname{Ppad}(4,8)$

3807 ASSIGN \#9 TO "T_RSLT:F8, 1"

3810 IF Repeat $=1$ THEN MAT Aver $=$ ZER

3813 IF Repeat=1 THEN MAT Var=ZER

3816 MAT Rs=ZER

3819 Date=VAL(Date\$)

3822 Time=VAL(Time $\$$ )

3825 Opt\$=VAL\$ (POS (Cnnct\$, "N"))

3828 IF Opt\$ $\bigcirc$ "2" THEN Calib

3831 Opt\$=Opt\$\&Cnnct\$ [5]

3834

3835 Calib: IF $G 1=1$ THEN Cal $=1$

3837

3840

3843

3846

3849

3852

3855

3858

3861

3864

3867

3870

3873

3876

3879

3882

3885

3888

3891

3894

3897

3900

3903

3906

3909

3912

3915

3918

3921

3924

3927

CALL Calib(D(*),Wrspt(*),Stds(*), Opt\$,Error_flag,Rs(*), Hiloport, G1, Rd(*), Freq)

IF Error_flag THEN PRINT CHR\$(7); "FATAL ERROR FOR ";

Freqs (Freq);" GHz"; LIN(1)

IF Error_flag THEN Errcnt=Errent+1

IF Error_flag THEN Omitf(Freq) $=$ Fghz

IF Error_flag THEN SUBEXIT

FOR $I=1$ TO 4

FOR $\mathrm{J}=1$ TO 8

$\operatorname{Pthru}(I, J)=D(I+4, J)$

Pline $(I, J)=D(I+8, J)$

$\operatorname{Ppad}(I, J)=D(I+12, J)$

NEXT J

NEXT I

CALL Rhothu(Wrspt(*), Pthru(*), C3(*))

FOR $I=1$ TO 3

FOR $\mathrm{J}=1$ TO 2

NEXT J

$S(2 * I+J-2)=S t d s(I+7, J)$

NEXT I

CALL Rho2pt(S(*),C3(*), Wrspt(*), Ppad(*))

$\operatorname{Stds}(9,2)=S(4)$

MAT $S=Z E R$

$S(3)=\operatorname{Stds}(3,1)$

$S(4)=\operatorname{Stds}(3,2)$

CALL Rho2pt(S(*),C3(*), Wrspt(*), P1ine(*))

$\operatorname{Stds}(3,2)=S(4)$

$\operatorname{Stds}(3,1)=-20 * \operatorname{LGT}(\operatorname{Stds}(3,1))$

$\operatorname{Stds}(9,1)=-20 \times \operatorname{LGT}(\operatorname{Stds}(9,1))$

$\operatorname{Stds}(11,1)=\mathrm{Sp}$

$\operatorname{stds}(11,2)=$ Pincid

$\operatorname{Stds}(12,1)-$ Pstd

$\operatorname{Stds}(12,2)=$ Pnet

Stds $(13,1)=$ Xmnt

Stds $(13,2)=$ Ymnt

Many $=($ Repeats $>1) *$ Repeat

3936

IF POS(P\$,",") THEN PRINTER IS 7,1 
IF POS(PS, "On) THEN PRINTER IS 0

CALL Print(Fghz, Date\$, Time\$, From_file\$,Dsk_nam\$, Stds(*), Wrspt (*), Rs (*), Many, Repeats)

READ \#9, Repeat

PRINT \#9;Wrspt(*),C3(*), Stds(*), Rs(*), Fghz, Date, Time

READ \#9, Repeat

READ \#9;A(*)

IF Repeat $=1$ THEN MAT Aver $=\mathrm{A}$

IF Repeats=1 THEN Prnt

IF Repeat $O 1$ THEN CALL Avg(Aver(*), $\operatorname{Var}(*), A(*)$, Repeat)

IF Repeat $O$ Repeats THEN SUBEXIT

\section{MAT Sdev=SQR (Var)}

PRINT \#9, Repeats 1 ; Aver(*)

READ \#9, Repeats+1

READ \#9; Wrspt (*),C3(*), Stds(*), Rs (*), Fghz, Date, Time

CALL Print(Fghz, Date\$, Time\$, From_file\$,Dsk_nam\$, Stds(*), $\operatorname{Wrspt}(*), \operatorname{Rs}(*),-1, \operatorname{Repe}-\overline{t s})$

3990

CALL Prnt_sdev(Fghz, Date\$, Time\$, From_file\$, Dsk_nam\$, $\operatorname{Sdev}(*), S p)$

3993 Prnt: PRINTER IS 16

$3996 \quad \mathrm{~J}=0$

3999 Incj: $\mathrm{J}-\mathrm{J}+1$

4002 IF $\mathrm{J}>60$ THEN SUBEXIT

4005

4008

$\operatorname{Result}($ Freq, $J)=\operatorname{Aver}(J)$

4011 SUBEND

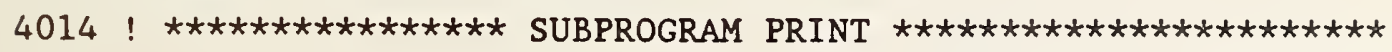
4017

! 820103-1000---BCY\&DHR

S Coeff(

$\begin{array}{lllllll}\text { Al } & 3801 & 3960 & 3963 & 3969 & & \\ \text { Aver( } & 3795 & 3810 & 3963 & 3969 & 3978 & 4005 \\ \text { C3( } & 3801 & 3873 & 3891 & 3906 & 3951 & 3984 \\ \text { Cal } & 3835 & & & & & \end{array}$

Calib: $\quad 3835 \quad 3828$

Cnnct\$ $\quad 3795 \quad 3825 \quad 3831$

DC $\quad \begin{array}{lllll}3795 & 3837 & 3858 & 3861 & 3864\end{array}$

Date $\quad 3819 \quad 3951 \quad 3984$

$\begin{array}{llllll}\text { Date\$ } & 3795 & 3819 & 3945 & 3987 & 3990\end{array}$

Dsk_nam\$ $\quad 3795 \quad 3945 \quad 3987 \quad 3990$

$\begin{array}{llll}\text { Errcnt } & 3795 & 3843 \quad 3843\end{array}$ 


\begin{tabular}{|c|c|c|c|c|c|c|c|}
\hline Error_flag & 3795 & 3837 & 3840 & 3843 & 3846 & 3849 & \\
\hline Fghz & 3795 & 3846 & 3945 & 3951 & 3984 & 3987 & 3990 \\
\hline Freq & 3795 & 3837 & 3840 & 3846 & 4005 & & \\
\hline Freqs ( & 3840 & & & & & & \\
\hline From_files & 3795 & 3945 & 3987 & 3990 & & & \\
\hline G1 & 3795 & 3835 & 3837 & & & & \\
\hline Hiloport & 3795 & 3837 & & & & & \\
\hline I & $\begin{array}{l}3852 \\
3870\end{array}$ & $\begin{array}{l}3858 \\
3876\end{array}$ & $\begin{array}{l}3858 \\
3882\end{array}$ & $\begin{array}{l}3861 \\
3882\end{array}$ & $\begin{array}{l}3861 \\
3888\end{array}$ & $\begin{array}{l}3864 \\
3999\end{array}$ & $\begin{array}{l}3864 \\
4008\end{array}$ \\
\hline $\mathrm{J}$ & $\begin{array}{l}3855 \\
3867 \\
3999\end{array}$ & $\begin{array}{l}3858 \\
3879 \\
4002\end{array}$ & $\begin{array}{l}3858 \\
3882 \\
4005\end{array}$ & $\begin{array}{l}3861 \\
3882 \\
4005\end{array}$ & $\begin{array}{l}3861 \\
3885\end{array}$ & $\begin{array}{l}3864 \\
3996\end{array}$ & $\begin{array}{l}3864 \\
3999\end{array}$ \\
\hline Many & 3936 & 3945 & & & & & \\
\hline Omitf ( & 3846 & & & & & & \\
\hline Opt\$ & 3825 & 3828 & 3831 & 3831 & 3837 & & \\
\hline$P \$$ & 3795 & 3939 & 3942 & & & & \\
\hline Pincid & 3921 & & & & & & \\
\hline Fline ( & 3804 & 3861 & 3906 & & & & \\
\hline Pnet & 3927 & & & & & & \\
\hline Ppad( & 3804 & 3864 & 3891 & & & & \\
\hline Prnt: & 3993 & 3966 & & & & & \\
\hline Pstd & 3924 & & & & & & \\
\hline Pthru( & 3801 & 3858 & 3873 & & & & \\
\hline $\operatorname{Rd}($ & 3795 & 3837 & & & & & \\
\hline Repeat & $\begin{array}{l}3795 \\
3969\end{array}$ & $\begin{array}{l}3810 \\
3969\end{array}$ & $\begin{array}{l}3813 \\
3972\end{array}$ & 3936 & 3948 & 3957 & 3963 \\
\hline Repeats & $\begin{array}{l}3795 \\
3987\end{array}$ & 3936 & 3945 & 3966 & 3972 & 3978 & 3981 \\
\hline Result( & 3795 & 4005 & & & & & \\
\hline $\operatorname{Rs}($ & 3801 & 3816 & 3837 & 3945 & 3951 & 3984 & 3987 \\
\hline
\end{tabular}




\begin{tabular}{|c|c|c|c|c|c|c|c|}
\hline S( & $\begin{array}{l}3801 \\
3906\end{array}$ & $\begin{array}{l}3882 \\
3909\end{array}$ & 3891 & 3894 & 3897 & 3900 & 3903 \\
\hline$S \operatorname{dev}($ & 3801 & 3975 & 3990 & & & & \\
\hline$S_{p}$ & 3795 & 3918 & 3990 & & & & \\
\hline Stds ( & $\begin{array}{l}3801 \\
3912 \\
3927\end{array}$ & $\begin{array}{l}3837 \\
3912 \\
3930\end{array}$ & $\begin{array}{l}3882 \\
3915 \\
3933\end{array}$ & $\begin{array}{l}3894 \\
3915 \\
3945\end{array}$ & $\begin{array}{l}3900 \\
3918 \\
3951\end{array}$ & $\begin{array}{l}3903 \\
3921 \\
3984\end{array}$ & $\begin{array}{l}3909 \\
3924 \\
3987\end{array}$ \\
\hline Time & 3822 & 3951 & 3984 & & & & \\
\hline Time\$ & 3795 & 3822 & 3945 & 3987 & 3990 & & \\
\hline Typ & 3795 & & & & & & \\
\hline $\operatorname{Var}($ & 3795 & 3813 & 3969 & 3975 & & & \\
\hline Wrspt( & $\begin{array}{l}3801 \\
3984\end{array}$ & $\begin{array}{l}3837 \\
3987\end{array}$ & 3873 & 3891 & 3906 & 3945 & 3951 \\
\hline Xmnt & 3930 & & & & & & \\
\hline Ymnt & 933 & & & & & & \\
\hline
\end{tabular}


4020 SUB Print(Fghz,Date\$,Time\$,File\$,Dsk_nam\$,Stds(*), Wrspt(*),

4023 OPTION BASE 1

$$
\text { Rs (*), Rpt_no, Repeat) }
$$

4026 DIM Qpts $(2,12)$

4029 IF Rpt_no=0 THEN PRINT "

SIX-PORT

PARAMETER RESULTS "

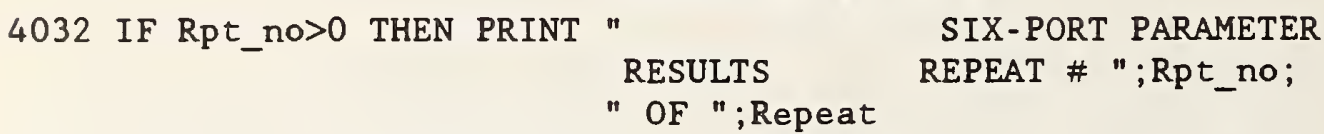

4035 IF RPt_no<0 THEN PRINT " SIX-PORT PARAMETER

RESULTS AVERAGE OF "; Repeat;

" REPEATS"

4038 PRINT LIN(1),"

4041 PRINT "YEAR MONTH DAY TIME FILE NAME DISK IDENT FREQ. IN $\mathrm{GHz}$ "

4044 PRINT USING Head20; Date $[1,2]$, Date $\$[3,4]$, Date $\$[5,6]$, Time\$, File\$, Dsk_nam\$, Fghz

4047 Head20: IMAGE $\mathrm{X}, 2 \mathrm{~A}, 4 \mathrm{X}, 2 \mathrm{~A}, 5 \mathrm{X}, 2 \mathrm{~A}, 7 \mathrm{X}, 6 \mathrm{~A}, 6 \mathrm{X}, 6 \mathrm{~A}, 7 \mathrm{X}, 7 \mathrm{~A}, 7 \mathrm{X}, \mathrm{DZ}, 6 \mathrm{D}$

4050 PRINT LIN(1)," SCATTERING PARAMETERS OF STANDARDS "

4053 PRINT

4056 PRINT"

MAG--S11--ANG dB--S12--ANG

MAG--S22--ANG "

4059 Head51:IMAGE 11A, 4X, Z.6D, 2X, 4D .3D, X, 3D.6D, 5D . 3D , 4X, Z . 6D , 2X, 4D. 3D

4062 PRINT USING Head51;"2-PORT ", $\operatorname{Stds}(8,1), \operatorname{stds}(8,2)$, $\operatorname{stds}(9,1), \operatorname{stds}(9,2), \operatorname{stds}(10,1)$,

$\operatorname{Stds}(10,2)$

4065 Head52: IMAGE 11A,25X,Z.6D,5D.3D

4068 PRINT USING Head52;"LINE

4071 Head60: IMAGE 11A, 4X, Z.6D,6D.3D

", Stds $(3,1), \operatorname{stds}(3,2)$

4074

PRINT USING Head60;"SHORT \#1

"; $\operatorname{stds}(4,1), \operatorname{stds}(4,2)$

PRINT USING Head60;"SHORT \#2 ";Stds $(5,1), \operatorname{Stds}(5,2)$

4080 Head70: IMAGE 14A,X,Z.6D,6D.3D

4083

PRINT USING Head70;"OPEN 6-PORT \#1",Stds $(6,1), \operatorname{Stds}(6,2)$

4086

4089 Head75: IMAGE 11A, 2X, 3D.6D, 5D . 3D, 18A, D, 2A

4092 IF Stds $(1,1) \diamond 0$ THEN PRINT USING Head75; "STD MOUNT",Stds $(13,1)$,

$\operatorname{Stds}(13,2), "($ ON 6-PORT \#",Stds $(11,1), ")$ "

4095 Head76: IMAGE /26A, 2X, 2D . 4D, 2X, 11A

4098 Head77: IMAGE 26A, 2X, 2D.4D, 2X, 39A

4101 IF Stds $(11,1) \diamond 0$ THEN PRINT USING Head76;"STD MOUNT POWER:

", $\operatorname{stds}(12,1)$," MILLIWATTS"

4104 IF Stds $(11,1) \diamond 0$ THEN PRINT USING Head77;"STD MOUNT INCIDENT

POWER: ", Stds $(11,2)$, " MILLIWATTS"

4107 Head78: IMAGE /46A, X, Z.4D, 20A, X, Z.4D

4110 IF Stds $(11,1)=0$ THEN PRINT USING Head78; "POWER RATIO CONSTANTS:

6-PORT \#1: (K1)=",Wrspt $(1,12), " \quad 6-\mathrm{PORT} \# 2:(\mathrm{K} 2)="$,

Wrspt $(2,12)$

4113 IF Stds $(11,1) \diamond 0$ THEN PRINT USING Head78; "POWER STANDARD CONSTANTS: 6-PORT

\#1: $(\mathrm{K} 1)=", \operatorname{Wrspt}(1,12), " \quad 6-\operatorname{PORT} \# 2:(\mathrm{K} 2)=", \operatorname{Wrspt}(2,12)$

4116

PRINT

GOSUB Qprint

4119
4122

PRINT " 
4125 PRINT "RMS PARABOLOID RES*1E6 | *** CIRCLE RESIDUALS*1E6 $* \star * \mid 2$-PORT RES*1E6"

4128 PRINT " INITIAL FINAL| SHORT OPEN THRU LINE 2PORT AVG I THRU LINE 2-PORT"

4131 MAT Rs-Rs*(1E6)

4134 Head_res: IMAGE 9A, X,2(6D), X, A, 4(5D,X), 2X, 2(5D), 2A, 3(5D, X)

4137 Head resl: IMAGE 9A, $X, 2(6 D), X, A, 4(5 D, X), 2 X, 2(5 D), 2 A$

4140 PRINT USING Head res;"6-PORT \#1", Rs $(1,1) *(\operatorname{Rs}(1,2) / 1 E 6), \operatorname{Rs}(1,1)$, "|", $\operatorname{Rs}(1,3), \operatorname{Rs}(1,4), \operatorname{Rs}(1,5), \operatorname{Rs}(1,6), \operatorname{Rs}(1,7), \operatorname{Rs}(1,8), " \mid "$, $\operatorname{Rs}(1,9), \operatorname{Rs}(1,10), \operatorname{Rs}(1,11)$

4143 PRINT USING Head_res1; "6-PORT \#2", Rs $(2,1) *(\operatorname{Rs}(2,2) / 1 E 6), \operatorname{Rs}(2,1)$, $"|", \operatorname{Rs}(2,3), \operatorname{Rs}(2,4), \operatorname{Rs}(2,5), \operatorname{Rs}(2,6), \operatorname{Rs}(2,7), \operatorname{Rs}(2,8), "| "$

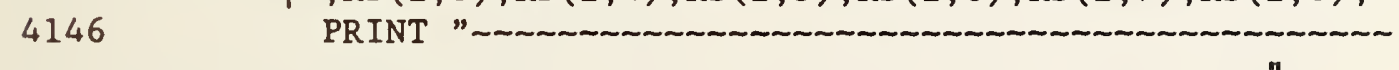

S Print(

\begin{tabular}{|c|c|c|c|}
\hline Date\$ & 4020 & 4044 & 4044 \\
\hline Dsk_nam\$ & 4020 & 4044 & \\
\hline Fghz & 4020 & 4044 & \\
\hline File\$ & 4020 & 4044 & \\
\hline Head20: & 4047 & & \\
\hline Head51: & 4059 & & \\
\hline Head52: & 4065 & & \\
\hline Head60: & 4071 & & \\
\hline Head70: & 4080 & & \\
\hline Head75: & 4089 & & \\
\hline
\end{tabular}




\begin{tabular}{|c|c|c|c|c|c|c|c|}
\hline Head76: & 4095 & & & & & & \\
\hline Head77: & 4098 & & & & & & \\
\hline Head78: & 4107 & & & & & & \\
\hline Head_resl: & 4137 & & & & & & \\
\hline Head_res: & 4134 & & & & & & \\
\hline Qprint: & 4155 & 4119 & & & & & \\
\hline Qpts ( & $\begin{array}{l}4026 \\
4173 \\
4176\end{array}$ & $\begin{array}{l}4158 \\
4173 \\
4176\end{array}$ & $\begin{array}{l}4173 \\
4173 \\
4176\end{array}$ & $\begin{array}{l}4173 \\
4176 \\
4176\end{array}$ & $\begin{array}{l}4173 \\
4176\end{array}$ & $\begin{array}{l}4173 \\
4176\end{array}$ & $\begin{array}{l}4173 \\
4176\end{array}$ \\
\hline Qpts1: & 4170 & & & & & & \\
\hline Qpts 2: & 4179 & & & & & & \\
\hline Repeat & 4020 & 4032 & 4035 & & & & \\
\hline Rpt_no & 4020 & 4029 & 4032 & 4032 & 4035 & & \\
\hline Rs ( & $\begin{array}{l}4020 \\
4140 \\
4140 \\
4143\end{array}$ & $\begin{array}{l}4131 \\
4140 \\
4143 \\
4143\end{array}$ & $\begin{array}{l}4131 \\
4140 \\
4143 \\
4143\end{array}$ & $\begin{array}{l}4140 \\
4140 \\
4143 \\
4149\end{array}$ & $\begin{array}{l}4140 \\
4140 \\
4143 \\
4149\end{array}$ & $\begin{array}{l}4140 \\
4140 \\
4143\end{array}$ & $\begin{array}{l}4140 \\
4140 \\
4143\end{array}$ \\
\hline Stds ( & $\begin{array}{l}4020 \\
4068 \\
4083 \\
4101\end{array}$ & $\begin{array}{l}4062 \\
4068 \\
4086 \\
4101\end{array}$ & $\begin{array}{l}4062 \\
4074 \\
4086 \\
4104\end{array}$ & $\begin{array}{l}4062 \\
4074 \\
4092 \\
4104\end{array}$ & $\begin{array}{l}4062 \\
4077 \\
4092 \\
4110\end{array}$ & $\begin{array}{l}4062 \\
4077 \\
4092 \\
4113\end{array}$ & $\begin{array}{l}4062 \\
4083 \\
4092\end{array}$ \\
\hline Time\$ & 4020 & 4044 & & & & & \\
\hline Wrspt ( & 4020 & 4110 & 4110 & 4113 & 4113 & 4158 & \\
\hline
\end{tabular}


4194 SUB Prnt_sdev(Fghz, Date\$,Time\$,File\$,Dsk_nam\$, Sdev(*), Sp)

4197 OPTION BASE 1

4200 PRINT LIN(2);" STANDARD DEVIATIONS OF SELECTED PARAMETER RESULTS"

4203 PRINT LIN(1);"

4206 PRINT "YEAR MONTH DAY TIME FILE NAME DISK IDENT FREQ. IN GHz"

4209 PRINT USING Head2; Date $[1,2]$, Date $\$[3,4]$, Date $\$[5,6]$, Time\$, File\$, Dsk_nam\$, Fghz

4212 Head2: IMAGE X, 2A, 4X, 2A, 5X, 2A, 7X, 6A, 6X, 6A, 7X, 7A, 7X, DZ . 6D

4215 PRINT LIN(1)," STANDARDS "

4218 PRINT

4221 PRINT "

$$
\begin{aligned}
& \text { MAG--S11 dB--S12 } \\
& \text { MAG--S22" }
\end{aligned}
$$

4224 Head3: IMAGE 11A, 7X, Z.6D,12X, Z.6D, 14X, Z.6D

4227 PRINT USING Head3;"2-PORT ", Sdev(37), Sdev(39), $\operatorname{Sdev}(41)$

4230 Head4: IMAGE 11A,27X, 2.6D

4233 PRINT USING Head4;"LINE ",Sdev(29)

4236 Head5: IMAGE 15A,3X, 2.6D

4239 PRINT USING Head5;"SHORT ",Sdev(31)

4242 PRINT USING Head5;"OPEN 6-PORT \#1 ",Sdev(33)

4245 PRINT USING Head5;"OPEN 6-PORT \#2 ",Sdev(35)

4248 Head7: IMAGE 11A, 8X, .6D,8X,17A, D, 2A

4251 IF SP $>0$ THEN PRINT USING Head7; "STD MOUNT ",Sdev(49),

4254 Head8: IMAGE /26A, 4X, .4D, 2X, 11A

4257 Head9: IMAGE 26A, 4X, .4D, 2X, 39A

4260 IF SP $>0$ THEN PRINT USING Head8; "STD MOUNT POWER: ",

4263 IF SP $>0$ THEN PRINT USING Head9;"STD MOUNT INCIDENT POWER: ", Sdev(36), " MILIWATTS"

4266 Head10: IMAGE /46A, X, Z.4D, 19A, X, Z.4D

4269 IF $S P=0$ THEN PRINT USING Headl0; "POWER RATIO CONSTANTS:

6-PORT \#1: $(\mathrm{K} 1)=", \operatorname{Sdev}(12), "$

6-PORT \#2: (K2)=", $\operatorname{Sdev}(24)$

4272 IF SP $>0$ THEN PRINT USING Head10; "POWER CALIBRATION CONSTANTS: 6 -PORT \#1: $(\mathrm{K} 1)=", \operatorname{Sdev}(12), "$

4275 PRINT CHR\$(12)

6-PORT \#2: (K2)=", Sdev(24)

4278 SUBEND

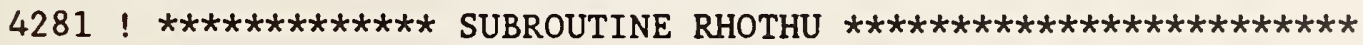

4284 ! FILE NAME: RHOTHU

! 830310-1100---BCY

S Prnt_sdev(

Date\$ $\quad 4194 \quad 4209 \quad 4209 \quad 4209$

Dsk_nams $\quad 4194 \quad 4209$

Fghz $\quad 4194 \quad 4209$ 


\begin{tabular}{|c|c|c|c|c|c|c|c|}
\hline File\$ & 4194 & 4209 & & & & & \\
\hline Head10: & 4266 & & & & & & \\
\hline Head2: & 4212 & & & & & & \\
\hline Head3: & 4224 & & & & & & \\
\hline Head4: & 4230 & & & & & & \\
\hline Head5: & 4236 & & & & & & \\
\hline Head7: & 4248 & & & & & & \\
\hline Head8: & 4254 & & & & & & \\
\hline Head9: & 4257 & & & & & & \\
\hline Sdev( & $\begin{array}{l}4194 \\
4245 \\
4272\end{array}$ & $\begin{array}{l}4227 \\
4251\end{array}$ & $\begin{array}{l}4227 \\
4260\end{array}$ & $\begin{array}{l}4227 \\
4263\end{array}$ & $\begin{array}{l}4233 \\
4269\end{array}$ & $\begin{array}{l}4239 \\
4269\end{array}$ & $\begin{array}{l}4242 \\
4272\end{array}$ \\
\hline $\mathrm{Sp}$ & 4194 & 4251 & 4251 & 4260 & 4263 & 4269 & 4272 \\
\hline Timeș & 4194 & 4209 & & & & & \\
\hline
\end{tabular}


4287 SUB Rhothu(Wrspt(*), Pthru(*), C3(*))

4290 OPTION BASE 1

4293 DIM W $(4,8), \operatorname{Rthru}(4,4)$

4296 DEG

4299 FOR I=1 TO 4

4302 CALL W(Pthru(*), 1,4,0,1,Wrspt(*),W(*))

4305 CALL Wg(Wrspt (*), 1, 0,W(I, 1),W(I, 2), Rthru(I, 1), Rthru(I , 2))

$4308 \quad \operatorname{C3}(I, 2)=\operatorname{Argth}=\operatorname{ATN}(\operatorname{Rthru}(I, 2) /(\operatorname{Rthru}(I, 1)+1 E-20))+180 *$ (Rthru $(I, 1)<0) * S G N(\operatorname{Rthru}(I, 2)+1 E-20)$

4311 C3 $(I, 1)-\operatorname{Magth}=\operatorname{SQR}(\operatorname{Rthru}(I, 1) \wedge 2+\operatorname{Rthru}(I, 2) \wedge 2)$

4314 NEXT I

4317 SUBEND

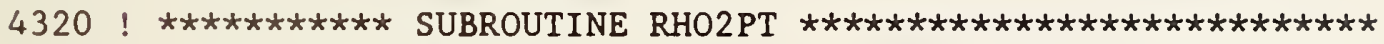

4323 ! FILE NAME: RHO2PT

! 830311-1130---BCY

S Rhothu(

$\begin{array}{llllllll}\text { Argth } & 4308 & & & & & & \\ \text { C3( } & 4287 & 4308 & 4311 & & & & \\ \text { I } & 4299 & 4305 & 4305 & 4305 & 4305 & 4308 & 4308 \\ & 4308 & 4308 & 4308 & 4311 & 4311 & 4311 & 4314 \\ \text { Magth } & 4311 & & & & & & \\ \text { Pthru( } & 4287 & 4302 & & & & & \\ \text { Rthru( } & 4293 & 4305 & 4305 & 4308 & 4308 & 4308 & 4308 \\ & 4311 & 4311 & & & & & \\ \text { Wi } & 4293 & 4302 & 4305 & 4305 & & & \\ \text { Wrspt } & 4287 & 4302 & 4305 & & & & \end{array}$


4326 SUB Rho2pt(S(*),C3(*),Wrspt(*), P2pt(*))

4329 OPTION BASE 1

4332 DIM $G(4,4), W(4,8)$

4335 DEG

$4338 \quad I=1$

4341 Argth $=\mathrm{C} 3(\mathrm{I}, 2)$

4344 CALL W(P2pt(*), 1,4,0,1,Wrspt(*),W(*))

4347 CALL Wg(Wrspt(*), 1,0,W(I,1),W(I,2), G(I,1),G(I,2))

$4350 \quad$ CALL Rect(S(1), S(2),XI,YI)

$4353 \quad \operatorname{CALL} \operatorname{Csub}(G(I, 1), G(I, 2), X 1, Y 1, X, Y)$

$4356 \quad \operatorname{Arg} 2 \mathrm{P}=\mathrm{ATN}(\mathrm{Y} /(\mathrm{X}+1 \mathrm{E}-20))+180 *(\mathrm{X}<0) * \mathrm{SGN}(\mathrm{Y}+1 \mathrm{E}-20)$

4359 Arg12=Arg2p-Argth

4362 Test: IF ABS(Arg12)<180 THEN Skp

$4365 \quad$ Arg12-Arg12-SGN(Arg12) $* 360$

4368 GOTO Test

4371 Skp: Args4=S(4)

4374 Sgn12-SGN (Arg12)*1

4377 Sgns $4=$ SGN (Args 4$) * 1$

4380 IF Sgn12=Sgns 4 THEN Subt

4383 Args4=Args4-SGN(Args4) $* 180$

4386 IF ABS(Arg12-Args4) $<90$ THEN Switch

4389 Subt: IF ABS(Arg12-Args 4)<90 THEN Exit

4392 Switch: $S(4)=S(4)-\operatorname{SGN}(S(4)) * 180$

4395 Exit:SUBEND

4398 ! $* * * * * * * * *$ SUBPROGRAM DISC COPY $* * * * * * * * * * * * * * * * *$

4401 ! FILE NAME: DCOPY ! 820116-0110---BCY

S Rho2pt(

$\begin{array}{llllllll}\text { Arg12 } & 4359 & 4362 & 4365 & 4365 & 4365 & 4374 & 4386 \\ & 4389 & & & & & & \\ \text { Arg2p } & 4356 & 4359 & & & & & \\ \text { Args4 } & 4371 & 4377 & 4383 & 4383 & 4383 & 4386 & 4389 \\ \text { Argth } & 4341 & 4359 & & & & & \\ \text { C3( } & 4326 & 4341 & & & & & \\ \text { Exit: } & 4395 & 4389 & & & & & \\ \text { G( } & 4332 & 4347 & 4347 & 4353 & 4353 & & \\ \text { I } & 4338 & 4341 & 4347 & 4347 & 4347 & 4347 & 4353 \\ \text { P2pt( } & 4353 & & & & & & \\ \text { S( } & 4326 & 4344 & & & & & \\ \text { Sgn12 } & 4326 & 4350 & 4350 & 4371 & 4392 & 4392 & 4392 \\ \text { Sgns4 } & 4374 & 4380 & & & & & \\ & 4377 & 4380 & & & & & \end{array}$




$\begin{array}{lllll}\text { Skp: } & 4371 & 4362 & & \\ \text { Subt: } & 4389 & 4380 & & \\ \text { Switch: } & 4392 & 4386 & & \\ \text { Test: } & 4362 & 4368 & & \\ \text { W( } & 4332 & 4344 & 4347 & 4347 \\ \text { Wrspt } & 4326 & 4344 & 4347 & \\ \text { X } & 4353 & 4356 & 4356 & \\ \text { XI } & 4350 & 4353 & & \\ \text { Y } & 4353 & 4356 & 4356 & \\ \text { Y1 } & 4350 & 4353 & \end{array}$


4404 SUB Disc_copy(Fil_nam\$,Nfreqs, Typ\$, Ca\$)

4407 OPTION BASE 1

4410 DIM Date\$[6], Time\$[6]

4413 DIM Freqs(Nfreqs), Id_content\$[80], Result(Nfreqs, 52)

4416 F1S-VALS (POS (Typ\$, "P") +1)

4419 CALL Redhed("CRST" \&Ca\$, " :F8, 1", Date\$, Time\$, Typ\$, Nfreqs,

Freqs (*), Repeats, Sp, Std, Id_content\$, Cnnct\$)

4422 CALL Redwrt("CRST"\&CaS, ":F8,1"," ", Resuīt(*), Nfreqs)

4425 CALL Wrthed(Fil_nam\$,":F8,0", Nfreqs, Date\$,Time\$, Typ\$, Nfréqs, Freqs (*), Repeats, Sp,Std,Id_content\$, Cnnct\$)

4428 CALL Redwrt(Fil_nam\$," ", ":F8,0",Result(*), Nfreqs)

4431 SUBEND

4434 ! $* * * * * * * * * * * * * * *$ SUBPROGRAM DISC TRANS $* * * * * * * * * * * * * * * * *$

4437 ! FILE NAME: DTRANS

! 820116-0120 - - - BCY

S Disc_copy(

\begin{tabular}{|c|c|c|c|c|c|c|c|}
\hline $\mathrm{CaS}$ & 4404 & 4419 & 4422 & & & & \\
\hline Cnncts & 4419 & 4425 & & & & & \\
\hline Date\$ & 4410 & 4419 & 4425 & & & & \\
\hline F1S & 4416 & & & & & & \\
\hline Fil_nams & 4404 & 4425 & 4428 & & & & \\
\hline Freqs ( & 4413 & 4419 & 4425 & & & & \\
\hline Id_contents & 4413 & 4419 & 4425 & & & & \\
\hline Nfreqs & $\begin{array}{l}4404 \\
4428\end{array}$ & 4413 & 4413 & 4419 & 4422 & 4425 & 4425 \\
\hline Repeats & 4419 & 4425 & & & & & \\
\hline Result( & 4413 & 4422 & 4428 & & & & \\
\hline $\mathrm{Sp}$ & 4419 & 4425 & & & & & \\
\hline Std & 4419 & 4425 & & & & & \\
\hline Times & 4410 & 4419 & 4425 & & & & \\
\hline Typ\$ & 4404 & 4416 & 4419 & 4425 & & & \\
\hline
\end{tabular}




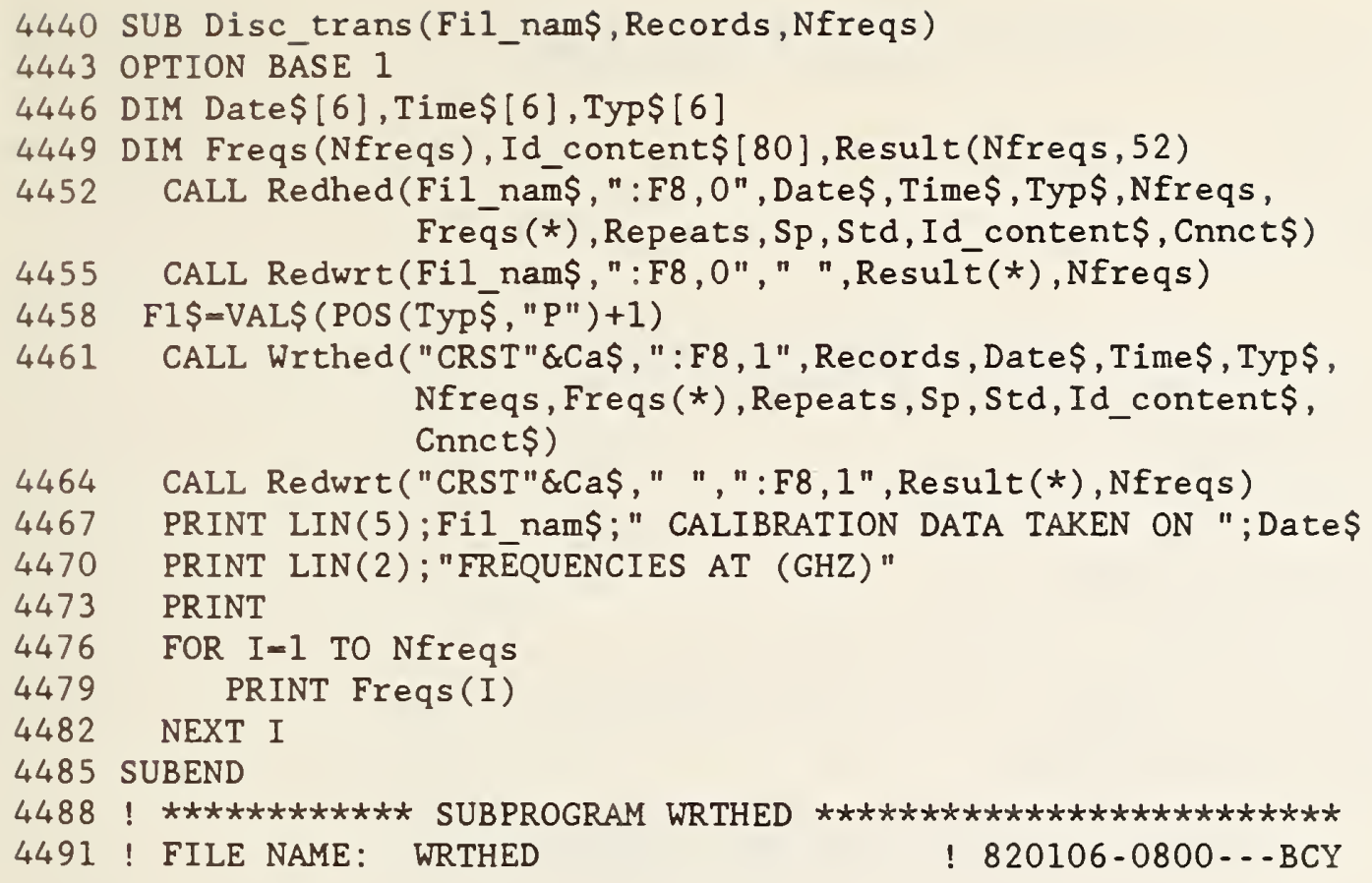


4494 SUB Wrthed(File\$,Msu\$, Records, Date\$, Time\$,Typ\$,Nfreqs, Freq_o(*), Repeats, Sp, Std_mnt, Id_content\$,

4497 OPTION BASE 1 Cnnct )

4500 DIM IdS [19], Overhead\$ [80]

4503 MASS STORAGE IS MsuS

4504 PRINT File\$

4505 WAIT 2500

4506 OverheadS="R2: 9 strings. R3: 4 strings, 4 real. R4:

2 strings [80]. R5\&R6: array (Nfreqs)"

4509 IdŞ="IDENTIFIER STRING:

4512 Retry: DISP " "

4515 PRINT CHR\$ (12)

4518 ! ON ERROR GOTO TraP

4521 ASSIGN \#1 TO File\$, Check

4524 ASSIGN * TO \#1

4527

4530

4533

IF Check THEN CREATE File\$, $6+2 *$ Records ASSIGN \#1 TO Files

PRINT \#1,1;Overhead\$

4536

4539

4542

4545

4548

4551

4554

4557 SUBEXIT

4560 Trap: OFF ERROR

4563

4566 SUBEND

PRINT \#1,2; "DATE\$", "TIME\$", "TYP\$", "CNNCT", "NFREQS",

"REPEATS", "6-PORT" , "STD MNT" , "FREQUENCIES"

PRINT \#1,3;Date\$, Time\$, Typ\$, Cnnct\$, Nfreqs, Repeats, Sp, Std_mnt

4569 ! $* * * * * * * * * * * *$ SUBPROGRAM REDHED

4572 ! FILE NAME: REDHED

! 820106-0730---BCY

S Wrthed(

$\begin{array}{llllll}\text { Check } & 4521 & 4527 & & \\ \text { Cnnct\$ } & 4494 & 4539 & & & \\ \text { Date\$ } & 4494 & 4539 & & & \\ \text { File\$ } & 4494 & 4504 & 4521 & 4527 & 4530 \\ \text { Freq_o( } & 4494 & 4548 & & & \\ \text { Id\$ } & 4500 & 4509 & 4542 & & \\ \text { Id_content\$ } & 4494 & 4542 & & \\ \text { Msu\$ } & 4494 & 4503 & \\ \text { Nfreqs } & 4494 & 4539 & \end{array}$




\begin{tabular}{|c|c|c|}
\hline Overhead\$ & 4500 & 4506 \\
\hline Records & 4494 & 4527 \\
\hline Repeats & 4494 & 4539 \\
\hline Retry: & 4512 & 4563 \\
\hline $\mathrm{Sp}$ & 4494 & 4539 \\
\hline Std_mnt & 4494 & 4539 \\
\hline Times & 4494 & 4539 \\
\hline Trap: & 4560 & \\
\hline Typ\$ & 4494 & 4539 \\
\hline
\end{tabular}


4575 SUB Redhed(File\$,Msu\$, Date\$, Time\$, Typ\$, Nfreqs, Freq_o(*),

4578 OPTION BASE 1

Repeats,Sp,Std_mnt,Id_content\$,Cnnct\$)

4581 DIM Id\$[19], Overhead\$ [80], Form\$(8) [11]

4584 MASS STORAGE IS MsuS

4585 PRINT File\$

4586 WAIT 2500

4587 Retry: DISP " "

4590

PRINT CHR\$(12)

4593 !

4596

ON ERROR GOTO Trap

4599

ASSIGN \#1 TO File\$

4602

READ \#1,1;Overhead\$

4605

READ \#1,2; Form\$(*)

READ \#1,3; Date\$, Time\$, Typ\$, Cnnct\$, Nfreqs, Repeats, $\mathrm{Sp}, \mathrm{Std}$ mnt

4608

4611

4614

4617

4620

4623 SUBEXIT

4626 Trap: OFF ERROR

4627 PRINT Files\$

4629 IF FNDerr(ERRM\$) THEN Retry

4632 SUBEND

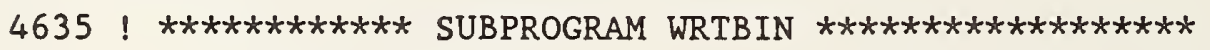

4638 ! FILE NAME: WRTBIN

! 811217-1500---BCY

S Redhed(

$\begin{array}{llll}\text { Cnnct\$ } & 4575 & 4605 \\ \text { Date\$ } & 4575 & 4605 & \\ \text { File\$ } & 4575 & 4585 & 4596 \\ \text { Files\$ } & 4627 & & \\ \text { Form\$ } & 4581 & 4602 \\ \text { Freq_o( } & 4575 & 4614 \\ \text { Id\$ } & 4581 & 4608 \\ \text { Id_content\$ } & 4575 & 4608 \\ \text { MsuS } & 4575 & 4584 \\ \text { Nfreqs } & 4575 & 4605 \\ \text { Overhead\$ } & 4581 & 4599 \\ \text { Repeats } & 4575 & 4605\end{array}$




$\begin{array}{lll}\text { Retry: } & 4587 & 4629 \\ \text { Sp } & 4575 & 4605 \\ \text { Std_mnt } & 4575 & 4605 \\ \text { Time\$ } & 4575 & 4605 \\ \text { Trap: } & 4626 & \\ \text { Typ\$ } & 4575 & 4605\end{array}$


4641 SUB Wrtbin(File\$,MsuS,File(*), Records)

4644 OPTION BASE 1

4647 MASS STORAGE IS Msu\$

4650 Retry: DISP " "

4653

4656

4659

PRINT CHRS(12)

ON ERROR GOTO Trap

FCREATE File $\$$, Records

4662 Fprint: FPRINT File\$, File(*)

4665

OFF ERROR

4668 SUBEXIT

4671 Trap: OFF ERROR

4674

IF ERRN $=54$ THEN Fprint

4677

4680 SUBEND

4683 ! ***************** SUBPROGRAM REDWRT $* * * * * * * * * * * * * * * * * * * * * * *$ 4686 ! FILE NAME: REDWRT

! 820116-1230---BCY

S Wrtbinc

\begin{tabular}{|c|c|c|}
\hline File\$ & 4641 & 4659 \\
\hline File( & 4641 & 4662 \\
\hline Fprint: & 4662 & 4674 \\
\hline Msu\$ & 4641 & 4647 \\
\hline Records & 4641 & 4659 \\
\hline Retry: & 4650 & 4677 \\
\hline Trap: & 4671 & 4656 \\
\hline
\end{tabular}


4689 SUB Redwrt(File\$, From\$, To\$,Data(*), Nfreqs)

4692 OPTION BASE 1

4695 Start: PRINT CHR\$(12)

4698 DISP " "

4701 ! ON ERROR GOTO TraP

4704 ASSIGN \#1 TO File\$

4707 IF TO\$=" " THEN Read

4710 MASS STORAGE IS TO\$

4713 Inci: I-I+1

4716 IF I>Nfreqs THEN Exit

4719 READ \#1,5+2*I

$4722 \quad \mathrm{~J}=0$

4725 Inc $\mathrm{j}: \mathrm{J}=\mathrm{J}+1$

4728 IF $J>52$ THEN Inci

4731 PRINT \#1;Data (I, J )

4734 GOTO Incj

4737 Read: MASS STORAGE IS From\$

4740 Inc_1: $I=I+1$

4743 IF I $>$ Nfreqs THEN Exit

4746 READ \#1, 5+2*I

$4749 \quad \mathrm{~J}=0$

4752 Inc_j: $\mathrm{J}=\mathrm{J}+1$

4755 IF J>52 THEN Inc_i

4758 READ \#1; Data $(I, \bar{J})$

4761 GOTO Inc_j

4764 Exit: ASSI $\overrightarrow{G N}$ * TO \#I

4767 OFF ERROR

4770 SUBEXIT

4773 Trap: OFF ERROR

4776 IF FNDerr(ERRM\$) THEN Start

4779 SUBEND

4782 ! $* * * * * * * * * * *$ FUNCTION FNDERR $* * * * * * * * * * * * * * * *$

4785 ! FILE NAME: FNDERR ! 820127-1745-..BCY

S Redwrt(
Datal
$4689 \quad 4731 \quad 4758$
Exit: $\quad 4764 \quad 4716 \quad 4743$
File\$ $\quad 4689 \quad 4704$
From\$ $\quad 4689 \quad 4737$
I
$\begin{array}{lllllll}4713 & 4713 & 4716 & 4719 & 4731 & 4740 & 4740\end{array}$
$4743 \quad 4746 \quad 4758$
Inc_i: $\quad 4740 \quad 4755$
Inc_j : $\quad 4752 \quad 4761$
Inci: $\quad 4713 \quad 4728$
Incj : $\quad 4725 \quad 4734$ 


$\begin{array}{llllllll}\text { J } & 4722 & 4725 & 4725 & 4728 & 4731 & 4749 & 4752 \\ & 4752 & 4755 & 4758 & & & & \\ \text { Nfreqs } & 4689 & 4716 & 4743 & & & \\ \text { Read: } & 4737 & 4707 & & & & \\ \text { Start: } & 4695 & 4776 & & & \\ \text { To\$ } & 4689 & 4707 & 4710 & & & & \\ \text { Trap: } & 4773 & & & & & & \end{array}$




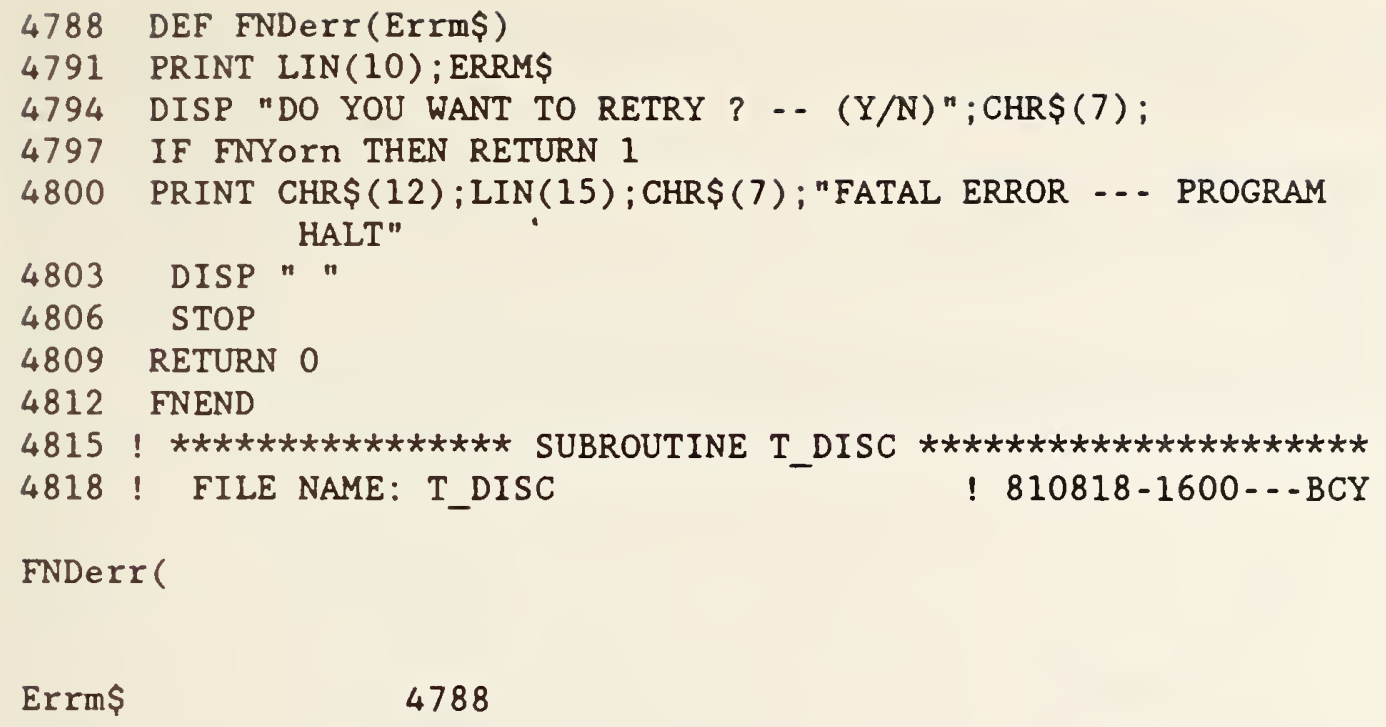


4821 SUB T_disc(File\$,Records, Length)

4824 ON ERROR GOTO Errml

4827 CREATE File\$, Records, Length

4830 SUBEXIT

4833 Errm1: PRINT ERRM\$

4836

4839

PRINT "PAUSE IN EFFECT"

4842

4845

PAUSE

SUBEND

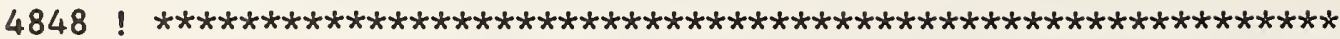

S T_disc(

$\begin{array}{lll}\text { Errml: } & 4833 & 4824 \\ \text { Files } & 4821 & 4827 \\ \text { Length } & 4821 & 4827 \\ \text { Records } & 4821 & 4827\end{array}$




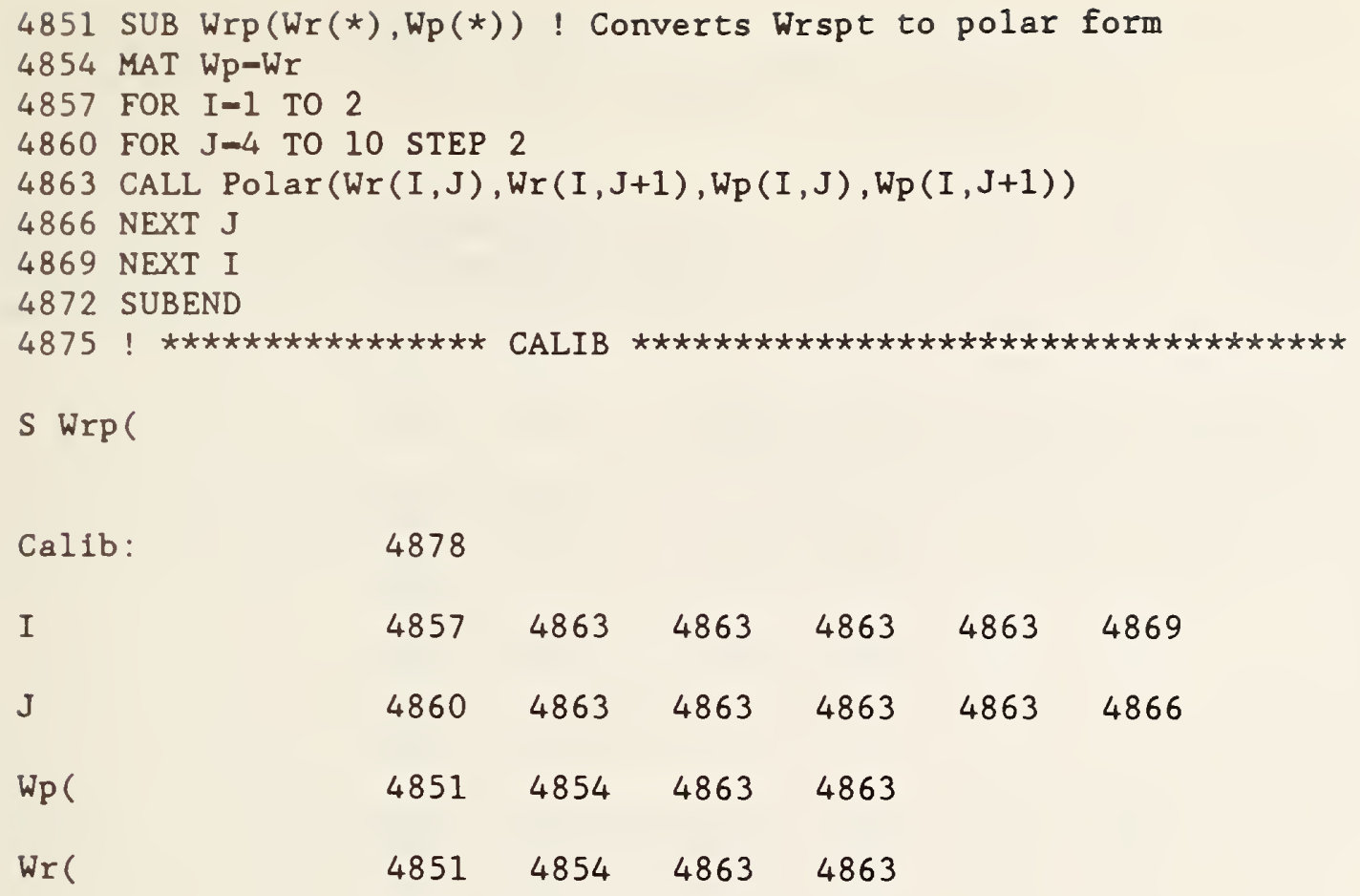


4878 Calib: SUB Calib(Pwr(*),Wrspt(*), Stds(*), Opt\$,Error_flag, Resid(*), Hi_lo_port, G1, Rd(*), Freq)

4881 OPTION BASE 1 ! -...-(81/07/31) -...GFE-GEC-......

4884 DIM V(1:5),W(1:16,1:8), $\operatorname{Res}(2,12)$

4887 MAT Resid=ZER

! Cres is NIOSH CR record 7 p. 5-10

4890 MAT Res=ZER

! Cres is NIOSH

P. $6-24$

4893 Error_flag=0

4896 IF Gl=1 THEN GOTO 4912

4897 FOR $\mathrm{SP}=1$ TO 2

4899 CALL Iparab(Pwr(*),Sp,V(*), Error_flag)

4902 IF Error_flag>0 THEN GOTO Calib_exit

4905 CALL Fparab(Pwr(*),Sp,V(*),Wrspt(*), Res(*),Error_flag,Hi_1o_port)

4908 IF Error_flag $>0$ THEN GOTO Calib_exit

4910 NEXT Sp

4911 GOTO 4921

$4912 !$ CONT

4916 FOR $P=1$ TO 5

4917 FOR $\mathrm{Sp}=1$ TO 2

4918 Wrspt (Sp, P)=Rd (Freq, Sp, P)

4919 NEXT Sp

4920 NEXT $P$

4921 !

4923 ! PRINT $\operatorname{Wrspt}(1,1), \operatorname{Wrspt}(1,2), \operatorname{Wrspt}(1,3), \operatorname{Wrspt}(1,4)$, Wrspt $(1,5)$

4924 CALL $W(\operatorname{Pwr}(*), 1,16,16,1, \operatorname{Wrspt}(*), W(*))$

4925 FOR $I=1$ TO 2

$4926 \operatorname{Res}(I, 3)=W(3,4 * I)$

$4927 \operatorname{Res}(I, 4)=W(4,4 * I)$

4928 FOR $\mathrm{J}=5$ TO 7

4929 FOR $K=4 * J-15$ TO $4 * \mathrm{~J}-12$

$4932 \quad \operatorname{Res}(I, J)=\operatorname{Res}(I, J)+W(K, 4 * I) / 4$

4935

4938 NEXT K

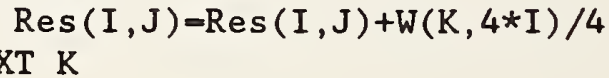

4941 NEXT I

4944 CALL $\operatorname{Tr} 15(\mathrm{~W}(*), \operatorname{Wrspt}(*)$, Opt\$, Stds $(*), \operatorname{Res}(*))$

4947 CALL Stds2(W(*), Wrspt (*), Stds (*), Res (*))

4950 CALL Pratio(W(*), 5, Wrspt(*), Wrspt $(2,12))$

4953 Wrspt $(1,12)=1$

$4956 \quad \operatorname{Res}(1,8)=(\operatorname{Res}(1,3)+\operatorname{Res}(1,4)+4 * \operatorname{Res}(1,5)+4 * \operatorname{Res}(1,6)$ $+4 * \operatorname{Res}(1,7)) / 14$

$4959 \operatorname{Res}(2,8)=(\operatorname{Res}(2,3)+\operatorname{Res}(2,4)+4 * \operatorname{Res}(2,5)+4 * \operatorname{Res}(2,6)$ $+4 * \operatorname{Res}(2,7)) / 14$

4962 MAT Resid-Res

4965 Calib_exit: SUBEND

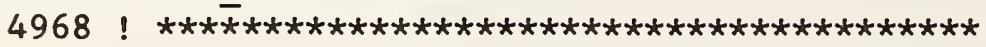

S Calib(

$\begin{array}{lllllll}\text { Calib_exit: } & 4965 & 4902 & 4908 & & & \\ \text { Error_flag } & 4878 & 4893 & 4899 & 4902 & 4905 & 4908 \\ \text { Freq } & 4878 & 4918 & & & & \end{array}$




\begin{tabular}{|c|c|c|c|c|c|c|c|}
\hline $\mathrm{Gl}$ & 4878 & 4896 & & & & & \\
\hline Hi_lo_port & 4878 & 4905 & & & & & \\
\hline I & $\begin{array}{l}4925 \\
4932\end{array}$ & $\begin{array}{l}4926 \\
4941\end{array}$ & 4926 & 4927 & 4927 & 4932 & 4932 \\
\hline $\mathrm{J}$ & 4928 & 4929 & 4929 & 4932 & 4932 & 4938 & \\
\hline K & 4929 & 4932 & 4935 & & & & \\
\hline Opt\$ & 4878 & 4944 & & & & & \\
\hline $\mathbf{P}$ & 4916 & 4918 & 4918 & 4920 & & & \\
\hline Pwr ( & 4878 & 4899 & 4905 & 4924 & & & \\
\hline $\mathrm{Rd}($ & 4878 & 4918 & & & & & \\
\hline $\operatorname{Res}($ & $\begin{array}{l}4884 \\
4944 \\
4956 \\
4962\end{array}$ & $\begin{array}{l}4890 \\
4947 \\
4959\end{array}$ & $\begin{array}{l}4905 \\
4956 \\
4959\end{array}$ & $\begin{array}{l}4926 \\
4956 \\
4959\end{array}$ & $\begin{array}{l}4927 \\
4956 \\
4959\end{array}$ & $\begin{array}{l}4932 \\
4956 \\
4959\end{array}$ & $\begin{array}{l}4932 \\
4956 \\
4959\end{array}$ \\
\hline Resid( & 4878 & 4887 & 4962 & & & & \\
\hline $\mathrm{sp}$ & $\begin{array}{l}4897 \\
4919\end{array}$ & 4899 & 4905 & 4910 & 4917 & 4918 & 4918 \\
\hline Stds ( & 4878 & 4944 & 4947 & & & & \\
\hline v( & 4884 & 4899 & 4905 & & & & \\
\hline W( & $\begin{array}{l}4884 \\
4950\end{array}$ & 4924 & 4926 & 4927 & 4932 & 4944 & 4947 \\
\hline Wrspt ( & $\begin{array}{l}4878 \\
4950\end{array}$ & $\begin{array}{l}4905 \\
4953\end{array}$ & 4918 & 4924 & 4944 & 4947 & 4950 \\
\hline
\end{tabular}


4971 SUB Iparab(P(*),Sp,V(*), Error_flag) !--(81/07/30)-GFE-GEC4974 ON ERROR GOTO Error

4977 DIM $B(1: 16,1: 9), X(1: 9), Y(1: 16)$

4980 MAT $\mathrm{Y}=(-1)$

$4983 \mathrm{~K}=4 * \mathrm{Sp}-4$ ! Adds 4 to column index when $\mathrm{Sp}=2$

$4986 \mathrm{~N}=0$

4989 FOR J=1 TO 16 ! Iterate over rows in "P" matrix

4992 I=J $-\mathrm{N}$

! Index for " $B$ " matrix

$4995 \mathrm{P}=\mathrm{P}(\mathrm{J}, \mathrm{K}+1)$

4998 IF $P>0$ THEN B

! $\mathrm{P}=0$ indicates no data in this row:

$5001 \mathrm{~N}=\mathrm{N}+1$ skip to next one

5004 GOTO Nextj

! \& adjust row index for MAT "B" to lag " $P$ " by one unit

$5007 \mathrm{~B}: \mathrm{B}(\mathrm{I}, 7)=\mathrm{A}=\mathrm{P}(\mathrm{J}, \mathrm{K}+2) / \mathrm{P}$

$5010 \quad \mathrm{~B}(\mathrm{I}, 8)=\mathrm{B}=\mathrm{P}(\mathrm{J}, \mathrm{K}+3) / \mathrm{P}$

$5013 \quad B(I, 9)=C=P(J, K+4) / P$

$5016 \quad B(I, 1)=A * A$

$5019 \quad B(I, 2)=B * B$

$5022 \quad B(I, 3)=C * C$

$5025 \quad B(I, 4)=A * B$

$5028 \quad B(I, 5)=A * C$

$5031 \quad B(I, 6)=B * C$

5034 Nextj: NEXT J

5037 REDIM $B(1: I, 1: 9), Y(1: I)$ ! Drop last $N$ rows in " $B$ " and " $Y$ ",

$5040 \mathrm{CALL} \mathrm{Msoln}(\mathrm{B}(*), \mathrm{Y}(*), \mathrm{X}(*))$ (no data here)

$5043 \mathrm{~B}=(2 * \mathrm{X}(4)-\mathrm{X}(7) * \mathrm{X}(8)) /(2 * \mathrm{X}(1) * \mathrm{X}(8)-\mathrm{X}(4) * \mathrm{X}(7))$

$5046 \mathrm{C}=(2 * \mathrm{X}(5)-\mathrm{X}(7) * \mathrm{X}(9)) /(2 * \mathrm{X}(1) * \mathrm{X}(9)-\mathrm{X}(5) * \mathrm{X}(7))$

$5049 \mathrm{~A}=\mathrm{B}+\mathrm{C}+\mathrm{X}(7) / \mathrm{X}(1)$

$5052 \mathrm{~V}(1)=\mathrm{R} 1=\mathrm{SQR}(\mathrm{X}(2) * \mathrm{~A} * \mathrm{C})$

$5055 \mathrm{~V}(2)=\mathrm{R} 2=\mathrm{SQR}(\mathrm{X}(3) * \mathrm{~A} * \mathrm{~B})$

$5058 \mathrm{~V}(3)=\mathrm{W} 1-\mathrm{SQR}(\mathrm{C})$

$5061 \mathrm{~V}(4)=\mathrm{W} 2=(\mathrm{B}+\mathrm{C}-\mathrm{A}) / 2 / \mathrm{W} 1$

$5064 \mathrm{~V}(5)=\mathrm{W} 3=\mathrm{B}-\mathrm{W} 2 * \mathrm{~W} 2$

5067 SUBEXIT

5070 Error: BEEP

5073 IF ERRN=30 THEN PRINT "Failure in Iparab, Poor set of power data indicated.

$\mathrm{Sp}=$ "; Sp

5076 IF ERRN $>30$ THEN PRINT ERRM\$

5079 Error_flag=10

5082 SUBEND

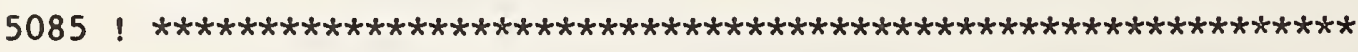

S IparabC

A

$\begin{array}{lllllll}5007 & 5016 & 5016 & 5025 & 5028 & 5049 & 5052\end{array}$

$5055 \quad 5061$

B

$\begin{array}{lllllll}5010 & 5019 & 5019 & 5025 & 5031 & 5043 & 5049\end{array}$

B(

$5055 \quad 5061 \quad 5064$

$\begin{array}{lllllll}4977 & 5007 & 5010 & 5013 & 5016 & 5019 & 5022\end{array}$ 


\begin{tabular}{|c|c|c|c|c|c|c|c|}
\hline & 5025 & 5028 & 5031 & 5037 & 5040 & & \\
\hline B : & 5007 & 4998 & & & & & \\
\hline C & $\begin{array}{l}5013 \\
5052\end{array}$ & $\begin{array}{l}5022 \\
5058\end{array}$ & $\begin{array}{l}5022 \\
5061\end{array}$ & 5028 & 5031 & 5046 & 5049 \\
\hline Error: & 5070 & 4974 & & & & & \\
\hline Error_flag & 4971 & 5079 & & & & & \\
\hline Fparab: & 5088 & & & & & & \\
\hline I & $\begin{array}{l}4992 \\
5025 \\
4995 \\
5007\end{array}$ & $\begin{array}{l}5007 \\
5028 \\
5007 \\
5010\end{array}$ & $\begin{array}{l}5010 \\
5031 \\
5010 \\
5013\end{array}$ & $\begin{array}{l}5013 \\
5037 \\
5013\end{array}$ & $\begin{array}{l}5016 \\
5037 \\
5034\end{array}$ & $\begin{array}{l}5019 \\
4989 \\
4983\end{array}$ & $\begin{array}{l}5022 \\
4992 \\
4995\end{array}$ \\
\hline $\mathrm{N}$ & 4986 & 4992 & 5001 & 5001 & & & \\
\hline Nextj: & 5034 & 5004 & & & & & \\
\hline$P$ & 4995 & 4998 & 5007 & 5010 & 5013 & & \\
\hline$P($ & 4971 & 4995 & 5007 & 5010 & 5013 & & \\
\hline R1 & 5052 & & & & & & \\
\hline R2 & 5055 & & & & & & \\
\hline $\mathrm{Sp}$ & 4971 & 4983 & 5073 & & & & \\
\hline V( & 4971 & 5052 & 5055 & 5058 & 5061 & 5064 & \\
\hline W1 & 5058 & 5061 & & & & & \\
\hline W2 & 5061 & 5064 & 5064 & & & & \\
\hline W3 & 5064 & & & & & & \\
\hline $\mathrm{XC}$ & $\begin{array}{l}4977 \\
5043 \\
5046\end{array}$ & $\begin{array}{l}5040 \\
5043 \\
5046\end{array}$ & $\begin{array}{l}5043 \\
5046 \\
5049\end{array}$ & $\begin{array}{l}5043 \\
5046 \\
5049\end{array}$ & $\begin{array}{l}5043 \\
5046 \\
5052\end{array}$ & $\begin{array}{l}5043 \\
5046 \\
5055\end{array}$ & $\begin{array}{l}5043 \\
5046\end{array}$ \\
\hline $\mathrm{YC}$ & 4977 & 4980 & 5037 & 5040 & & & \\
\hline
\end{tabular}


5088 Fparab: SUB Fparab $(\mathrm{P}(*), \mathrm{Sp}, \mathrm{V}(*), \mathrm{W}(*), \operatorname{Res}(*)$, Error_flag,Hi_10 port)

! -. (81/07/30) -......-.GFE-GEC - ....-

5091 ! $\mathrm{V}(1) \ldots \mathrm{V}(5)=\mathrm{R} 1, \mathrm{R} 2, \mathrm{~W} 1$, W2, W3

5094 DIM $B(1: 16,1: 5), X(1: 5), Y(1: 16)$

$5097 \mathrm{~K}=4 * \mathrm{Sp}-4$ ! Adds 4 to column index when $\mathrm{Sp}=2$

$5100 \mathrm{Ab}=(\mathrm{V}(4) * \mathrm{~V}(4)+\mathrm{V}(5)) *\left((\mathrm{~V}(4)-\mathrm{V}(3))^{\wedge} 2+\mathrm{V}(5)\right)$ ! Normalize by ab

5103 FOR $\mathrm{H}=1$ TO 10 ! 10 = upper limit to number of iterations

$5106 \mathrm{~N}: \mathrm{N}=0$

5109 FOR J=1 TO 16 ! Iterates over rows in "P" matrix

$5112 \mathrm{I}=\mathrm{J}-\mathrm{N}$

! Index for "B" matrix

$5115 \mathrm{P}=\mathrm{P}(\mathrm{J}, \mathrm{K}+1)$

5118 IF $\mathrm{P}>0$ THEN $\mathrm{P} \quad \mathrm{!} \mathrm{P}=0$ indicates no data in this row: skip to next one

$5121 \mathrm{~N}=\mathrm{N}+1$

! \& adjust row index in MAT "B" to lag "P" by one unit

5124 GOTO Nextj

$5127 \mathrm{P}: \mathrm{P} 3=\mathrm{P}(\mathrm{J}, \mathrm{K}+2) / \mathrm{P}$

$5130 \quad \mathrm{P} 5=\mathrm{P}(\mathrm{J}, \mathrm{K}+3) / \mathrm{P}$

$5133 \quad \mathrm{P} 6=\mathrm{P}(\mathrm{J}, \mathrm{K}+4) / \mathrm{P}$

$5136 \quad A=(V(1) * P 5-P 3) / V(3)-V(3)$

$5139 \quad B=P 3+V(4) *(V(4)+A)+V(5)-V(2) * P 6$

$5142 \quad C=A * A-4 * P 3$

$5145 \quad P=2 *(B * V(4)+A * V(5))$

$5148 \quad \mathrm{Y}(\mathrm{I})=-\mathrm{B} * \mathrm{~B}-\mathrm{C} * \mathrm{~V}(5)$

5151 ! Parab_res $\left(\mathrm{SP}_{\mathrm{P}} \mathrm{J}\right)=\mathrm{Y}(\mathrm{I}) / \mathrm{Ab}$ ! Place normalized residual in

$5154 \quad B(I, 1)=P * P 5 / V(3)$ approprate column

$5157 \quad B(I, 2)=-2 * B * P 6$

$5160 \quad B(I, 3)=P *(-A / V(3)-2)$

$5163 \quad B(I, 4)=2 * B *(2 * V(4)+A)$

$5166 \quad B(I, 5)=2 * B+C$

5169 Nextj: NEXT J

5172 REDIM $B(1: I, 1: 5), Y(1: I)$ ! Drop bottom $N$ rows (no data here)

5175 Q=DOT $(Y, Y)$

5178 IF $\mathrm{H}>1$ THEN Qa

5181 Qi=Q

5184 GOTO T

5187 Qa: IF Q<T*1.001 THEN Qb

Save initial residual

! Initialize $\mathrm{T}$

$5190 \mathrm{~L}-2 * \mathrm{~L}$

5193 IF L $>70$ THEN Error_flag=2

5196 IF L $>70$ THEN Error

5199 MAT $\mathrm{X}=\mathrm{X} /(\mathrm{L})$

5202 MAT $V=V-X$

5205 GOTO N

$5208 \mathrm{Qb}$ : IF (Q>T*.99) OR (Q<1E-16) THEN Res

$5211 \mathrm{~T}: \mathrm{T}=\mathrm{Q}$

$5214 \mathrm{~L}=1$

5217 CALL Msoln(B(*), Y(*), X(*))

5220 MAT $\mathrm{V}=\mathrm{V}+\mathrm{X}$

5223 NEXT H

5226 PRINT "Ten iterations performed by Fparab"

5229 Error_flag=1

5232 Error: BEEP 
5235 PRINT "Abnormal exit from Fparab routine. Poor initial estimate implied"

5238 Res: $A b=(V(4) * V(4)+V(5)) *\left((V(4)-V(3))^{\wedge} 2+V(5)\right)$

\section{1}

$\operatorname{Res}(\mathrm{Sp}, 1)=\mathrm{SQR}(\mathrm{Q} /(\mathrm{I}-5)) / \mathrm{Ab}$

! Normalize by $a b$

\section{4}

$\operatorname{Res}(\mathrm{Sp}, 2)-\mathrm{SQR}(\mathrm{Q} \mathrm{i} / \mathrm{Q})$

5247 IF $V(5)>0$ THEN Ex

5250 PRINT "Error in Fparab, $(\operatorname{Im}(W 2))^{\wedge} 2=" ; V(5)$

5253 V5-SQR (ABS (V(5)))

5256 Error_flag-Error_flag+2

5259 GOTO Err

5262 Ex: $V(5)=S Q R(V(5))$ ! A plus or minus sign may be required depending upon

5265 IF NOT Hi_lo_port THEN $V(5)=-V(5)$

5268 IF $\operatorname{ABS}(\mathrm{V}(\overline{5}) / \bar{N}(4))>.05$ THEN Exit

5271 BEEP

how detector "numbers" are assigned

5274 PRINT "Poor initial parabolid estimate indicated"

5277 PRINT

5280 Err: BEEP

5283 PRINT "Six-port="; Sp

5286 PRINT "Zeta= ";V(1)

5289 PRINT "Rho= ";V(2)

5292 PRINT "Wl= ";V(3)

5295 PRINT $" \operatorname{Re}(W 2)=\quad " ; V(4)$

5298 PRINT " $\operatorname{Im}(W 2)=\quad$ ";V(5)

5301 PAUSE

5304 Exit: FOR $I=1$ TO 5

$5307 \mathrm{~W}(\mathrm{Sp}, \mathrm{I})-\mathrm{V}(\mathrm{I})$

5310 NEXT I

5313 SUBEND

5316 ! ***************************

$S$ Fparab(

$\begin{array}{llllllll}\text { A } & 5136 & 5139 & 5142 & 5142 & 5145 & 5160 & 5163 \\ \mathrm{Ab} & 5100 & 5238 & 5241 & & & & \\ \mathrm{~B} & 5139 & 5145 & 5148 & 5148 & 5157 & 5163 & 5166 \\ \mathrm{~B}( & 5094 & 5154 & 5157 & 5160 & 5163 & 5166 & 5172 \\ & 5217 & & & & & & \\ \text { C } & 5142 & 5148 & 5166 & & & & \\ \text { Err: } & 5280 & 5259 & & & & & \\ \text { Error: } & 5232 & 5196 & & & & & \\ \text { Error_flag } & 5088 & 5193 & 5229 & 5256 & 5256 & & \\ \text { Ex: } & 5262 & 5247 & & & & & \\ \text { Exit: } & 5304 & 5268 & & & & & \end{array}$


H

H1_10_port

I

$\mathrm{J}$

$\mathbf{K}$

L

$\mathrm{N}$

$\mathrm{N}:$

Nextj :

$\mathbf{P}$

P (

P3

P5

P6

P:

Q

Qa:

Qb :

Qi

$\operatorname{Res}($

Res :

$\mathrm{Sp}$

T

T:

V(
$5103 \quad 5178 \quad 5223$

$5088 \quad 5265$

$\begin{array}{lllllll}5112 & 5148 & 5154 & 5157 & 5160 & 5163 & 5166\end{array}$

$\begin{array}{lllllll}5172 & 5172 & 5241 & 5304 & 5307 & 5307 & 5310\end{array}$

$\begin{array}{lllllll}5109 & 5112 & 5115 & 5127 & 5130 & 5133 & 5169\end{array}$

$\begin{array}{lllll}5097 & 5115 & 5127 & 5130 & 5133\end{array}$

$\begin{array}{llllll}5190 & 5190 & 5193 & 5196 & 5199 & 5214\end{array}$

$\begin{array}{llll}5106 & 5112 & 5121 & 5121\end{array}$

$5106 \quad 5205$

$5169 \quad 5124$

$\begin{array}{lllllll}5115 & 5118 & 5127 & 5130 & 5133 & 5145 & 5154\end{array}$

5160

$\begin{array}{lllll}5088 & 5115 & 5127 & 5130 & 5133\end{array}$

$5127 \quad 5136 \quad 5139 \quad 5142$

$5130 \quad 5136 \quad 5154$

$5133 \quad 5139 \quad 5157$

$5127 \quad 5118$

$\begin{array}{lllllll}5175 & 5181 & 5187 & 5208 & 5208 & 5211 & 5241\end{array}$ 5244

$5187 \quad 5178$

$5208 \quad 5187$

$5181 \quad 5244$

$5088 \quad 5241 \quad 5244$

$5238 \quad 5208$

$\begin{array}{llllll}5088 & 5097 & 5241 & 5244 & 5283 & 5307\end{array}$

$5187 \quad 5208 \quad 5211$

$5211 \quad 5184$

$\begin{array}{lllllll}5088 & 5100 & 5100 & 5100 & 5100 & 5100 & 5100\end{array}$

$\begin{array}{lllllll}5136 & 5136 & 5136 & 5139 & 5139 & 5139 & 5139\end{array}$

$\begin{array}{lllllll}5145 & 5145 & 5148 & 5154 & 5160 & 5163 & 5202\end{array}$ 
$\begin{array}{lllllll}5202 & 5220 & 5220 & 5238 & 5238 & 5238 & 5238\end{array}$

$\begin{array}{lllllll}5238 & 5238 & 5247 & 5250 & 5253 & 5262 & 5262\end{array}$

$\begin{array}{lllllll}5265 & 5265 & 5268 & 5268 & 5286 & 5289 & 5292\end{array}$

$5295 \quad 5298 \quad 5307$

V5 5253

W( $\quad 5088 \quad 5307$

$\begin{array}{lllllll} & 5094 & 5199 & 5199 & 5202 & 5217 & 5220\end{array}$

Y 5175

$\begin{array}{llllll}Y & 5094 & 5148 & 5172 & 5175 & 5217\end{array}$ 
5319 SUB Pratio(W(*), $\operatorname{Row}, \operatorname{Wr}(*), R) \quad !-(81 / 05 / 06)-$ GFE - -

5322 DIM A(1:8)

5325 FOR $I=1$ TO 4

$5328 \mathrm{~J}=$ Row $+\mathrm{I}-1$

5331 CALL Cmpy(Wr( $(1,10), \operatorname{Wr}(1,11), W(J, 1), W(J, 2), A x, A y)$

5334 CALL Csub(Wr$(1,6), \operatorname{Wr}(1,7), A x, A y, A x, A y)$

5337 CALL Csub $(W(J, 5), W(J, 6), W r(2,8), W r(2,9), B x, B y)$

5340 CALL Csub $(W(J, 1), W(J, 2), \operatorname{Wr}(1,8), W r(1,9), C x, C y)$

5343 CALL Cmpy $(W r(2,10), \operatorname{Wr}(2,11), W(J, 5), W(J, 6), D x, D y)$

5346 CALL Csub $(\operatorname{Wr}(2,6), \operatorname{Wr}(2,7), \mathrm{Dx}, \mathrm{Dy}, \mathrm{Dx}, \mathrm{Dy})$

$5349 \mathrm{~A}(2 * \mathrm{I}-1)=(\mathrm{Ax} * \mathrm{Ax}+\mathrm{Ay} * \mathrm{Ay}) /(\mathrm{Bx} * \mathrm{Bx}+\mathrm{By} * \mathrm{By}) * \mathrm{~W}(\mathrm{~J}, 3) / \mathrm{W}(\mathrm{J}, 7)$

$5352 A(2 * I)=(C x * C x+C y * C y) /(D x * D x+D y * D y) * W(J, 3) / W(J, 7)$

5355 NEXT I

$5358 \mathrm{R}=\operatorname{SUM}(\mathrm{A}) / 8$

5361 MAT $A=A-(R)$

$5364 \operatorname{Res}=\operatorname{SQR}(\operatorname{DOT}(\mathrm{A}, \mathrm{A}) / 7) / \mathrm{R}$ ! (Std dev)/(mean)

5367 SUBEND

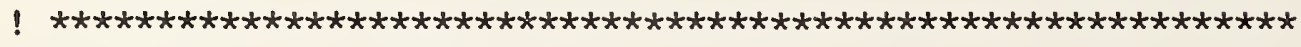

S Pratio(

A

5364

Al

5322

$\mathrm{Ax}$

$\begin{array}{lllll}5331 & 5334 & 5334 & 5349 & 5349\end{array}$

Ay

$\begin{array}{lllll}5331 & 5334 & 5334 & 5349 & 5349\end{array}$

$\mathrm{Bx}$

$5337 \quad 5349 \quad 5349$

By

$5337 \quad 5349 \quad 5349$

$C x$

Cy

$\begin{array}{lll}5340 & 5352 & 5352\end{array}$

$\mathrm{Dx}$

$5340 \quad 5352 \quad 5352$

$\begin{array}{lllll}5343 & 5346 & 5346 & 5352 & 5352\end{array}$

Dy

$\begin{array}{lllll}5343 & 5346 & 5346 & 5352 & 5352\end{array}$

I

$\begin{array}{lllll}5325 & 5328 & 5349 & 5352 & 5355\end{array}$

$\mathrm{J}$

$\begin{array}{lllllll}5328 & 5331 & 5331 & 5337 & 5337 & 5340 & 5340\end{array}$

$\mathbf{R}$

$\begin{array}{llllll}5343 & 5343 & 5349 & 5349 & 5352 & 5352\end{array}$

$\begin{array}{llll}5319 & 5358 & 5361 & 5364\end{array}$

Res

5364

Row

$5319 \quad 5328$

W(

$5319 \quad 5331$

5343

5331

5337

5337

5340

5340

$\operatorname{Wr}($

5319

5331

5349

5349

$5352 \quad 5352$

5340

5340

$\begin{array}{lll}5334 & 5334 & 5337\end{array}$

5337 
5373 SUB Stds2(W(*), $\operatorname{Wr}(*), S(*), \operatorname{Res}(*)) !-(81 / 12 / 18)-G F E-. . .$.

5376 ! Modification of "Stds" for use with Type $N$ connector \& "Tr13"

5379 DIM $G(1: 4,1: 4), T(1: 6)$

$5382 \operatorname{CALL} \operatorname{Wg}(\operatorname{Wr}(*), 1,1, W(3,1), W(3,2), S(4,1), S(4,2))$

5385 CALL $\operatorname{Wg}(\operatorname{Wr}(*), 2,1, W(3,5), W(3,6), S(5,1), S(5,2))$

5388 CALL $\operatorname{Wg}(\operatorname{Wr}(*), 1,1, W(4,1), W(4,2), \mathrm{S}(6,1), \mathrm{S}(6,2))$

5391 CALL $\operatorname{Wg}(\operatorname{Wr}(*), 2,1, W(4,5), W(4,6), S(7,1), S(7,2))$

5394 FOR I=1 TO 4

5397 FOR $\mathrm{Sp}=1$ TO 2

$5400 \mathrm{~J}-(\mathrm{Sp}=1)+3 *(\mathrm{Sp}=2)$

$5403 \mathrm{~K}-\mathrm{J}+2 *(\mathrm{Sp}=2)$

$5406 \operatorname{CALL} W g(W r(*), S p, 0, W(I+12, K), W(I+12, K+1), G(I, J), G(I, J+1))$

5409 NEXT SP

5412 NEXT I

5415 CALL $\operatorname{Rtpt}(G(*), 1, T(*), \operatorname{Res}(1,11))$

5418 CALL Polar(T(2), T(5), S $(8,1), S(8,2)$ )

5421 CALL Cmpy(T(2), T(5), T(3), T(6), X,Y)

5424 CALL Csub(T(1), T(4), X, Y,X,Y)

5427 CALL Polar (X, Y,R, T)

$5430 \mathrm{~S}(9,1)=\mathrm{SQR}(\mathrm{R})$

$5433 \mathrm{~S}(9,2)=\mathrm{T} / 2-180 *(\mathrm{~T}>0)$ ! Default value between $-180 \& 0$

5436 CALL Polar $(-\mathrm{T}(3),-\mathrm{T}(6), \mathrm{S}(10,1), \mathrm{S}(10,2))$

5439 SUBEND

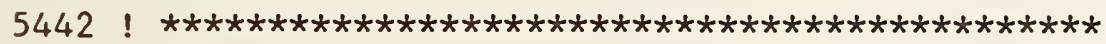

S Stds 2 (

G(

I

$\mathrm{J}$

$\mathrm{K}$

R

$\operatorname{Res}($

S

$\mathrm{Sp}$

$\mathrm{T}$

T

$\operatorname{Wr}($

$\mathrm{X}$

Y

$5379 \quad 5406 \quad 5406 \quad 5415$

$\begin{array}{llllll}5394 & 5406 & 5406 & 5406 & 5406 & 5412\end{array}$

$5400 \quad 5403 \quad 5406 \quad 5406$

$5403 \quad 5406 \quad 5406$

$5427 \quad 5430$

$5373 \quad 5415$

$\begin{array}{lllllll}5373 & 5382 & 5382 & 5385 & 5385 & 5388 & 5388\end{array}$

$\begin{array}{lllllll}5391 & 5391 & 5418 & 5418 & 5430 & 5433 & 5436\end{array}$

5436

$\begin{array}{llllll}5397 & 5400 & 5400 & 5403 & 5406 & 5409\end{array}$

$5427 \quad 5433 \quad 5433$

$\begin{array}{lllllll}5379 & 5415 & 5418 & 5418 & 5421 & 5421 & 5421 \\ 5421 & 5424 & 5424 & 5436 & 5436 & & \end{array}$

$\begin{array}{llllll}5373 & 5382 & 5385 & 5388 & 5391 & 5406\end{array}$

$\begin{array}{llll}5421 & 5424 & 5424 & 5427\end{array}$

$\begin{array}{llll}5421 & 5424 & 5424 & 5427\end{array}$ 
5445 SUB $\operatorname{Tr} 15(W(*), W r(*)$, Opt $\$, \operatorname{Stds}(*), \operatorname{Res}(*)) !-(82 / 04 / 14)-$ GFE - 5448 ! Modification of "Trl" routine. Gives three options

5451 ! Opt $\$=1$

5454 ! Opt $\$=2 \mathrm{~L},(2 \mathrm{R})$

$5457 !$

5463 ! Option=3

$5466 !$
Standard TRL routine

For Type $\mathrm{N}$ connector, assumes equal mag gamma for "opens"

\& assigns phase of 180 to termination or "short" on either Six=port\#1 (left), or if Opt $\$=2 R$ to Six-port\#2 (right)

Assumes equal mag gamma on "opens", equal phase on "shorts"

Obtains Mag Gamma from opens, phase from shorts

5469 IF (Opt $\$=" 1 ")$ OR (Opt $\$=" 2 L ")$ OR (Opt $\$=" 2 R ")$ OR (Opt $\$=3 ")$ THEN 5478

5472 PRINT "IMPROPER FORMAT FOR Option\$"

5475 GOTO 5703

5478 DIM S $(1: 6)$

5481 DEG

5484 CALL Rtpt(W(*), 5, S(*), Res $(1,9))$

5487 CALL Ident(Tax, S(1), Tbx, S(2), Tcx, S(3), Tay, S(4), Tby, S(5), Tcy, $S(6))$ ! "Thru"5490 CALL Rtpt(W(*),9,S(*), $\operatorname{Res}(1,10))$

5493 CALL Ident(Lax, S (1), Lbx, S (2), Lcx, S (3), Lay, S (4), Lby, S (5), Lcy, $s(6))$ ! "Line"

5496 CALL Cmpy (Lbx, Lby, Tcx, Tcy, Ax, Ay)

5499 CALL Csub (Lax, Lay, Ax, Ay, Max, May)

5502 CALL Cmpy(Tax, Tay, Lbx, Lby,Ax, Ay)

5505 CALL Cmpy (Lax, Lay, Tbx, Tby, Bx, By)

5508 CALL Csub (Ax, Ay, Bx, By, Mbx, Mby)

5511 CALL Csub (Lcx, Lcy, Tcx, Tcy, Mcx, Mcy)

5514 CALL Cmpy (Tbx, Tby, Lcx, Lcy, Ax, Ay)

5517 CALL Csub (Tax, Tay, Ax, Ay, Mdx, Mdy)

5520 CALL Csub (Mdx/2, Mdy/2, Max/2, May/2, Bx, By)

5523 CALL Cdiv(Mcx, Mcy,Bx, By, Ax, Ay)

5520 CALL Cdiv (-Mbx, -Mby, Bx, By, Cx, Cy)

5529 CALl Cmpy (Ax, Ay, Cx, Cy, Dx, Dy)

5532 CALL Csub (1, 0, Dx, Dy,Dx, Dy)

$5535 R=S Q R(S Q R(D x * D x+D y * D y))$

$5538 \mathrm{~T}=(\operatorname{ATN}(\mathrm{Dy} /(\mathrm{Dx}+1 \mathrm{E}-20))+180 *(\mathrm{Dx}<0) * \mathrm{SGN}(\mathrm{Dy}+1 \mathrm{E}-20)) / 2$ ! Root choice made here

5541 CALL Cadd $(1,0, R * \operatorname{COS}(T), R * \operatorname{SIN}(T), D x, D y)$

5544 CALL Cdiv (-Cx, - Cy, Dx, Dy, Abx, Aby)

5547 CALL Cdiv (-Ax, -Ay, Dx, Dy, Acx, Acy)

5550 CALL Cmpy (Mbx, Mby, Acx, Acy, Ax, Ay)

5553 CALL Cadd (Ax, Ay, Max, May, Ax, Ay)

! Compute Exp(-gamma1)

for line

5556 CALL Cmpy (Mcx, Mcy, Abx, Aby, Bx, By)

5559 CALL Cadd (Bx, By, Mdx, Mdy, Bx, By)

5562 CALL $\operatorname{Cdiv}(A x, A y, B x, B y, X, Y)$

5565 CALI Polar $(X, Y, R, T)$

$5568 \operatorname{Stds}(3,1)=\mathrm{SQR}(\mathrm{R})$

$5571 \operatorname{Stds}(3,2)=\mathrm{T} / 2-180 *(\mathrm{~T}>0) \quad$ ! Default value of 1 ine length between $-180 \& 0$

5574 CALL Cmpy (Acx, Acy, Tbx, Tby, Dx, Dy)

5577 CALL Csub (1, 0, Dx, Dy, Dx, Dy) 
5580 CALl Cmpy(Acx, Acy, Tax, Tay, Ax, Ay)

5583 CALL Csub (Tcx, Tcy, Ax, Ay, Ax, Ay)

5586 CALL Cdiv(Ax, Ay, Dx, Dy, Bcx, Bcy)

5589 CALl Cmpy (Abx, Aby, Tcx, Tcy, Ax, Ay)

5592 CALl Csub(Tax, Tay, Ax, Ay, Ax, Ay)

5595 CALl Cdiv(Ax, Ay, Dx, Dy, Px, Py)

5598 CALl Csub (Tbx, Tby, Abx, Aby, Bx, By)

5601 CALL Cdiv(Bx, By, Ax, Ay, Bbx, Bby)

5604 FOR K-3 TO 4

5607 IF (Opt $\$=" 2 R ")$ AND $(K=3)$ THEN 5628

5610 CALL Cmpy (Acx, Acy, $W(K, 1), W(K, 2), A x, A y)$

5613 CALL $\operatorname{Csub}(1,0, A x, A y, A x, A y)$

5616 CALL Csub ( $W(K, 1), W(K, 2), A b x, A b y, B x, B y)$

5619 CALL Cdiv(Bx, By, Ax, Ay, Ax, Ay)

5622 IF $\mathrm{K}=3$ THEN CALL Polar (-Ax, - Ay, R, T)

5625 IF (Opt $\left.\$=" 2 L^{\prime \prime}\right)$ AND $(\mathrm{K}=3)$ THEN 5679

5628 CALL Cadd $(W(K, 5), W(K, 6), B c x, B c y, B x, B y)$

5631 CALL Cmpy $(W(K, 5), W(K, 6), B b x, B b y, C x, C y)$

5634 CALL Cadd $(1,0, C x, C y, C x, C y)$

5637 CALL Cdiv (Cx, Cy, Bx, By, Bx, By)

5640 IF (Opt\$ $\bigcirc$ "2R") OR (K=4) THEN 5652

5643 CALL Cmpy (Px, Py, Bx, By, Cx, Cy)

5646 CALL Polar (-Cx, - Cy, R, T)

5649 GOTO 5679

5652 CALL Cmpy (Ax, Ay, Bx, By, Qx, Qy)

5655 CALL Cmpy (Px, Py, Qx, Qy, Ax, Ay)

5658 IF $\mathrm{K}=4$ THEN 5682

$5661 \mathrm{H}=\mathrm{T}$

$5664 \mathrm{~T}=(\operatorname{ATN}(\mathrm{Ay} /(\mathrm{Ax}+1 \mathrm{E}-20))+180 *(\mathrm{Ax}<0) * \mathrm{SGN}(\mathrm{Ay}+1 \mathrm{E}-20)) / 2$

5667 Q-T-H ! This assumes calibration with nominal short

5670 IF $A B S(Q)>180$ THEN $Q=Q-360 * S G N(Q)$

5673 IF $\mathrm{ABS}(\mathrm{Q})>90$ THEN $\mathrm{T}=\mathrm{T}+180$

5676 IF Opt $\$=" 1$ " THEN 5682

5679 NEXT $K$

$5682 \mathrm{R}=\mathrm{SQR}(\mathrm{SQR}(\mathrm{Ax} * \mathrm{Ax}+\mathrm{Ay} * \mathrm{Ay}))$

5685 CALL Rect( $R, T$, Aax, Aay)

5688 CALL Cdiv(Px, Py, Aax, Aay, Bax, Bay)

5691 CALl Cmpy (Acx, Acy, Aax, Aay, Acx, Acy)

5694 CALl Cmpy (Bbx, Bby, Bax, Bay,Bbx,Bby)

5697 CALL Ident $(\operatorname{Wr}(1,6), \operatorname{Aax}, \operatorname{Wr}(1,7), \operatorname{Aay}, \operatorname{Wr}(1,8), A b x, \operatorname{Wr}(1,9), A b y$, $\operatorname{Wr}(1,10), \operatorname{Acx}, \operatorname{Wr}(1,11), \operatorname{Acy})$

5700 CALL Ident $(\operatorname{Wr}(2,6), \operatorname{Bax}, \operatorname{Wr}(2,7), \operatorname{Bay}, \operatorname{Wr}(2,8),-\operatorname{Bcx}, \operatorname{Wr}(2,9),-\operatorname{Bcy}$,

5703 SUBEND

$$
\operatorname{Wr}(2,10),-\operatorname{Bbx}, \operatorname{Wr}(2,11),-\operatorname{Bby})
$$

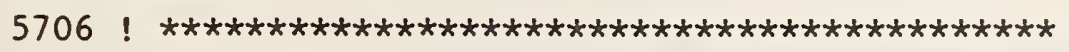

$\mathrm{S} \operatorname{Tr} 15($

Aax

$5685 \quad 5688 \quad 5691 \quad 5697$

Aay

$\begin{array}{llll}5685 & 5688 \quad 5691 \quad 5697\end{array}$

$\mathrm{Abx}$

$\begin{array}{llllll}5544 & 5556 & 5589 & 5598 & 5616 & 5697\end{array}$ 


\begin{tabular}{|c|c|c|c|c|c|c|c|}
\hline Aby & 5544 & 5556 & 5589 & 5598 & 5616 & 5697 & \\
\hline$A c x$ & $\begin{array}{l}5547 \\
5697\end{array}$ & 5550 & 5574 & 5580 & 5610 & 5691 & 5691 \\
\hline acy & $\begin{array}{l}5547 \\
5697\end{array}$ & 5550 & 5574 & 5580 & 5610 & 5691 & 5691 \\
\hline$A x$ & $\begin{array}{l}5496 \\
5529 \\
5583 \\
5601 \\
5652\end{array}$ & $\begin{array}{l}5499 \\
5547 \\
5583 \\
5610 \\
5655\end{array}$ & $\begin{array}{l}5502 \\
5550 \\
5586 \\
5613 \\
5664\end{array}$ & $\begin{array}{l}5508 \\
5553 \\
5589 \\
5613 \\
5664\end{array}$ & $\begin{array}{l}5514 \\
5553 \\
5592 \\
5619 \\
5682\end{array}$ & $\begin{array}{l}5517 \\
5562 \\
5592 \\
5619 \\
5682\end{array}$ & $\begin{array}{l}5523 \\
5580 \\
5595 \\
5622\end{array}$ \\
\hline Ay & $\begin{array}{l}5496 \\
5529 \\
5583 \\
5601 \\
5652\end{array}$ & $\begin{array}{l}5499 \\
5547 \\
5583 \\
5610 \\
5655\end{array}$ & $\begin{array}{l}5502 \\
5550 \\
5586 \\
5613 \\
5664\end{array}$ & $\begin{array}{l}5508 \\
5553 \\
5589 \\
5613 \\
5664\end{array}$ & $\begin{array}{l}5514 \\
5553 \\
5592 \\
5619 \\
5682\end{array}$ & $\begin{array}{l}5517 \\
5562 \\
5592 \\
5619 \\
5682\end{array}$ & $\begin{array}{l}5523 \\
5580 \\
5595 \\
5622\end{array}$ \\
\hline Bax & 5688 & 5694 & 5700 & & & & \\
\hline Bay & 5688 & 5694 & 5700 & & & & \\
\hline $\mathrm{Bbx}$ & 5601 & 5631 & 5694 & 5694 & 5700 & & \\
\hline Bby & 5601 & 5631 & 5694 & 5694 & 5700 & & \\
\hline $\mathrm{Bcx}$ & 5586 & 5628 & 5700 & & & & \\
\hline Bcy & 5586 & 5628 & 5700 & & & & \\
\hline $\mathrm{Bx}$ & $\begin{array}{l}5505 \\
5559 \\
5637 \\
5523 \\
5601 \\
5652\end{array}$ & $\begin{array}{l}5508 \\
5562 \\
5637 \\
5526 \\
5616\end{array}$ & $\begin{array}{l}5520 \\
5598 \\
5643 \\
5556 \\
5619\end{array}$ & $\begin{array}{l}5523 \\
5601 \\
5652 \\
5559 \\
5628\end{array}$ & $\begin{array}{l}5526 \\
5616 \\
5505 \\
5559 \\
5637\end{array}$ & $\begin{array}{l}5556 \\
5619 \\
5508 \\
5562 \\
5637\end{array}$ & $\begin{array}{l}5559 \\
5628 \\
5520 \\
5598 \\
5643\end{array}$ \\
\hline$C x$ & $\begin{array}{l}5526 \\
5643\end{array}$ & $\begin{array}{l}5529 \\
5646\end{array}$ & 5544 & 5631 & 5634 & 5634 & 5637 \\
\hline Cy & $\begin{array}{l}5526 \\
5643\end{array}$ & $\begin{array}{l}5529 \\
5646\end{array}$ & 5544 & 5631 & 5634 & 5634 & 5637 \\
\hline $\mathrm{Dx}$ & $\begin{array}{l}5529 \\
5541 \\
5595\end{array}$ & $\begin{array}{l}5532 \\
5544\end{array}$ & $\begin{array}{l}5532 \\
5547\end{array}$ & $\begin{array}{l}5535 \\
5574\end{array}$ & $\begin{array}{l}5535 \\
5577\end{array}$ & $\begin{array}{l}5538 \\
5577\end{array}$ & $\begin{array}{l}5538 \\
5586\end{array}$ \\
\hline Dy & $\begin{array}{l}5529 \\
5541 \\
5595\end{array}$ & $\begin{array}{l}5532 \\
5544\end{array}$ & $\begin{array}{l}5532 \\
5547\end{array}$ & $\begin{array}{l}5535 \\
5574\end{array}$ & $\begin{array}{l}5535 \\
5577\end{array}$ & $\begin{array}{l}5538 \\
5577\end{array}$ & $\begin{array}{l}5538 \\
5586\end{array}$ \\
\hline $\mathrm{H}$ & 5661 & 5667 & & & & & \\
\hline
\end{tabular}


$\operatorname{Lax}$

Lay

Lbx

Lby

Lcx

Lcy

$\operatorname{Max}$

May

Mbx

Mby

Mcx

Mcy

$\operatorname{Mdx}$

Mdy

Opt\$

$\mathrm{Px}$

Py

Q

Qx

Qy

R

$\operatorname{Res}($

S(

$5493 \quad 5499 \quad 5505$

$5493 \quad 5499 \quad 5505$

$5493 \quad 5496 \quad 5502$

$5493 \quad 5496 \quad 5502$

$5493 \quad 5511 \quad 5514$

$5493 \quad 5511 \quad 5514$

$5499 \quad 5520 \quad 5553$

$5499 \quad 5520 \quad 5553$

$5508 \quad 5526 \quad 5550$

$5508 \quad 5526 \quad 5550$

$5511 \quad 5523 \quad 5556$

$5511 \quad 5523 \quad 5556$

$5517 \quad 5520 \quad 5559$

$5517 \quad 5520 \quad 5559$

$\begin{array}{lllllll}5445 & 5469 & 5469 & 5469 & 5469 & 5607 & 5625\end{array}$

$5640 \quad 5676$

$\begin{array}{llll}5595 & 5643 & 5655 & 5688\end{array}$

$\begin{array}{llll}5595 & 5643 & 5655 & 5688\end{array}$

$\begin{array}{llllll}5667 & 5670 & 5670 & 5670 & 5670 & 5673\end{array}$

$5652 \quad 5655$

$5652 \quad 5655$

$\begin{array}{lllllll}5535 & 5541 & 5541 & 5565 & 5568 & 5622 & 5646\end{array}$

$5682 \quad 5685$

$5445 \quad 5484 \quad 5490$

$\begin{array}{lllllll}5478 & 5484 & 5487 & 5487 & 5487 & 5487 & 5487\end{array}$

$\begin{array}{lllllll}5487 & 5490 & 5493 & 5493 & 5493 & 5493 & 5493\end{array}$

5493 


$\begin{array}{llllllll}\text { Stds } & 5445 & 5568 & 5571 & & & & \\ \text { T } & 5538 & 5541 & 5541 & 5565 & 5571 & 5571 & 5622 \\ & 5646 & 5661 & 5664 & 5667 & 5673 & 5673 & 5685 \\ \text { Tax } & 5487 & 5502 & 5517 & 5580 & 5592 & & \\ \text { Tay } & 5487 & 5502 & 5517 & 5580 & 5592 & & \\ \text { Tbx } & 5487 & 5505 & 5514 & 5574 & 5598 & & \\ \text { Tby } & 5487 & 5505 & 5514 & 5574 & 5598 & & \\ \text { Tcx } & 5487 & 5496 & 5511 & 5583 & 5589 & & \\ \text { Tcy } & 5487 & 5496 & 5511 & 5583 & 5589 & & \\ \text { W( } & 5445 & 5484 & 5490 & 5610 & 5610 & 5616 & 5616 \\ & 5628 & 5628 & 5631 & 5631 & & & \\ \text { Wr( } & 5445 & 5697 & 5697 & 5697 & 5697 & 5697 & 5697 \\ & 5700 & 5700 & 5700 & 5700 & 5700 & 5700 & \\ \text { X } & 5562 & 5565 & & & & & \\ \text { Y } & 5562 & 5565 & & & & & \end{array}$


$5709 \operatorname{SUB} W(\mathrm{P}(*), \mathrm{Row}, \mathrm{N1}, \mathrm{N} 2, \mathrm{StP}, \mathrm{Wr}(*), \mathrm{W}(*))$ ! - - 81/07/16 -GFE-

5712 FOR $\mathrm{Sp}=1+(\mathrm{N} 1=0)$ TO $1+(\mathrm{N} 2>0)$

5715 CALL Ident $(R 1, \operatorname{Wr}(S p, 1), R 2, \operatorname{Wr}(S p, 2), W 1, \operatorname{Wr}(S p, 3), W 2, \operatorname{Wr}(S p, 4)$, W3, $\operatorname{Wr}(\mathrm{Sp}, 5), \mathrm{L}, \mathrm{L})$

5718 FOR I=1 TO $\mathrm{N} 1 *(\mathrm{Sp}=1)+\mathrm{N} 2 *(\mathrm{Sp}=2)$

! Iterate over either $\mathrm{N} 1$ or $\mathrm{N} 2$

5721 Rw=Row $+(I-1) *$ Stp

$5724 \mathrm{~L}=4 *(\mathrm{Sp}-1) *(\operatorname{COL}(\mathrm{P})=8)$ ! Add 4 to column index as required

$5727 \quad \mathrm{P} 4=\mathrm{P}(\mathrm{Rw}, \mathrm{L}+1) \quad$ ! Read in Pwr values

5730 IF $P 4=0$ THEN Nexti ! If $P 4=0$ no data here, skip to next row

$5733 \mathrm{P} 3=\mathrm{P}(\mathrm{R} w, \mathrm{~L}+2)$

$5736 \quad P 1=R 1 * P(R w, L+3)$

$5739 \mathrm{P} 2=\mathrm{R} 2 * \mathrm{P}(\mathrm{R} w, \mathrm{~L}+4)$

$5742 \mathrm{~S}=(\mathrm{P} 3+\mathrm{P} 4+\mathrm{P} 1 / \mathrm{R} 1+\mathrm{P} 2 / \mathrm{R} 2) / 10$ ! Calc weighting factors

$5745 \mathrm{~S} 3=(\mathrm{P} 3+\mathrm{S})^{\wedge} 2$

$5748 \mathrm{~S} 4=(\mathrm{P} 4+\mathrm{S})^{\wedge} 2$

$5751 \mathrm{~S} 1=(\mathrm{P} 1+\mathrm{S} * \mathrm{R} 1)^{\wedge} 2$

$5754 \mathrm{~S} 2=(\mathrm{P} 2+\mathrm{S} * \mathrm{R} 2)^{\wedge} 2$

$5757 \mathrm{P}=\mathrm{P} 1 * \mathrm{P} 1 / \mathrm{S} 1+\mathrm{P} 2 * \mathrm{P} 2 / \mathrm{S} 2+\mathrm{P} 3 * \mathrm{P} 3 / \mathrm{S} 3+\mathrm{P} 4 * \mathrm{P} 4 / \mathrm{S} 4$

$5760 \mathrm{Q}=\mathrm{P} 1 / \mathrm{S} 1+\mathrm{P} 2 / \mathrm{S} 2+\mathrm{P} 3 / \mathrm{S} 3$

$5763 \mathrm{Wx}=(\mathrm{W} 1+(\mathrm{P} 3-\mathrm{P} 1) / \mathrm{P} 4 / \mathrm{W} 1) / 2$ ! Initial values

$5766 \mathrm{Wy}-((\mathrm{W} 2 * \mathrm{~W} 2+\mathrm{W} 3 * \mathrm{~W} 3+(\mathrm{P} 3-\mathrm{P} 2) / \mathrm{P} 4) / 2-\mathrm{Wx} * \mathrm{~W} 2) / \mathrm{W} 3 !$

5769 GOSUB F ! Calc initial value of " $F$ "

5772 R=1

5775 FOR K=1 TO 30 ! Begin Iteration

$5778 \mathrm{Fa}: \mathrm{Fa}=\mathrm{Fb}$

5781 L $=1$

$5784 \mathrm{Fs}=\mathrm{E} 1 / \mathrm{S} 1+\mathrm{E} 2 / \mathrm{S} 2+\mathrm{E} 3 / \mathrm{S} 3$ ! Begin computation of derivatives

$5787 \mathrm{Fx}=\mathrm{Wx} * \mathrm{Fs}-\mathrm{W} 1 * \mathrm{E} 1 / \mathrm{S} 1-\mathrm{W} 2 * \mathrm{E} 2 / \mathrm{S} 2 \quad !=\mathrm{Fx} / 4$

$5790 \mathrm{Fy}=$ Wy $*$ Fs $-\mathrm{W} 3 * \mathrm{E} 2 / \mathrm{S} 2 \quad !=\mathrm{Fy} / 4$

$5793 \mathrm{D}=\mathrm{Wx} * \mathrm{Q}-\mathrm{W} 1 * \mathrm{P} 1 / \mathrm{S} 1-\mathrm{W} 2 * \mathrm{P} 2 / \mathrm{S} 2$

$5796 \quad E=W y * Q-W 3 * P 2 / S 2$

$5799 \mathrm{C} i=\mathrm{Fs}+2 *\left(\mathrm{Wx} * W x / \mathrm{S} 3+(\mathrm{Wx}-\mathrm{W} 1)^{\wedge} 2 / \mathrm{S} 1+(\mathrm{Wx}-\mathrm{W} 2)^{\wedge} 2 / \mathrm{S} 2-\mathrm{D} * \mathrm{D} / \mathrm{P}\right)$

$5802 \mathrm{Di}=2 *(\mathrm{Wx} * \mathrm{Wy} / \mathrm{S} 3+(\mathrm{Wx}-\mathrm{W} 1) * \mathrm{Wy} / \mathrm{S} 1+(\mathrm{Wx}-\mathrm{W} 2) *(\mathrm{Wy}-\mathrm{W} 3) / \mathrm{S} 2-\mathrm{D} * \mathrm{E} / \mathrm{P})$ !

$5805 \mathrm{Ei}=\mathrm{Fs}+2 *\left(\mathrm{Wy} *(\mathrm{Wy} / \mathrm{S} 3+\mathrm{Wy} / \mathrm{S} 1)+(\mathrm{Wy}-\mathrm{W} 3)^{\wedge} 2 / \mathrm{S} 2-\mathrm{Fx} * \mathrm{E} / \mathrm{P}\right)$

$5808 \quad \mathrm{Zi}=\mathrm{Ci} * \mathrm{Ei}-\mathrm{Di} * \mathrm{Di}$

5811 IF $(\mathrm{Zi}<0)$ OR $(\mathrm{E} i<0)$ THEN Bkup

$5814 \mathrm{X}=(\mathrm{Fx} * \mathrm{Ei}-\mathrm{Fy} * \mathrm{Di}) / \mathrm{Zi}$

! $=$ Fyy $/ 4$

$5817 \quad \mathrm{Y}=(\mathrm{Fy} * \mathrm{C} \mathrm{i}-\mathrm{Fx} * \mathrm{Di}) / \mathrm{Zi}$

5820 CALL Csub (Wx, Wy, X, Y,Wx, Wy)

5823 GOSUB F

5826 IF $\mathrm{Fb}<\mathrm{Fa}+1 \mathrm{E}-10$ THEN ON $1+(\mathrm{L}=1) *(\mathrm{X} * \mathrm{X}+\mathrm{Y} * \mathrm{Y}<1 \mathrm{E}-12)$ GoTo Nextk, Exit

$5829 \mathrm{~L}=2 * \mathrm{~L} \quad$ ! Routine to reduce step size

5832 IF L-128 THEN Bkup2 ! Go to "backup routine"

5835 CALL Cadd(Wx, Wy, X/L, Y/L, Wx, Wy)! Restore a fraction of step

5838 GOTO 5823

5841 Nextk: NEXT K

5844 Error: PRINT "Abnormal-exit from circle routine "; "Sp- "; $\mathrm{Sp}$, , Row = ";Rw

5847 PRINT "Circle residual= "; $\mathrm{Pi}$ SQR $(\mathrm{Fb}) / 2$ 
5850 Exit: $\mathrm{L}-4 *(\mathrm{Sp}=2) *(\operatorname{COL}(\mathrm{W})-8)$

5853

$W(I, 1+L)=W x$

5856

$W(I, 2+L)=W y$

5859

$W(I, 3+L)=P i$

5862

$W(I, 4+L)=P i * S Q R(F b) / 2$

5865 Nexti: NEXT I

5868 NEXT Sp

5871 SUBEXIT

$5874 !$

5877 Bkup2: CALL Cadd(Wx,Wy,X/64, Y/64,Wx, Wy)

5880 ! Backup algorithm based on Goldfeld, etal, Econometrica, July 1966, pg 541

5883 Bkup: $E g=\left(C i+E i-S Q R\left((C i-E i)^{\wedge} 2+4 * D i * D i\right)\right) / 2 \quad !$ (Smallest

5886 IF $\mathrm{Fx} * \mathrm{Fx}+\mathrm{Fy} * \mathrm{Fy}>1 \mathrm{E}-12$ THEN Ka eigenvalue) $/ 4$

$5889 \mathrm{Ri}=.1 * \mathrm{SQR}(\mathrm{P} 3 *(\mathrm{P} 3<\mathrm{P} 1) *(\mathrm{P} 3<\mathrm{P} 2)+\mathrm{P} 1 *(\mathrm{P} 1<\mathrm{P} 3) *(\mathrm{P} 1<\mathrm{P} 2)+\mathrm{P} 2 *(\mathrm{P} 2<\mathrm{P} 1)$ $*(\mathrm{P} 2<\mathrm{P} 3))$

$5892 \mathrm{X}=-\mathrm{R} i * \mathrm{Di} / \mathrm{SQR}\left(\mathrm{Di} * \mathrm{Di}+(\mathrm{Ci}-\mathrm{Eg})^{\wedge} 2\right)$

$5895 \mathrm{Y}=\mathrm{Ri} *(\mathrm{Ci}-\mathrm{Eg}) / \mathrm{SQR}\left(\mathrm{Di} * \mathrm{Di}+(\mathrm{Ci}-\mathrm{Eg})^{\wedge} 2\right)$

5898 CALL Csub(Wx, Wy, X, Y, Wx, Wy)

5901 GOSUB F

5904 GOTO Fa

$5907 \mathrm{Ka}:$ FOR $\mathrm{Ka}=1$ TO 20

$5910 \quad \mathrm{Al}=\mathrm{Eg}-\mathrm{R} * \mathrm{SQR}(\mathrm{Fx} * \mathrm{Fx}+\mathrm{Fy} * \mathrm{Fy})$ ! Correction to $\mathrm{Fxx} \& \mathrm{Fyy}$

5913 CALL Csub(Ci,Ei, Al, Al, Ca, Ea)

$5916 \quad \mathrm{Za}=\mathrm{Ca} * \mathrm{Ea}-\mathrm{Di} * \mathrm{Di}$

$5919 \mathrm{X}=(\mathrm{Fx} * \mathrm{Ea}-\mathrm{Fy} * \mathrm{Di}) / \mathrm{Za}$

$5922 \quad \mathrm{Y}=(\mathrm{Fy} * \mathrm{Ca}-\mathrm{Fx} * \mathrm{Di}) / \mathrm{Za}$

5925 CALL Csub(Wx,Wy, X, Y,Wx, Wy)

5928 GOSUB F

$5931 \mathrm{Zc}=(\mathrm{Fb}-\mathrm{Fa}) /(2 *(\mathrm{C} i * \mathrm{X} * \mathrm{X}+2 * \mathrm{Di} * \mathrm{X} * \mathrm{Y}+\mathrm{E} i * \mathrm{Y} * \mathrm{Y})-4 *(\mathrm{Fx} * \mathrm{X}+\mathrm{Fy} * \mathrm{Y}))-1$

5934 IF $\mathrm{Fb}>\mathrm{Fa}+1 \mathrm{E}-10$ THEN $\mathrm{Zc}=-2$ ! This insures a reduced step size

$5937 \quad R=R *(.4+3.6 *(A B S(Z c)>.3) *(1-(A B S(Z c)<1) *(1-A B S(Z c)) / .7))$

5940 IF $Z$ C >-1 THEN 5841

5943 CALL Cadd(Wx, Wy, X,Y,Wx, Wy)! Returns to original position and

5946 NEXT Ka ! repeats with smaller step size

5949 GOTO Error

$5952 \mathrm{~F}: \mathrm{Wm}=\mathrm{Wx} * W x+W y * W y \quad$ ! Compute value of "F" $(=\mathrm{Fb})$

$5955 \mathrm{Wlm}=\mathrm{Wm}+\mathrm{Wl} *(\mathrm{~W} 1-2 * W \mathrm{~W})$

$5958 W 2 m=W m+W 2 *(W 2-2 * W x)+W 3 *(W 3-2 * W y)$

$5961 \mathrm{Pi}=\mathrm{P} /(\mathrm{Wm} * \mathrm{P} 3 / \mathrm{S} 3+\mathrm{P} 4 / \mathrm{S} 4+\mathrm{W} 1 \mathrm{~m} * \mathrm{P} 1 / \mathrm{S} 1+\mathrm{W} 2 \mathrm{~m} * \mathrm{P} 2 / \mathrm{S} 2)$

5964 E3=Wm-P3/Pi

$5967 \mathrm{E} 1=\mathrm{W} 1 \mathrm{~m}-\mathrm{P} 1 / \mathrm{Pi}$

5970 E2 $=W 2 \mathrm{~m}-\mathrm{P} 2 / \mathrm{Pi}$

$5973 \mathrm{Fb}=\mathrm{E} 1 * \mathrm{E} 1 / \mathrm{S} 1+\mathrm{E} 2 * \mathrm{E} 2 / \mathrm{S} 2+\mathrm{E} 3 * \mathrm{E} 3 / \mathrm{S} 3+(1-\mathrm{P} 4 / \mathrm{P} 1)^{\wedge} 2 / \mathrm{S} 4$

5976 RETURN

5979 SUBEND

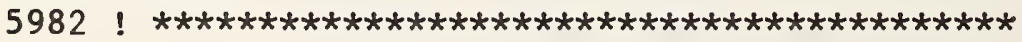

S W

A1 $5910 \quad 5913 \quad 5913$

Bkup2: $\quad 5877 \quad 5832$ 


\begin{tabular}{|c|c|c|c|c|c|c|c|}
\hline Bkup: & 5883 & 5811 & & & & & \\
\hline $\mathrm{Ca}$ & 5913 & 5916 & 5922 & & & & \\
\hline $\mathrm{CI}$ & $\begin{array}{l}5799 \\
5895\end{array}$ & $\begin{array}{l}5808 \\
5913\end{array}$ & $\begin{array}{l}5817 \\
5931\end{array}$ & 5883 & 5883 & 5892 & 5895 \\
\hline D & 5793 & 5799 & 5799 & 5802 & & & \\
\hline $\mathrm{Di}$ & $\begin{array}{l}5802 \\
5892 \\
5919\end{array}$ & $\begin{array}{l}5808 \\
5892 \\
5922\end{array}$ & $\begin{array}{l}5808 \\
5892 \\
5931\end{array}$ & $\begin{array}{l}5814 \\
5895 \\
5796\end{array}$ & $\begin{array}{l}5817 \\
5895 \\
5802\end{array}$ & $\begin{array}{l}5883 \\
5916 \\
5805\end{array}$ & $\begin{array}{l}5883 \\
5916 \\
5805\end{array}$ \\
\hline E1 & 5784 & 5787 & 5967 & 5973 & 5973 & & \\
\hline E2 & 5784 & 5787 & 5790 & 5970 & 5973 & 5973 & \\
\hline E3 & 5784 & 5964 & 5973 & 5973 & & & \\
\hline $\mathrm{Ea}$ & 5913 & 5916 & 5919 & & & & \\
\hline Eg & 5883 & 5892 & 5895 & 5895 & 5910 & & \\
\hline Ei & $\begin{array}{l}5805 \\
5931\end{array}$ & 5808 & 5811 & 5814 & 5883 & 5883 & 5913 \\
\hline Error: & 5844 & 5949 & & & & & \\
\hline Exit: & 5850 & 5826 & & & & & \\
\hline F: & 5952 & 5769 & 5823 & 5901 & 5928 & & \\
\hline $\mathrm{Fa}$ & 5778 & 5826 & 5931 & 5934 & & & \\
\hline $\mathrm{Fa}:$ & 5778 & 5904 & & & & & \\
\hline $\mathrm{Fb}$ & 5778 & 5826 & 5847 & 5862 & 5931 & 5934 & 5973 \\
\hline Fs & 5784 & 5787 & 5790 & 5799 & 5805 & & \\
\hline$F x$ & $\begin{array}{l}5787 \\
5919\end{array}$ & $\begin{array}{l}5814 \\
5922\end{array}$ & $\begin{array}{l}5817 \\
5931\end{array}$ & 5886 & 5886 & 5910 & 5910 \\
\hline Fy & $\begin{array}{l}5790 \\
5919\end{array}$ & $\begin{array}{l}5814 \\
5922\end{array}$ & $\begin{array}{l}5817 \\
5931\end{array}$ & 5886 & 5886 & 5910 & 5910 \\
\hline I & 5718 & 5721 & 5853 & 5856 & 5859 & 5862 & 5865 \\
\hline K & 5775 & 5841 & & & & & \\
\hline $\mathrm{Ka}$ & 5907 & 5946 & & & & & \\
\hline $\mathrm{Ka}:$ & 5907 & 5886 & & & & & \\
\hline
\end{tabular}




\begin{tabular}{|c|c|c|c|c|c|c|c|}
\hline L & $\begin{array}{l}5715 \\
5781 \\
5850\end{array}$ & $\begin{array}{l}5715 \\
5826 \\
5853\end{array}$ & $\begin{array}{l}5724 \\
5829 \\
5856\end{array}$ & $\begin{array}{l}5727 \\
5829 \\
5859\end{array}$ & $\begin{array}{l}5733 \\
5832 \\
5862\end{array}$ & $\begin{array}{l}5736 \\
5835\end{array}$ & $\begin{array}{l}5739 \\
5835\end{array}$ \\
\hline N1 & 5709 & 5712 & 5718 & & & & \\
\hline N2 & 5709 & 5712 & 5718 & & & & \\
\hline Nexti: & 5865 & 5730 & & & & & \\
\hline Nextk: & 5841 & 5826 & & & & & \\
\hline $\mathbf{P}$ & 5757 & 5799 & 5802 & 5805 & 5961 & & \\
\hline $\mathbf{P}($ & 5709 & 5724 & 5727 & 5733 & 5736 & 5739 & \\
\hline P1 & $\begin{array}{l}5736 \\
5793 \\
5967\end{array}$ & $\begin{array}{l}5742 \\
5889\end{array}$ & $\begin{array}{l}5751 \\
5889\end{array}$ & $\begin{array}{l}5757 \\
5889\end{array}$ & $\begin{array}{l}5757 \\
5889\end{array}$ & $\begin{array}{l}5760 \\
5889\end{array}$ & $\begin{array}{l}5763 \\
5961\end{array}$ \\
\hline P2 & $\begin{array}{l}5739 \\
5793 \\
5961\end{array}$ & $\begin{array}{l}5742 \\
5796 \\
5970\end{array}$ & $\begin{array}{l}5754 \\
5889\end{array}$ & $\begin{array}{l}5757 \\
5889\end{array}$ & $\begin{array}{l}5757 \\
5889\end{array}$ & $\begin{array}{l}5760 \\
5889\end{array}$ & $\begin{array}{l}5766 \\
5889\end{array}$ \\
\hline P3 & $\begin{array}{l}5733 \\
5766 \\
5964\end{array}$ & $\begin{array}{l}5742 \\
5889\end{array}$ & $\begin{array}{l}5745 \\
5889\end{array}$ & $\begin{array}{l}5757 \\
5889\end{array}$ & $\begin{array}{l}5757 \\
5889\end{array}$ & $\begin{array}{l}5760 \\
5889\end{array}$ & $\begin{array}{l}5763 \\
5961\end{array}$ \\
\hline P4 & $\begin{array}{l}5727 \\
5766\end{array}$ & $\begin{array}{l}5730 \\
5961\end{array}$ & $\begin{array}{l}5742 \\
5973\end{array}$ & 5748 & 5757 & 5757 & 5763 \\
\hline $\mathrm{Pi}$ & $\begin{array}{l}5847 \\
5973\end{array}$ & 5859 & 5862 & 5961 & 5964 & 5967 & 5970 \\
\hline Q & 5760 & 5793 & 5796 & & & & \\
\hline $\mathbf{R}$ & 5772 & 5910 & 5937 & 5937 & & & \\
\hline R1 & 5715 & 5736 & 5742 & 5751 & & & \\
\hline R2 & 5715 & 5739 & 5742 & 5754 & & & \\
\hline $\mathrm{Ri}$ & 5889 & 5892 & 5895 & & & & \\
\hline Row & 5709 & 5721 & & & & & \\
\hline$R w$ & 5721 & 5727 & 5733 & 5736 & 5739 & 5844 & \\
\hline$S$ & 5742 & 5745 & 5748 & 5751 & 5754 & & \\
\hline S1 & $\begin{array}{l}5751 \\
5802\end{array}$ & $\begin{array}{l}5757 \\
5805\end{array}$ & $\begin{array}{l}5760 \\
5961\end{array}$ & $\begin{array}{l}5784 \\
5973\end{array}$ & 5787 & 5793 & 5799 \\
\hline s2 & $\begin{array}{l}5754 \\
5796\end{array}$ & $\begin{array}{l}5757 \\
5799\end{array}$ & $\begin{array}{l}5760 \\
5802\end{array}$ & $\begin{array}{l}5784 \\
5805\end{array}$ & $\begin{array}{l}5787 \\
5961\end{array}$ & $\begin{array}{l}5790 \\
5973\end{array}$ & 5793 \\
\hline
\end{tabular}


$5961 \quad 5973$

54

$\begin{array}{llll}5748 & 5757 \quad 5961 & 5973\end{array}$

$\mathrm{Sp}$

$\begin{array}{lllllll}5712 & 5715 & 5715 & 5715 & 5715 & 5715 & 5718\end{array}$

Stp

$\begin{array}{lllll}5718 & 5724 & 5844 & 5850 \quad 5868\end{array}$

WC

W1

$5709 \quad 5721$

$\begin{array}{llllll}5709 & 5850 & 5853 & 5856 & 5859 & 5862\end{array}$

$\begin{array}{lllllll}5715 & 5763 & 5763 & 5787 & 5793 & 5799 & 5802\end{array}$

$5955 \quad 5955$

W1m

$5955 \quad 5961 \quad 5967$

W2

$\begin{array}{lllllll}5715 & 5766 & 5766 & 5766 & 5787 & 5793 & 5799 \\ 5802 & 5958 & 5958 & & & & \end{array}$

W $2 \mathrm{~m}$

$5958 \quad 5961 \quad 5970$

W3

$\begin{array}{lllllll}5715 & 5766 & 5766 & 5766 & 5790 & 5796 & 5802\end{array}$

$5805 \quad 5958 \quad 5958$

$\mathrm{Wm}$

$\begin{array}{lllll}5952 & 5955 \quad 5958 & 5961 & 5964\end{array}$

$\operatorname{Wr}($

$\begin{array}{llllll}5709 & 5715 \quad 5715 & 5715 & 5715 & 5715\end{array}$

Wx

$\begin{array}{lllllll}5763 & 5766 & 5787 & 5793 & 5799 & 5799 & 5799 \\ 5799 & 5802 & 5802 & 5802 & 5820 & 5820 & 5835 \\ 5835 & 5853 & 5877 & 5877 & 5898 & 5898 & 5925 \\ 5925 & 5943 & 5943 & 5952 & 5952 & 5955 & 5958\end{array}$

Wy

$\begin{array}{lllllll}5766 & 5790 & 5796 & 5802 & 5802 & 5802 & 5805\end{array}$

$\begin{array}{lllllll}5805 & 5805 & 5805 & 5820 & 5820 & 5835 & 5835\end{array}$

$\begin{array}{lllllll}5856 & 5877 & 5877 & 5898 & 5898 & 5925 & 5925\end{array}$

$\begin{array}{llll}5943 & 5943 \quad 5952 \quad 5952 \quad 5958\end{array}$

X

$\begin{array}{lllllll}5814 & 5820 & 5826 & 5826 & 5835 & 5877 & 5892 \\ 5898 & 5919 & 5925 & 5931 & 5931 & 5931 & 5931\end{array}$

5943

Y

$\begin{array}{lllllll}5817 & 5820 & 5826 & 5826 & 5835 & 5877 & 5895\end{array}$

$\begin{array}{lllllll}5898 & 5922 & 5925 & 5931 & 5931 & 5931 & 5931\end{array}$

5943

$\mathrm{Za}$

$5916 \quad 5919 \quad 5922$

Zc

$\begin{array}{llllll}5931 & 5934 & 5937 & 5937 & 5937 & 5940\end{array}$

$\mathrm{Zi}$

$5808 \quad 5811 \quad 5814 \quad 5817$ 
5985 SUB Rtpt(G(*), Row, S(*),R) ! - (81/05/11)-GFE-- -

5988 ! Compute two-port cascading parameters ( $\mathrm{Ax}, \mathrm{Bx}, \mathrm{Cx}, \&$ Ay, By, Cy )$=S(1) \cdots S(6)$

5991 DIM $B(1: 8,1: 6), X(1: 8), Y(1: 8)$

$5994 \mathrm{~L}=2 *(\operatorname{COL}(G)>4)$ ! Adjustment for two different formats for " $G$ " 5997 FOR $I=1$ TO 4

$6000 \mathrm{~J}=\mathrm{I}+4$

$6003 \mathrm{~K}=\mathrm{I}+$ Row -1

$6006 \mathrm{~B}(\mathrm{I}, 1)=\mathrm{B}(\mathrm{J}, 4)=1$

$6009 B(I, 2)=B(J, 5)=C=G(K, 3+L)$

$6012 B(I, 3)=B(J, 6)=A=-G(K, 1)$

$6015 B(I, 4)=B(J, 1)=0$

$6018 B(I, 5)=D=-G(K, 4+L)$

$6021 B(I, 6)=B=G(K, 2)$

$6024 B(J, 2)=-D$

$6027 B(J, 3)=-B$

6030 CALL Cmpy (-A, B, C, -D,Y(I),Y(J))

6033 NEXT I

6036 CALL Msoln(B(*), $Y(*), S(*))$

6039 MAT $X=B * S$

6042 MAT $X=Y-X$

$6045 \mathrm{R}=\mathrm{SQR}(\mathrm{DOT}(\mathrm{X}, \mathrm{X}) / \mathrm{DOT}(\mathrm{S}, \mathrm{S}))$

6048 SUBEND

6051 ! **********************************

S RtptC

A

B

BC

C

D

G(

I

$\mathrm{J}$

K

L

R

Row

60126030

$6021 \quad 6027 \quad 6030$

$\begin{array}{lllllll}5991 & 6006 & 6006 & 6009 & 6009 & 6012 & 6012\end{array}$

$\begin{array}{lllllll}6015 & 6015 & 6018 & 6021 & 6024 & 6027 & 6036\end{array}$

6039

60096030

$6018 \quad 6024 \quad 6030$

$\begin{array}{llllll}5985 & 5994 & 6009 & 6012 & 6018 & 6021\end{array}$

$\begin{array}{lllllll}5997 & 6000 & 6003 & 6006 & 6009 & 6012 & 6015\end{array}$

$\begin{array}{llll}6018 & 6021 & 6030 & 6033\end{array}$

$\begin{array}{lllllll}6000 & 6006 & 6009 & 6012 & 6015 & 6024 & 6027\end{array}$

6030

$\begin{array}{lllll}6003 & 6009 & 6012 & 6018 & 6021\end{array}$

$5994 \quad 6009 \quad 6018$

$5985 \quad 6045$

$5985 \quad 6003$ 
6045

S(

$\begin{array}{llll}5985 & 6036 \quad 6039 \quad 6045\end{array}$

X

6045

$\begin{array}{lllll}5991 & 6039 & 6042 & 6042 & 6045\end{array}$

$\begin{array}{lllll}5991 & 6030 & 6030 & 6036 & 6042\end{array}$ 
6054 SUB Msoln $(B(*), Y(*), X(*)) !-(80 / 1 / 16)$

6057 OPTION BASE 1

6060 DIM A $(9,9), C(9,16), U(9)$

$6063 \mathrm{M}=\mathrm{ROW}(\mathrm{B})$

$6066 \mathrm{~N}=\mathrm{COL}(\mathrm{B})$

$6069 \operatorname{REDIM~} A(\mathrm{~N}, \mathrm{~N}), \mathrm{C}(\mathrm{N}, \mathrm{M}), \mathrm{U}(\mathrm{N})$

6072 MAT C=TRN(B)

6075 MAT $A=C * B$

6078 MAT $A=\operatorname{INV}(A)$

6081 MAT $U=C * Y$

6084 MAT $\mathrm{X}=\mathrm{A} * \mathrm{U}$

6087 SUBEND

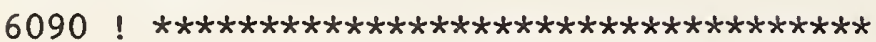

S Msoln(

$\begin{array}{lllllll}\text { AC } & 6060 & 6069 & 6075 & 6078 & 6078 & 6084 \\ \text { B( } & 6054 & 6063 & 6066 & 6072 & 6075 \\ \text { C } & 6060 & 6069 & 6072 & 6075 & 6081 \\ \text { M } & 6063 & 6069 & & & \\ \text { N } & 6066 & 6069 & 6069 & 6069 & 6069 \\ \text { U( } & 6060 & 6069 & 6081 & 6084 & \\ X( & 6054 & 6084 & & & \\ Y( & 6054 & 6081 & & & \end{array}$




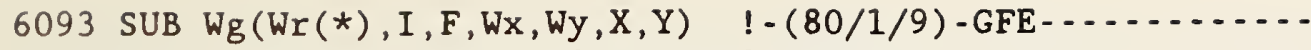

$6096 \operatorname{CALL} \operatorname{Csub}(\mathrm{Wx}, \operatorname{Wy}, \operatorname{Wr}(I, 8), \operatorname{Wr}(I, 9), \mathrm{Nx}, \mathrm{Ny})$

6099 CALL Cmpy(Wr(I, 10), Wr (I, 11), Wx, Wy, X, Y)

$6102 \operatorname{CALL} \operatorname{Csub}(\operatorname{Wr}(I, 6), \operatorname{Wr}(I, 7), X, Y, D x, D y)$

6105 CALL Cdiv(Nx, Ny, Dx, Dy, X, Y)

6108 IF F-1 THEN CALL Polar(X,Y,X,Y)

6111 SUBEND

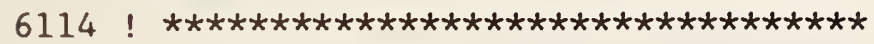

$\mathrm{S} \mathrm{Wg}($

Dx

61026105

Dy

61026105

F

60936108

I

$\begin{array}{lllllll}6093 & 6096 & 6096 & 6099 & 6099 & 6102 & 6102\end{array}$

$\mathrm{Nx}$

$6096 \quad 6105$

Ny

$6096 \quad 6105$

Wr (

$\begin{array}{lllllll}6093 & 6096 & 6096 & 6099 & 6099 & 6102 & 6102\end{array}$

Wx

$60936096 \quad 6099$

Wy

$60936096 \quad 6099$

$\mathrm{X}$

$\begin{array}{llllll}6093 & 6099 & 6102 & 6105 & 6108 & 6108\end{array}$

Y

$\begin{array}{llllll}6093 & 6099 & 6102 & 6105 & 6108 & 6108\end{array}$ 
6117 SUB Wq(W(*),Q(*)) ! - $\mathrm{W0} / 04 / 03 \ldots$. GFE $\ldots . . . . . . . . .$.

6120 FOR I 1 TO ROW(W)

6123 CALL Ident $(A, W(I, 1), B, W(I, 2), C, W(I, 3), D, W(I, 4), E, W(I, 5), F$, $W(I, 6))$

6126 CALL Ident $(G, W(I, 7), H, W(I, 8), P, W(I, 9), Q, W(I, 10), R, W(I, 11)$, $Q(I, 12), W(I, 12))$

6129 CALL Polar $(-Q,-R, Q(I, 1), Q(I, 2))$

$6132 \mathrm{Q}(I, 9)=S=S Q R(F * F+G * G)$

6135 CALL Polar $((-F * H-G * P) / S / S,(G * H-F * P) / S / S, Q(I, 3), Q(I, 4))$

6138 CALL $W g(W(*), I, 1, C, 0, Q(I, 5), Q(I, 6))$

$6141 \operatorname{CALL} W g(W(*), I, 1, D, E, Q(I, 7), Q(I, 8))$

$6144 \mathrm{Q}(\mathrm{I}, 10)-\mathrm{SQR}\left(\left((\mathrm{F}-\mathrm{C} * \mathrm{Q})^{\wedge} 2+(\mathrm{G}-\mathrm{C} * \mathrm{R})^{\wedge} 2\right) / \mathrm{A}\right)$

$6147 \mathrm{Q}(I, 11)=\mathrm{SQR}\left(\left((F-D * Q+E * R)^{\wedge} 2+(G-D * R-E * Q) \wedge 2\right) / B\right)$

6150 NEXT I

6153 SUBEND

6156 ! $* * * * * * * * * * * * * * * * * * * * * * * * * * * * * *$

S Wq

\begin{tabular}{|c|c|c|c|c|c|c|c|}
\hline A & 6123 & 6144 & & & & & \\
\hline B & 6123 & 6147 & & & & & \\
\hline C & 6123 & 6138 & 6144 & 6144 & & & \\
\hline D & 6123 & 6141 & 6147 & 6147 & & & \\
\hline$E$ & 6123 & 6141 & 6147 & 6147 & & & \\
\hline$F$ & 6123 & 6132 & 6132 & 6135 & 6135 & 6144 & 6147 \\
\hline G & 6126 & 6132 & 6132 & 6135 & 6135 & 6144 & 6147 \\
\hline $\mathrm{H}$ & 6126 & 6135 & 6135 & & & & \\
\hline I & $\begin{array}{l}6120 \\
6126 \\
6129 \\
6138\end{array}$ & $\begin{array}{l}6123 \\
6126 \\
6129 \\
6141\end{array}$ & $\begin{array}{l}6123 \\
6126 \\
6132 \\
6141\end{array}$ & $\begin{array}{l}6123 \\
6126 \\
6135 \\
6141\end{array}$ & $\begin{array}{l}6123 \\
6126 \\
6135 \\
6144\end{array}$ & $\begin{array}{l}6123 \\
6126 \\
6138 \\
6147\end{array}$ & $\begin{array}{l}6123 \\
6126 \\
6138 \\
6150\end{array}$ \\
\hline$P$ & 6126 & 6135 & 6135 & & & & \\
\hline$Q$ & 6126 & 6129 & 6144 & 6147 & 6147 & & \\
\hline Q ( & $\begin{array}{l}6117 \\
6138\end{array}$ & $\begin{array}{l}6126 \\
6138\end{array}$ & $\begin{array}{l}6129 \\
6141\end{array}$ & $\begin{array}{l}6129 \\
6141\end{array}$ & $\begin{array}{l}6132 \\
6144\end{array}$ & $\begin{array}{l}6135 \\
6147\end{array}$ & 6135 \\
\hline $\mathbf{R}$ & 6126 & 6129 & 6144 & 6147 & 6147 & & \\
\hline$S$ & 6132 & 6135 & 6135 & 6135 & 6135 & & \\
\hline W( & $\begin{array}{l}6117 \\
6123 \\
6138\end{array}$ & $\begin{array}{l}6120 \\
6126 \\
6141\end{array}$ & $\begin{array}{l}6123 \\
6126\end{array}$ & $\begin{array}{l}6123 \\
6126\end{array}$ & $\begin{array}{l}6123 \\
6126\end{array}$ & $\begin{array}{l}6123 \\
6126\end{array}$ & $\begin{array}{l}6123 \\
6126\end{array}$ \\
\hline
\end{tabular}


$6159 \operatorname{SUB} \operatorname{Mg}(\mathrm{P}(*), \operatorname{Row}, \mathrm{SP}, \operatorname{Wr}(*), F \$, X, Y, \operatorname{Res}) \quad$ ! - $(81 / 07 / 16)-\mathrm{GFE}-\cdot$

6162 DIM W(1:1,1:4)

6165 CALL $W(P(*)$, Row, $S p=1, S p=2,1, W r(*), W(*))$

6168 CALL $W g\left(W r(*), S_{P}, P, W(1,1), W(1,2), X, Y\right)$

6171 IF F\$-"P" THEN CALL Polar $(X, Y, X, Y)$

6174 Res-W $(1,4)$

6177 SUBEND

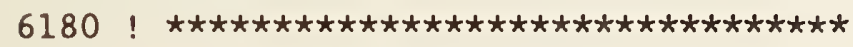

$\mathrm{S} \mathrm{Mg}($

$F \$$

$6159 \quad 6171$

$P$

6168

P(

$6159 \quad 6165$

Res

$6159 \quad 6174$

Row

61596165

$\mathrm{sp}$

$\begin{array}{llll}6159 & 6165 \quad 6165 \quad 6168\end{array}$

W(

$\begin{array}{lllll}6162 & 6165 & 6168 & 6168 & 6174\end{array}$

$\operatorname{Wr}($

$\begin{array}{lll}6159 & 6165 \quad 6168\end{array}$

$\mathrm{X}$

$\begin{array}{llll}6159 & 6168 & 6171 & 6171\end{array}$

Y

$\begin{array}{llll}6159 & 6168 & 6171 & 6171\end{array}$ 
6183 SUB Cadd (Ax, Ay, Bx, By, Cx, Cy) !-(80/1/16) - -GFE---

$6186 \mathrm{Cx}=\mathrm{Ax}+\mathrm{Bx}$

6189 Cy=Ay+By

6192 SUBEND

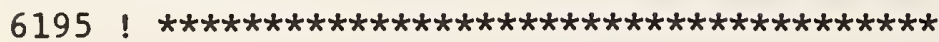

S Cadd

Ax

Ay

Bx

By

$\mathrm{Cx}$

Cy
$6183 \quad 6186$

$6183 \quad 6189$

$6183 \quad 6186$

$6183 \quad 6189$

$6183 \quad 6186$

$6183 \quad 6189$ 
6198 SUB $C$ sub $(A x, A y, B x, B y, C x, C y) !-(80 / 1 / 16) \ldots . . . .-G F E-\cdot$

$6201 \mathrm{Cx}-\mathrm{Ax}-\mathrm{Bx}$

6204 Cy-Ay-By

6207 SUBEND

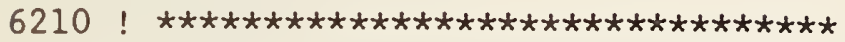

S Csub(

Ax

Ay

$\mathrm{Bx}$

By

$\mathrm{Cx}$

Cy
$6198 \quad 6201$

$6198 \quad 6204$

$6198 \quad 6201$

$6198 \quad 6204$

$6198 \quad 6201$

$6198 \quad 6204$ 
6213 SUB Cmpy (Ax, Ay, Bx, By, Cx, Cy) ! - (80/1/16)-GFE--.-

6216 CALL Ident ( $X, A x, Y, A y, U, B x, V, B y, A, 0, B, 0)$

$6219 \mathrm{CX}=\mathrm{X} * \mathrm{U}-\mathrm{Y} * \mathrm{~V}$

$6222 \mathrm{Cy}=\mathrm{X} * \mathrm{~V}+\mathrm{Y} * \mathrm{U}$

6225 SUBEND

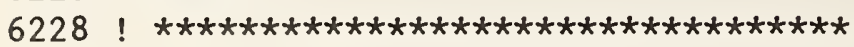

S Cmpyl

\begin{tabular}{|c|c|c|c|}
\hline A & 6216 & & \\
\hline$A x$ & 6213 & 6216 & \\
\hline Ay & 6213 & 6216 & \\
\hline B & 6216 & & \\
\hline$B x$ & 6213 & 6216 & \\
\hline By & 6213 & 6216 & \\
\hline $\mathrm{Cx}$ & 6213 & 6219 & \\
\hline Cy & 6213 & 6222 & \\
\hline $\mathrm{U}$ & 6216 & 6219 & 6222 \\
\hline V & 6216 & 6219 & 6222 \\
\hline $\mathrm{X}$ & 6216 & 6219 & 6222 \\
\hline$Y$ & 6216 & 6219 & 6222 \\
\hline
\end{tabular}




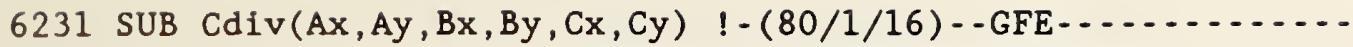

6234 CALL Ident $(X, A x, Y, A y, U, B x, V, B y, A, B x * B x+B y * B y, B, 0)$

$6237 \mathrm{CX}-(\mathrm{X} * \mathrm{U}+\mathrm{Y} * \mathrm{~V}) / \mathrm{A}$

$6240 \mathrm{Cy}=(\mathrm{Y} * \mathrm{U}-\mathrm{X} * \mathrm{~V}) / \mathrm{A}$

6243 SUBEND

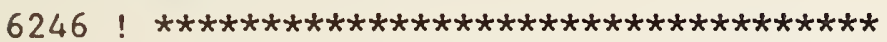

\section{S Cdiv(}

$\begin{array}{lllll}\text { A } & 6234 & 6237 & 6240 & \\ \text { Ax } & 6231 & 6234 & & \\ \text { Ay } & 6231 & 6234 & & \\ \text { B } & 6234 & & & \\ \text { Bx } & 6231 & 6234 & 6234 & 6234 \\ \text { By } & 6231 & 6234 & 6234 & 6234 \\ \text { CX } & 6231 & 6237 & & \\ \text { Cy } & 6231 & 6240 & & \\ \text { U } & 6234 & 6237 & 6240 & \\ \text { V } & 6234 & 6237 & 6240 & \\ \text { X } & 6234 & 6237 & 6240 & \\ \text { Y } & 6234 & 6237 & 6240 & \end{array}$




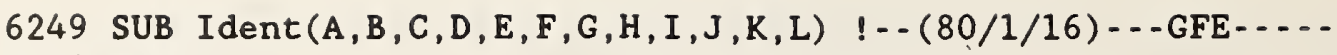

6252 A=B

$6255 \mathrm{C}=\mathrm{D}$

$6258 \quad \mathrm{E}=\mathrm{F}$

$6261 \mathrm{G}=\mathrm{H}$

$6264 \mathrm{I}=\mathrm{J}$

$6267 \mathrm{~K}=\mathrm{L}$

6270 SUBEND

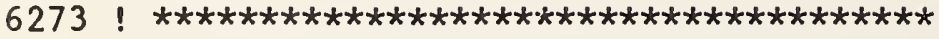

S Ident(

A

$6249 \quad 6252$

B

$6249 \quad 6252$

C

$6249 \quad 6255$

D

$6249 \quad 6255$

E

$6249 \quad 6258$

F

$6249 \quad 6258$

G

$6249 \quad 6261$

H

$6249 \quad 6261$

I

$6249 \quad 6264$

J

62496264

K

$6249 \quad 6267$

L

$6249 \quad 6267$ 


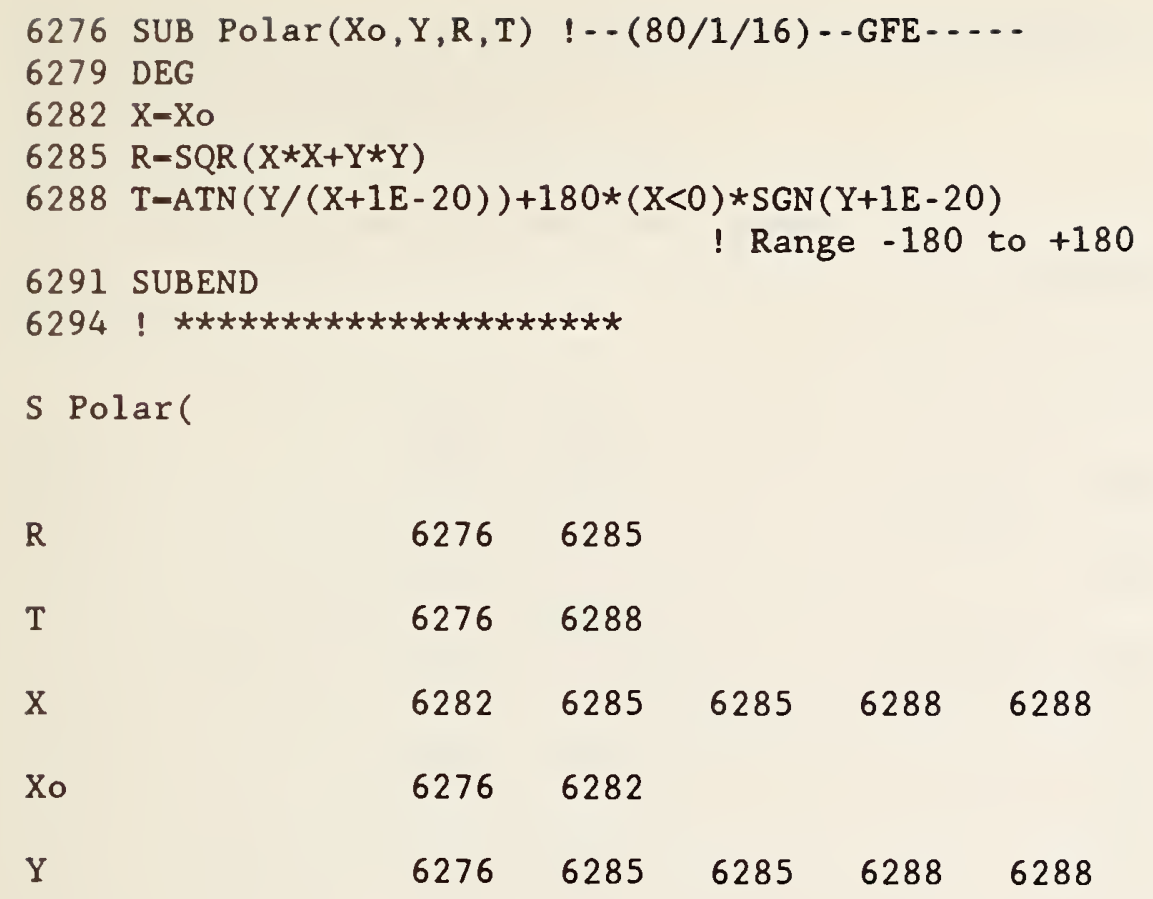




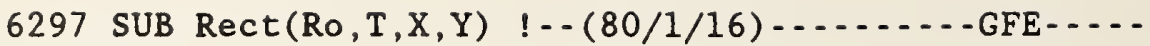

6300 DEG

6303 R=Ro

$6306 \mathrm{X}=\mathrm{R} * \operatorname{COS}(\mathrm{T})$

$6309 \mathrm{Y}=\mathrm{R} * \mathrm{SIN}(\mathrm{T})$

6312 SUBEND

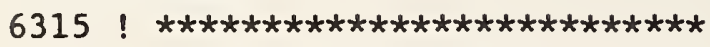

S Rect (

$\begin{array}{llll}\text { R } & 6303 & 6306 & 6309 \\ \text { Ro } & 6297 & 6303 & \\ \text { T } & 6297 & 6306 & 6309 \\ \mathrm{X} & 6297 & 6306 & \\ \mathrm{Y} & 6297 & 6309 & \end{array}$


6318 SUB Cpwr(Pstd, Pwr(*), Row, Sp, Wr(*), Res) !--(81/05/06)-GFE---

6321 CALL Mpwr(Pwr(*), Row, Sp, Wr (*), "N", X, Y, "Net", Pnet, Res)

$6324 \operatorname{Wr}(1,12)-$ Pstd/Pnet

$6327 \operatorname{Wr}(2,12)-\operatorname{Wr}(2,12) * \operatorname{Wr}(1,12)$

6330 SUBEND

6333 ! $* * * * * * * * * * * * * * * * * * * * * * * * * * * * * *$

S Cpwr(

\begin{tabular}{|c|c|c|c|c|c|c|}
\hline Pnet & 6321 & 6324 & & & & \\
\hline Pstd & 6318 & 6324 & & & & \\
\hline Pwr ( & 6318 & 6321 & & & & \\
\hline Res & 6318 & 6321 & & & & \\
\hline Row & 6318 & 6321 & & & & \\
\hline$S p$ & 6318 & 6321 & & & & \\
\hline Wr( & 6318 & 6321 & 6324 & 6327 & 6327 & 6327 \\
\hline $\mathrm{X}$ & 6321 & & & & & \\
\hline $\mathrm{Y}$ & 6321 & & & & & \\
\hline
\end{tabular}


6336 SUB $\operatorname{Mpwr}(P(*)$, Row, SP, Wr $(*), G \$, X, Y, P \$, P, \operatorname{Res}) !-81 / 07 / 16)$ - - GFE 6339 DIM W(1:1,1:4)

6342 CALL $W(P(*)$, Row $, S p=1, S p=2,1, W r(*), W(*))$

6345 CALL Cmpy(Wr(Sp, 10), Wr $\left.\left(S_{p}, 11\right), W(1,1), W(1,2), A x, A y\right)$

6348 CALL Csub(Wr(Sp, 6), $\operatorname{Wr}(S p, 7), A x, A y, A x, A y)$

$6351 \mathrm{P}=\mathrm{Pinc}=\mathrm{W}(1,3) * \mathrm{Wr}(\mathrm{SP}, 12) *(\mathrm{Ax} * \mathrm{Ax}+\mathrm{Ay} * A y)$

6354 IF $(G \$=" R ")+(G \$=" P ")+(P \$=" N e t ")=0$ THEN Exit

6357 CALL $\operatorname{Wg}(\operatorname{Wr}(*), S p, 0, W(1,1), W(1,2), X, Y)$

6360 IF $P \$=" N e t "$ THEN $P=P *(1-X * X-Y * Y)$

6363 IF G\$="P" THEN CALL Polar $(X, Y, X, Y)$

6366 Exit: Res=W $(1,4)$

6369 SUBEND

S Mpwr(

Ax

$\begin{array}{lllll}6345 & 6348 & 6348 & 6351 & 6351\end{array}$

Ay

$\begin{array}{lllll}6345 & 6348 & 6348 & 6351 & 6351\end{array}$

Exit:

$6366 \quad 6354$

GS

$6336 \quad 6354 \quad 6354 \quad 6363$

$\mathbf{P}$

$\begin{array}{llll}6336 & 6351 \quad 6360 \quad 6360\end{array}$

PS

$6336 \quad 6354 \quad 6360$

$P($

$6336 \quad 6342$

Pinc

6351

Res

$6336 \quad 6366$

Row

$6336 \quad 6342$

$\mathrm{Sp}$

$6336 \quad 6342$

6351

6357

W(

6339

6342

6345

6345

6351

$\begin{array}{lll}6345 & 6348 \quad 6348\end{array}$

6366

Wr(

6336

$6342 \quad 6345$

6345

$6348 \quad 6348$

6351

$\mathrm{X}$

6357

6360

6360

$6363 \quad 6363$

$\mathrm{Y}$

6336

$\begin{array}{lllll}6357 & 6360 & 6360 & 6363 & 6363\end{array}$ 
6372 SUB Mtpt(P(*), Row, Stp,Wr(*), F\$,S(*), R(*)) !-(81/07/16) - -GFE

6375 MAT R-ZER

6378 DIM $W(1: 4,1: 8), C(1: 6), G(1: 4,1: 4)$

6381 CALL $W(P(*)$, Row $4,4, S t p, W r(*), W(*))$

6384 FOR I=1 TO 4

6387 FOR $\mathrm{SP}=1$ TO 2

$6390 \mathrm{~J}=(\mathrm{Sp}=1)+5 *(\mathrm{Sp}=2)$

$6393 \operatorname{CALL} W g(W r(*), S p, 0, W(I, J), W(I, J+1), G(I, 2 * S p-1), G(I, 2 * S p))$

$6396 R(S p)-R(S p)+W(I, 4 * S p)$

6399 NEXT SP

6402 NEXT I

6405 MAT R-R/(4)

6408 CALL Rtpt $(G(*), 1, C(*), R(3))$

6411 CALL Cmpy $(C(2), C(5), C(3), C(6), A x, A y)$

6414 CALL Csub(C(1),C(4), Ax, Ay, Ax, Ay)

6417 CALL Polar (Ax, Ay, R, T)

$6420 \mathrm{~T}=\mathrm{T} / 2-180 *(\mathrm{~T}>0) \quad !-180<($ Default value $)<0$

6423 IF $F \$=" P "$ THEN $P$

6426 CALL Rect(SQR(R), T, X, Y)

6429 CALL Ident( $S(1), C(2), S(2), C(5), S(5),-C(3), S(6),-C(6), S(3), X$,

6432 GOTO Exit $\mathrm{S}(4), \mathrm{Y})$

6435 P: CALL Polar(C(2), C(5), S(1), S(2))

6438 CALL Polar $(-C(3),-C(6), S(5), S(6))$

$6441 \mathrm{~S}(3)=\mathrm{SQR}(\mathrm{R})$

$6444 \mathrm{~S}(4)=\mathrm{T}$

6447 SUBEND

6450 Pset: ! PSET AT 3450

6460 RE-SAVE "ADP11",6460,11000 !850730

CODED TO UTILIZE DUAL SIX-PORT

S Mtpt(

Ax

$\begin{array}{llll}6411 & 6414 & 6414 & 6417\end{array}$

Ay

$\begin{array}{llll}6411 & 6414 & 6414 & 6417\end{array}$

C

$\begin{array}{llll}6378 & 6408 & 6411 & 6411\end{array}$

$\begin{array}{llll}6414 & 6429 & 6429 & 6429\end{array}$

$6411 \quad 6411 \quad 6414$

$6438 \quad 6438$

Exit:

$0 \quad 6432$

F\$

$6372 \quad 6423$

G(

$\begin{array}{llll}6378 & 6393 & 6393 & 6408\end{array}$

I

$\begin{array}{lllllll}6384 & 6393 & 6393 & 6393 & 6393 & 6396 & 6402\end{array}$

$\mathrm{J}$

$6390 \quad 6393 \quad 6393$

$P($

$6372 \quad 6381$

P :

64356423 


$\begin{array}{llllllll}\text { Pset: } & 6450 & & & & & & \\ \text { R } & 6417 & 6426 & 6441 & & & & \\ \text { R( } & 6372 & 6375 & 6396 & 6396 & 6405 & 6405 & 6408 \\ \text { Row } & 6372 & 6381 & & & & & \\ \text { S( } & 6372 & 6429 & 6429 & 6429 & 6429 & 6429 & 6429 \\ & 6435 & 6435 & 6438 & 6438 & 6441 & 6444 & \\ \text { Sp } & 6387 & 6390 & 6390 & 6393 & 6393 & 6393 & 6396 \\ \text { Stp } & 6396 & 6396 & 6399 & & & & \\ \text { T } & 6372 & 6381 & & & & & \\ \text { W( } & 6417 & 6420 & 6420 & 6420 & 6426 & 6444 & \\ \text { Wr( } & 6378 & 6381 & 6393 & 393 & 6396 & & \\ \text { X } & 6372 & 6381 & 6393 & & & & \\ \text { Y } & 6426 & 6429 & & & & & \\ & 6426 & 6429 & & & & & \end{array}$


6470 DEF FNAdp (Nfreqs, Repeats, M1, P1, M2, P2, Ca\$)

6480 ! CALC PARAMETERS FOR ADAPTOR FROM 6PORT MEAS JULY-AUG 1985

6490 OPTION BASE 1

6500 MsuŞ=":F8, 1"

6510 DIM Result(Nfreqs, 60), Freqs(Nfreqs), Id_content $[80]$,

$6521 \quad K=0$ S (Nfreqs, 34)

6570 ! LINPUT "ENTER FILE NAME FOR DATA WITHOUT ADAPTER",N1\$

6580 FIXED 0

6590 ! LINPUT "ENTER FILE NAME FOR DATA WITH ADAPTER

6620 N1\$="CRSTI"

$6630 \mathrm{~N} 2 \$="$ CRST3"

6640 CALL Redhed(N1\$,Msu\$, Date\$, Time\$, Typ\$,Nfreqs, Freqs (*),

6650 MASS STORAGE IS ":F8, 1 "

Repeats, Sp, Std_mnt, Id_content\$, Cnnct $\$$ )

6660 PRINTER IS 16

6670 PRINT "START OF FNADPT"

6680 Nfreq=N=Nfreqs

6690 PRINT

6700 PRINT

6710 Sport=1

6720 PRINTER IS 0

6721 PRINT

6724 PRINT "ADAPTER EVALUATION"

6725 PRINT "-................

6727 PRINT

6730 PRINT "FILES PROCESSED =";N1\$;" AND ";N2\$

6740 PRINT

$6750 \mathrm{~N} 1=\mathrm{INT}(\mathrm{N} 1)$

6760 N2=INT(N2)

6770 PRINTER IS 16

6780 FOR $P=1$ TO Nfreqs

6790 FOR Prt=1 TO 2

6800 PRINTER IS 16

6810 ON Prt GOTO 6820,6850

6820 CALL Redwrt(N1\$, ":F8,1"," ",Result(*), Nfreqs)

$6830 \quad$ Nfreq $=1$

6840 GOTO 6870

6850 CALL Redwrt(N2\$, ":F8,1", " ",Result(*), Nfreqs)

$6860 \quad$ NORMAL

6870 ON Sport GOTO 6880,6900

$6880 \mathrm{MC}=0$

6890 GOTO 6910

$6900 \mathrm{Mc}=12$

6910 A8-Result $(P, 6+M c)$

6920 A9=Result $(P, 7+M c)$

6930 B8 $=\operatorname{Result}(P, 8+M C)$

6940 B9-Result (P,9+MC)

$6950 \quad C 8=\operatorname{Result}(P, 10+\mathrm{MC})$

6960 C9=Result $(P, 11+M C)$

6980 GOTO 6990

$6990 \quad A 11$ (Prt) $=A 8$

7000 A22(Prt) $=$ A9 


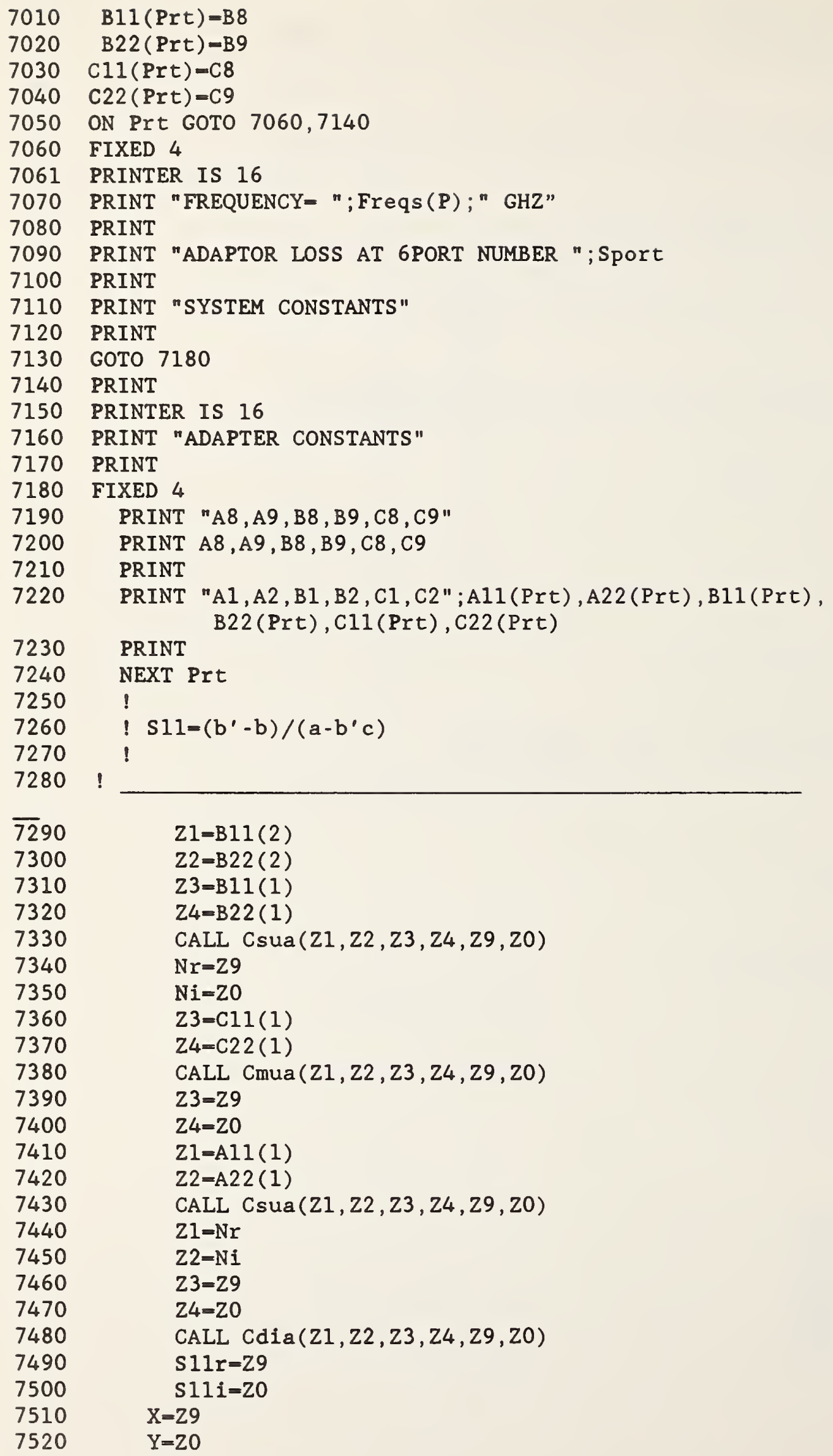




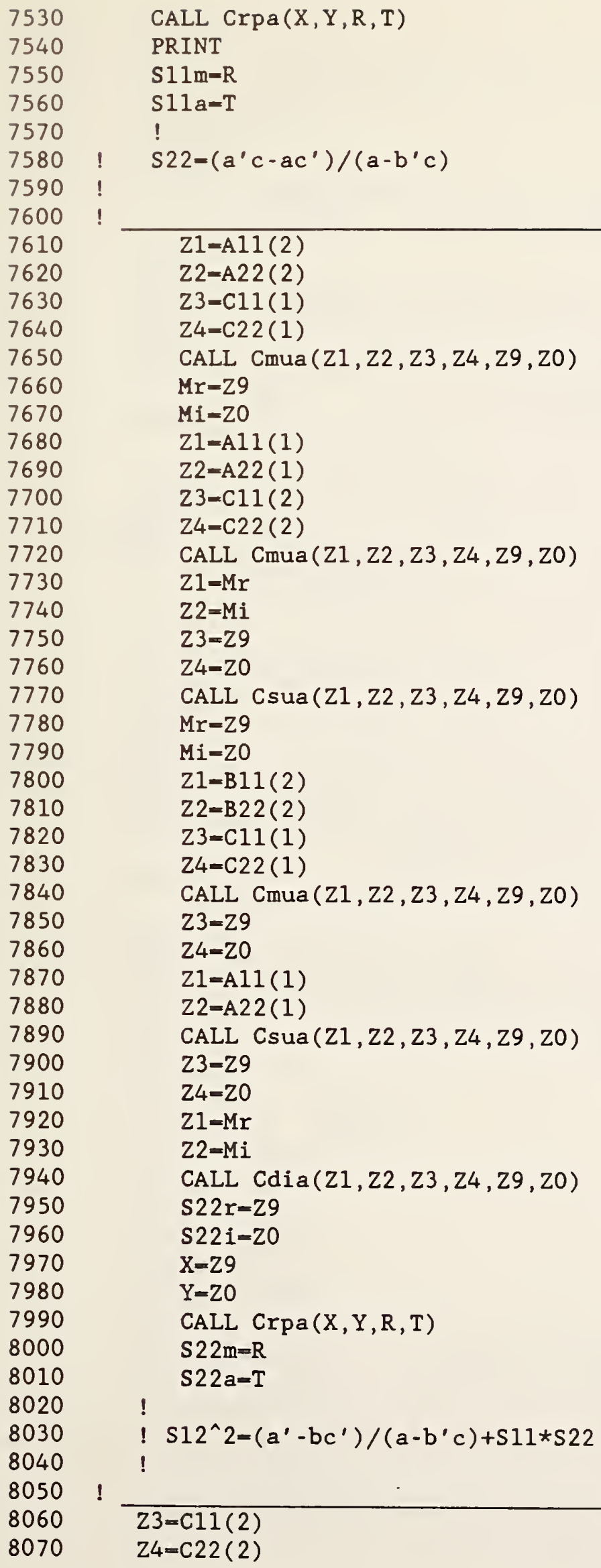




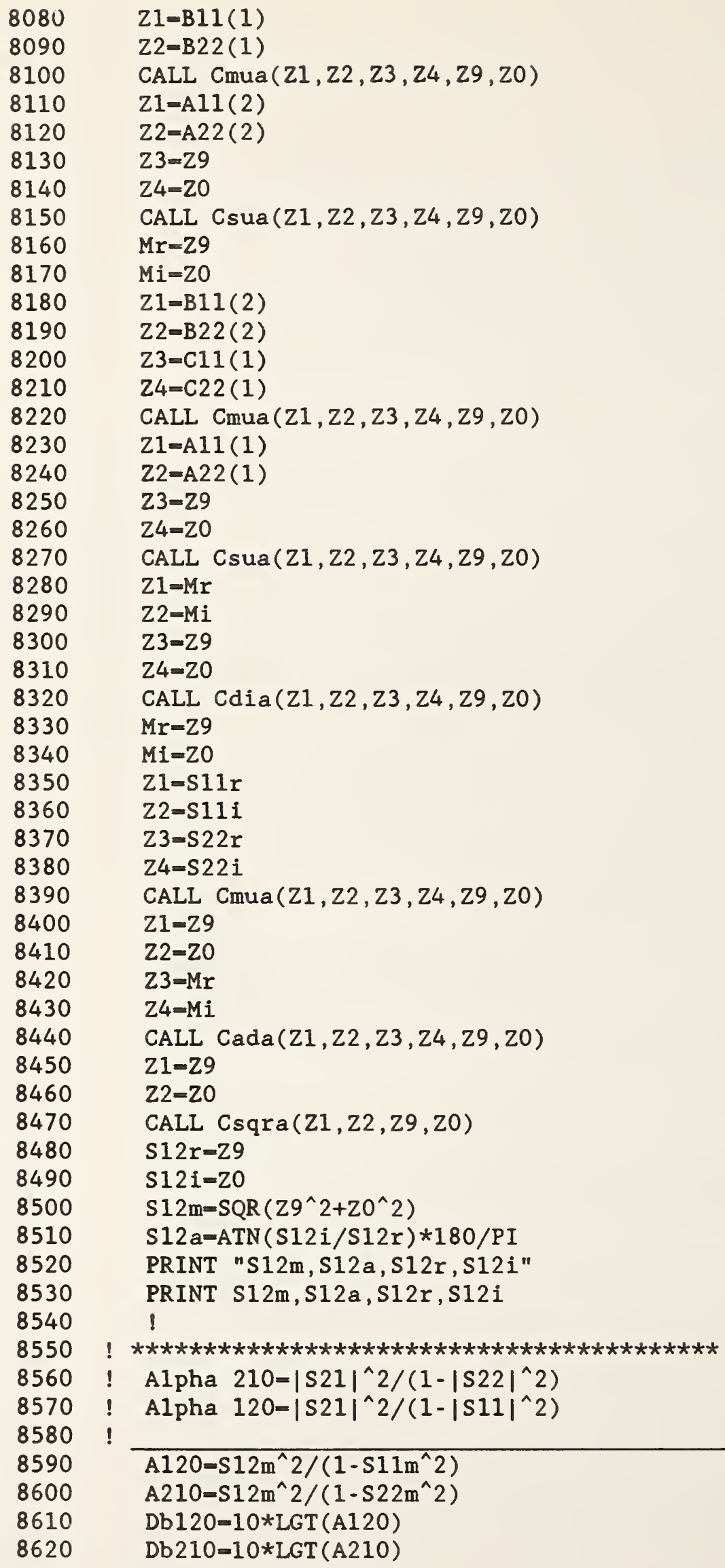




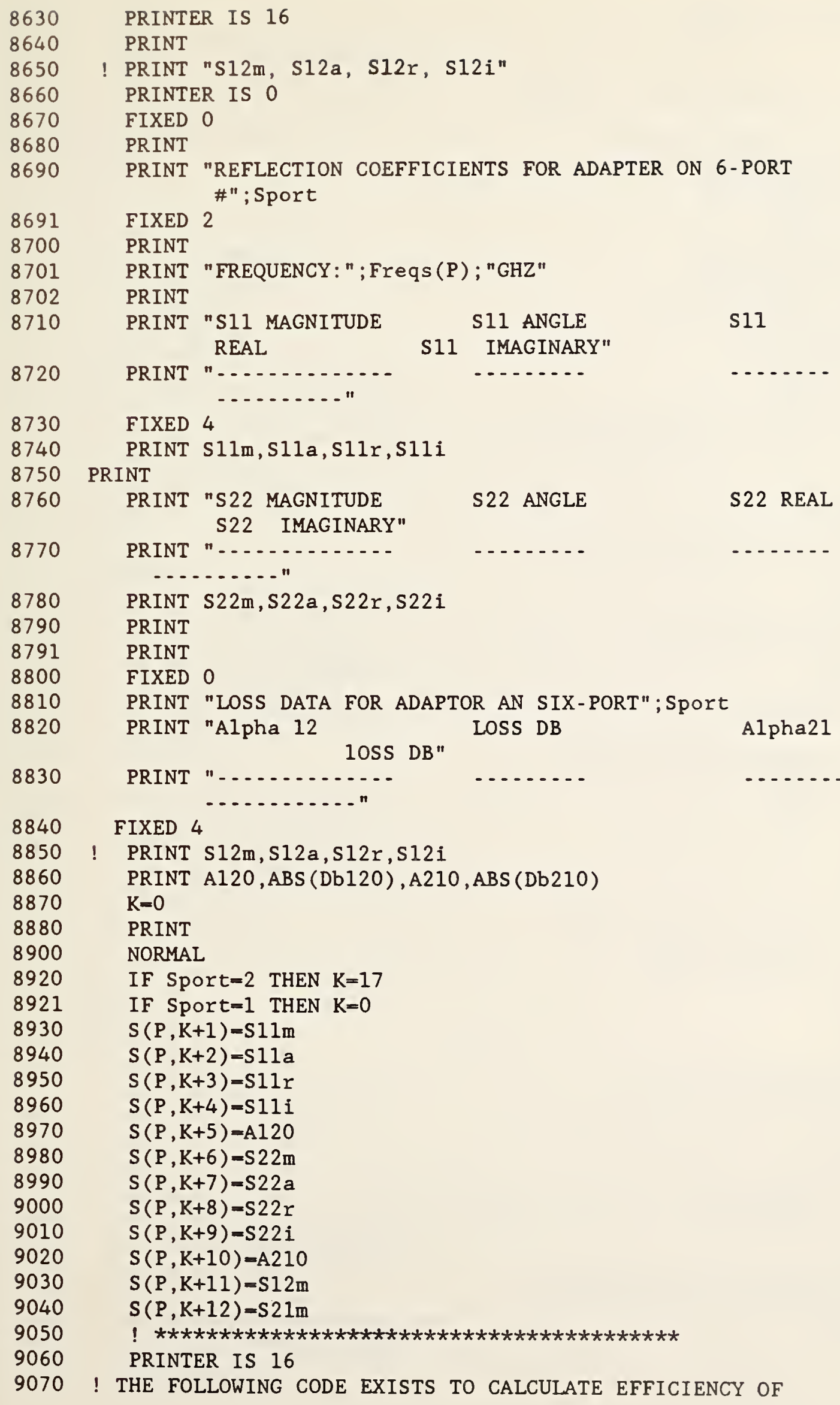




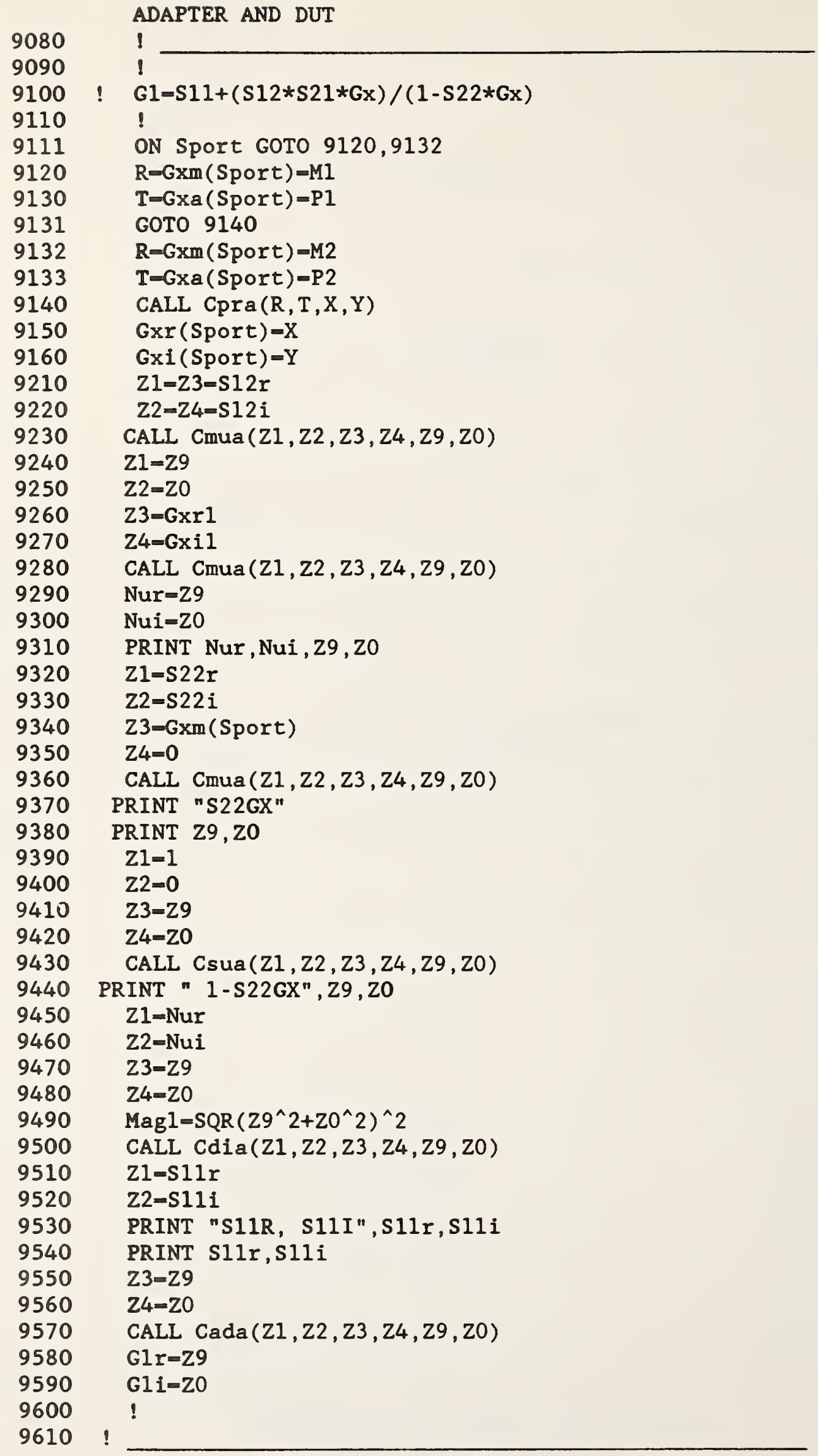




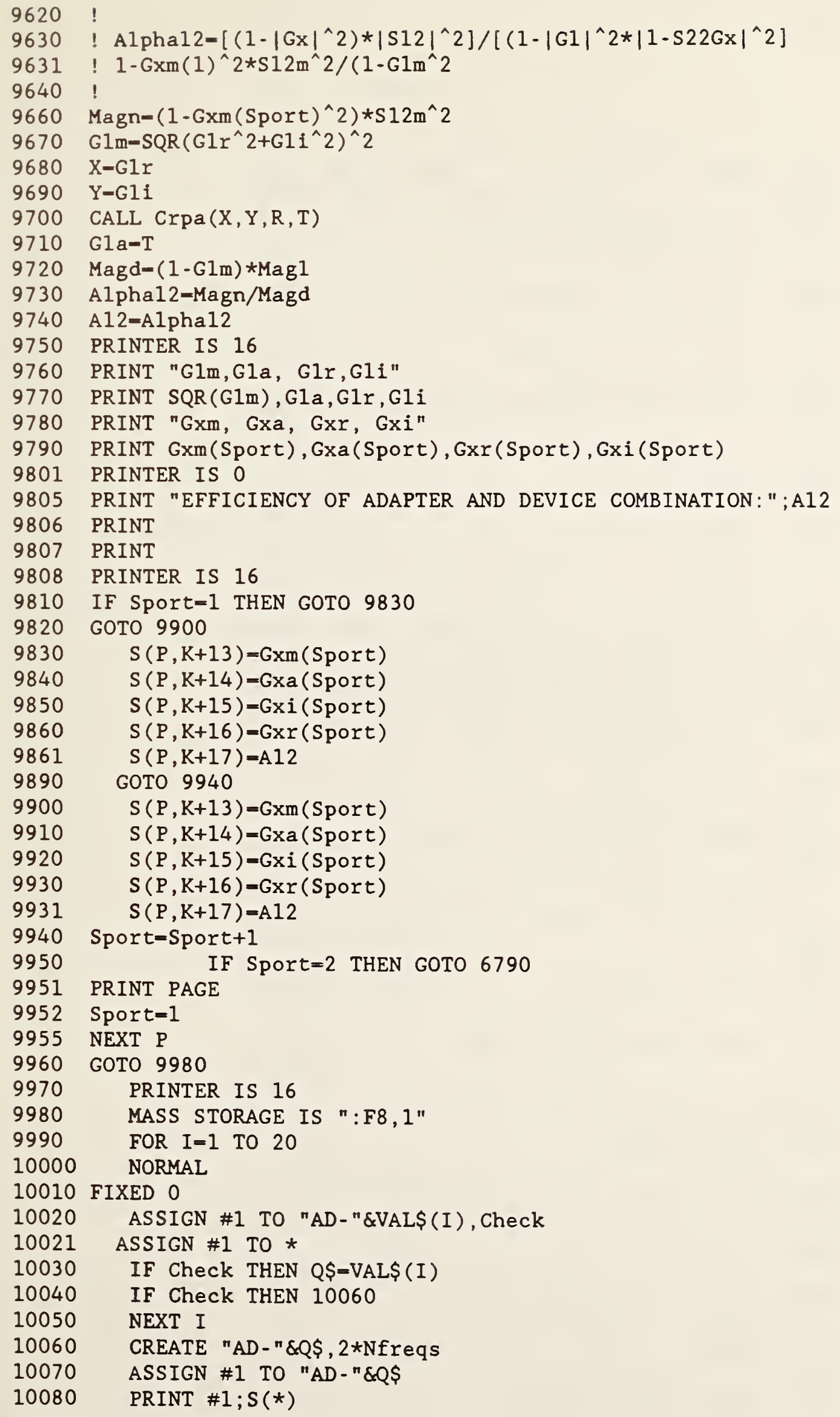


FNAdp (

\begin{tabular}{|c|c|c|c|c|c|c|c|}
\hline Al1( & $\begin{array}{l}6990 \\
8230\end{array}$ & 7220 & 7410 & 7610 & 7680 & 7870 & 8110 \\
\hline Al2 & 9740 & 9805 & 9861 & 9931 & & & \\
\hline A120 & 8590 & 8610 & 8860 & 8970 & & & \\
\hline A210 & 8600 & 8620 & 8860 & 9020 & & & \\
\hline A22( & $\begin{array}{l}7000 \\
8240\end{array}$ & 7220 & 7420 & 7620 & 7690 & 7880 & 8120 \\
\hline A8 & 6910 & 6990 & 7200 & & & & \\
\hline A9 & 6920 & 7000 & 7200 & & & & \\
\hline Alphal 2 & 9730 & 9740 & & & & & \\
\hline B11( & 7010 & 7220 & 7290 & 7310 & 7800 & 8080 & 8180 \\
\hline B22( & 7020 & 7220 & 7300 & 7320 & 7810 & 8090 & 8190 \\
\hline B8 & 6930 & 7010 & 7200 & & & & \\
\hline B9 & 6940 & 7020 & 7200 & & & & \\
\hline C11( & $\begin{array}{l}7030 \\
8200\end{array}$ & 7220 & 7360 & 7630 & 7700 & 7820 & 8060 \\
\hline $\mathrm{C} 22($ & $\begin{array}{l}040 \\
8210\end{array}$ & 7220 & 7370 & 7640 & 7710 & 7830 & 8070 \\
\hline $\mathrm{C} 8$ & 6950 & 7030 & 7200 & & & & \\
\hline C9 & 6960 & 7040 & 7200 & & & & \\
\hline Cas & 6470 & & & & & & \\
\hline Check & 10020 & 10030 & 10040 & & & & \\
\hline Cnnct\$ & 6640 & & & & & & \\
\hline Date\$ & 6640 & & & & & & \\
\hline $\mathrm{Db} 120$ & 8610 & 8860 & & & & & \\
\hline $\mathrm{Db} 210$ & 8620 & 8860 & & & & & \\
\hline Freqs ( & 6510 & 6640 & 7070 & 8701 & & & \\
\hline
\end{tabular}




\begin{tabular}{|c|c|c|c|c|c|c|c|}
\hline Gla & 9710 & 9770 & & & & & \\
\hline G1I & 9590 & 9670 & 9690 & 9770 & & & \\
\hline Glm & 9670 & 9720 & 9770 & & & & \\
\hline Glr & 9580 & 9670 & 9680 & 9770 & & & \\
\hline Gxal & 9130 & 9133 & 9790 & 9840 & 9910 & & \\
\hline Gxic & 9160 & 9790 & 9850 & 9920 & & & \\
\hline $\mathrm{Gx} 11$ & 9270 & & & & & & \\
\hline Gxm ( & 9120 & 9132 & 9340 & 9660 & 9790 & 9830 & 9900 \\
\hline Gxr ( & 9150 & 9790 & 9860 & 9930 & & & \\
\hline Gxr 1 & 9260 & & & & & & \\
\hline I & 9990 & 10020 & 10030 & 10050 & & & \\
\hline Id_content\$ & 6510 & 6640 & & & & & \\
\hline $\mathrm{K}$ & $\begin{array}{l}6521 \\
8960 \\
9030 \\
9900\end{array}$ & $\begin{array}{l}8870 \\
8970 \\
9040 \\
9910\end{array}$ & $\begin{array}{l}8920 \\
8980 \\
9830 \\
9920\end{array}$ & $\begin{array}{l}8921 \\
8990 \\
9840 \\
9930\end{array}$ & $\begin{array}{l}8930 \\
9000 \\
9850 \\
9931\end{array}$ & $\begin{array}{l}8940 \\
9010 \\
9860\end{array}$ & $\begin{array}{l}8950 \\
9020 \\
9861\end{array}$ \\
\hline M1 & 6470 & 9120 & & & & & \\
\hline M2 & 6470 & 9132 & & & & & \\
\hline Mag1 & 9490 & 9720 & & & & & \\
\hline Magd & 9720 & 9730 & & & & & \\
\hline Magn & 9660 & 9730 & & & & & \\
\hline Mc & $\begin{array}{l}6880 \\
6960\end{array}$ & 6900 & 6910 & 6920 & 6930 & 6940 & 6950 \\
\hline Mi & $\begin{array}{l}7670 \\
8430\end{array}$ & 7740 & 7790 & 7930 & 8170 & 8290 & 8340 \\
\hline $\mathrm{Mr}$ & $\begin{array}{l}7660 \\
8420\end{array}$ & 7730 & 7780 & 7920 & 8160 & 8280 & 8330 \\
\hline MsuS & 6500 & 6640 & & & & & \\
\hline $\mathrm{N}$ & 6680 & 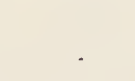 & & & & & \\
\hline $\mathrm{N} 1$ & 6750 & 6750 & & & & & \\
\hline
\end{tabular}




\begin{tabular}{|c|c|c|c|c|c|c|c|}
\hline N1\$ & 6620 & 6640 & 6730 & 6820 & & & \\
\hline N2 & 6760 & 6760 & & & & & \\
\hline $\mathrm{N} 2 \$$ & 6630 & 6730 & 6850 & & & & \\
\hline Nfreq & 6680 & 6830 & & & & & \\
\hline Nfreqs & $\begin{array}{l}6470 \\
6820\end{array}$ & $\begin{array}{l}6510 \\
6850\end{array}$ & $\begin{array}{r}6510 \\
10060\end{array}$ & 6510 & 6640 & 6680 & 6780 \\
\hline $\mathrm{Ni}$ & 7350 & 7450 & & & & & \\
\hline $\mathrm{Nr}$ & 7340 & 7440 & & & & & \\
\hline Nui & 9300 & 9310 & 9460 & & & & \\
\hline Nur & 9290 & 9310 & 9450 & & & & \\
\hline 0 & 10110 & & & & & & \\
\hline $\mathbf{P}$ & $\begin{array}{l}6780 \\
7070 \\
8980 \\
9830 \\
9920\end{array}$ & $\begin{array}{l}6910 \\
8701 \\
8990 \\
9840 \\
9930\end{array}$ & $\begin{array}{l}6920 \\
8930 \\
9000 \\
9850 \\
9931\end{array}$ & $\begin{array}{l}6930 \\
8940 \\
9010 \\
9860 \\
9955\end{array}$ & $\begin{array}{l}6940 \\
8950 \\
9020 \\
9861\end{array}$ & $\begin{array}{l}6950 \\
8960 \\
9030 \\
9900\end{array}$ & $\begin{array}{l}6960 \\
8970 \\
9040 \\
9910\end{array}$ \\
\hline P1 & 6470 & 9130 & & & & & \\
\hline P2 & 6470 & 9133 & & & & & \\
\hline Prt & $\begin{array}{l}6790 \\
7040 \\
7220\end{array}$ & $\begin{array}{l}6810 \\
7050 \\
7240\end{array}$ & $\begin{array}{l}6990 \\
7220\end{array}$ & $\begin{array}{l}7000 \\
7220\end{array}$ & $\begin{array}{l}7010 \\
7220\end{array}$ & $\begin{array}{l}7020 \\
7220\end{array}$ & $\begin{array}{l}7030 \\
7220\end{array}$ \\
\hline Q\$ & 0 & 10030 & 10030 & 10060 & 10070 & & \\
\hline $\mathbf{R}$ & $\begin{array}{l}7530 \\
9700\end{array}$ & 7550 & 7990 & 8000 & 9120 & 9132 & 9140 \\
\hline Repeats & 6470 & 6640 & & & & & \\
\hline Result( & $\begin{array}{l}6510 \\
6950\end{array}$ & $\begin{array}{l}6820 \\
6960\end{array}$ & 6850 & 6910 & 6920 & 6930 & 6940 \\
\hline S( & $\begin{array}{l}6510 \\
8990 \\
9840 \\
9930\end{array}$ & $\begin{array}{l}8930 \\
9000 \\
9850 \\
9931\end{array}$ & $\begin{array}{r}8940 \\
9010 \\
9860 \\
10080\end{array}$ & $\begin{array}{l}8950 \\
9020 \\
9861\end{array}$ & $\begin{array}{l}8960 \\
9030 \\
9900\end{array}$ & $\begin{array}{l}8970 \\
9040 \\
9910\end{array}$ & $\begin{array}{l}8980 \\
9830 \\
9920\end{array}$ \\
\hline s11a & 7560 & 8740 & 8940 & & & & \\
\hline s11i & 7500 & 8360 & 8740 & 8960 & 9520 & 9530 & 9540 \\
\hline S11m & 7550 & 8590 & 8740 & 8930 & & & \\
\hline
\end{tabular}




\begin{tabular}{|c|c|c|c|c|c|c|c|}
\hline $\operatorname{silr}$ & 7490 & 8350 & 8740 & 8950 & 9510 & 9530 & 9540 \\
\hline$S 12 a$ & 8510 & 8530 & & & & & \\
\hline$S 12 i$ & 8490 & 8510 & 8530 & 9220 & & & \\
\hline $\mathrm{S} 12 \mathrm{~m}$ & 8500 & 8530 & 8590 & 8600 & 9030 & 9660 & \\
\hline$S 12 r$ & 8480 & 8510 & 8530 & 9210 & & & \\
\hline S21m & 9040 & & & & & & \\
\hline s22a & 8010 & 8780 & 8990 & & & & \\
\hline$S 22 i$ & 7960 & 8380 & 8780 & 9010 & 9330 & & \\
\hline $\mathrm{S} 22 \mathrm{~m}$ & 8000 & 8600 & 8780 & 8980 & & & \\
\hline$S 22 r$ & 7950 & 8370 & 8780 & 9000 & 9320 & & \\
\hline Sp & 6640 & & & & & & \\
\hline Sport & $\begin{array}{l}6710 \\
9111 \\
9340 \\
9830 \\
9930\end{array}$ & $\begin{array}{l}6870 \\
9120 \\
9660 \\
9840 \\
9940\end{array}$ & $\begin{array}{l}7090 \\
9130 \\
9790 \\
9850 \\
9940\end{array}$ & $\begin{array}{l}8690 \\
9132 \\
9790 \\
9860 \\
9950\end{array}$ & $\begin{array}{l}8810 \\
9133 \\
9790 \\
9900 \\
9952\end{array}$ & $\begin{array}{l}8920 \\
9150 \\
9790 \\
9910\end{array}$ & $\begin{array}{l}8921 \\
9160 \\
9810 \\
9920\end{array}$ \\
\hline Std_mnt & 6640 & & & & & & \\
\hline $\mathrm{T}$ & $\begin{array}{l}7530 \\
9700\end{array}$ & $\begin{array}{l}7560 \\
9710\end{array}$ & 7990 & 8010 & 9130 & 9133 & 9140 \\
\hline Time\$ & 6640 & & & & & & \\
\hline Typ\$ & 6640 & & & & & & \\
\hline $\mathrm{X}$ & $\begin{array}{l}7510 \\
9700\end{array}$ & 7530 & 7970 & 7990 & 9140 & 9150 & 9680 \\
\hline $\mathrm{Y}$ & $\begin{array}{l}7520 \\
9700\end{array}$ & 7530 & 7980 & 7990 & 9140 & 9160 & 9690 \\
\hline 20 & $\begin{array}{l}7330 \\
7500 \\
7790 \\
7980 \\
8270 \\
8460 \\
9300 \\
9480\end{array}$ & $\begin{array}{l}7350 \\
7520 \\
7840 \\
8100 \\
8310 \\
8470 \\
9310 \\
9490\end{array}$ & $\begin{array}{l}7380 \\
7650 \\
7860 \\
8140 \\
8320 \\
8490 \\
9360 \\
9500\end{array}$ & $\begin{array}{l}7400 \\
7670 \\
7890 \\
8150 \\
8340 \\
8500 \\
9380 \\
9560\end{array}$ & $\begin{array}{l}7430 \\
7720 \\
7910 \\
8170 \\
8390 \\
9230 \\
9420 \\
9570\end{array}$ & $\begin{array}{l}7470 \\
7760 \\
7940 \\
8220 \\
8410 \\
9250 \\
9430 \\
9590\end{array}$ & $\begin{array}{l}7480 \\
7770 \\
7960 \\
8260 \\
8440 \\
9280 \\
9440\end{array}$ \\
\hline 21 & $\begin{array}{l}7290 \\
7610\end{array}$ & $\begin{array}{l}7330 \\
7650\end{array}$ & $\begin{array}{l}7380 \\
7680\end{array}$ & $\begin{array}{l}7410 \\
7720\end{array}$ & $\begin{array}{l}7430 \\
7730\end{array}$ & $\begin{array}{l}7440 \\
7770\end{array}$ & $\begin{array}{l}7480 \\
7800\end{array}$ \\
\hline
\end{tabular}


Z2

$\begin{array}{lllllll}7840 & 7870 & 7890 & 7920 & 7940 & 8080 & 8100 \\ 8110 & 8150 & 8180 & 8220 & 8230 & 8270 & 8280 \\ 8320 & 8350 & 8390 & 8400 & 8440 & 8450 & 8470 \\ 9210 & 9230 & 9240 & 9280 & 9320 & 9360 & 9390 \\ 9430 & 9450 & 9500 & 9510 & 9570 & & \\ 7300 & 7330 & 7380 & 7420 & 7430 & 7450 & 7480 \\ 7620 & 7650 & 7690 & 7720 & 7740 & 7770 & 7810 \\ 7840 & 7880 & 7890 & 7930 & 7940 & 8090 & 8100 \\ 8120 & 8150 & 8190 & 8220 & 8240 & 8270 & 8290 \\ 8320 & 8360 & 8390 & 8410 & 8440 & 8460 & 8470 \\ 9220 & 9230 & 9250 & 9280 & 9330 & 9360 & 9400 \\ 9430 & 9460 & 9500 & 9520 & 9570 & & \\ 7310 & 7330 & 7360 & 7380 & 7390 & 7430 & 7460 \\ 7480 & 7630 & 7650 & 7700 & 7720 & 7750 & 7770 \\ 7820 & 7840 & 7850 & 7890 & 7900 & 7940 & 8060 \\ 8100 & 8130 & 8150 & 8200 & 8220 & 8250 & 8270 \\ 8300 & 8320 & 8370 & 8390 & 8420 & 8440 & 9210 \\ 9230 & 9260 & 9280 & 9340 & 9360 & 9410 & 9430 \\ 9470 & 9500 & 9550 & 9570 & & & \\ 7320 & 7330 & 7370 & 7380 & 7400 & 7430 & 7470 \\ 7480 & 7640 & 7650 & 7710 & 7720 & 7760 & 7770 \\ 7830 & 7840 & 7860 & 7890 & 7910 & 7940 & 8070 \\ 8100 & 8140 & 8150 & 8210 & 8220 & 8260 & 8270 \\ 8310 & 8320 & 8380 & 8390 & 8430 & 8440 & 9220 \\ 9230 & 9270 & 9280 & 9350 & 9360 & 9420 & 9430 \\ 9480 & 9500 & 9560 & 9570 & & & \\ 7330 & 7340 & 7380 & 7390 & 7430 & 7460 & 7480 \\ 7490 & 7510 & 7650 & 7660 & 7720 & 7750 & 7770 \\ 7780 & 7840 & 7850 & 7890 & 7900 & 7940 & 7950 \\ 7970 & 8100 & 8130 & 8150 & 8160 & 8220 & 8250 \\ 8270 & 8300 & 8320 & 8330 & 8390 & 8400 & 8440 \\ 8450 & 8470 & 8480 & 8500 & 9230 & 9240 & 9280 \\ 9290 & 9310 & 9360 & 9380 & 9410 & 9430 & 9440 \\ 9470 & 9490 & 9500 & 9550 & 9570 & 9580 & \\ & & & & & & \end{array}$


10120 SUB Cmua $(Z 1, Z 2, Z 3, Z 4, Z 9, Z 0)$

$1013029-\mathrm{Z} 1 * \mathrm{Z} 3-\mathrm{Z} 2 * \mathrm{Z} 4$

$10140 \quad 20-21 * 24+22 * 23$

10150 SUBEXIT

10160 SUBEND

S Cmual

20

21

22

23

24

29
1012010140

$10120 \quad 10130 \quad 10140$

$1012010130 \quad 10140$

$10120 \quad 10130 \quad 10140$

$10120 \quad 10130 \quad 10140$

$10120 \quad 10130$ 
10170 SUB Cdia(Z1,Z2,Z3,Z4,Z9,Z0)

$10180 \mathrm{Z} 6=\mathrm{Z} 3 * \mathrm{Z} 3+\mathrm{Z} 4 * \mathrm{Z} 4$

$10190 \mathrm{Z9}=(\mathrm{Z} 1 * \mathrm{Z} 3+\mathrm{Z} 2 * \mathrm{Z} 4) / \mathrm{Z} 6$

$10200 \mathrm{ZO}=(\mathrm{Z} 2 * \mathrm{Z} 3-\mathrm{Z} 1 * \mathrm{Z} 4) / \mathrm{Z} 6$

10210 SUBEXIT

10220 SUBEND

S Cdial

20

21

22

23

24

26

29
1017010200

$1017010190 \quad 10200$

$10170 \quad 10190 \quad 10200$

$\begin{array}{lllll}10170 & 10180 & 10180 & 10190 & 10200\end{array}$

$\begin{array}{lllll}10170 & 10180 & 10180 & 10190 & 10200\end{array}$

$1018010190 \quad 10200$

$10170 \quad 10190$ 
10230 SUB Cinva $(\mathrm{Z} 1, \mathrm{Z2}, \mathrm{Z9}, \mathrm{Z0})$

$10240 \mathrm{Z} 6-\mathrm{Z} 1 * \mathrm{Z} 1+\mathrm{Z} 2 * \mathrm{Z} 2$

$10250 \quad \mathrm{Z9}-\mathrm{Z} 1 / \mathrm{Z} 6$

$10260 \mathrm{ZO}=-\mathrm{Z} 2 / \mathrm{Z} 6$

10270 SUBEXIT

10280 SUBEND

S Cinval

20

21

22

26

29
1023010260

$\begin{array}{llll}10230 & 10240 \quad 10240 \quad 10250\end{array}$

$\begin{array}{llll}10230 & 10240 \quad 10240 \quad 10260\end{array}$

$10240 \quad 10250 \quad 10260$

$10230 \quad 10250$ 
10290 SUB Csqa $(\mathrm{Z} 1, \mathrm{Z} 2, \mathrm{Z9}, \mathrm{Z} 0)$

$10300 \mathrm{Z9}=\mathrm{Z} 1 * \mathrm{Z} 1-\mathrm{Z} 2 * \mathrm{Z} 2$

$10310 \quad \mathrm{Z} 0=2 * \mathrm{Z} 1 * \mathrm{Z} 2$

10320 SUBEXIT

10330 SUBEND

\section{S Csqal}

20

21

22

29
$10290 \quad 10310$

$\begin{array}{llll}10290 & 10300 \quad 10300 \quad 10310\end{array}$

$\begin{array}{llll}10290 & 10300 \quad 10300 \quad 10310\end{array}$

$10290 \quad 10300$ 
10340 SUB Cada $(Z 1, Z 2, Z 3, Z 4, Z 9,20)$

$10350 \quad 29-21+Z 3$

$10360 \mathrm{ZO}=\mathrm{Z2}+\mathrm{Z} 4$

10370 SUBEXIT

10380 SUBEND

S Cadal

20

$10340 \quad 10360$

21

$10340 \quad 10350$

22

$10340 \quad 10360$

23

$10340 \quad 10350$

24

$10340 \quad 10360$

29

$10340 \quad 10350$ 
10390 SUB Csua $(21,22, Z 3,24, Z 9, Z 0)$

10400 Z9-Z1-Z3

$10410 \quad \mathrm{Z} 0=\mathrm{Z} 2-\mathrm{Z} 4$

10420 SUBEXIT

10430 SUBEND

\section{S Csual}

$\begin{array}{lll}\text { Z0 } & 10390 & 10410 \\ Z 1 & 10390 & 10400 \\ Z 2 & 10390 & 10410 \\ Z 3 & 10390 & 10400 \\ Z 4 & 10390 & 10410 \\ 29 & 10390 & 10400\end{array}$


10440 SUB Caba $(21,22, Z 9)$

$1045029-\mathrm{SQR}(21 * 21+\mathrm{Z} 2 * \mathrm{Z2})$

10460 SUBEXIT

10470 SUBEND

S Cabal

21

22

29
$10440 \quad 10450 \quad 10450$

$10440 \quad 10450 \quad 10450$

$10440 \quad 10450$ 
10480 SUB Csqra $(Z 1, Z 2, Z 9,20)$

$10490 \mathrm{X}=\mathrm{Z1}$

$10500 \quad \mathrm{Y}=\mathrm{Z2}$

10510 CALL Crpa(X,Y,R,T)

$10520 \mathrm{R}=\mathrm{SQR}(\mathrm{R})$

$10530 \mathrm{~T}=\mathrm{T} / 2$

$10540 \mathrm{Z9}=\mathrm{R} * \cos (\mathrm{PI} * \mathrm{~T} / 180)$

$10550 \mathrm{ZO}=\mathrm{R} * \mathrm{SIN}(\mathrm{PI} * \mathrm{~T} / 180)$

10560 GOTO 10570

10570 SUBEXIT

10580 SUBEND

S Csqral

$\begin{array}{llllll}\mathrm{R} & 10510 & 10520 & 10520 & 10540 & 10550 \\ \mathrm{~T} & 10510 & 10530 & 10530 & 10540 & 10550 \\ \mathrm{X} & 10490 & 10510 & & & \\ \mathrm{Y} & 10500 & 10510 & & & \\ \mathrm{Z} 0 & 10480 & 10550 & & \\ \mathrm{Z1} & 10480 & 10490 & & \\ \mathrm{Z2} & 10480 & 10500 & & \\ \mathrm{Z9} & 10480 & 10540 & & \end{array}$


10590 SUB Crpa $(X, Y, R, T)$

$10600 \mathrm{R}=\mathrm{SQR}(\mathrm{X} * \mathrm{X}+\mathrm{Y} * \mathrm{Y})$

10610 IF $X=0$ THEN GOTO 10640

$10620 \mathrm{~T}-\mathrm{ATN}(\mathrm{Y} / \mathrm{X})$

10630 GOTO 10650

$10640 \mathrm{~T}=\mathrm{PI} \star \mathrm{SGN}(\mathrm{Y}) / 2$

$10650 \mathrm{~T}-\mathrm{T} * 180 / \mathrm{PI}$

10660 IF $\mathrm{X}>-0$ THEN 10710

10670 IF $\mathrm{Y}>0$ THEN 10700

$10680 \mathrm{~T}-\mathrm{T}-180$

10690 GOTO 10710

$10700 \mathrm{~T}-\mathrm{T}+180$

10710 SUBEXIT

10720 SUBEND

S Crpal

R

1059010600

$\mathrm{T}$

$\begin{array}{lllllll}10590 & 10620 & 10640 & 10650 & 10650 & 10680 & 10680\end{array}$

1070010700

$\begin{array}{llllll}10590 & 10600 & 10600 & 10610 & 10620 & 10660\end{array}$

$\begin{array}{llllll}10590 & 10600 & 10600 & 10620 & 10640 & 10670\end{array}$ 
10730 SUB Cmuada(Z1,Z2,Z3, Z4,Z5, Z6, Z7, Z8, Z9, Z0)

$10740 \quad \mathrm{Z} 9=\mathrm{Z} 1 * \mathrm{Z} 3-\mathrm{Z} 2 * \mathrm{Z} 4+\mathrm{Z} 5 * \mathrm{Z} 7-\mathrm{Z} 6 * \mathrm{Z} 8$

$10750 \mathrm{ZO}=\mathrm{Z} 1 * \mathrm{Z} 4+\mathrm{Z} 2 * \mathrm{Z} 3+\mathrm{Z} 5 * \mathrm{Z} 8+\mathrm{Z} 6 * \mathrm{Z} 7$

10760 SUBEXIT

10770 SUBEND

S Cmuadal

$\mathrm{ZO}$

Z1

22

23

$\mathrm{Z4}$

$\mathbf{2 5}$

26

27

$\mathrm{Z} 8$

Z9
$10730 \quad 10750$

$10730 \quad 10740 \quad 10750$

$10730 \quad 10740 \quad 10750$

$10730 \quad 10740 \quad 10750$

$10730 \quad 10740 \quad 10750$

$10730 \quad 10740 \quad 10750$

$10730 \quad 10740 \quad 10750$

$10730 \quad 10740 \quad 10750$

$10730 \quad 10740 \quad 10750$

$10730 \quad 10740$ 
10780 SUB Cpra(R, T,X,Y)

$10790 \mathrm{X}-\mathrm{R} \star \operatorname{COS}(\mathrm{PI} \star \mathrm{T} / 180)$

$10800 \mathrm{Y}-\mathrm{R} \star \mathrm{SIN}(\mathrm{PI} * \mathrm{~T} / 180)$

10810 SUBEXIT

10820 SUBEND

S Cpral

$\begin{array}{llll}R & 10780 & 10790 & 10800 \\ \text { T } & 10780 & 10790 & 10800 \\ \text { X } & 10780 & 10790 & \\ \text { Y } & 10780 & 10800 & \end{array}$


10830 SUB DOC

10840 LINPUT "Today'S Date--AS 850729" "Doda\$

10850 PRINTER IS 0

10860 PRINT PAGE

10870 PRINT "CHANGES LISTED ARE AS OF ";Doda\$

10880 PRINT "CHANGE 1. RENAMED TO TST1-FINAL NAME TO BE ADPTR5"

10890 PRINT "CHANGE 2. CHANGED DATE IN FNDtgs\$ FROM 84 TO 85"

10900 PRINT "CHANGE 3. RENAMED SUB Pset TO SUB psetdel (LINE 3456)"

10910 PRINT "CHANGE 4. CHANGED SUB Synth TO CALL SUB Synth_900

(LINES 2830 TO"

10920 PRINT " $\quad$ 2931)."

10930 PRINT "CHANGE 5. INSERTED SUB Synth_900 AT LINE 8000 AND SUB"

10940 PRINT" Pset AT 8140 AND SÜB Genlevel AT 8480"

10950 PRINT "CHANGE 6. ADDED Cal=1 AT LINE 31"

10960 PRINT "CHANGE 7. ENTER INPUT STATEMENT FOR Nfreqs

WHEN ADAPTR CALC ONLY IS

m

10970 PRINT "CHANGE 8. CHANGED TyP LOGIC TO INCLUDE TYP>5 AT LINE 75"

10980 PRINT "CHANGE 9. CHANGE TyP LOGIC AT LINE 76"

10990 PRINT "CHANGE 10. CHANGED OR ADDED LOGIC IN STATEMENTS 78 - 79 "

11000 PRINT "CHANGE 11. CHANGED OR ADDED Typ LOGIC IN LINES $115-116 "$

11010 PRINT "CHANGE 12. CHANGED OR ADDED LOGIC LINES 119 AND $121 "$

11020 PRINT "CHANGE 13. ADDED STATEMENT 164 AND 180"

11030 PRINT "CHANGE 14. CHANGED OR ADDED STATEMENTS AT LINES 224, $225,226,235, "$

11040 PRINT " 236,237,238, AND 239"

11050 PRINT "CHANGE 15. CHANGED FORMAT LINE 261"

11060 PRINT "CHANGE 16. ADDED LINE 275

11070 PRINT "CHANGE 17. ADDED VARIABLE Proc TO CALLING STATEMENT IN LINES

11080 PRINT " 282,315,AND 333."

11090 PRINT "CHANGE 18. ADDED OR CHANGED LOGIC IN LINES 367-371."

11100 PRINT "CHANGE 19. CHANGED FORMAT STATEMENT IN LINE 465/"

11110 PRINT "CHANGE 20. ADDED Cal TO SUB Caldat IN LINE 1077"

11120 PRINT "CHANGE 21. DEFINED Cal\$ IN LINE 1093"

11130 PRINT "CHANGE 22. ADDED OR CHANGED LOGIC IN LINES 1265-1272."

11140 PRINT "CHANGE 23. ADDED(\&)CaS TO STRING IN LINES 1299 AND 1302"

11150 PRINT "CHANGE 24. ADDED(\&)Ca\$ TO STRING IN LINES 1323 AND 1326"

11160 PRINT "CHANGE 25. ADDED Cal TO SUB Calres CALL AND SUB Caldat CALLS "

11170 PRINT " IN LINES 168, 171,1077, AND 3612"

11180 PRINT "CHANGE 26. DIMENSIONED MATRIX Rd(Nfreqs, 2, 12) IN LINE n

11190 PRINT "

11200 PRINT "CHANGE 27. CHANGED VARIABLE LIST IN SUB Coeff--ADDED Cal AND $\operatorname{Rd}(*) "$

11210 PRINT " -DELETED Omitf(*) IN LINES 3727 AND 3795 "

11220 PRINT "CHANGE 28. ADDED LINPUT STATEMENT AT LINE 3625."

11230 PRINT "CHANGE 29. ADDED \&CaS TO FILE NAMES IN LINES 3639 AND 3640"

11240 PRINT "CHANGE 30. ADDED LINES 3643 AND 3644." 
11250 PRINT "CHANGE 31. ADDED DO LOOP FROM 3648 TO 3658."

11260 PRINT "CHANGE 32. ADDED LINES 3767 AND 3772."

11270 PRINT "CHANGE 33. ADDED ADAPTER CALC SUB PRORAM FNAdpt AT LINE 9000."

11280 SUBEXIT

11290 SUBEND

S Doc

Doda\$

$10840 \quad 10870$ 
11300 SUB Gamma(Nfreqs, Repeats, Ca\$)

11310 OPTION BASE 1

11320 DIM Power(Nfreqs, Repeats, 16, 10), Po (1, 8), Result(Nfreqs, 60), Wrspt $(2,12)$

11330 FREAD "1PRD"\&" 3" \&" :F8, 1", Power (*)

11331 MASS STORAGE IS ": F8, 1"

11332 PRINTER IS 0

11333 PRINT PAGE

$11340 \mathrm{G}=1$

11350 PRINTER IS 16

11360 File $\$=" C R S T$ " \&" 2 "

11370 FOR $F=1$ TO Nfreqs

11380 CALL Redwrt(File\$, ":F8,1"," ", Result(*), Nfreqs)

11390 FOR $\mathrm{J}=1$ TO 2

11400 FOR $\mathrm{N}=1$ TO 12

11410 PRINTER IS 0

$11420 \operatorname{Wrspt}(J, N)=\operatorname{Result}(F, N+(J-1) * 12)$

11430 NEXT N

11440 NEXT J

11450 ! FOR $I=1$ TO 2

11460 ! Incr $=(I=2) * 5$

11470 ! Inc $=(I=2) * 4$

11480 FOR Mnt=1 TO 8

$11490 \mathrm{Po}(1, \mathrm{Mnt}+\operatorname{Inc})=\operatorname{Power}(\mathrm{F}, 1,1, \mathrm{Mnt}+\mathrm{Incr})$

11500 NEXT Mnt

11510 ! NEXT I

$11520 \operatorname{CALL} \operatorname{Mg}(\mathrm{Po}(*), 1,1, \operatorname{Wrspt}(*)$, "P" , M1 , P1, R1)

11530 CALL $\operatorname{Mg}(\mathrm{Po}(*), 1,2, \operatorname{Wrspt}(*)$, "P",M2, P2, R2)

11540 PRINTER IS 0

11550 PRINT "FREQ \#", F

11552 PRINT

11554 PRINT "DEVICE PLUS ADAPTER ON 6-PORT 1"

11555 PRINT

11560 PRINT "GAMMA MAGNITUDE", "GAMMA PHASE"

11561 PRINT " . . . . . . . . . . " " " . . . . . . . "

11562 PRINT USING 11585;M1,P1

11563 PRINT

11564 PRINT "DEVICE PLUS ADAPTER ON 6-PORT 2"

11565 PRINT

11567 PRINT "GAMMA MAGNITUDE" "GAMMA PHASE"

11568 PRINT " . . . . . . . . . " " " . . . . . .

11570 PRINT USING 11585;M2,P2

11585 IMAGE MD.DDDDD, 12X, MDDD.DDDD

11590 NEXT $F$

11591. NORMAL

11592 PRINT

11593 PRINT

11594 PRINT

11595 PRINTER IS 16

11600 Q=FNAdp (Nfreqs, Repeats, M1, P1 , M2 , P2 , Ca\$)

11610 SUBEXIT

11620 SUBEND

S Gamma( 


\begin{tabular}{|c|c|c|c|c|c|c|}
\hline $\mathrm{Ca} \$$ & 11300 & 11600 & & & & \\
\hline$F$ & 11370 & 11420 & 11490 & 11550 & 11590 & \\
\hline Files & 11360 & 11380 & & & & \\
\hline G & 11340 & & & & & \\
\hline Inc & 11490 & & & & & \\
\hline Incr & 11490 & & & & & \\
\hline $\mathrm{J}$ & 11390 & 11420 & 11420 & 11440 & & \\
\hline M1 & 11520 & 11562 & 11600 & & & \\
\hline M2 & 11530 & 11570 & 11600 & & & \\
\hline Mnt & 11480 & 11490 & 11490 & 11500 & & \\
\hline $\mathrm{N}$ & 11400 & 11420 & 11420 & 11430 & & \\
\hline Nfreqs & 11300 & 11320 & 11320 & 11370 & 11380 & 11600 \\
\hline $\mathrm{P} 1$ & 11520 & 11562 & 11600 & & & \\
\hline P2 & 11530 & 11570 & 11600 & & & \\
\hline Pol & 11320 & 11490 & 11520 & 11530 & & \\
\hline Power ( & 11320 & 11330 & 11490 & & & \\
\hline $\mathrm{Q}$ & 11600 & & & & & \\
\hline RI & 11520 & & & & & \\
\hline R2 & 11530 & & & & & \\
\hline Repeats & 11300 & 11320 & 11600 & & & \\
\hline Result( & 11320 & 11380 & 11420 & & & \\
\hline Wrspt ( & 11320 & 11420 & 11520 & 11530 & & \\
\hline
\end{tabular}




\begin{tabular}{|c|c|c|c|c|c|c|c|}
\hline FNAdp ( & 6470 & 274 & 11600 & & & & \\
\hline FNDerr ( & 4788 & 4563 & 4629 & 4677 & 4776 & & \\
\hline FNDtg\$ & 3129 & 1257 & & & & & \\
\hline FNNumd ( & 3216 & & & & & & \\
\hline FNNumi( & 3183 & 69 & 126 & 153 & 363 & 378 & 819 \\
\hline FNYorn & $\begin{array}{l}3153 \\
1290\end{array}$ & $\begin{array}{r}178 \\
2139\end{array}$ & $\begin{array}{r}219 \\
2916\end{array}$ & $\begin{array}{r}456 \\
3771\end{array}$ & $\begin{array}{r}696 \\
4797\end{array}$ & 795 & 852 \\
\hline S Alinel & 480 & 423 & & & & & \\
\hline S Avg & 3351 & 3969 & & & & & \\
\hline S Cabal & 10440 & & & & & & \\
\hline S Cadal & 10340 & 8440 & 9570 & & & & \\
\hline S Cadd ( & $\begin{array}{l}6183 \\
5877\end{array}$ & $\begin{array}{l}5541 \\
5943\end{array}$ & 5553 & 5559 & 5628 & 5634 & 5835 \\
\hline S Caldat & 1077 & 168 & 272 & & & & \\
\hline S Calibc & 4878 & 3837 & & & & & \\
\hline S Calres & 3612 & 171 & & & & & \\
\hline S Cdial & 10170 & 7480 & 7940 & 8320 & 9500 & & \\
\hline S Cdiv( & $\begin{array}{l}6231 \\
5595\end{array}$ & $\begin{array}{l}5523 \\
5601\end{array}$ & $\begin{array}{l}5526 \\
5619\end{array}$ & $\begin{array}{l}5544 \\
5637\end{array}$ & $\begin{array}{l}5547 \\
5688\end{array}$ & $\begin{array}{l}5562 \\
6105\end{array}$ & 5586 \\
\hline S Cinva( & 10230 & & & & & & \\
\hline S Cmpy( & $\begin{array}{l}6213 \\
5514 \\
5610 \\
6030\end{array}$ & $\begin{array}{l}5331 \\
5529 \\
5631 \\
6099\end{array}$ & $\begin{array}{l}5343 \\
5550 \\
5643 \\
6345\end{array}$ & $\begin{array}{l}5421 \\
5556 \\
5652 \\
6411\end{array}$ & $\begin{array}{l}5496 \\
5574 \\
5655\end{array}$ & $\begin{array}{l}5502 \\
5580 \\
5691\end{array}$ & $\begin{array}{l}5505 \\
5589 \\
5694\end{array}$ \\
\hline S Cmual & $\begin{array}{r}10120 \\
8390\end{array}$ & $\begin{array}{l}7380 \\
9230\end{array}$ & $\begin{array}{l}7650 \\
9280\end{array}$ & $\begin{array}{l}7720 \\
9360\end{array}$ & 7840 & 8100 & 8220 \\
\hline S Cmuada ( & 10730 & & & & & & \\
\hline S Coeff( & 3795 & 3727 & & & & & \\
\hline S Cpral & 10780 & 9140 & & & & & \\
\hline
\end{tabular}




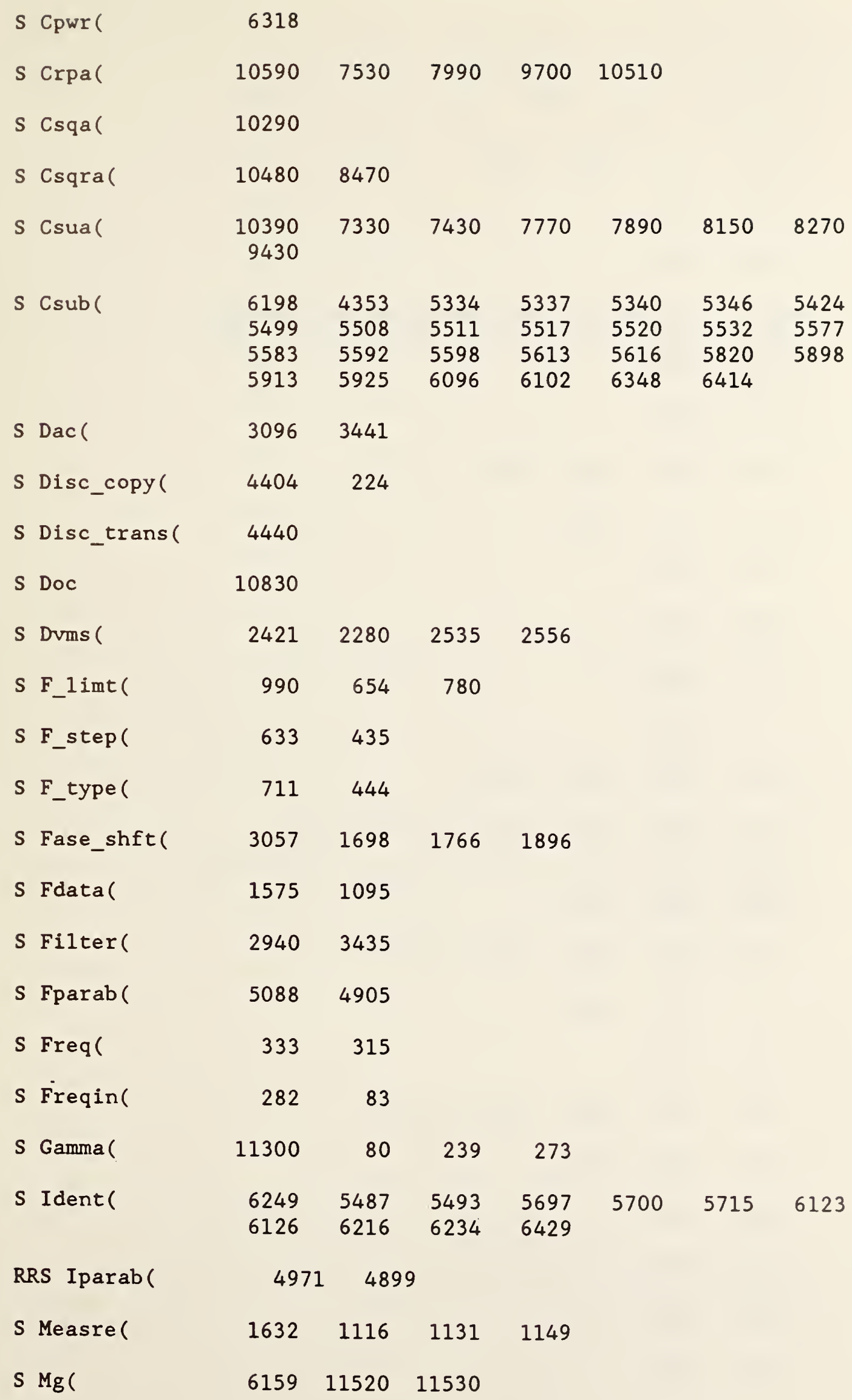




\begin{tabular}{|c|c|c|c|c|c|c|c|c|}
\hline $\mathbf{S}$ & Mpwr ( & 6336 & 6321 & & & & & \\
\hline $\mathbf{S}$ & Msoln( & 6054 & 5040 & 5217 & 6036 & & & \\
\hline$S$ & Mtpt ( & 6372 & & & & & & \\
\hline$S$ & Order ( & 3264 & 777 & 1590 & 1593 & & & \\
\hline$S$ & Path( & 867 & 426 & & & & & \\
\hline$S$ & Pcalc( & 2628 & 1890 & & & & & \\
\hline S & Polar & $\begin{array}{l}6276 \\
5646 \\
6435\end{array}$ & $\begin{array}{l}4863 \\
6108 \\
6438\end{array}$ & $\begin{array}{l}5418 \\
6129\end{array}$ & $\begin{array}{l}5427 \\
6135\end{array}$ & $\begin{array}{l}5436 \\
6171\end{array}$ & $\begin{array}{l}5565 \\
6363\end{array}$ & $\begin{array}{l}5622 \\
6417\end{array}$ \\
\hline$S$ & Pratiol & 5319 & 4950 & & & & & \\
\hline$S$ & Preads ( & 2493 & 1842 & 1854 & 1863 & & & \\
\hline $\mathbf{S}$ & Print & 4020 & 3945 & 3987 & & & & \\
\hline$S$ & Prnt_sdev( & 4194 & 3990 & & & & & \\
\hline s & Prtpwr( & 1356 & 1293 & & & & & \\
\hline$S$ & Pset ( & 3456 & 3438 & & & & & \\
\hline$S$ & Pwrmnt ( & 0 & 1845 & & & & & \\
\hline$S$ & $\operatorname{Rect}($ & 6297 & 4350 & 5685 & 6426 & & & \\
\hline S & Redhed( & 4575 & 3641 & 3646 & 4419 & 4452 & 6640 & \\
\hline S & Redwrt ( & $\begin{array}{l}4689 \\
6820\end{array}$ & $\begin{array}{l}3647 \\
6850\end{array}$ & $\begin{array}{r}3765 \\
11380\end{array}$ & 4422 & 4428 & 4455 & 4464 \\
\hline$S$ & Rfout ( & 3390 & 1722 & 1869 & 1875 & 1881 & & \\
\hline S & Rho2pt( & 4326 & 3891 & 3906 & & & & \\
\hline $\mathbf{S}$ & Rhothu( & 4287 & 3873 & & & & & \\
\hline$S$ & Rtpt & 5985 & 5415 & 5484 & 5490 & 6408 & & \\
\hline $\mathrm{S}$ & Rvgs ( & 2238 & 1728 & 1731 & & & & \\
\hline $\mathrm{s}$ & Stds2( & 5373 & 4947 & & & & & \\
\hline $\mathrm{s}$ & Synth( & 2826 & 3444 & & & & & \\
\hline$S$ & $T_{-}$disc( & 4821 & 3666 & & & & & \\
\hline$S$ & $\operatorname{Tr} 15($ & 5445 & 4944 & & & & & \\
\hline
\end{tabular}




$\begin{array}{llllllll}\text { SWr } & 5709 & 4302 & 4344 & 4924 & 6165 & 6342 & 6381 \\ \text { S Wgl } & 6093 & 4305 & 4347 & 5382 & 5385 & 5388 & 5391 \\ & 5406 & 6138 & 6141 & 6168 & 6357 & 6393 & \\ \text { S Wq } & 6117 & 4158 & & & & & \\ \text { S Wrpl } & 4851 & & & & & & \\ \text { S Wrtbin( } & 4641 & 1280 & & & & & \\ \text { S Wrthed( } & 4494 & 1279 & 3764 & 4425 & 4461 & \end{array}$


NBS.114A (REV. 2-8C)

U.S. DEPT. OF COMM.

BIBLIOGRAPHIC DATA

SHEET (See instructions)

1. PUBLICATION OR
REPORT NO.
1- NBSIR 88-3096

2. Performing Organ. Report No.

3. Publication Date

July 1988

4. TITLE AND SUBTITLE

Measurement of Adapter Loss, Mismatch, and Efficiency Using the

Dual Six-port

5. $\operatorname{AUTHOR}(S)$

feorge J. Counas and Bill C. Yates

6. PERFORMING ORGANIZATION (If joint or other than NBS, see instructions)

NATIONAL BUREAU OF STANDARDS

DEPARTMENT OF COMMERCE

7. Contract/Grant No.

WASHINGTON, D.C. 20234

9. SPONSORING ORGANIZATION NAME AND COMPLETE ADDRESS (Street, City, State, ZIP)

8. Type of Report \& Period Covered

Metrology Engineering Section

Newark Air Force Station, Ohio 43057

10. SUPPLEMENTARY NOTES

Document describes a computer program; SF-185, FIPS Software Summary, is attached.

11. ABSTRACT (A 200-word or less factual summary of most significant information. If document includes a significant bibliography or literature survey, mention it here)

A noise measurement system is being developed for the U.S. Air Force which uses coaxial cryogenic and ambient noise temperature standards to determine the noise temperature of the device under test. When the device under test has a different connector than those on the noise standards, an adapter has to be used. Adapter loss and complex reflection coefficient must be compensated for or noise measurement accuracy is affected. A technique has been developed which uses a dual six-port measurement system to determine the mismatch, loss, and ultimately the efficiency of the adapter used. This enables correction of measurement results and allows measurements to be made with an adapter with no degradation of accuracy.

This report describes this method of evaluating adapters and provides instructions for its use.

12. KEY WORDS (Six to twelve entries; alphabetical order; copitalize only proper names: and separate key words by semicolons) adapter parameters, dual six-port measurement system, efficiency, loss, measurement technique, mismatch

13. AVAILABILITY

X] Untimited

For Official Distribution. Do Not Release to NTIS

Order From Superintendent of Documents, U.S. Government Printing Office, Washington, D.C. 20402.

14. NO. OF

PRINTED PAGES

208

15. Price

X] Order From National Technical Information Service (NTIS). Soringfield, VA. 2216I

* U. S. GOVERNMENT PRINTING OFFICE: 1988 - $576195 / 85194$ 

